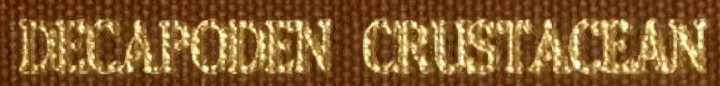

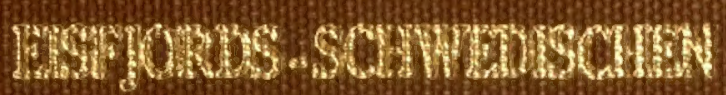

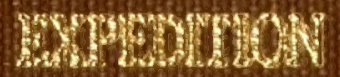

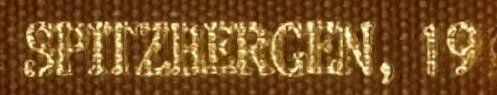

101 



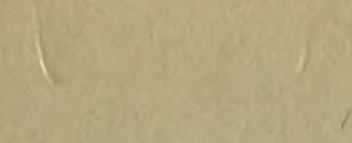

$$
\text { CID F B B }
$$





KUNGL. SVENSKA VETENSKAPSAKADEMIENS HANDLINGAR. Band 54. N:0 7.

\section{ZOOLOGISCHE ERGEBNISSE}

DER

SCHWEDISCHEN EXPEDITION NACH SPITZBERGEN 1908

UNTER LEITUNG VON PROF. G. DE GEER

REDIGIERT VON N. voN HOFSTEN UND S. BOCK

TEIL II.

7

\section{DIE DECAPODEN CRUSTAACEEN DES EISFJORDS}

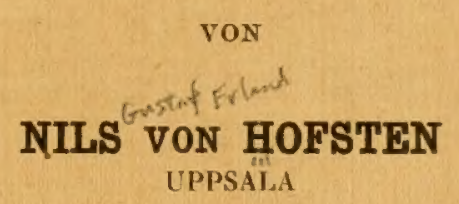

MIT 2 TAFELN ( 4 KARTEN) UND 13 TEXTFIGUREN (DAVON 12 KARTEN)

MITGETEILT AM 7. JUNI 1916 DURCH HJ. THÉEL UND E. LÖNNBERG
1916
STOCKHOLM
ALMQVIST \& WIKSELLIS BOKTRYCKERI-A.-B.
BERLIN
R. FRIEDLÄNDER \& SOHN
11 CARLSTRASSE
LONDON
WILLIAM WESLEY \& SON
28 ESSEX STREET. STRAND
PARIS
LIBRAIRIE C. KLINCKSIECK.
11 RUE DE LILLE 

KUNGL. SVENSKA VETENSKAPSAKADEMIENS HANDLINGAR. Band 54. N:0 7.

\section{ZOOLOGISCHE ERGEBNISSE \\ DER}

SCHWEDISCHEN EXPEDITION NACH SPITZBERGEN 1908

UNTER LEITUNG VON PROF. G. DE GEER

TEIL II.

7.

DIE DECAPODEN CRUSTACEEN DES EISFJORDS

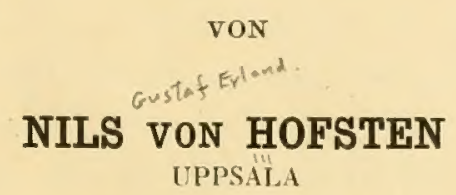

MIT 2 TAFELN (4 KARTEN) UND 13 TEXTFIGUREN (DAVON 12 KARTEN)

MITGETEILT AM 7. JUNI 1916 DURCH HJ. THÉEL UND E. LÖNNBERG

STOCKHOLM

ALMOVIST \& WIKSELLS BOKTRYCKERI-A.-B. 


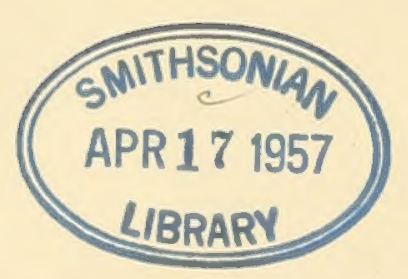




\section{Vorwort.}

Die im Speziellen 'Teil dieser Arbeit behandelten Arten wurden von der schwedischen Expedition nach Spitzbergen 1908 im Eisfjord gesammelt. Im Allgemeinen Teil werden ausserdem Decapoden von andern arktischen Gegenden besprochen.

Die Arbeit hat einen rein tiergeographisch-ökologisehen Zweck; über die allgemeinen Gesichtspunkte und Prinzipien, denen ich bei der Untersuchung gefolgt bin, kann ich mich hier nicht äussern, sondern verweise auf meine Arbeit iiber die Echinodermen des Eisfjords. ${ }^{1}$ Auch in Bezug auf die praktische Darstellungsweise (Fundortslisten, Schemata der Vertikalverbreitung, Literaturangaben, Verbreitungskarten, tiergeographische Nomenklatur usw.) muss auf die erwähnte Arbeit hingewiesen werden.

Von den älteren schwedischen Expeditionen ist ein nicht unbeträchtliches Material von Decapoden im Eisfjord gesammelt worden. Durch freundliches Entgegenkommen von Dr. A. Molander, der die im Reichsmuseum zu Stockholm aufbewahrten arktischen und nordischen Decapoden bestimmt hat, bin ich in der Lage, diese bisher nicht veröffentlichten Funde im Eisfjord mitzuteilen.

Das Manuskript zu dieser Arbeit war schon im Frühling 1913 fertiggestellt, ist aber später umgearbeitet worden; ich habe dabei versucht, wenigstens die wichtigere neuere Literatur zu beriicksichtigen. Nach der Einlieferung des Manuskriptes im Februar 1915 erschien eine wichtige Arbeit von Dons (1915); Hinweise auf die wichtigsten Angaben wurden nachträglich eingeführt.

1 Zool. Erg. d. schwed. Exped. n. Spitzb. 1908, T. II, N:o 2, 1915 (K. Sץ. Vet. Akad. Handl., Bd. 54). 


\section{Spezieller Teil.}

\section{Spirontocaris ${ }^{1}$ spinus (Sow:).}

Syn. S. sowerbyi (LEACH).

\section{Verbreitung im Eisfjord.}

Verzeichnis der Fundorte (s. Karte 3):

\begin{tabular}{|c|c|c|c|c|c|c|c|}
\hline $\begin{array}{l}\text { Nr. } \\
\text { der } \\
\text { Stat. }\end{array}$ & Ort und Datum & 'Tiefe & $\begin{array}{l}\text { Wasser- } \\
\text { temperatur }\end{array}$ & $\begin{array}{c}\text { Salz- } \\
\text { gehalt } \\
\%\end{array}$ & Bodenbeschaffen heit & Gerät & $\begin{array}{l}\text { Anzahl und } \\
\text { Grösse }^{2}\end{array}$ \\
\hline 42 & $\begin{array}{l}\text { Svonsksundstiofe } \\
\text { (Eingang in den } \\
\text { Fjorl)......21.7 }\end{array}$ & $406-395 \mathrm{~m}$ & $382 \mathrm{~m}:+2,61^{\circ}$ & 34,90 & Loser Schlamm & Trawl & $2 \mathrm{Ex}, 39 \mathrm{~mm}$ \\
\hline 43 & $\begin{array}{l}\text { Svensksundstiefo } \\
\text { (Eingang in den } \\
\text { Fjord) . . . 25.7 }\end{array}$ & $228-257 \mathrm{~m}$ & $228 \mathrm{m:}+2,74^{\circ}$ & 34,90 & ») & Ottertrawl & $1 \mathrm{Ex}, 64 \mathrm{~mm}$ \\
\hline+ & $\begin{array}{l}\text { Svensksundstiefo } \\
\text { (Eingang in den } \\
\text { Fjord) . . . 16.7 }\end{array}$ & $277-313 \mathrm{~m}$ & {$\left[\right.$ Etwa $\left.+2,5^{\circ}\right]$ s } & - & Schlamm & 》 & $1 \mathrm{Ex}, 56 \mathrm{~mm}$ \\
\hline 13 & $\begin{array}{l}\text { Eingang in dio Safo } \\
\text { Bay } . . .16 .7\end{array}$ & $125-150 \mathrm{~m}$ & $\left|\begin{array}{l}125 \mathrm{~m}:+0,87^{\circ} \\
144 \mathrm{~m}:+1,23^{\circ} \mathrm{i}\end{array}\right|$ & $\left|\begin{array}{c}34,52 u \\
34,54\end{array}\right|$ & $\begin{array}{l}\text { Schlamno mit Schalen; } \\
\text { Balanus porcatus-Ge- } \\
\text { meinsch. }\end{array}$ & Trawl & $\begin{array}{l}24 \mathrm{Ex}, 37- \\
57 \mathrm{~mm}(3 \text { \% } \\
\text { ovig. }\end{array}$ \\
\hline 6 & Safe Bry . . . 15.7 & $40 \mathrm{~m}$ & - & - & Schlamm, etwas Stein & kl. Dredge & $1 \mathrm{Ex} ., 40 \mathrm{~mm}$ \\
\hline 41 & Fjordstamm . . . 24.7 & $234-254 \mathrm{~m}$ & $251 \mathrm{~m}:+2,56^{\circ}$ & 34,96 & Loser Schlamm & Trawl & $\underset{\mathrm{mm}}{2 \mathrm{Ex}, 32,64}$ \\
\hline 21 & $\begin{array}{l}\text { Eingang in dio Tund- } \\
\text { r̊ Bay. . . . 20.7 }\end{array}$ & $71-68 \mathrm{~m}$ & $-0,93^{\circ}$ & 34,29 & $\begin{array}{l}\text { Loser Schlamm, stellen- } \\
\text { weise Stein }\end{array}$ & , & I Ex., $50 \mathrm{~mm}$ \\
\hline
\end{tabular}

1 Mehrero Autoren gebrauchen heuto noch für diese und dio folgenden Arten den Gattungsnamen IIippolyte.

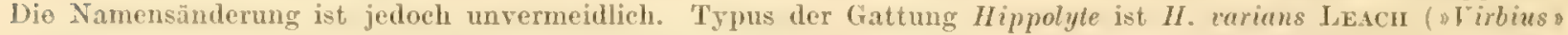
varians aut.). Dio von den neueren russischen Autoren (BRAsminow, Brives) durchgeführto Zerlegung der Gattung spirontoctris liabe ich nicht aufgenommen, weil die von ihnen untersehiedenen Gattungen wohl gegenwärtig besser als Subgenera aufzufassen sind.

${ }^{2}$ Die Masso bedeuten die Länge des roll ausgestreckten Tieres, von der Spitze des Rostrums bis zum Ende des Telsons gemessen.

3 Die in cekige Klammern gesetzten Temperaturangaben sind nicht durch direkte Beobachtung gleichzeitig mit der Dredgung gewonnen, sondern uach den Verhältnissen an benachbarten Stellen oder bisweilen nach dem allgemeinen hydrographischen Zustand des Fjords berechnet. Die römischen Ziffern renweisen auf a $\mathrm{m} \mathrm{E} n \mathrm{~d} e$ der $A \mathrm{rb}$ it zusammengestellte Anmerkungen, die über die Art der Berechnung Aufschluss geben. 


\begin{tabular}{|c|c|c|c|c|c|c|c|c|}
\hline $\begin{array}{l}\text { Nr. } \\
\text { der } \\
\text { Stat. }\end{array}$ & Ort und & Datum & Tiefo & $\begin{array}{l}\text { Wasser- } \\
\text { temperatur }\end{array}$ & $\begin{array}{l}\text { Salz: } \\
\text { gehalt } \\
\%\end{array}$ & Bodenbeschaffenheit & Gerät & $\begin{array}{l}\text { Anzahl und } \\
\text { Grösso }\end{array}$ \\
\hline 94 & Fjordstamm & $\ldots 21.8$ & $147-141 \mathrm{~m}$ & $140 \mathrm{~m}:-0,62^{\circ}$ & 34,49 & $\begin{array}{l}\text { Loser Schlamm mit klei- } \\
\text { nen Steinen }\end{array}$ & Trawl & $\begin{array}{l}4 \text { Ex., } 47- \\
63 \mathrm{~mm}(2 \text { \% } \\
\text { ovig. })\end{array}$ \\
\hline 98 & Nordarm .. & $\ldots 27.8$ & $130-116 \mathrm{~m}$ & $115 \mathrm{~m}:-0,82^{\circ}$ & 34,40 & Loser Schlamm & $»$ & $1 \mathrm{Ex}, 44 \mathrm{~mm}$ \\
\hline 102 & $\begin{array}{l}\text { Nordarm. Ei } \\
\text { die Yoldia }\end{array}$ & $\begin{array}{l}\text { ingang in } \\
\text { Bay } .14 .8\end{array}$ & $70-93 \mathrm{~m}$ & $85 \mathrm{~m}:+0,68^{\circ}$ & 34,25 & $\begin{array}{l}\text { Zäher und fester Schlamm } \\
\text { mit vielen Steinen }\end{array}$ & $"$ & $\underset{\mathrm{mm}}{2 \mathrm{Ex}_{0}, 40,42}$ \\
\hline 93 & Ekman Bay & $\ldots 20.8$ & $44-55 \mathrm{~m}$ & $+1,72^{\circ}$ & - & $\begin{array}{l}\text { Sehr zäher, stark loser } \\
\text { Schlamm. Etwas Stein }\end{array}$ & $»$ & 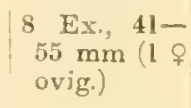 \\
\hline 116 & $\begin{array}{l}\text { Nordarm, } \\
\text { Eingang } \\
\text { Dickson } \mathrm{B}\end{array}$ & $\begin{array}{l}\text { Vor dem } \\
\text { in dio } \\
\text { ay . . } 258\end{array}$ & $57-60 \mathrm{~m}$ & $+1,2^{\circ}$ & - & Kies und Stein & K1. Dredge & $\mid \begin{array}{l}1 \mathrm{Ex}, \\
\text { ovig.), }, 53 \mathrm{~mm}\end{array}$ \\
\hline 79 & Billen Bay & $\ldots 13.8$ & $32-40 \mathrm{~m}$ & {$\left[+1,5\right.$ bis $\left.+2^{\circ}\right] \times v$} & - & $\begin{array}{l}\text { Steine mit strauchför- } \\
\text { migem Lithothamnion }\end{array}$ & $"$ & $\underset{\mathrm{mm}}{2 \mathrm{Ex}, 43,45}$ \\
\hline 80 & $\begin{array}{c}\text { Eingang in } d \\
\text { Bay ... }\end{array}$ & dio Billen & $69-64 \mathrm{~m}$ & $69 \mathrm{~m}:+1,5^{\circ}$ & - & $\begin{array}{r}\text { Loser Schlamm (mit ein } \\
\text { wenig Stein und Kies) }\end{array}$ & m. & $2 \underset{\mathrm{mm}}{2 \mathrm{Ex},}, 37,45$ \\
\hline 82 & Billen Bay. & $\cdots .158$ & $65 \mathrm{~m}$ & $-0,7^{\circ}$ & 一 & $\begin{array}{l}\text { Teils loser Schlamm, teils' } \\
\text { fester Schlamm mit } \\
\text { Steinen und Kies }\end{array}$ & " & $\underset{\mathrm{mm}}{2 \mathrm{Ex} .}, 38,39$ \\
\hline 101 & ?. & $\ldots .14 .8$ & $150-140 \mathrm{~m}$ & $140 \mathrm{~m}:-1,67^{\circ}$ & 34,43 & $\begin{array}{c}\text { Loser Schlamm mit Stei- } \\
\text { nen }\end{array}$ & Trawl. & $\begin{array}{l}2 \mathrm{Ex}, 34,37 \\
\mathrm{~mm}\end{array}$ \\
\hline 49 & Sassen Bay, & Bank - 31.7 & $\begin{array}{c}24-19 \text { und } \\
19-28 \mathrm{~m}\end{array}$ & {$\left[+2 \text { bis }+3^{\circ}\right]^{\mathrm{xIx}} \mid$} & - & $\begin{array}{l}\text { Stein, Kies und Schalen } \\
\text { mit Lithothamnion }\end{array}$ & D & $\begin{array}{l}3 \text { Ex., } 35,47 \\
53 \mathrm{~mm}(1 \text { o } \\
\text { ovig.) }\end{array}$ \\
\hline 47 & $\begin{array}{l}\text { Eingang in di } \\
\text { Bay ... }\end{array}$ & 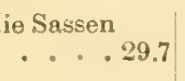 & $97-120 \mathrm{~m}$ & $\begin{array}{c}\text { [etwa } 0^{\circ} \text { ] XIV } \\
\left(82 \mathrm{m:}+1,71^{\circ}\right)\end{array}$ & - & Loser Schlamm & Ottertrawl & $\begin{array}{l}4 \mathrm{Ex}, 52-61 \\
\mathrm{~mm}\end{array}$ \\
\hline 48 & Ostarm .. &. .31 .7 & $199-226 \mathrm{~m}$ & $210 \mathrm{~m}:+1,27^{\circ} \mid$ & 34,72 & $"$ & Trawl & 1 Ex., $63 \mathrm{~mm}$ \\
\hline 44 & $\begin{array}{l}\text { Eingang in } \\
\text { vent Bay }\end{array}$ & die Ad. & $150-110 \mathrm{~m}$ & $128 \mathrm{~m}:+0,01^{\circ}$ & 34,54 & Loser Schlamm mit Kies & » & $\begin{array}{l}5 \text { Ex., } 41- \\
62 \mathrm{~mm}(1 \text { o } \\
\text { ovig.) }\end{array}$ \\
\hline 73 & Advent Bay & $\ldots 11.8$ & $35-30 \mathrm{~m}$ & \begin{tabular}{c|}
{$[+2$ bis } \\
$\left.+2,7^{\circ}\right] \times \times 1 I$
\end{tabular} & - & $\begin{array}{l}\text { Balanus porcatus-Ge- } \\
\text { meinsch. Kies und Stein }\end{array}$ & Ii. Dredge & $1 \mathrm{Ex} ., 56 \mathrm{~mm}$ \\
\hline 95 & Fjordstamm & $\ldots 21.8$ & $188-181 \mathrm{~m}$ & $\left|\begin{array}{c}\left.10 \mathrm{bis}+0,5^{\circ}\right] \text { vir } \\
\left(163 \mathrm{~m}:-0,11^{\circ}\right)\end{array}\right|$ & $\left(\begin{array}{c}163 \mathrm{~m}: \\
34,47)\end{array}\right.$ & Schlamm mit Steinen & Trawl & $\underset{\mathrm{mm}}{2 \text { Ex, } 33,54}$ \\
\hline 69 & Coles Bay . & . . 8.8 & $71 \mathrm{~m}$ & $\left|\begin{array}{c}{[+1,5 \text { bis }} \\
\left.+2^{\circ}\right]^{\operatorname{Jxiv}}\end{array}\right|$ & - & $\begin{array}{c}\text { Kies, Stein und Schalen. } \\
\text { Etwas Lithothamnion }\end{array}$ & Kil. Dredge & ${ }_{\mathrm{mm}}^{2 \mathrm{Ex}, 35,37}$ \\
\hline 97 & Fjordstamm & $\cdots 23.8$ & $243-230 \mathrm{~m}$ & {$\left[+2 \mathrm{bis}+2,5^{\circ}\right] \mathrm{vIr}$} & - & Loser Schlamm & $\begin{array}{c}\text { Trawl } \\
\text { (Netz unklar) }\end{array}$ & $\mid \begin{array}{l}1 \text { Ex., }(q \\
\text { ovig.), } 52 \mathrm{~mm}\end{array}$ \\
\hline 96 & Fjordstamm & $\cdot .22 .8$ & $\begin{array}{l}230 \text { - etiva } \\
200 \mathrm{~m}\end{array}$ & $208 \mathrm{m:}+2,56^{\circ}$ & 34,76 & $\begin{array}{l}\text { Schlamm mit etwas Stein } \\
\text { und Kies }\end{array}$ & Trawl & $\begin{array}{l}11 \mathrm{Ex}, 17- \\
58 \mathrm{~mm}\end{array}$ \\
\hline 103 & $\begin{array}{c}\text { Green Bay na } \\
\text { Eingang . }\end{array}$ & ahe beim & $130 \mathrm{~m}$ & $+0,58^{\circ}$ & - & $\begin{array}{l}\text { Loser Schlamm. Einige } \\
\text { Steine und Balanus por- } \\
\text { catus }\end{array}$ & " & $\underset{\mathrm{mm}}{\mathrm{C} \cdot \mathrm{Ex}, 30-57}$ \\
\hline 130 & Groen Bay & . . 30.8 & $40-45 \mathrm{~m}$ & - & - & Schlamm mit Algenresten & K1. Dredge & $\frac{10 \mathrm{Ex}, 27-}{60 \mathrm{~mm}}$ \\
\hline 59 & ” & $\ldots .3 .8$ & Etwa $40 \mathrm{~m}$ & - & - & $\begin{array}{l}\text { Sehr loser Schlamm mit } \\
\text { Teilen von Landpflan- } \\
\text { zen usw. }\end{array}$ & s & 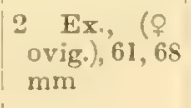 \\
\hline 60 & " & $\cdots \cdot$ & $33 \mathrm{~m}$ & 一 & - & $\begin{array}{l}\text { Kies, Stoin und Schalen } \\
\text { mit Lithothamnion-Kru- } \\
\text { sten; zahlreiche Balanus } \\
\text { porcatus-Kolonien }\end{array}$ & , & ${ }_{45 \mathrm{~mm}}^{6 \text { Ex, } 29-}$ \\
\hline 61 & $"$ & $\cdots 4.8$ & $46-35 \mathrm{~m}$ & - & - & $\begin{array}{l}\text { Kies und Stein. Balanus } \\
\text { porcatus-Gemeinsch. }\end{array}$ & " & $\begin{array}{l}6 \mathrm{Ex} ., 38- \\
56 \mathrm{~mm}(2 \text { \% } \\
\text { ovig.) }\end{array}$ \\
\hline
\end{tabular}

[Ausser in Eisford fanden wir S. spinus im Hornsund (Goës' Bay, 10.7, 25 m, Schlamm mit hies, 1 Ex.)] 


$$
\text { Frülere Funde im Lis f jord. }
$$

Sehwed. Lixped. 1858: Eisfjord ohne nähero Angaben; 1864: Safe 13ay; 1873: Green Bay, $80 \mathrm{~m}$ (Riksmuscum, Stockholm, nach A. Morander). Norw. Nordmeerexped. 1878: Adrent Bay, $110 \mathrm{~m}$, Temp. +0, $7^{\circ}$, Schlamm (S.ARs 1886). Schwed. Exped. 1890: Eingang, $180 \mathrm{~m}$ (Kirsckowströs 1892). Olga-Exped. 1898: Adrent Bay (DoF. LEIN 1900). Schwed. Exped. 1899: Green Bay, $110 \mathrm{~m}$; 1900: Coles Bay, $100 \mathrm{~m}$, felsig: $50 \mathrm{~m}$, Stein: 50 bis $100 \mathrm{~m}$, Stein (Ondx 1901). - Nach Collett (1905) hätte die Michael Sars-Expedition 1901 in der Groen Bay zahlreiche Lixemplare der verwandten borealen Art spirontocaris securifrons (Nors.) $=$ S. lilljeborgi (DAxielss.) gefunden (im Magen von Icelus bicornis). Da dieser Fund nur ganz beilüufig erwähnt wird und der Autor, so viel ich weiss, sich nio mit Crustaceen beschäftigt hat, kann man mit Sicherheit annehmen, dass nicht die südliche, sonst nie im Spitzbergengebiet gefundene S. lilljeborgi, sondern dio in der Green Bay allgemeino S. spinus vorgelegen hat.

Wir fanden Spirontocaris spinus an 30 Fundorten, also ungefähr ebenso oft wie S. turgida (31 Stationen). Da die bathymetrische Verbreitung weniger beschränkt ist als bei der letzteren, dürfte die Art als die verhältnismässig seltenste Macrurenspecies des Eisfjords bezeichnet werden; auch scheint sie oft in geringerer Individuenzahl als die meisten übrigen Macruren aufzutreten (insgesamt fanden wir 116 Exemplare). Ein seltenes 'I'ier ist sie ja aber natiirlich keineswegs.

In bezug auf die $B$ o d e $n$ be s e h a f f e n h e i t ist die Art nicht sehr wählerisch; an losem, ungemischtem Schlammgrund ist sie jedoch nicht häufig (wir fanden sie zwar an 8 solchen Stationen, aber in zusammen nur 13 Exemplaren). Verhältnismässig am häufigsten ist sie in den Balanus porcatus-Kolonien.

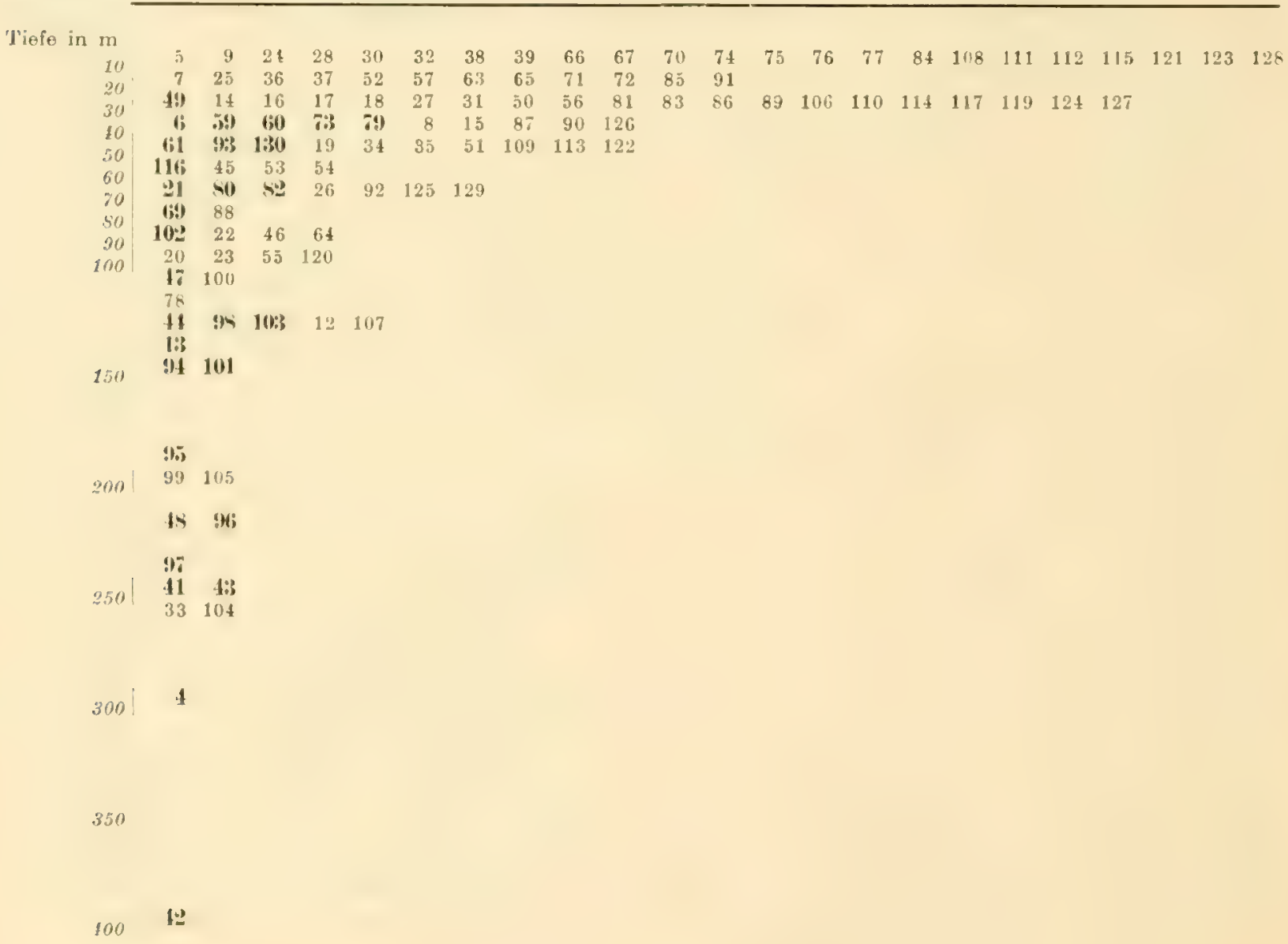


Die vertikale Verbreitung erstreckt sich von etwa 25 bis $400 \mathrm{~m}$. Wie die vorstehende Úbersicht zeigt, ist die Art selten oberhalb von etwa $35 \mathrm{~m}$; schon von $40 \mathrm{~m}$ an ist sie ungefähr gleich häufig bis in die grösste Tiefe des Fjords. Dass sie in der Tat auch dort verhältnismässig häufig ist, ergibt sich daraus, dass sie an 7 der 9 unterhalb von $200 \mathrm{~m}$ gelegenen Stationen erbeutet wurde. Von $230 \mathrm{~m}$ an fanden wir an jedem Fundort nur 1 oder 2 Exemplare. Da die Art in allen Tiefen ort vereinzelt auftritt, beweist dies nicht viel; möglicherweise kann man den Schluss ziehen, dass sie in den grösseren Tiefen an Häufigkeit abnimmt. - Unsere Funde lehren jedenfalls, dass die Art bis in $400 \mathrm{~m}$ Tiefe hinabsteigt; die grösste mit Sicherheit bekannte Tiefe war früher $330 \mathrm{~m}$ (BATE 1888; nach HANSEN [1909] $315 \mathrm{~m}$ ).

Die obere T e m pera t u r g r en ze (Sommertemperatur; also die höchste Temperatur, welcher die Art überhaupt ausgesetzt ist) liegt im Eisfjord bei $+2,7 t^{\circ}$, die untere bei $-1,67^{\circ}$. Unsere Beobachtungen zeigen zunächst (s. die nachstehende Übersicht), dass $S$. spinus in Wasser von niedrig positiver Temperatur $\left(0\right.$ bis $\left.+3^{\circ}\right)$ sehr häufig ist (an 20 von 47 Stationen gefunden; hier eine verhältnismässig grosse Individuenzahl und die kräftigsten Exemplare). In Wasser von negativer Temperatur fanden wir die Art weniger häufig (5 Stationen, 10 Exemplare), obgleich fast alle solche Stationen in Tiefen liegen, welche das Tier sonst bewohnt. Da die Art in verschiedenen hocharktischen Gegenden vorkommt, muss ich trotzdem annehmen, dass die Seltenheit an den von uns untersuchten kalten Steilen des Eisfjords nicht durch die Temperaturverhältnisse bedingt ist. Trotz des verhältnismässig ubiquistischen Charakters dieser Art dürfte wohl die Bodenbeschaffenheit dafür verantwortlich sein; die meisten kalten Stellen, an welchen das Tier fehlte, hatten losen Schlammgrund oder befanden sich unweit von Gletschern, wo wir stets ein spärliches Tierleben fanden.

\begin{tabular}{|c|c|c|c|c|c|c|c|c|c|c|c|c|c|c|c|c|c|c|c|c|c|}
\hline$+4,5-+5,6^{\circ}$ & 24 & 28 & 32 & 38 & 39 & 66 & 67 & 70 & 71 & 75 & 76 & 77 & 84 & & & & & & & & \\
\hline$+3-+40$ & 5 & 7 & 9 & 50 & 52 & 56 & 57 & 72 & 85 & 89 & 91 & 108 & 111 & 112 & 115 & 121 & 123 & 127 & 128 & & \\
\hline-+30 & 4 & 41 & 42 & 43 & 49 & 73 & H; & 97 & 18 & 19 & 30 & 33 & 36 & 51 & 71 & 92 & 106 & 110 & 117 & 124 & 126 \\
\hline$-+1,6^{\circ}$ & 13 & 48 & 69 & 79 & 80 & 93 & 116 & 26 & 37 & 45 & 81 & 83 & 86 & 87 & 104 & 109 & & & & & \\
\hline $0-+0,3^{\circ}$ & $H$ & 47 & 95 & 102 & 108 & 12 & 14 & 16 & 17 & 99 & & & & & & & & & & & \\
\hline$-0,9-+0,1^{\circ}$ & 21 & S: & 94 & 98 & 15 & 20 & 22 & 23 & 34 & 35 & 53 & 78 & 107 & 113 & 114 & 122 & & & & & \\
\hline$-1,5--1,3^{\circ}$ & 101 & 54 & 55 & 88 & 100 & 105 & 120 & 125 & & & & & & & & & & & & & \\
\hline
\end{tabular}

Das Fehlen in der obersten, warmen Zone des Fjords beweist nicht, dass die Art Wasser von mehr als $+2,5$ bis $3^{\circ}$ Sommertemperatur meidet; sie lebt nämlich auch in hocharktischen Gegenden bloss in mehr als 20 oder $25 \mathrm{~m}$ Tiefe (s. besonders OHuIN 1895, 1901, Doflein 1900, Ortmann 1901, Birula 1907, Stephensen 1912). Andrerseits folgt daraus natürlich nicht, dass sie wärmeres Wasser erträgt. Bei den ungenügenden Kenntnissen der Lebensweise in den boreoarktischen Gebieten muss die Frage nach der oberen Temperaturgrenze für das regelmässige Vorkommen in der arktischen und boreoarktischen Region (bei etwa $+3^{\circ}$ oder etwas höher?) offen gelassen werden. Dass die Art vereinzelt im borealen Gebiet auftreten kann, beweist in dieser Frage nichts.

Fortpflanzung, Entwi c klung. Eiertragende Weibchen (unter diesem Ausdruck bezeichne ich stets Weibchen mit unter dem Hinterleib abgesetzten Eiern) wurden 9mal erbeutet (St. 13, 94, 93, 116, 49, 44, 97, 59, 61). Thre Grösse wechselt zwischen 44 und $68 \mathrm{~mm}$. Nach Birula (1907) sind die Grössensehwankungen der eiertra- 
genden Weibchen kleiner (4s - $61 . .5 \mathrm{~mm}$ ), nach Dofleix (1900) dagegen grösser (25-60 mmin) als an moserem Material. Die weibliche (ieschlechtsreife dürfte jedoch kaum früher als bei einer Körperlänge ron etwa $40 \mathrm{~mm}$ eintreten; die Angabe DofLEIN's ist wahrscheinlich dadurch zu erklären, dass er S. spinus und turgida nicht voneinander trennt. - Das grösste von uns beobachtete, $68 \mathrm{~mm}$ lange Lxemplar, dürfte als ungewöhnlich gross bezeichnet werden; ron den früheren Auroren gibt OHLIN $(1895,1901)$ eine Maximallänge von $62 \mathrm{bzw}$. $66 \mathrm{~mm}$, Biruld eine Maximallänge von $67 \mathrm{~mm}$ an.

Die Eier befinden sich ausnahmslos in den frühesten Entwicklungsstadien sowohl bei den Mitte Juli wie bei den Ende August gefangenen Exemplaren; dasselbe gilt von den von der Kolthoff-Expedition in der Coles Bay (16,-22.6. 1900) und im W. von Spitzbergen (2.7) gefundenen Exemplaren. An den letzteren und an den von uns Mitte Tuli gefangenen Tieren sind die Eier, wie es scheint, ganz unentwickelt; an den am Ende des Sommers gefundenen ist die Entwicklung teilweise (besonders St. 97, 23.8.) cin wenig weiter vorgeschritten als bei den übrigen. Nan kann hieraus schliessen, dass im Ëisford die Eiablegung ganz oder teilweise im Frïling oder Sommer, die Entwicklung der Embryonen aber fast nur im Herbst oder Winter geschieht. Im boreoarktischen 'Teil des Nordmeer's fundet die Entwieklung ebenfalls im Winter statt (Nordiand) [1905] fand Embryonen mit Augen 3.-24.4), doch liegen nicht dieselben Verhältnisse wie im Eisfjord vor, denn die Absetzung der Eier geschieht erst in der kalten Jahreszeit (DONS 1915).

\section{Allgemeine Terbreitung.}

(Fig. 1.)

$$
\text { Spitzborgengobiot: }
$$

Westspitzbergen: Südkap, W. ron dem Hornsmd, Belsund, Eisfjord, Kingsbay (Dortern 1900, OnfiN 1901, Bmura 1907; ferner Kroyer 1842: allgemein bei spitzbergen). Nordwest p itzboxgon und westlichster Teil der Nordküste (Dorlein 1900, OnLiv 1901). Ostspitz borgon: (iiles Land, K. Karls Land, Storfjord sowio O. und S. daron (HoEk 1882, Doflein 1900, STEBbing 1900, OHLIn 1901). Hopen Eiland (Dons 1915). He r o n E I and (Kunckowströn 1892).

Über dio Verbreitung im Spitzbergengebiet bemerkt DofLeIN (1900), dass die Art von den deutschen Expeditionen 1898 ovorwiegend in Ostspitzbergen. und "nur in kaltem Wasser... bei warmem Oberflächenwasser nur in der Tiefo" gefunden wurdo (vgl. auch seino Verbreitungskarte, S. 358); sio wïre daher, wio dio Spirontocaris. Arten iberhaupt, im Sommer 1898 "ganz und gar auf das in jenem Jahre bedeutend eingeschränkte Kaltwasser. gobiet in ihrer Verbroitung begrenzt gewesen. Auch BIRULA (1907) behauptet, dass sie vorzugsweise in Ostspitzbergen erbentet worden sei. Diese Schlussfolgerungen sind zwoifellos unrichtig. Wio besonders unsore Funde zeigen, ist S. spinus hänfig in Westspitzbergen, sicher ganz ebenso allgemein wie im Osten. Im Sommer 1898 hatto nun das Golfstromswasser eine auffallend weite Verbreitung und hohe Temperatur. Im Eisfjord waren jedoch die 'T'emperaturverhältnisso (wio schon die wenigen von der Helgoland-Expedition gemachten Oberflächenmessungen andenten) nicht wesentlich verschieden ron den 1908 herrschenden; S. spinus gedeilit, wio ich oben gezeigt habo, sohr gut in Wasser von niedrig positiver 'Temperatur und muss folglich s $t$ o $t$ s günstigo Lobensbedingungen im Eisfjord - und an der ganzen Westseite von Spitzbergen - finden. Dass dies in der 'l'at auch i. J. 1898 dor l'all war, wird dnrch dio dann gomachten Fundo in Westspitzbergen, besonders dureh den Fund der Olga-Expedition in der Advent Bay, angedeutet, der von DofLeIN zwar im Text erwähnt, aber nielst auf das Verbreitungskärtehen eingetragen wordon ist.

Übrigo arktischo und borooarktischo Rogion:

Barentsmeer, Westküste ron Novaja Semlja (Hokk 1882, Dovs 1915). Südküsto von Novaja Semlja, Ǩarische Pforte (STAPpers 1911). Weisses Heer (Lokalangaben nur im Eingang), Murmanküste (JARzYsiskY 1885, PFEFFer 1890, BIRUla 1897, 1899, DOFlein 1900, DerJugin 1906). Ostfinnmarken (DaNierssex 1861, Dons 
1915; forner M. SARs 1858: "Finmmarken»). Kalto F'jorde in Westfinmmarken und den Lofoten (s. unten). - Jau Mayen (KOELBEL 1886). Ostküste von Island (HANSEN 1908; ferner APPELLöF 1906: vboreoarktische liegion Islands "). Nordostgrönland, Südostgrönland, Westgrönland, Nordwestgrönland (s. STEpHensex 1913, ferner K Rör ER 1838, WALKER 1860, NoRMAN 1877, MIERS 1880, HANSEN 1887, 1908, 1909, VANIÖFFEN 1897, OIILIN 1895, 1901, Ortmann 1901, Stepinensen 1912, 1912 a, 1916$){ }^{1}$ - Arktisches Nordamerika: Ellesmero Land (Mifers 1877 a); Jones Sund (SARs 1909); Melville-Hallinsel (Iglootik Island) (Ross 1835). - Nordostküste von Labrador, Golf von St. Lawrence, Ostküste von Nordamerika bis K. Cod (PACKARD 1863, 1866, StrMpson 1871, Verrilu 1873, 1874, Verrill \& Rathbon 1880, Whiteaves 1874, 1901, Smiti \& HARger 1874, SMiti 1879, 1884, 1884 a, Bate 1888, Howe 1901, Ratibun 1905). - Pazifisches Gebiet: Nordküste von Alaska, Beringsstrasse, Beringsmeer, Aleuten; auch (1 Fundort) Südostalaska (STIMPSON 1860, Richrers 1884, besonders RaTuBUN 1904); Ostküsto ron Kamschatka, Ochotskisches Meer (Brasinikow 1907). [Nlordocn's S. spinus von der Nordküsto Alaskas soll dagegen nach RATIBUN eine andere Art, S. murdochi, vorstellen.]

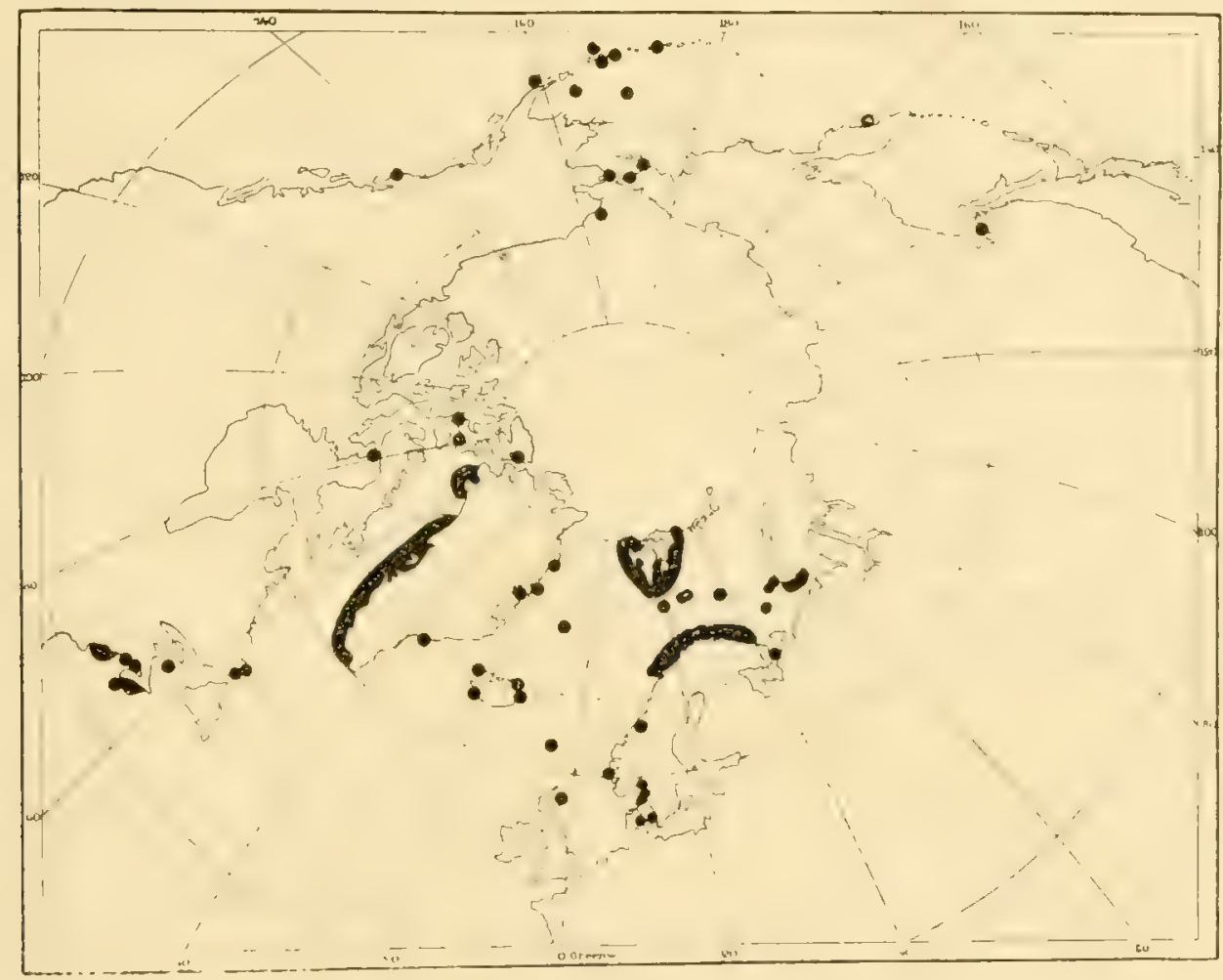

Fig. 1. Spirontocaris spinus.

Boreale Rogion:

Wostfin m a $\mathrm{xk}$ en und $\mathrm{L}$ of otengebiet: $S$. spinus lebt hier regelmâssig, wenigstens bis $67^{\circ}$ n. Br. (Lilljeborg 1851 und OHLin 1901: Tromsö; Sparre-Scineider 1884 und Aurivillius 1886: Kvaenangs. fjord; SARS 1886: Röst in den Lofoten; besonders NordgaARD 1905, DoNs 1915). Tielo der Fundorto liegen in den kalten, mehrero jedoch in den warmen Fjordon und an der offenen Küste. Wahrscheinlich ist dio Art nur in den boreoarktischen Gobieten von Norwegen häufig (nach DANIELssex [1861] ist sio sselten in den Lotfoten, etwas häufiger bei 'Tromsö, sehr häufig bei Vadsö)).

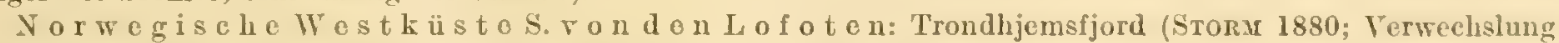
mit S. lilljeborgi wahrscheinlich ausgeschlossen). [Kristiansund und Bergen (DANIELsSEv 1859, 1861; iselten und nur in grosser Tiefe»); die Richtigkeit der Bestimmung kaum gesichert, obgleich $S$. lilljeborgi in derselben Abhandlung beschrieben wird.] Hardangerfjord (SARS 1872; wahrseheinlich richtigo Bestimmung, doch ron GRIEG [1914] nicht erwähut). - Die älteren Angaben (M. SArS) boziehen sich zweifellos grösstenteils auf $S$. lilljeborgi.

1 Bei den folgenden Arten zitiere ich in bezug auf die Verbreitung an den Küsten von Grönland nur dio Zusammenfassung von STEPHEXSEX (1913), sowie scine neueste, erst während der Drucklegung erschienene Arbeit (1916).

K. Sr. Pet. Akad. Hzmll. Banil 5t. N:o 7. 
S $\mathrm{k}$ a $\mathscr{\mathrm { C }} \mathrm{OT}$ a $\mathrm{k}:$ Aus älterer Zeit stammen ganz vereinzelto Funde an der sehwedischen Küsto: Väderöama,

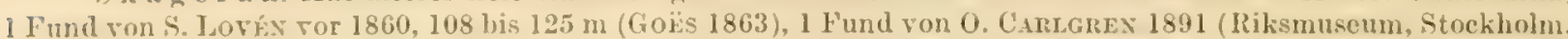
nach freundlicher Mittoilung von Dr. A. Molasner, der auch dio Richtigkeit der Bestimunng von Gö̈s hestätigt hat). Gullmarfjord und naho am Eingang, 1 Fund von Lovex vor 1860 (Gö̈s 1863), 1 Fund von WALM, August 1852, $45 \mathrm{~m}$ (LAGerberg 1908), I Fund von Aorivilus, Juli 1889 (APPELLÖF 1906, LAGErBerg 1908). In den Jetzten Jahren hat die schwedischo hydrographisch-biologischo hommission mehrero Funde gemacht: Gullmarfjord 1909, 2 Funde, 70-110 m (Bзӥrск 1911, 1913). Kosterrinne, 206, 175 his 216, $230 \mathrm{~m}$, Febr. 1911; ohno Tie. fenangale, Juli 1902. Offener Shagerak $58^{\circ} 5^{\prime}$ n. Br., $11^{\circ}{ }_{7}^{\prime}$ ö. Id., $132 \mathrm{~m}$, Febr. 1911; Skagen, $150 \mathrm{~m}$, 1905 (diese Fundo nach Mitteilung von Dr. A. MolaneEr). [Die von Tribou (1903) erwähnten Fiude dürften sich anf s.lilljeborgi bezichen.]

Ki at ziehen sich zwar auf S. lilljeborgi (HAXSEN 1908, STEPIENSEx 1910), da aber die beiden erwähnten Autoren den Fund hei Samsö unter S. spinus aufnehmen, kann die Richtigkeit der Bestimmung kaum angezweifelt werden.

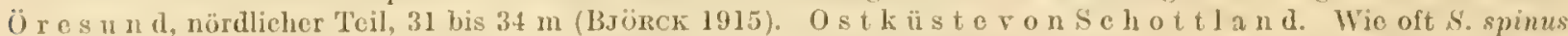
an den britischen Küsten beobachtet worden ist, kann nicht entsehieden werden; es ist, wie ApPELL̈̈ (1906) hersorheht, sehr wahrscheinlich, dass viele Angaben auf Vorwechslung anit S. lilljeborgi beruhen. Das der Originalboschreihung der Art zu Grunde liegende Exemplar ist jedoch, wio die Figur zoigt, eine vollständig typische spinus; es wurle mamong oysters on the Scottish coast gesammelt (SOWERBY 1806). Das ron LEACI (1815) abgebildete, an derselben Küste (Firth of Forth) gefundene Exemplar (Hipp. Soverbyi) seleint nach der Form des Rostrums uSw. ehenfalls zu S. spinus zu gehören, nähert sich aber in mehreren Hinsichten S. lilljeborgi; da es als simperfect» bezeichnet wird und die Figur also vielleicht teilweise nieht danach gezeichnet worden ist, kann die systematische Stellung nicht entschicden werden. BELL (1853) erwähnt zwoi in der Irischen See gefundene Exemplare; seine Figur stellt zwar eine typische S. spinus dar; dass sio nach einom irländischen Exemplar gezeichnet ist, wird jedoch nicht ausdrücklich gesagt und erscheint in der Tat sehr zweifelhaft, da ein Vorkommen in dieser Gegend änsserst ïberraschend wäro. HANSEN (1908) bezeichnet eine spätere Angabe (WALKER) über einen Fund in der Irischen Sēe als wahrscheinlich unrichtig; dasselbe gilt wohl von einer Angabe von KEMP 1910 (Testlich von Irland).

F i kïste von Island (HANSEN 1908).

Im $\mathrm{p}$ a $\mathrm{z}$ if is ch on G o biet ist diese Art nach SMrm (1880) und Lenz (1901) in noch wärmeren Go. genden beobachtet worden (Vaneouver Island, Queen Charlotte Islands). Die Bestimmung kann indessen nicht als sieher betrachtet werden, da in dieser Gegend naho verwandte, aber mehr südliche Arten existieren (S. lamelli. cornis, S. areuate, S. RATIBUN), die vielleicht in demselben Verhältnis zu S. spinus stehen wie S. lilljeborgi auf dor atlantischen Soite.

Bathymetrische Verbreitung. In arktischen Gebieten erstreckt sich die vertikale Verbreitung von etwa $7 \mathrm{~m}$ (HANsen 1887) oder weniger (STIMPsoN 1871: "low water mark") bis $400 \mathrm{~m}$ (nach unseren Beobachtungen). Oberhalb von etwa $25 \mathrm{~m}$ ist die Art iiberall nur ausnahmsweise erbeutet worden (HANsen, 1. c., SuITI 1879). Unterhalb von 150 und besonders von $200 \mathrm{~m}$ ist sie bisher nur ziemlich sporadisch gefunden worden; doch ist es kaum wahrscheinlich, dass der Eisfjord, wo sie bis 230 oder $250 \mathrm{~m}$ häufig ist, in dieser Beziehung eine Sonderstellung einnimmt.

In der borealen Region erstreckt sich die Verbreitung nach den vorliegenden Angaben von 40 bis $230 \mathrm{~m}$. Wahrscheinlich fehlt die Art ganz in seichterem Wasser und lebt meist erst in mehr als $50 \mathrm{~m}$ Tiefe. 


\section{Spirontocaris turgidil (KR.).}

syn. S. phippsi ( $\left.\mathrm{K} \mathrm{R}_{*}\right)$.

\section{Verbreitung im Eisfjord.}

Verzeichnis der Fundorte (s. Karte 1):

\begin{tabular}{|c|c|c|c|c|c|c|c|c|c|c|}
\hline $\begin{array}{l}\text { Nr. } \\
\text { der } \\
\text { Stat. }\end{array}$ & Ort unc & & Datur & & Tiefo & $\begin{array}{l}\text { Wasser- } \\
\text { temperatur }\end{array}$ & $\begin{array}{c}\text { Salz- } \\
\text { gehalt } \\
\% /{ }_{0}\end{array}$ & Bodenbeschaffenheit & Gerät & $\begin{array}{l}\text { Anzahl und } \\
\text { Grösse }\end{array}$ \\
\hline 5 & Safo Bay & - . & . . & .15 .7 & $2-8 m$ & {$\left[4-3,3\right.$ bis $\left.+4^{\circ}\right] \mathrm{H}$} & -- & $\begin{array}{l}\text { Stein und Kies mit La } \\
\text { minarien (und ein wenig } \\
\text { Schlamm). }\end{array}$ & Kl. Dredge & $\begin{array}{l}+\mathrm{Ex}, 16-22 \\
\mathrm{~mm}\end{array}$ \\
\hline 6 & $"$ & - . & . $\cdot$ & $\cdot \quad "$ & $40 \mathrm{~m}$ & - & - & Schlamm, etwas Stein & " & $\underset{\mathrm{mm}}{6 \mathrm{Ex}, 17-35}$ \\
\hline 8 & 3 & - & . . & . & $35 \mathrm{~m}$ & - & - & Fester Schlamm & $\Rightarrow$ & $\begin{array}{l}3 \mathrm{Ex}, 34-40 \\
\mathrm{~mm}(1 \text { q ovig. })\end{array}$ \\
\hline 9 & 3. & - . & . . & . ${ }^{\prime}$ & $5 \mathrm{~m}$ & $[+3,6$ bis $+4,4]]^{11}$ & - & Schlamm mit Steinen & " & $1 \mathrm{Ex}, 18 \mathrm{~mm}$ \\
\hline 22 & Ymor Bay. & . . & . . . & .20 .7 & $80-92 \mathrm{~m}$ & $\mid\left[0 \text { bis }-1^{\circ}\right]^{\mathrm{IV}} \mid$ & - & Loser Schlamm & $"$ & 1 Ex., $42 \mathrm{~mm}$ \\
\hline 23 & in $\quad$. & . & . . & . " & Etwa $100 \mathrm{~mm}$ & $\mid\left[0 \text { bis }-1^{\circ}\right]^{1 v} \mid$ & - & Foster Schlamm & $"$ & $\begin{array}{l}1 \text { Ex., } 46 \mathrm{~mm} \\
\text { († ovig) }\end{array}$ \\
\hline 25 & $"$ & - . & . . & . & $5-30 \mathrm{~m}$ & - & - & $\begin{array}{c}\text { Erst Kries mit Laminarien } \\
\text { dann loser Schlamm }\end{array}$ & " & $1 \mathrm{Ex} ., 20 \mathrm{~mm}$ \\
\hline 26 & " & . . & . . & . " & $78-50 \mathrm{~m}$ & $75 \mathrm{ml}:+1,7^{\circ}$ & - & Fester und zäher Schlamm & " & $\begin{array}{l}3 \mathrm{Ex}, 19,44, \\
51 \mathrm{~mm}(2 \text { f } \\
\text { ovig.) }\end{array}$ \\
\hline 27 & 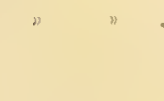 & . . & · $\cdot$ & & $30 \mathrm{~m}$ & - & 一 & $\begin{array}{l}\text { Kies und Stein mit Litho- } \\
\text { thamnion-Iŕsten und } \\
\text { Balants porcatus }\end{array}$ & 》 & $\begin{array}{l}16 \mathrm{Ex}, 19- \\
41 \mathrm{~mm}(5 \text { f } \\
\text { ovig. }\end{array}$ \\
\hline 92 & Nordarm & . & . & .19 .8 & $85-45 \mathrm{~m}$ & $42 \mathrm{~m}:+2,02^{\circ}$ & $\cdots$ & $\begin{array}{l}\text { Loser Schlamm mit Kies } \\
\text { und Sand. Am Ende der } \\
\text { Dredgung Steine und } \\
\text { Laminarien }\end{array}$ & Trawl & $\begin{array}{l}2 \mathrm{Ex} ., 22,37 \\
\mathrm{~mm}\end{array}$ \\
\hline 93 & Ekman Bay & $y$. & . & .20 .8 & $44-55 \mathrm{~m}$ & $+1,72^{\circ}$ & - & $\begin{array}{l}\text { Sehr zäher, stark roter } \\
\text { Sehlamm; etwas Stoin }\end{array}$ & " & $1 \mathrm{Ex}, 97 \mathrm{~mm}$ \\
\hline 114 & $"$ & - & . . & .22 .8 & $27-19 \mathrm{~m}$ & $19 \mathrm{~m}:-0,5^{\circ}$ & - & Zäher, roter Schlamm & Isil. Dredge & $\begin{array}{l}3 \mathrm{Ex}, 24-33 \\
\mathrm{~mm}\end{array}$ \\
\hline 117 & $\begin{array}{l}\text { Eingang in } \\
\text { son Bay }\end{array}$ & die & Dicl & .25 .8 & $29-27 \mathrm{~m}$ & {$\left[\right.$ otwa $\left.+2^{\circ}\right] \mathbf{x}$} & - & $\begin{array}{l}\text { Strauchförmiges Litho- } \\
\text { thamnion auf Schlamm- } \\
\text { boden }\end{array}$ & ” & $\begin{array}{l}3 \mathrm{Ex}, 26-29 \\
\mathrm{~mm}\end{array}$ \\
\hline 119 & $\begin{array}{l}\text { Eingang in } \\
\text { son Bay. }\end{array}$ & die & Dicl & 26.8 & $44-14 \mathrm{~m}$ & - & - & $\begin{array}{l}\text { Strauchförmiges Litho- } \\
\text { thamnion auf Schlamm- } \\
\text { boden }\end{array}$ & $\Rightarrow$ & $\begin{array}{l}4 \mathrm{Ex}, 18-33 \\
\mathrm{~mm}\end{array}$ \\
\hline 121 & $\begin{array}{l}\text { Eingang in } \\
\text { son Bay. }\end{array}$ & die & Dicl & k- 26.8 & $5 \mathrm{~m}$ & {$\left[+3,7^{\circ}\right] \mathrm{x}$} & - & $\begin{array}{l}\text { Schlamm mit Kies, Scha- } \\
\text { len und kleinen Steinen }\end{array}$ & " & $1 \mathrm{Ex}, 31 \mathrm{~mm}$ \\
\hline 76 & Billen Bay & - & . . & .13 .8 & $9-10 \mathrm{~m}$ & {$\left[\right.$ Etwa $\left.+5^{\circ}\right] \times$ xru } & - & $\begin{array}{l}\text { Kies, Stein (und Schalen) } \\
\text { mit Lithothamnion. (Ein } \\
\text { wenig Schlamm) }\end{array}$ & " & $1 \mathrm{Ex}, 20 \mathrm{~mm}$ \\
\hline 77 & $"$ & - & . & . $"$ & $9 \mathrm{~m}$ & {$\left[\text { Etwa }+5^{\circ}\right]^{\mathrm{xIIl}}$} & 一 & $\begin{array}{l}\text { Loser Schlamm mit Stein } \\
\text { Kies und Lithothamnion- } \\
\text { Bruchstiu:ken; oinzelne } \\
\text { Steine }\end{array}$ & $"$ & $1 \mathrm{Ex}, 24 \mathrm{~mm}$ \\
\hline 81 & $\begin{array}{l}\text { Eingang in } \\
\text { Bay . . }\end{array}$ & dio & Billc & .14 .8 & $26 \mathrm{~m}$ & {$\left[+1,82^{\circ}\right]^{\mathrm{x} v 1}$} & $\mid$ & $\begin{array}{l}\text { Strauchförmiges Litho- } \\
\text { thamnion; etwas Kies } \\
\text { und krustenförmiges Li. } \\
\text { thothamnion }\end{array}$ & : & 2 Ex., $26 \mathrm{~mm}$ \\
\hline 82 & Billen Bry & . & . & .15 .8 & $65 \mathrm{~m}$ & $-0,7^{\circ}$ & - & $\begin{array}{l}\text { Toils loser Schlamm, teils } \\
\text { fester Schlamm mit } \\
\text { Stoinon und Lies }\end{array}$ & " & $\begin{array}{l}+\mathrm{Ex} ., 23-42 \\
\mathrm{~mm}\end{array}$ \\
\hline
\end{tabular}




\begin{tabular}{|c|c|c|c|c|c|c|c|c|c|}
\hline $\begin{array}{l}\text { Nr. } \\
\text { der } \\
\text { Stat. }\end{array}$ & & rt und & Datum & Tiefo & $\begin{array}{l}\text { Wasser- } \\
\text { temperatur }\end{array}$ & $\begin{array}{l}\text { Salz: } \\
\text { gehalt } \\
\text { nou }\end{array}$ & Bodenbeschaffenheit & Gerät & $\begin{array}{l}\text { Anzahl und } \\
\text { Grösso }\end{array}$ \\
\hline 83 & Billen & Bay & $\ldots 16.8$ & $22 \mathrm{nn}$ & {$\left[\right.$ etwa $\left.+1,8^{\circ}\right]$ Xvin } & - & $\begin{array}{l}\text { Stark sandgemischter, } \\
\text { fester Schlamm mit } \\
\text { otwas Kies und Stein }\end{array}$ & Iil. Dredgo & $\begin{array}{l}3 \mathrm{Ex} ., 22-32 \\
\mathrm{~mm}\end{array}$ \\
\hline 85 & $\infty$ & 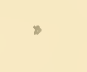 & $\cdots \cdot$ & $18-15 \mathrm{~m}$ & $\begin{aligned} & {[+3 \text { bis }} \\
+ & \left\{, 7^{\circ}\right] \times \text { VMI }\end{aligned}$ & - & $\begin{array}{l}\text { Stein und Kios mit Litho. } \\
\text { thamnion }\end{array}$ & 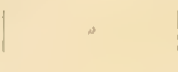 & I Ex., $27 \mathrm{~mm}$ \\
\hline S6 & , & s & $\ldots$, & $30 \mathrm{~m}$ & $+1,6$ & - & Kies und kleino Steino & " & $\begin{array}{l}8 \mathrm{Ex} ., 23-33 \\
\mathrm{~mm}\end{array}$ \\
\hline 101 & $\infty$ & 。 & $\cdots 14.8$ & $150-140 \mathrm{~m}$ & $140 \mathrm{~m}:-1,67$ & 34,43 & $\begin{array}{l}\text { Losor Schlamm mit Stei- } \\
\text { nen }\end{array}$ & 'Trawl & $1 \mathrm{Ex}, 32 \mathrm{~mm}$ \\
\hline 49 & Sassen & Bay, & Bank . 31.7 & $\begin{array}{l}24-19 \text { und } \\
19-28 \text { in }\end{array}$ & {$\left[+2\right.$ bis $\left.+3^{\circ}\right] x x$} & - & $\begin{array}{l}\text { Stein, Kies und Schulen } \\
\text { mit Lithothamnion }\end{array}$ & $\therefore$ & $\begin{array}{l}14 \mathrm{Ex}, 20- \\
35 \mathrm{~mm}(2 \text { ? } \\
\text { ovig. })\end{array}$ \\
\hline 44 & $\begin{array}{c}\text { Eingan } \\
\text { vent }\end{array}$ & $\begin{array}{l}n g \text { in } \\
\text { isuy }\end{array}$ & dio Ad- & $150-110 \mathrm{~m}$ & $128 \mathrm{~m}:+0,01^{\circ}$ & 34,54 & Loser Schlamm mit Kies & " & $1 \mathrm{Ex}, 32 \mathrm{~mm}$ \\
\hline 73 & Advont & t Bay & $\ldots 11.8$ & $35-30 \mathrm{~m}$ & $\begin{aligned} & {[+2 \text { bis }} \\
&\left.+2,7^{\circ}\right] \times x \times I\end{aligned}$ & - & $\begin{array}{l}\text { Balanus porcatus-Gemein- } \\
\text { schait. Kics und Stein. }\end{array}$ & líl. Dredge & $\begin{array}{l}33 \mathrm{Lx} ., 20- \\
40 \mathrm{~mm}(\mathrm{~T} \text { q } \\
\text { ovig.) }\end{array}$ \\
\hline 69 & Coles 1 & Bay . & $\ldots 8.8$ & $71 \mathrm{~m}$ & 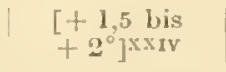 & - & $\begin{array}{l}\text { Kies, Stein und Schalen. } \\
\text { Etwas Lithothamnion }\end{array}$ & » & $\begin{array}{l}14 \mathrm{lix} ., 18- \\
41 \mathrm{~mm}\end{array}$ \\
\hline 126 & Fjordst & $\operatorname{tamm}$ &. .30 .8 & $47-31 \mathrm{~m}$ & {$\left[+2 \text { bis }+3^{\circ}\right]^{x \times v 1}$} & - & $\begin{array}{c}\text { Balanus porcatus-Gem. } \\
\text { Etwas Kios; Schlamm } \\
\text { inden Balanus-Kolonien }\end{array}$ & $"$ & $\begin{array}{l}11 \text { Ex., } 23- \\
34 \mathrm{~mm}(2) \text { 의 } \\
\text { ovig.) }\end{array}$ \\
\hline 130 & Green & Bay & $\cdots .30 .8$ & $40-45 \mathrm{~m}$ & - & - & Schlamm mit Algenresten & " & $\begin{array}{l}6 \text { Ex., } 37- \\
48 \mathrm{~mm}(2 \text { o } \\
\text { ovig.) }\end{array}$ \\
\hline 61 & , & " & $\cdots 4.8$ & $46-35 \mathrm{~m}$ & - & - & $\begin{array}{l}\text { Fies und Stein. Balanus } \\
\text { porcatus-Gemeinsch. }\end{array}$ & $\begin{array}{l}\text { (und Hum- } \\
\text { merkörbchen }\end{array}$ & $\begin{array}{l}9 \mathrm{Ex}, 32- \\
40 \mathrm{~mm}(3 \text { क } \\
\text { ovig.) }\end{array}$ \\
\hline 60 & " & , & $\cdots 3.8$ & $33 \mathrm{~m}$ & - & 一 & $\begin{array}{l}\text { Kies, Stoin und Schalen } \\
\text { mit Lithothamnion- } \\
\text { ISrusten; zahlreiche Ba. } \\
\text { lanus porcatus. Kolonion }\end{array}$ & | & $\begin{array}{l}35 \mathrm{Ex}_{2} 16- \\
40 \mathrm{~mm}(6 \text { \% } \\
\text { ovig.) }\end{array}$ \\
\hline
\end{tabular}
Längo).]

[Ausser in Eisfjord fanden wir S. turgida im Hornsund (Goẻs' Bay, 10.7, 27-40 m, 7 Ex., 22-40 mm

\author{
Frühore Fundo im Eis f jord:
}

Schwed. Exped. 1864: Safe Bay, 36 bis $54 \mathrm{~m}$, Schlamm; 1868: Advent Bay, 5,5 bis 9, 9 bis 18,36 bis $54 \mathrm{~m}$, Schlamm (Riksmuseum, Stockholm, nach A. MolaxDer). Norweg. Tordmeerexped. 1878: Ldveut Bay, $110 \mathrm{~m}$, 'Temp. + 0,7 , Schlamm (SArs 1886). Schwed. Exped. 1898: F'jordstamm vor Ki. BolleMan, 40 bis 50 in; $1899:$ Green 13ay, $110 \mathrm{~m}$; 1900: Coles Bay, $50 \mathrm{~m}$, Stein mit Schalen; 50 bis $100 \mathrm{~m}$, Stein (OnLiv 1901). IRuss. Exped. 1899: Green Bay, 98 bis $30 \mathrm{~m}$, 'Temp. $+3,0^{\circ}$, Grus und sandiger Schlamm (Brruld 1907).

Nach unseren Beobachtungen gehört S. turgida, wie S. spimus, zu den selteneren Nacruren der Eisfjords; bei der Anzahl von 31 Fundorten muss sie nichtsdestoweniger als eine allgemeine Art bezeichnet werden; insgesamt fanden wir $19+$ Exemplare.

An einigen Stationen wurde sie an festem Schlammboden erbeutet, meist jedoch an steinigem oder gemischtem Grund. Vor allem liebt sie die Balanus porcatus-Gemeinschaften und den Lithothamnion-Grund; oberhalb von $125 \mathrm{~m}$ fanden wir sie an fast allen (12) Stationen mit Balanus und strauchförmigem Lithothamnion.

Die vertikale Verbreit ung erstreckt sich im Eisfjord von $5 \mathrm{~m}$ bis 140 oder $150 \mathrm{~m}$. Ein genaues Studium der Dredgeergebnisse lehrt jedoch (s. die nachste- 
hende Ủbersicht), dass sie vorzugsweise den Tiefen zwischen etwa 20 und $75 \mathrm{~m}$ angehört. Oberhalb von $20 \mathrm{~m}$ wurde sie $6 \mathrm{mal}$ erbentet (dazu kommt möglicherweise St. 25, 5-30 m); da so zahlreiche Stellen in dieser Tiefe untersucht wurden, muss sie dort selten sein, obgleich nicht so selten wie die übrigen Spirontocaris-Arten. Dies geht auch aus der geringen Individuenzahl hervor; an 5 dieser Fundorte wurden nur vereinzelte Exemplare, am sechsten 4 Tiere erbeutet. In den Tiefen zwischen 20 und 70 oder $75 \mathrm{~m}$ scheint sie iiberall ungefähr gleich häufig zu sein; bei $71 \mathrm{~m}$ (St.69) fanden wir sie noch in verhältnismässig grosser Individuenzahl. Aus grösserer Tiefe stammen nur drei vereinzelte Exemplare (100, 150 bis 110 und 140 bis $150 \mathrm{~m}$ ); das Tier ist also hier fast seltener als im obersten Teil seines Verbreitungsbezirkes.

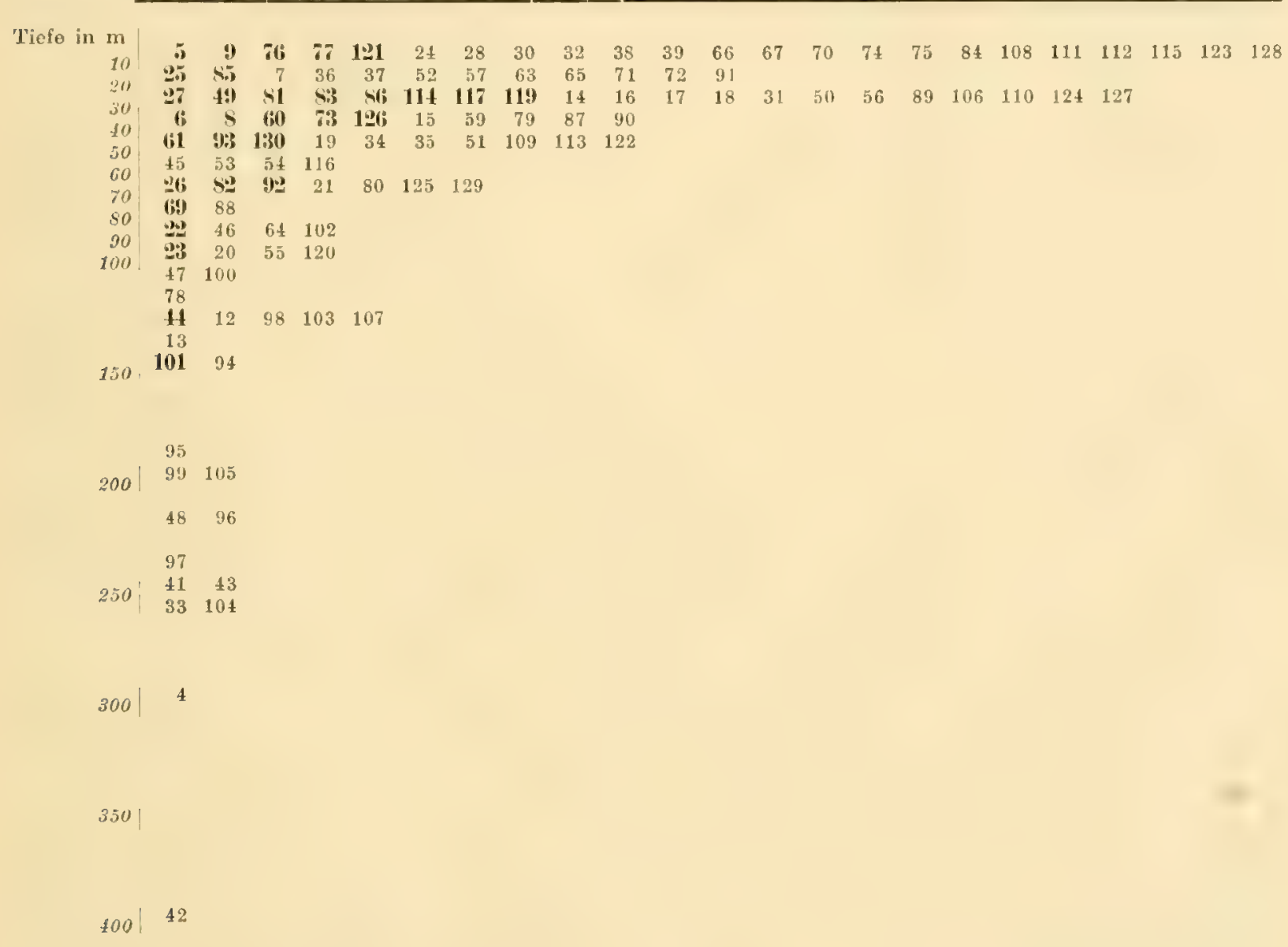

Die an den Fundstellen gemessenen Temperaturen schwanken zwischen etwa $+5^{\circ}$ und $-1,67^{\circ}$. Die Verteilung auf die zwischen diesen Extremen liegenden Temperaturen wird durch die nachstehende Übersicht veranschaulicht. Die Art ist demnach am häufigsten bei niedrig positiver Temperatur. Im wärmsten Wasser (mehr als $+3^{\circ}$ ) wurde sie 6 mal gefunden, mit einer Ausnahme (St. 5,4 Ex.) in je einem Exemplar; sie ist also hier zweifellos seltener als in etwas kälterem Wasser, obgleich der Unterschied nicht so stark in die Augen springt, wie bei z. B. S. polaris. In Wasser von negativer und sehr niedrig positiver (weniger als $+1,6^{\circ}$ ) 'Temperatur fanden wir sie noch 
seltener als im wärmsten Wasser (mehr als $+3^{\circ}: 18,7 \%$ der Stationen; $+1,0-+3^{\circ}$ : $28,9 \%$; weniger als $\left.+1,6^{\circ}: 13,8 \%\right)$.

\begin{tabular}{|c|c|c|c|}
\hline & 24 & 28 & 32 \\
\hline ()) & 85 & $1: 21$ & 7 \\
\hline 73 & (ry & 117 & $1 \geq 6$ \\
\hline (3) & 7:3 & 81 & LB \\
\hline 12 & 14 & 1fi & 17 \\
\hline 3 & 4 & 114 & 15 \\
\hline at & 55 & 88 & 100 \\
\hline
\end{tabular}

$\begin{array}{rr}38 & 39 \\ 50 & 52 \\ 4 & 18 \\ 47 & 13 \\ 40 & 95 \\ 105 & 120\end{array}$

\begin{tabular}{rr}
56 & 5 \\
19 & 30 \\
37 & \\
99 & 1 \\
34 & \\
\hline &
\end{tabular}

Aus diesen 'Tatsachen darf man jedoch nicht den Schluss zichen, dass S. turgida Temperaturen von +1 bis $+3^{\circ}$ bevorzugt. Was zunächst die Seltenheit im kältesten Wasser betrifft, muss schon die eigentümliche Lücke zwischen 0 (St. 44 : $+0,01^{\circ}$ ) und $+1,6^{\circ}$ (in diesem Falle die niedrigste Temperatur in der Reihe $+1-+1,9^{\circ}$ ) den Verdacht erwecken, dass sie durch andere Umstände bedingt ist. Die Tiefen- und Bodenverhältnisse dieser Stationen sind in der Tat derart, dass man ein häufigeres Auftreten von S. turgida nicht erwarten könnte; an den meisten bestand der Grund aus losem Schlamm und viele liegen dazu in Tiefen, wo die Art nicht oder nur selten lebt; die ibrigen Stationen zeigten festen Schlammboden, wo sie nicht häufig ist, keine einzige steinigen Grund. Die allgemeine Verbreitung zeigt iiberzeugend, dass sie unter den ausgesprochensten hocharktischen Bedingungen gut gedeiht.

Schwieriger ist es zu entscheiden, ob die Seltenheit in der obersten und zugleich wärmsten Zone des Fjords mit den Temperaturverhältnissen zusimmenhängt oder nicht. Aus hocharktischen Gegenden liegen mehrere Funde in ganz seichtem Wasser (s. besonders STEPIExisex 1912, 1913; im nördlichsten Teil von Nordostgrönland unter insgesamt 7 Fundorten 4 zwischen etwa 5 und $15 \mathrm{~m}$ ). Doch ist die Art auch an der Küste von Westgrönland mehrmals in ebenso seichtem Wasser gefunden worden (s. Stephensex 1913); ferner fehlt sie ja keineswegs in der obersten Zone des Eisfjords, ist nur seltener als in grösserer 'Tiefe. Es ist daher gar nicht ausgeschlossen, dass die Vertikalverbreitung iiberall dieselbe wie im Eisford ist. Man kamn aber die Vermutung wagen, dass die Art hei kälterem Oberflächenwasser häufiger bis nahe ans Lfer hinaufsteigt und folglich cine Sommertemperatur von weniger als $+3^{\circ}$ berorzugt.

Grösse, Fortpflanzung, Entwicklung. S. turgida ist der kleinste Decapod des Eisfjords. Die Weibchen tragen Eier schon bei einer Länge von 28 mm oder wenig mehr; das grösste von uns gefundene Exemplar (St. 130) misst 48 mm. Meist bleiben die Tiere etwas kleiner, und dies gilt nicht nur vom Eisfjord; fast alle früheren Autoren geben eine geringere Maximallänge an: OHLIN 1895: $45 \mathrm{~mm}, 1901$ : $41 \mathrm{~mm}$; Birula 1907: 47,5 mm; Stephensen (1912) fand die meisten Exemplare $45 \mathrm{~mm}$ lang; nach RATIBUN beträgt die approximative Länge $37,5 \mathrm{~mm}$; nur BIRULA erwähnt in seiner letzten Arbeit (1910) ein etwas grösseres, $52 \mathrm{~mm}$ langes Exemplar.

Eiertragende Weibchen finden sich unter den von St. 8, 23, 26, 27, 49, 73, 126, 130, 60 und 61 stammenden Exemplaren, also kaum an einem Drittel der Fuindstellen; eine nähere Betrachtung der Fundergebnisse zeigt, dass an den ibrigen Stationen entweder nur ganz kleine Lxemplare oder höchstens vereinzelte Weibchen von mehr als $30 \mathrm{~mm}$ Länge gefunden wurden. Die Eier befinden sich ausnahmslos in sehr frïhen Entrick- 
lungsstadien, sowohl bei den im Juli wie bei den am Ende des Sommers (bis 30. August) gefangenen Weibchen. Die hauptsächliche Entwicklung der Eier muss also im Herbst oder Winter erfolgen. Im nördlichen Norwegen scheinen die Eier erst im Herbst abgesetzt zu werden (s. Dons 1915).

\author{
Allgemeine Verbreitung. \\ (Fig. 2.) \\ śpitzbergongebiet:
}

Westspitzbergen: Hornsund, Belsund (OnLIN 1901, I3rula 1907, Doss 1915); Eisfjord (s. oben). [Forner Krörer 1842: "Spitzbergeny.] Nord westspitzbergen (HoEk 1882, SARS 1886, OHLIN 1901, Dons 1915). Ostspitzbergen: Storfjord und S. davon (Stebrixg 1900, Brrula 1907). Hopen Filand (Dows 1915).

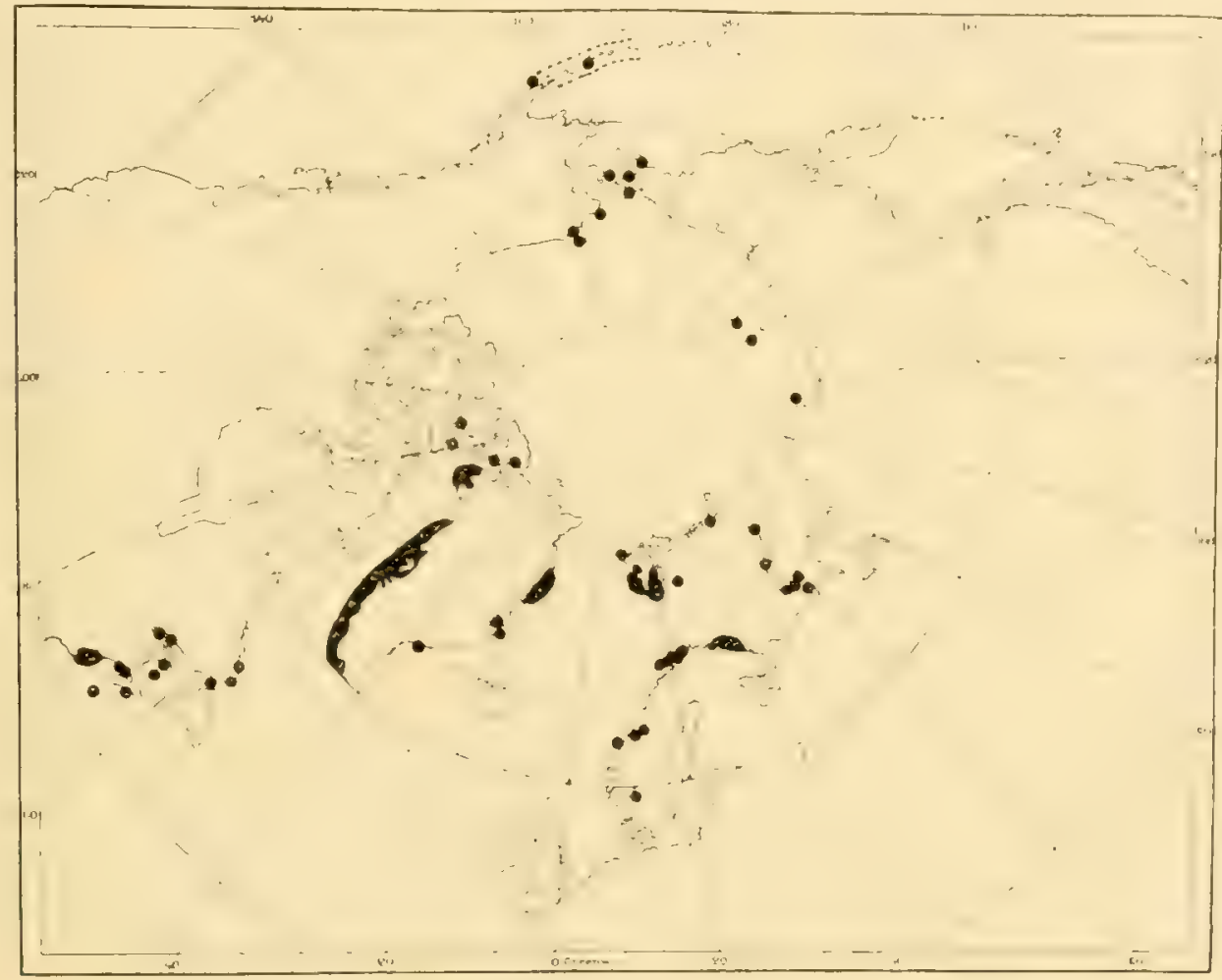

Fig. 2. Spirontocaris turgida.

Von Ostspitzbergen liegen also nur, sehr wenige Funde dieser Art ror. Dass DofLery sie nicht von ilort crwähnt, bedeutet jedoch nicht, dass sie von der Helgoland-Expedition nicht erbeutet wurde, sondern ist wahrscheinlich seiner eigentümlichen systematischen Auffassung zuzuschreiben; er betrachtet die beiden schon in frühen Stadien sehr scharf getrennten Arten S. turgida und S. spinus als identisch. Aber auch die schwedischo Expedition 1898 fand nach OrILIN (1901) S. turgida in Ostspitzbergen nicht. Daraus zieht BIrUhA (1907) den Schluss, dass diese Art ausser dem Storfjord, wo die Russischen Expeditionen sie auf vier Fundorten erbeutet haben, in Ostspitz. bergen gänzlich fehlt oder sehr spärlich vorkommt\%. Meiner Ansicht nach liegen keine Beobachtungen ror, die einen solchen Schluss wahrscheinlieh machen. S. turgida lebt (s. oben) vorwiegend oberhalb von 75 (nach HAxSFx nogar 50) $\mathrm{m}$, und die genannte schwedische Expedition untersuchte in Ostspitzbergen nur 7 Stationen in dieser 'liefe; 1 daron liegt im Storfjord, 2 nale der unteren Grenze $(60-70 \mathrm{~m})$. Wie wenig man bercehtigt ist, aus solchen negativen Ergebnissen allgemeine Schlüsse zu ziehen, geht schlagend aus dom Unstand herror, dass die er. wähnte Expedition in ganz Ostspitzbergen einschliesslich dem Storfjord S. spinus nur zweinal, s. gaimardii nur einmal fand; bide Arten sind dort allgemein. Da S. turgida in anderen hocharktischen Gegenden lhäufig gefunden worden ist und östlich von spitzbergen lebt, kann man nicht einsehen, wamm sie in Ostspitzbergen fehlen sollte. 


$$
\text { i) brige arktisehe und boreosthische liegion. }
$$

Sihirisches lismeer von den Nensihirischen Inseln his Ostaimyr (Bnela 1910) Jugor schar, harische Pforte.

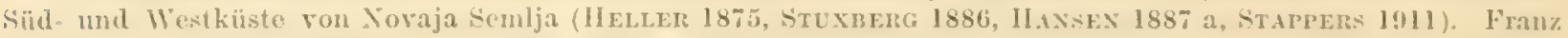
Josephs Land (Mires 1881, ScotT 1894). Weisses Heer (keine näheren Augahen, nielit auf der harte Fig. 2 he-

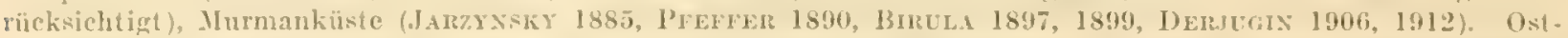

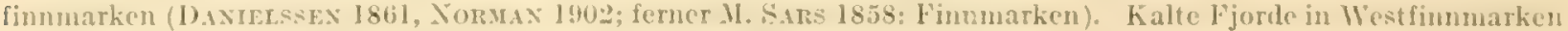
(s. unten). - Vorlost, siddost, West- und Fordwestgrönland (NTtruexsex 1913, 1916 und frühere Autoren).

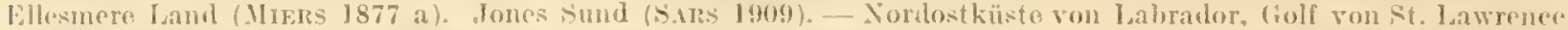

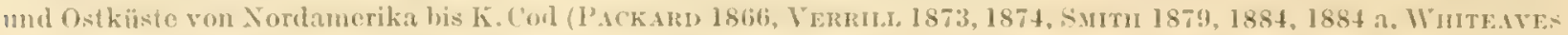

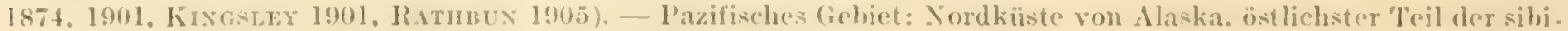
rischen Küste, Beringsstrasse, Beringsmeer, Aleuten (STIM

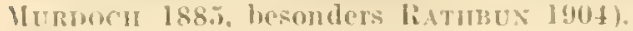

$$
\text { Boreale } \log \text { og ion: }
$$

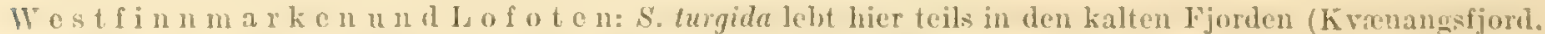

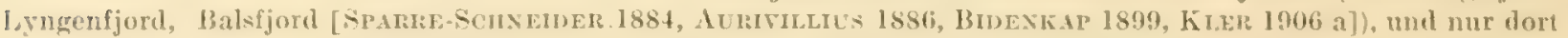
ist sie wohl allgemein, doch felit sio auch nicht in warmen (iebiet (siche besonders Doxs 1915).

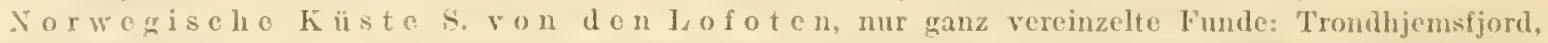
kparlich (STоRм 1878, 1880). „Wenigstens bis Kristiansund (KR̈̈rER 1842); Kistiansund, selten (DANIELSSEX

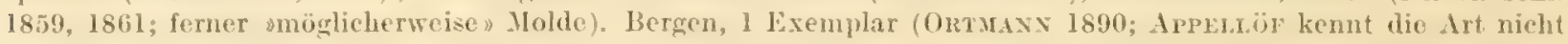
von dieser fiegend; da die Fundortsangahe vielleicht nicht sicher ist, habe ich diese Stelle nicht in der harte mit aufgenommen).

Sch wodischo S k agorak-K ï te: Koster, l Fxomplar von S. Lovex (ror 1860) gefunden (Gö̈s 1863). Doss (1915) vermutet mit Unrecht, dass diese Angabe auf cinem Misscerständnis beruht. In Reichsmuseum finden sich nach freundicher Mitteilung von Dr. A. MoLANDEn 3 Exemplare mit dor Aufschrift „Bohnslän n, wahrscheinlich ron Lovis gesammelt. BJörck (1911) verzeichnet drei Fundo dieser Art (Kostorrinne und offe. ner Skagerak), nach einer späteren Angabe (1913) können sio jedoch nicht als sicher angesehen werden.

Im pazifischen (robiot ist diese Art nach Surme (1880) weit südwärta gefunden worden (Qucen Charlotte Islands). Das einzige beobachtete Exemplar scheint jedoch eher zu S. ochotensis Braxis, zu gehören, welche allerdings sehr nahe verwandt mit $S$. turgida ist. Dofuery (1902) crwähnt $S$. turgida von Nord-Japan (Ost. kiiste von Jezso); auch dieso Ingabo dïrito bis auf woiteres als zwoifelhaft gelten.

Bathymetrische $V$ erbreit ung. Die vertikale Verbreitung erstreckt sich an den arktischen und boreoarktischen Küsten von ungefähr $5 \mathrm{~m}$ (s. besonders Stuxberg 1886, Hansen 1887, Stephensex 1912 und oben) bis höchstens $225 \mathrm{~m}$ (SMIтI 1879) (eine Angabe iiber einen Fund in $360 \mathrm{~m}$ Tiefe wird von Haxser als "certainly incorrect» bezeichnet). Wie HANSEN schon 1887 hervorhebt, ist die Art jedoch selten im unteren 'Teil dieses Verbreitungsbezirkes. Er setzt (auch 1908) die untere Grenze für das häufige Vorkommen bei 25 Faden $(45 \mathrm{~m})$; nach unseren Beobachtungen im Eisfjord kamn die Art, was wohl auch einige frühere Beobachtungen andenten (vgl. Sмiти 1879, Ohuin 1901), noch in einer Tiefe von etwa $75 \mathrm{~m}$ ziemlich häufig sein. Die Verbreitung in der Uferzone habe ich oben besprochen. Über die vertikale Verbreitung in der borealen Region ist wenig zu sagen (im 'Trondhjemsfjord nach STom auf Braunalgengrund), da das Tier dort nur ganz ausnahmsweise auftritt.

\section{Spirontocaris polaris (SAB.).}

syn. S. borealis (OWEX).

Dass Spirontocaris borealis (OWEN) nur das ältere Männchen von S. polaris (SAB.) darstellt, ist jetzt sicher erwiesen (s. z. B. APPELLÖF 1906), obgleich einzelne Autoren (DerJugra 1912) noch beide Species anführen. Auch an unserem reichlichen Material 
sind, wie die unten angeführten Notizen über die Form des Rostrums und der unteren Ecke des Thorakalschildes zeigen, alle Übergänge vorhanden zwischen einerseits den Weibchen und jüngeren Männchen, andererseits den älteren Männchen. Man sieht daraus, dass die Nännchen bis zu einer Länge von wenigstens 30, meist 35-42 mm das Aussehen der Weibchen behalten. Die Ersetzung des .Jugendkleides durch die boreatis-Tracht erfolgt meist bei einer Körperlänge von 35-45 mm; bei dieser Grösse findet man Exemplare, deren Rostralzähnchen und Frontolateraldorne nur wenig kleiner sind als beim Weibchen (St.98, 116), ferner solche, bei welchen sie mehr oder weniger klein oder rudimentär oder kaum mehr erkennbar sind. Es kommt jedoch nicht ganz selten vor, teils dass die Männchenmerkmale sehr früh auftreten (St. 27: 1 o $31 \mathrm{~mm}$ mit stark reduzierten Rostralzähnchen und Frontolateraldornen; St. 101: 1 typisches borealis-o $30 \mathrm{~mm}$ ), teils dass die Männchen ungewöhnlich lange Spuren der Jugendtracht behalten (St. 4: $65 \mathrm{~mm}$; St. 33: 55, $56 \mathrm{~mm}$; St. 98: $57 \mathrm{~mm}$; St. 48: 55-61 mm; St. 95: 58, 60, mm). Bei 42-45 $\mathrm{mm}$ haben die Männchen oft, bei $45-65 \mathrm{~mm}$ in der Regel, bei noch grösserer Länge ausnahmslos alle Weibchen-(Jungen-)Merkmale verloren.

\section{Verbreitung in Eisfjord.}

Verzeichnis der Fundorte (s. Karte 3):

\begin{tabular}{l|l|l|l|l|l|l|l}
\hline $\begin{array}{l}\text { Nr. } \\
\text { der } \\
\text { Stat. }\end{array}$ & Ort und Datum & Tiefo & $\begin{array}{c}\text { Wasser- } \\
\text { temperatur }\end{array}$ & $\begin{array}{c}\text { gehalt } \\
\%\end{array}$ & Bodenbeschaffenheit & Gerät \\
\hline
\end{tabular}

42 Srensksundstiefe $.257 \mid 406-395 \mathrm{~m} / 382 \mathrm{m:}+2,61^{\circ} \cdot 34,90$ Loser Schlamm

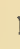

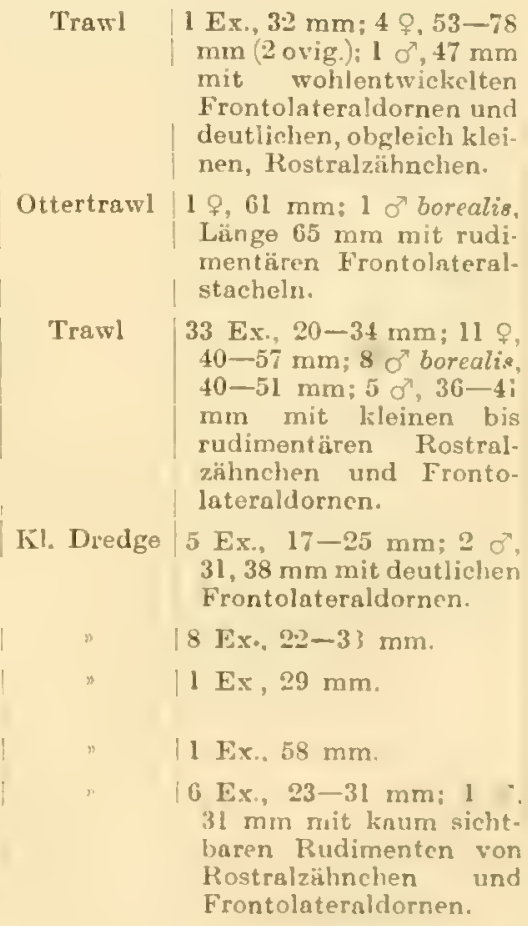

Trawl $1 \mathrm{Ex}, 32 \mathrm{~mm} ; 4$ ㅇ, $53-78$ mm (2 ovig.): $10,47 \mathrm{~mm}$ mit wohlentwickelten Frontolateraldornen und deutlichen, obgleich kloinen, Rostralzähnchen.

Ottertrawl | 1 \%, $61 \mathrm{~mm}: 1$ o borealis, Länge $65 \mathrm{~mm}$ mit rudimentären Frontolateral. stachelr.

Trawl 33 Ex., 20-34 mm; 11 ㅇ, $40-57 \mathrm{~mm} ; 8$ or borealis. $40-51 \mathrm{~mm} ; 5$ oे $36-4 i$ $\mathrm{mm}$ mit kleinen bis rudimentären Rostralzähnchen und Frontolateraldornen.

Kl. Dredge 5 Ex., 17-25 mm; $2 \sigma$ $31,38 \mathrm{~mm}$ mit deutlichen Frontolateraldornen.

8 Ex. 2:2-33 $\mathrm{mm}$.

$1 \mathrm{Ex}, 29 \mathrm{~mm}$.

1 Ex. $58 \mathrm{~mm}$

6 Ex. $23-31 \mathrm{~mm} ; 1$ $31 \mathrm{~mm}$ nuit knum sicht. baren Rudimenten von Rostralzühnchen und Frontolateraldornen. 


\begin{tabular}{|c|c|c|c|c|c|c|c|}
\hline $\begin{array}{l}\text { Nr. } \\
\text { der } \\
\text { Stat. }\end{array}$ & Ort und Datum & Tiefo & $\begin{array}{l}\text { Wasser- } \\
\text { temperatur }\end{array}$ & $\begin{array}{l}\text { Salz- } \\
\text { gehalt } \\
\% \% 0\end{array}$ & Bodenbeschaffenheit & Gerät & Anzahl und Grüsse \\
\hline
\end{tabular}

33 Fiordstnmm . . 23.7 263-256 m[+2bis $+2,0]$ ws - Loser Schlamm

41

21 Eingang in dio Tun- $71-65 \mathrm{~m} \quad-0,93^{\circ} \quad 34,29 \mid$ Loser Schlamm, stellen. dra Bay... 20.7,

94 Fjordstamm . . 21.8 $147-141 \mathrm{~m}, 140 \mathrm{~m}:-0,62^{\circ} \mid 34,49$ Loser Schlamm mit Stoinen

92

Nordarm -. . $19.5 \$ 5-45 \mathrm{~m} 12 \mathrm{~m}:+2.02^{\mathrm{c}} \quad$ - Loser Schlnmm mit IVies und Sand. Am Finde der Dredgung Steine und Laminarien

98 $27.8130-116 \mathrm{~m} 115 \mathrm{~m}:-0,82^{\circ} 34,40 \mid$ Loser Schlamm
99

102

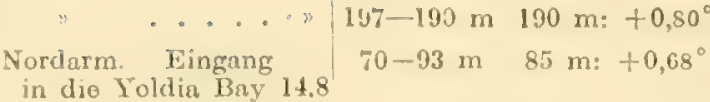
in die Ekman Bay 19.8

93 Ekman Bay . . . 20.8 Eingangindie Dickson Bay ... . 258 8
91 Nordarm. Eingung

116 | Nordarm. Vor dem
$11 \mathrm{~m} \quad\left[\right.$ otwa $\left.+3,7^{\circ}\right] \mathrm{vIIr}$

$$
44-55 \mathrm{~m} \mid+1,72^{\circ}
$$$$
57-60 \mathrm{~m}
$$$$
+1,2^{\circ}
$$

117

$$
\begin{aligned}
& \text { Eingang in die Dick. } \\
& \text { son Bay . . . } 25.8
\end{aligned}
$$

19 Eingang in dio Dickson Bay . . 26.8
34,72

34,25 Zäher und fester Schlamm mit vielen Steinen
'Trawl
4 Fx., 28-41 mm; 13 . $64-79 \mathrm{~mm}$ (4 ovif) ) 9 б Lorealis, $59-63 \mathrm{~mm} ; 2 \sigma^{\prime}$, $55,56 \mathrm{~mm}$ mit kaum sichtbnren Rudimenter von Rostralzähnchenund Frontolateraldornen.

9 ㅇ, $43-78 \mathrm{~mm}$ (4 ovig.) 3 of borealis, 60-64 mm. 2 Ex., 22, $30 \mathrm{~mm}$; \&, $47 \mathrm{~mm} ; 1 \mathrm{o}^{7}$ borcalis, 43 $\mathrm{mm}$.

4 Ex. 24-37 mm; 7 ; $47-57 \mathrm{~mm} ; 2$ on borealis, $54,59 \mathrm{~mm}$.

4 Ex., $32-42 \mathrm{~mm}$.

3 o, 24, 54, 62 (ovig.) $\mathrm{mm}$; $10,40 \mathrm{~mm}$ mit deutlicher Bezahnung des Rostrums und grossen Frontolateraldomen: $10^{7}$, $57 \mathrm{~mm}$ mit schwsch angedeuteten Rostralziihnchen und Fronto. lateraldornen fast ebenso kräftig wio boim ?.

1 Ex., (ovig.) $67 \mathrm{~mm}$.

3 ค, 40-44 $\mathrm{mm} ; 1 \mathrm{o}^{7} \mathrm{bo}$. realis, $42 \mathrm{~mm}, 1$ ơ $35 \mathrm{~mm}$, mit undeutlichen Rostra]. zühnchen und rudimentïren Frontolateral. dornen.

KI. Dredge 1 Ex., $25 \mathrm{~mm}$.

Loser Sehlamm mit Kies und Sand; oinige Steine mit Lithothamnion

Sehr zäher, starls roter Schlamm. Etwas Stein. Kies und Stein

Trawl

2 ㅇ, 38, $55 \mathrm{~mm}$

Iil. Dredgo

1 Ex., $24 \mathrm{~mm}$ 14 우 $39-$ $54 \mathrm{~mm}$ ( 3 ovig.); $3 \sigma^{7}$ bo. realis, $44-52 \mathrm{~mm} ; 40$, $39-45 \mathrm{~mm}$ mit wohl. entwickelten Rustralzïhnchen (kaum kleiner als beim f) und kleinen aber deutlichen Fronto. lateraldornen.

2 Ex., 30, $38 \mathrm{~mm}$.

Strauchförmiges Lithothamnion nuf Schlammboden

Strauchfürmiges Lithothamnion auf Schlammhoden

9 Ex. 30-36 $\mathrm{mm}: 11$ ㅇ․ $40-55 \mathrm{~mm}$ : 12 o borealis, $42-49 \mathrm{~mm}: 1 \sigma^{7} 41 \mathrm{~mm}$ olne Frontolateraldornen aber mit schwachen Spurenvon Rostralzühnchen

1 Ex., $32 \mathrm{~mm}$. 


\begin{tabular}{|c|c|c|c|c|c|c|c|}
\hline $\begin{array}{l}\text { Nr. } \\
\text { der } \\
\text { Stat. }\end{array}$ & Ort und Datum & Tiefe & $\begin{array}{l}\text { Wasser. } \\
\text { temperatur }\end{array}$ & $\begin{array}{c}\left.\mathrm{Sa}_{\mathrm{a}}\right]_{\mathrm{z}} \\
\text { gehalt } \\
{ }_{0}\end{array}$ & Bodenhescladfenheit & Gerät & Anzahl und Grösso \\
\hline
\end{tabular}

79 Billen Bay -.. 13.8 $32-40 \mathrm{~m} \mid[+1,5$ bis +2$] \times \mathrm{v} \mid-$ Grosse Steine mit strauch $\mid$

80 Eingang in dio Billen 14.

81 Eingang in dio Billen

Bay..... 14

82 b Billen Bay ... 15.8

$69-64 \mathrm{~m} \quad 69 \mathrm{m:}+1,5^{\circ}$

$26 \mathrm{~m} \quad+1, \mathrm{~s} 2^{\circ} \mathrm{xvI}$

6วั $\mathrm{n}$

$-0,7^{\circ}$

83 $\left[\text { etwa }+1,8^{\circ}\right]^{\mathrm{x} y I I}$
Loser Schlanm (nit ein wenig Sand und Kies)

33,77 Strauchförmiges Litho. thamnion; etwas Kíos

Fester Schlamm mit Steinen und Kies

Sandgemischter, fester Schlamm mit Kies und Steinen
86

101

43 Sassen Bay, Bank vor dem Eingang in die Gips Bay . . . 31.7

51

Tempel Bay . . 30.7 $45-43 \mathrm{~m}$

48

Ostarm .... 31.7 $199-226 \mathrm{~m}$

\section{$+2,5^{\circ}$} $210 \mathrm{m:}+1,27^{\circ} \mid 34,72$ Loser Schlamm
Kies und kleine Steine Loser Schlamm mit Steinen

Stein, Kies und Schalen mit Lithothamnion
Trawl

$$
1 \text { \%, } 48 \mathrm{~mm} ; 2 \text { o borealis. }
$$
$30,67 \mathrm{~mm}$

27 Ex. 21-39 $\mathrm{mm} ; 7$ 웅 $42-53 \mathrm{~mm}$ (2 ovig.); $30^{7}$ borealis, 42- $45 \mathrm{~mm} ; 10$. $39 \mathrm{~mm}$ mit deutlichen Rostralzähnchen - und kleinen, aber deutlichen Frontolateraldornen.

\section{1 ?, $48 \mathrm{~mm}$.}

Trawl 6 ㅇ, $38-73 \mathrm{~mm}$ (2 ovig.); $3 \sigma^{4}, 55-61 \mathrm{~mm}$ mit kleinen aber deutlichen bzw. äusserst kleinen IRostralzähnchen und kleinen Frontolnteruldornen.

1 ơ borealis, $62 \mathrm{~mm}$.

3 Ex., 33-37 mm; 1 ㅇ․ $45 \mathrm{~mm}$.

Kl. Dredge $19 \mathrm{Ex}, 26-37 \mathrm{~mm} ; 7$ 으, $10-49 \mathrm{~mm}$ (2 ovig.): 2 or borealis, $41,43 \mathrm{~mm}$.

Trawl $20 \mathrm{Ex}, 23-12 \mathrm{~mm} ; 14$. 42-72 mm (2 ovig.); 10 , $4: 2 \mathrm{~mm}$ mit grossen Rostralzïhnchen und Frontolateraldornen; $12 \sigma^{7}$ borealis, $54-73 \mathrm{~mm} ; 3 \sigma^{\circ}, 52$, $58,60 \mathrm{~mm}$ mit lilcinen. aber deutlichen Rostral. zähnchen und Frontolaternldornen.
69

Coles 13ay . . . $8.8 \quad 71 \mathrm{~m}$

96

126

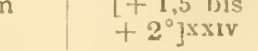

Fjordstamm . . 2.2.8 230 - otwa $208 \mathrm{m:}+2,56^{\circ}$

$200 \mathrm{~m}$

.. $30.8+7-31 \mathrm{~m}\left[+2 \text { bis }+3^{\circ}\right]^{\mathrm{x} \times \mathrm{I}}$

- Kies, Stein und Schalen.|Ki. Dredge Etwas Lithothamnion

34,76 Schlamm mit Stein und Trawl $12 \mathrm{Ex}, 2.2-42 \mathrm{~mm}$. Kies

Balanusporcatus-Gemoin.: Kl. Dredge 31 F.x. 24-40 mm. schaft 


\begin{tabular}{|c|c|c|c|c|c|c|c|c|}
\hline $\begin{array}{l}\text { Nr. } \\
\text { der } \\
\text { Stat. }\end{array}$ & Ort und & Datum & Tiefo & $\begin{array}{l}\text { Wasser- } \\
\text { tomperatur }\end{array}$ & $\mid \begin{array}{c}\text { Salz. } \\
\text { gehalt } \\
\% \text { \% }\end{array}$ & Bodenbeschaffenheit & Gerät & Anzahl und Grösse \\
\hline 129 & Fjordstamm & $\cdots 30.8$ & $65 \mathrm{~m}$ & - & -. & $\begin{array}{l}\text { Sandgemischter Schlamm } \\
\text { mit Kies und Algen. } \\
\text { resten }\end{array}$ & Iś. Dredge & 4 Ex., $31-36 \mathrm{~mm}$ \\
\hline 130 & Green Bay & . 30.8 & $40-45 \mathrm{~m}$ & - & - & Schlamm mit Algenresten & & $\begin{array}{l}12 \text { Ex., } 25-40 \mathrm{~mm}: 7 \\
42-60 \mathrm{~mm}(1 \text { ovig }) ; 2 \\
\text { borealis. } 43,49 \mathrm{~mm} .\end{array}$ \\
\hline 59 & , & . 3.8 & Etwa $40 \mathrm{~m}$ & - & - & $\begin{array}{l}\text { Sehr loser Schlamm mit } \\
\text { 'T'eilen von Landpflan- } \\
\text { zen. }\end{array}$ & , & | $3 \mathrm{Ex} ., 33-39 \mathrm{~mm}$ \\
\hline 61 & $\Rightarrow$ & $\cdots 48$ & $46-35 \mathrm{~m}$ & - & - & $\begin{array}{l}\text { Ties und Stoin. Balanus } \\
\text { porcalus-Gemeinsch. }\end{array}$ & $\nu$ & 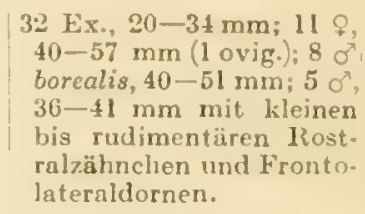 \\
\hline
\end{tabular}

[Ansser im Eisford fanden wir S. polaris im Ilornsund (Goes' Bay, 10.7, 10 bis 35 m, Schlamm und Kies, 4 kleine Ex.]

Iv $r$ ï hero Fu $u$ de im $E$ is $f$ jord:

Schwed. Exped. 1858-1873: Safe Bay, 36 bis $54 \mathrm{~m}$ und ohmo T'lefenangahe, Sehlamm; Skans Bay (Billen Bay), $27 \mathrm{~m}$, Schlamm; Advent Bay, 5,5 bis 9, 9 bis $18 \mathrm{~m}$, Schlamm; (treen 13ay, 9 bis $97 \mathrm{~m}, 36 \mathrm{~m}$, Schlamm; Lis. fjord ohne Lokalangabe, 45 bis $90 \mathrm{~m}$, Schlamm (Riksmuscum, Stockholm, nach $\Lambda$. MoLAxDER).

Norweg. Nordmeer-Exped. 1878: Ldvent Bay, $110 \mathrm{~m},+0,7^{\circ}$, Sehlamm (SARs 1886). Schwed. Lxped. 1890: Green 13ay, 35 bis $42 \mathrm{~m}$ (KLINCKowströs 1892). Helgoland-Exped. 1898: Fjordstamm, 210 bis $240 \mathrm{~m}$, Schlamm mit wenigen Steinen; Olga-Exped. 1898: Fjordstamm, vfast an derselben stelles (wie oben) und 145 bis $180 \mathrm{~m}$ (DofLeIN 1900; Angaben viclfach widersprecheud; nach der Stationskarte und der Kartenskizze S. 35s zu urteilen liegt der Fundort vam Eingang des Eisfjords》 vor der Coles 13 ay, also mindestens $20 \mathrm{~km}$ vom Iingang). Schwed. Exped. 1898: Srensksundstiefe, $400 \mathrm{~m}$; Fjordstamm, 40 bis $50 \mathrm{~m}$; Nordarm, $36 \mathrm{~m}$, Lithothamnion; 1900: Colos Bay, $100 \mathrm{~m}$, Stein; 50 bis $100 \mathrm{~m}$. Stein; vor dem Eingang in die Coles Bay, 150 bis $200 \mathrm{~m}$; Green Bay, 10 bis $80 \mathrm{~m}$, Stein (OmLin 1901). Russ. Exped. 1900: Fjordstamm, $205 \mathrm{~m},-0,8^{\circ} ; 243 \mathrm{~m},-0,8^{\circ}$; Billen Bay, 142 bis $133 \mathrm{~m},-1,9^{\circ}$, Schlamm mit Steinen (Birula 1907). Belgica-Exped. 1905: Green Bay (Grieg 1909 [S. pu. siola; s. unten S. 28]).

Wir fanden $S$. polaris an nicht weniger als 43 Stellen; also an einem Drittel der untersuchten Stationen. Ein noch schlagenderes Bild ron der grossen Hägfigkeit dieser Art im Eisford erhält man, wenn man die Anzahl der Fundorte mit der Anzahl der in den von $S$. polaris bewolmten Tiefen (mehr als $10 \mathrm{~m}$ ) gelegenen Stationen vergleicht; der Prozentsatz der Fundorte beträgt dann etwa 45. Zusammen wurden 563 Exemplare gesammelt.

Wie die übrigen Arten der Gattung liebt anch S. polaris mehr oder weniger harten B o d e $n$. Im Eisfjord findet man sie besonders an mit Stein oder Kies gemischtem Schlammgrund; reiner Steimboden ist ja selten in den von dieser Art bewohnten Tiefen, und es ist ohnehin nicht unmöglich, dass sie den gemischten Grund bevorzugt. Sehr häufig und oft in grosser Individuenzahl fanden wir die Art unter Lithothamnion (7 Stationen von 9) und Balanus-porcatus-Kolonien (5 Stationen von 7). S. polaris lebt jedoch auch auf losem Schlammboden, besonders in grösserer Tiefe, wo dieser uberwiegt, und ist jedenfalls häufiger auf solchem Grund als S. gaimardii und die meisten ibrigen Spirontocaris-Arten.

Die vertikalo Verbreitung (s. die nachstehende Ubersicht) umfasst fast alle im Eisfjord vorkommenden Tiefen. In der obersten Uferzone (bis etwa $10 \mathrm{~m}$ ) fehlt $S$. polaris jedoch nach unseren Beobachtungen vollkommen, und zwischen 10 und 
$20 \mathrm{~m}$ tritt sie nur ganz sporadisch auf; wir fanden sie dort nur an einer Station (oder viclleicht zwei; St. 25, 5-30 m), und nur in einem vereinzelten, jugendlichen Exemplar. Schon bei etwa 20 oder wenigstens $25 \mathrm{~m}$ ist sie häufig und von dort an bleibt sie allgemein bis in die grösste Tiefe des Fjords. Ob sie iiber diese ganze Zone gleichmässig verbreitet ist oder in gewissen Tiefen überwiegt, lässt sich nach unseren Beobachtungen nicht sagen; jedenfalls ist sie wohl nicht seltener unterhalb von $200 \mathrm{~m}$ (7 von 9 Stationen) als weiter oben.

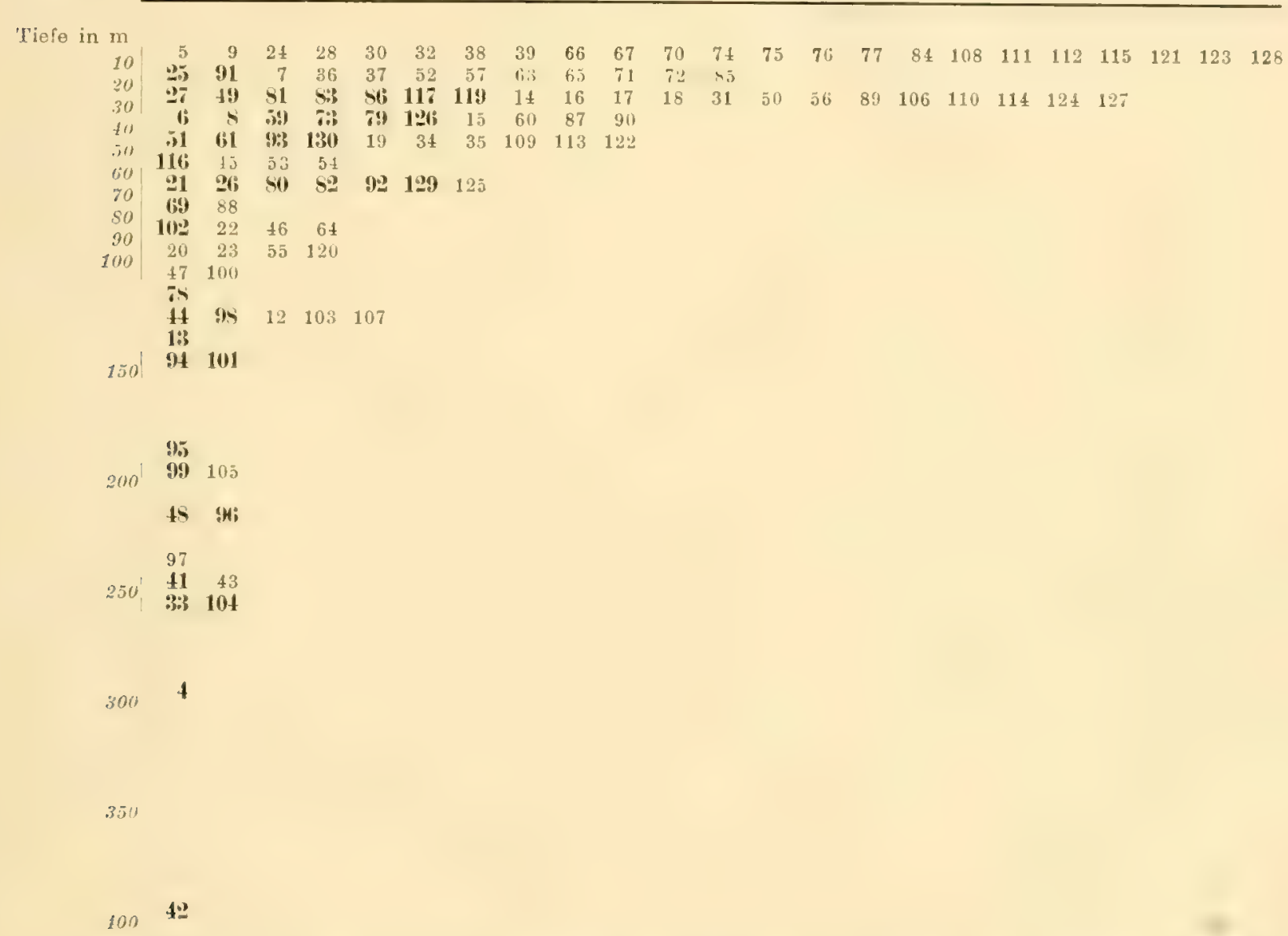

Zwischen der Tiefe des Aufenthaltsorts und der Körpergrösse besteht ein deutlicher Zusammenhang; die Grösse wächst mit zunehmender Tiefe. An den 28 in geringerer Tiefe als $100 \mathrm{~m}$ gelegenen Stationen wurden zusammen 359 Exemplare gefangen; ein einziges davon (St. 130 ) ist $60 \mathrm{~mm}$ lang, 4 messen $56-58 \mathrm{~mm}$, die uibrigen höchstens $55 \mathrm{~mm}$. Die 12 hier gefundenen eiertragenden Weibchen haben eine Länge von 43 bis $60 \mathrm{~mm}$ (8 davon $43-16 \mathrm{~mm}$ ).

9 Stationen liegen in Tiefen zwischen nahezu 200 (188-181) und $400 \mathrm{~m}$. An einer derselben (St. 96) wurden nur jugendliche Exemplare erbeutet. An den iibrigen fingen wir zusammen 109 Exemplare; die Maximalgrösse beträgt an den verschiedenen Stationen $62,65,67,72,73,78,78,79 \mathrm{~mm}$. Die Exemplare von 62 und $65 \mathrm{~mm}$ Länge (St. 104, 4) sind ja nicht viel grösser als in seichtem Wasser, es handelt sich aber um Männchen; an St. 48 fanden wir nur ein einziges Exemplar (von $67 \mathrm{~mm}$ Länge); an allen Stationen, 
ron welehen wir mehrere Exemplare besitzen, beträgt die Maximallänge wenigstens 72 $\mathrm{mm}$. Die eiertragenden Weibehen sind 15 an der Kahl und haben eine Länge von 62 bis $78 \mathrm{~mm}$.

An den 6 Stationen in mittleren 'Tiefen $(110-150 \mathrm{~m})$ fanden wir 83 Exemplare. [n ỉnrer Grösse halten sie die Mitte zwischen den oben besprochenen Extremen oder sie stimmen mit den grössten in seichterem Wasser gefundenen Exemplaren ïberein. Die Naximallänge beträgt 32 (ein cinziges Exemplar), 45, 57, 57, 62, 67 mm. Nur ein eiertragendes Weibchen wurde hier gefangen (St. 98); es hatte eine Länge von $62 \mathrm{~mm}$.

Aus den angefuhrten Messungen geht liolgendes hervor. Erstens, dass in Tiefen von weniger als $100 \mathrm{~m}$ und sogar noch bis $150 \mathrm{~m}$ auffallend oft nur kleine, nicht geschlechtsreife (oder sterile) Exemplare gefangen wurden. Z $Z$ e it ens, dass oberhalb von $100 \mathrm{~m}$ die Körperlänge auch bei geschlechtlich tätigen Individuen nie mehr als $60 \mathrm{~mm}$, meist etwas weniger beträgt. D rittens, dass an den meisten Stationen der grössten Tiefen (mehr als $150 \mathrm{~m}$ ) eiertragende Weibchen gefunden wurden. $V$ i ertens, dass die Körperlänge in diesen Tiefen durchschnittlich viel grösser ist; die Maximalgrösse schwankt meist zwischen 67 und $79 \mathrm{~mm}$, und an jeder Station wurden in der Regel mehrere bis viele Exemplare von mehr als $60 \mathrm{~mm}$ angetroffen. F ii $n \mathrm{ft}$ e $n \mathrm{~s}$, dass in seichtem Wasser die Weibchen, wenigstens sehr oft, schon bei einer Länge ron 43 bis $45 \mathrm{~mm}$ Eier legen.

Die letzte Tatsache wirde ein bedeutend erhöhtes Interesse erlangen, wemn sie durch die weitere Schlussfolgerung vervollständigt werden könnte, dass die weibliche Geschlechtstätigkeit in grösserer Tiefe später eintritt. Auf den ersten Blick scheinen unsere Funde auch den Beweis dafür zu bringen; die eiertragenden Weibchen der tieferen Stationen (unterhalb von 180 und sogar von $100 \mathrm{~m}$ ) sind ausnahmslos grösser als $60 \mathrm{~mm}$, und die kleineren lixemplare sind durchwegs steril. Doch ist hierbei grosse Vorsicht geboten, besonders weil wir in der ljordtiefe iberhaupt nicht viele kleinere Weibehen fingen; ferner sind ja auch in seichterem Wasser keineswegs alle oder gar die meisten 34-60 mm langen Weibchen eiertragend. Die Sammlungen der Kolthoff-Expedition 1900, die ich zum Vergleich untersucht habe, zeigen in den meisten Fällen dasselbe Verhalten, aber beweisen aus demselben Grund nichts Sicheres, und in einer aus $300 \mathrm{~m}$ Tiefe (Nordostgrönland) stammenden Probe finde ich zwei eiertragende Weibchen von nur 52 und $55 \mathrm{~mm}$ Länge. Soviel dürfte jedoch äusserst wahrscheinlich sein, dass die weibliche Geschlechtstätigkeit in seichtem Wasser of $t$ bei einer Länge von nur $43-45 \mathrm{~mm}$, in tiefem Wasser $m$ e is t erst bei einer Länge von mehr als 50, in der Regel vielleicht mehr als $60 \mathrm{~mm}$ eintritt.

Die Trage nach der Ursache dafür, dass die Körpergrösse mit der Tiefe zunimmt, ist ziemlich verwickelt. Nach den Verhältnissen in andern Gegenden wiirde man voraussetzen, dass die Grösse durch die ungiustige Einwirkung von warmem Wasser herabgesetzt werde. Dass die Grösse durch diesen Faktor beeinflusst werden kann, geht daraus hervor, dass die borealen Individuen, welche stets in beträchtlicher Tiefe leben, nie eine bedeutendere Grösse erreichen. ${ }^{1}$ Aber auch die kleinere Differenz zwischen Wasser

Iestimmte Angaben über die Durchsclnits. und Maximalgrüsse in der borealen liegion weruen von keinem Autor mitgoteilt; Appeüir (1906, S. 121) sagt nur: win den arktischen Gegenden erreicht die Art eine bedeutendero 
von negativer und solchem von niedrig positiver (bis etwa $+3^{\circ}$ ) 'l'emperatur wirkt auf die Grösse ein. An den grönländischen Küsten ist nach HANSeN (1908) die Art, wie im Eisfjord, grösser in tiefem als in seichtem Wasser, bei Ostgrönland sind aber die Seichtwassertiere »on the whole larger» als bei Westgrönland. Die Richtigkeit dieser Behauptung wird leider durch keine Angaben von Massen gestuitzt, ich habe mich aber durch Untersuchung des von der Kolthoff-Expedition 1900 in Nordöstgrönland eingesammelten Materials von ihrer Richtigkeit uberzeugt. Von St. 17 (s. OHLIN 1901), 12 bis $35 \mathrm{~m}$ Tiefe, finden sich zwei Exemplare von 67 und $68 \mathrm{~mm}$ Länge, und an St. 22, 12 bis $18 \mathrm{~m}$ Tiefe, wurde ein einziges, $74 \mathrm{~mm}$ langes Individuum erbentet. Auch im sibirischen Eismeer hat BirulA (1910) verhältnismässig grosse Exemplare (67 und $70 \mathrm{~mm}$ Länge) in seichtem Wasser gefangen.

Im Eisfjord kann man jedoch unmöglich irgend eine Beziehung zwischen der Grösse und den Temperaturverhältnissen konstatieren. Von den 8 in mehr als $180 \mathrm{~m}$ Tiefo gelegenen Stationen, an denen grosse Exemplare gefunden wurden, zeigten 4 - und dort wurden die grössten Tiere erbeutet - eine Temperatur von etwa $+2,5^{\circ}$, an den übrigen war die Temperatur $+1,62^{\circ},+1,27^{\circ},+0,80^{\circ},-0,11^{\circ}$; an den 28 Stationen oberhalb yon $100 \mathrm{~m}$ war sie nicht höher, meist niedriger (an 4 der Stationen, an denen wir eiertragende Weibchen fanden, schwankte sie zwischen $+1,2$ und +2 bis $\left.3^{\circ}\right)$. Die Berücksichtigung des allgemeinen hydrographischen Zustands gibt dasselbe Resultat: in der Tiefe des Fjordstamms und der Hauptarme iiberall atlantisches, mehr oder weniger warmes Wasser $\left(+1,7\right.$ bis $\left.+2,7^{\circ}\right)$, darüber eine kältere intermediäre Schicht (s. Sv. Hydrogr. Komiss. 1910 und meine Echinodermenarbeit). Auch im Winter kann das Tiefenwasser nicht kälter als die ganze Schicht 20-100 m sein. Vorläufig erscheint mir daher die Annahme unabweisbar, dass die Körpergrösse durch versehiedene Faktoren beeinflusst wird. Unter giunstigen Bedingungen erreichen die Tiere eine bedeutendere Grösse, und günstig wirken in dieser Beziehung teils (Eisfjord und wohl alle arktischen, auch hocharktischen Gegenden) grosse Tiefe des Wassers kombiniert mit nicht zu hoher (höchstens $+3^{\circ}$ ) Temperatur, teils (hocharktische Gegenden) sehr niedrige (negative) und konstante Temperaturen. W i e die Tiefenverhältnisse auf diese Weise die Grösse beeinflussen, lässt sich natürlich nicht sagen; wenn man von einer "giinstigen" Einwirkung spricht, darf man auch nicht vergessen, dass diese Worte nur eine Umschreibung für unbekannte Vorgänge sind; es ist z. B. denkbar, dass in geringer Tiefe die Geschlechtsreife friher eintritt - dies scheint ja wirklich der Fall zu sein - und dass die Tiere daher stets kleiner bleiben.

Mit dem ganzen Körper nehmen auch die Eier an Grösse zu; der grösste Durchmesser ( 2,7 $\mathrm{mm}$, bei den kleinen Tieren der oberen Wassersehichten 1,5 $\mathrm{mm}$ bis höchstens $2 \mathrm{~mm}$.

Andere Unterschiede habe ich nicht auffinden können. Die Bedornung des Rostrums, deren Ausbildungsgrad bekanntlich grossen Schwankungen unterworfen ist, zeigt jedoch eine erwähmenswerte Besonderheit: die Dornen sind in der 'Tiefe fast ausnahmslos

Grösse als in dem borealen Gebicte». Daraus folgt uur, dass die borealen Indiriduen nio so gross werden wie dic qrüssten arktischen, z. B. die grössten in der Tiefe des Eisfjords, nicht aber, dass sie kleiner sind als z. B. die oberhalb ron $100 \mathrm{~m}$ lehenden Tiere des Fisfords. Wahrseheinlich ist kein oder nur cin unbedeutender Unterschied gegenüber den letzteren vorhanden; G. O. Sırs (1869) gibt füx cin im Kristianiafjord gefundenes Lxemplar ciue Lange von $63 \mathrm{~mm}$ an. 
mehr oder weniger zahlreich, während in seichterem Wasser eine grössere Variabilität vorhanden ist. Bei einer Anzahl aufs geradewohl gewählten Weibchen aus Tiefen zwi-

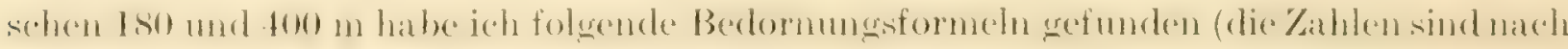
der Grösse der lixemplare geordnet): St. 4 (61 mm Länge): $\frac{5}{4} ;$ St. $42(78-32 \mathrm{~mm}): \frac{6}{4}$, 3. $\frac{7}{4}, \frac{7}{4}$; St. $33(69-40 \mathrm{~mm}): \frac{7}{4}, \frac{7}{4}, \frac{8}{7}(!), \frac{8}{5}, \frac{6}{4}, \frac{6}{4}, \frac{7}{5}, \frac{7}{3}, \frac{7}{2} ; \mathrm{St} .41(78-50 \mathrm{~mm}): \frac{6}{7}(!), \frac{7}{4}$,

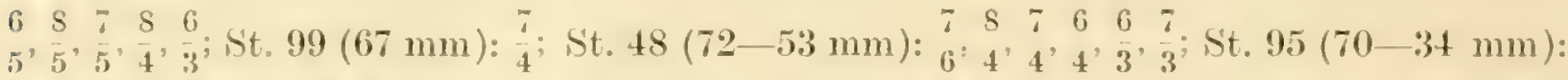
s

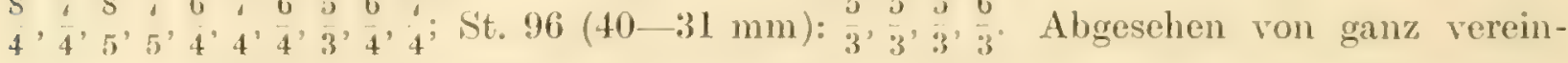
zelten, abweichenden Individuen (oben mit bezeidneet), kam man also folgende allgemeine Bedornungsformel aufstellen: $\frac{(5-) 6-7(-8)}{(3-)+-5(-6)}$. Kleinere Exemplare von 31-60 mm Länge haben entweder dieselbe Anzahl oder bei sehr geringer Grösse bisweilen (St. 96) eine etwas geringere Zahl von Dornen. Bei den Exemplaren aus seichterem Wasser (weniger als $100 \mathrm{~m}$ ) findet man grössere Schwankungen; von grossem Interesse ist dabei die 'Tatsache, dass an derselben Stelle gesammelte Exemplare eine ähnliche Bedormmg aufweisen. An eingen stationen besitzen alle Exemplare nur wenige Dome auf dem Rostrum: St. 73, Advent Bay (48-31 mm Länge): $\frac{4}{2}, \frac{6}{3}, \frac{5}{2}, \frac{4}{3}, \frac{4}{2}, \frac{5}{3}, \frac{5}{3} ;$; St. 61 , Green Bay (55 . 28 mm): $\frac{4}{2}, \frac{4}{2}, \frac{4}{3}, \frac{4}{3}, \frac{5}{2}, \frac{4}{2}, \frac{5}{2}, \frac{5}{2}, \frac{5}{2}, \frac{5}{2}, \frac{4}{4}$; St. 130 , Green Bay (58-44 mm): $\frac{6}{3}, \frac{4}{3}, \frac{4}{2}, \frac{5}{2}, \frac{4}{3}$; St. 83, Billen Bay $(5 \pm-51 \mathrm{~mm}) ; \frac{5}{2}, \frac{4}{3}$. Der allgemeine Bedornungsformel ist also hier $\frac{4-5(-6)}{2-3(-4)^{\circ}}$ An andern Stationen aus denselben Tiefen sind die Dornen ebenso zahlreich wie bei den grosien Weibchen der Fjorltiefe: Nt. 93. Ekman Bay $(54 \mathrm{~mm}): \frac{8}{4} ;$ St. 116, Nordarm $(53-39 \mathrm{~mm}): \frac{6}{4}, \frac{6}{5}, \frac{8}{4}, \frac{7}{3}, \frac{5}{4}, \frac{10}{5}, \frac{7}{5}, \frac{6}{4}, \frac{5}{2}$; St. 79 , Billen Bay $(55-40 \mathrm{~mm}) ; \frac{9}{6}, \frac{7}{4}, \frac{8}{5}, \frac{7}{4}, \frac{7}{3}, \frac{7}{5}, \frac{7}{4}, \frac{5}{4}$; St. 49 , Sassen Bay $(44-37 \mathrm{~mm}): \frac{8}{6}, \frac{6}{3}, \frac{8}{3}$. Wenn man ('ine allgemeine formel für die Bedormung dieser T'iere aufstellen will, bekommt sic das folgende Aussehen: $\frac{(5-) 6-8(-10)}{(2-) 3-5(-6)}$; die Schwankungen sind also etwas grösser als bei den T'Tiefentieren.

Jeder Versuch, tiefer in diese Verhältnisse einzudringen, scheitert an der Spärlichkeit des Materials; deshalb habe ich auch keine anderen Merkmale in dieser Weise untersucht. Jedenfalls liegt der Gedanke nahe, dass mehrere liassen im Eisfjord existieren, die Unterschiede mögen durchwegs nichterbliche, durch die Verschiedenheit der äusseren Bedingungen hervorgerufene Modifikationen oder sie mögen teilweise erblich sein. Vielleicht finden sich eine einheitliche, auch durch bedentendere Grösse gekennzeichnete 'Tiefenrasse und mehrere kleinere Seichtwasserrassen. An zwei mweit von einander gelegenen Stellen in derselben Bai (St. 79 und 83 in der Billen Bay) war jedoch die Bedornung ganz verschieden. Wine Untersuchung dieser Verhältnisse in einem leichter zugänglichen Gebiet, z. B. in den nordnorwegischen Fjorden, würde vielleicht interessante Lrgebnisse liefern. 
Die Temperaturen, unter welchen $S$. polaris im Eisford gefunden wurde, schwankten zwischen $-1,67^{\circ}$ und etwa $+3,7^{\circ}$. Aus unseren Beobachtungen kann man zuerst den Schluss ziehen, dass sie zwischen - $\mathrm{I}^{\circ}$ und $+3^{\circ}$ sehr häufig ist (s. die nachstehende Übersicht). In dem kältesten Wasser $\left(-1,3^{\circ}--1,8^{\circ}\right)$ fanden wir sie nur einmal; diese Seltenheit beruht jedoch zweifellos nur darauf, dass der Grund an den meisten kältesten Stellen aus losem Schlamm bestand. Verwickelter liegen die Dinge in bezug auf die obere Temperaturgrenze. In Wasser von höherer Temperatur als etwa + 3 fehlt die Art so gut wie ganz; sie wurde in solchem nur ein-oder höchstens (St. 25, wo die Temperatur nicht gemessen wurde) zweimal, in dem wärmsten Wasser $\left(+4-5,5^{\circ}\right)$ überhaupt nicht gefangen. Diese, in den beiden oberen Reihen des Schemas zusammengestellten Stationen liegen jedoch in seichtem Wasser; ich muss daher die Frage zu beantworten suchen, ob die hohe Temperatur oder andere mit der geringen Tiefe zusammenhängende Umstände das Tier von der warmen Uferzone des Fjords fernhalten.

\begin{tabular}{|c|c|c|c|c|c|c|c|c|c|c|c|c|c|c|c|c|c|c|c|c|}
\hline$+4,3-+5,6^{\circ}$ & 24 & 28 & 32 & 38 & 39 & 66 & 67 & 70 & 74 & 75 & 76 & 77 & 84 & & & & & & & \\
\hline$+3-+4,3^{\circ}$ & 91 & 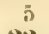 & 7 & 9 & 50 & 54 & 56 & ริ7 & 72 & 85 & 89 & 108 & 111 & 112 & 115 & 121 & 123 & 127 & 128 & \\
\hline$-+3^{2}$ & 4 & $3: 3$ & 41 & 40 & 43 & 51 & $7: 3$ & 92 & 96 & 117 & $126 ;$ & 18 & 19 & 30 & 36 & 49 & 71 & 97 & 106 & $110 \quad 124$ \\
\hline$-+1,0^{\circ}$ & 13 & $\because 6$ & 45 & (6) & 7!) & 80 & $\$ 1$ & S:3 & 86 & 93 & 104 & 116 & 37 & 45 & 87 & 109 & & & & \\
\hline $0-+0.8^{\circ}$ & 41 & 95 & 94 & 102 & 12 & 14 & 16 & 17 & 47 & 103 & & & & & & & & & & \\
\hline $0,0-0,1^{\circ}$ & \pm 1 & 75 & $5: 2$ & 91 & is & 15 & 20 & 22 & 23 & 34 & 35 & 53 & 107 & 113 & 114 & 122 & & & & \\
\hline $1,8--1,5^{\circ}$ & 101 & 54 & 55 & 88 & 100 & 105 & 120 & 125 & & & & & & & & & & & & \\
\hline
\end{tabular}

Die Bodenbeschaffenheit war wenigstens an vielen der warmen Stationen derart. dass das Fehlen nicht darauf beruhen kann. Obgleich unsere Beobachtungen keinen Zweifel übrig lassen, dass S. polaris im Eisfjord nur ganz ausnahmsweise in den mehr als $+3^{\circ}$ warmen Oberflächenschichten lebt, wäre es natürlich wichtig zu konstatieren, ob die Verhältnisse überall ähnlich liegen. Das dürfte wenigstens äusserst wahrscheinlich sein; in Westspitzbergen und andern Gegenden mit ähnlichen hydrographischen Bedingungen ist die Art stets nur in grösserer Tiefe als $20 \mathrm{~m}$ gefunden worden; an der Westkiuste von Grönland ist sie zwar einigemal in geringerer Tiefe gefunden worden, fast ausschliesslich jedoch im nördlichen Teil.

In hocharktischen Gegeden lebt $S$. polaris dagegen häufig in 5-20 m Tiefe. Besonders überzeugend sind die zahlreichen Angaben iiber Vorkommen in seichtem Wasser in den hocharktischen Gegenden von Grönland. Von Nordostgrönland verzeichnen Ohuin, Buchiolz und Hansex 7 Funde zwischen 4 und $20 \mathrm{~m}$, Stephensen (1912) nicht weniger als 24 Funde zwischen 5,5 und $18 \mathrm{~m}$; auf Nordwestgrönland kommen 4 solche Funde (Ortmann 1901). Endlich fand die zweite Framexpedition die Art im Jones Sund an 4 Stationen zwischen 6 und $18 \mathrm{~m} .^{1}$ Hierzu kommen einige Funde in Ost- und Nordwestspitzbergen und an der Westküste von Novaja Semlja, wo das Oberflächenwasser in der Regel nicht so stark erwärmt wird wie im Eisford. Dieser Unterschied in der Vertikalverbreitung wird nur unter der Annahme verständlich, dass die obere Temperaturgrenze in den arktischen Meeren bei etwa $+3^{\circ}$ liegt.

1 SAR9 sagt in seiner Bezrbeitung der Crustaceensammlungen diezer Expedition (1909) nur, lass S. po'.uris "wras found in great abundance in most of the loealities investigated s. Yach fremdlicher brieflicher Miteilung hatten die Fundstellen folgende Tiefen: $10-15$ Faden, $45 \mathrm{~F}, 8 \mathrm{~m}, 6-20 \mathrm{~F}, 15-20 \mathrm{~F}, 10 \mathrm{~F}, 3-7 \mathrm{~F}, 6 \mathrm{~m}, 2-20 \mathrm{~F}$.

K. Rv. Yet. Akad, Hand, Jamil 5t, Non 7. 
Diese Annahme erscheint vielleicht allzu kiihn, da es sich um eine Art handelt, die noch in einem grossen 'Leil der borealen Region häufig ist, wo sie in wärmerem Wasser gut gedeiht. Eine andere Erlklärung der vertikalen Verbreitung im Eisfjord ist jedoch kaum denkbar. Übrigens liegt wohl nichts Ungereimtes in der Annahme, dass eine Art zwar gewisse 'I'emperaturen berorzugt, damit aber eine hohe Anpassungsfähigkeit verbindet, die es ihr erlaubt, auch urspriinglich gemiedene Bedingungen zu ertragen, nämlich wenn keine anderen zu Gebote stehen. Die Lebensweise in borealen Gegenden scheint mir sogar, anstatt die oben gemachte Annahme auszuschliessen, eher eine Stiitze dafiir zu liefern. Die Art fehlt hier nicht nur in denselben geringen Tiefen wie im Eisfjord, sondern noch bis etwa $100 \mathrm{~m}$, und dieser. Unterschied ist sicher durch die Wassertemperatur bedingt (s. minten im Allgemeinen 'Teil). Diese Tatsache lehrt ja, dass die vertikale Verbreitung durch die Temperaturverhältnisse beeinflusst werden kann.

Fortpf a n z ung. En $\mathrm{t} w \mathrm{ick} l u n g$. Eiertragende Weibchen wurden an 13 Stellen erbentet; iiber ihre Grösse s. oben. Für die Beurteilung der Frage nach der Entwicklungszeit der Eier liegen folgende Daten vor; ich berïksichtige dabei auch die von der Kolthoff-Expedition im Juni 1900 im Eisford gefundenen Exemplare:

\begin{tabular}{|c|c|c|c|}
\hline Station & Datum & $\begin{array}{l}\text { o mit nicht oder } \\
\text { wenig entwickeiten } \\
\text { Eiern. }\end{array}$ & $\begin{array}{l}\text { o mit weit ent. } \\
\text { wickelten Eiern } \\
\text { (mit Augen). }\end{array}$ \\
\hline Lotthoff-Exp. & 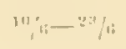 & 2 & 3 \\
\hline 33 & $23 / i$ & 4 & \\
\hline 41 & $24 / 3$ & 3 & 1 \\
\hline 42 & $26 / 7$ & 2 & - \\
\hline 48 & $31 / ;$ & 2 & -- \\
\hline 49 & $31 / \bar{i}$ & 2 & . \\
\hline 61 & $4 / 4$ & $\cdots$ & - \\
\hline 73 & $11 / 4$ & 2 & -- \\
\hline 79 & $13 ; 4$ & 2 & 1 \\
\hline 45 & $31 / 4$ & 2 & - \\
\hline 116 & $25 / 4$ & 3 & 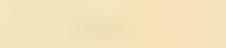 \\
\hline 98 & $27 / 2$ & - & 1 \\
\hline (9) & $27_{i \mathrm{~A}}$ & 1 & $\cdot-$ \\
\hline 130 & $3+1, s$ & 1 & - \\
\hline
\end{tabular}

Man sieht hieraus, dass zahlreiche Weibehen im Sommer Eier tragen, dass aber die Embryonalentwicklung, abgesehen von den frïhesten Stadien, zum grossen Teil erst im Herbst oder Winter vorsichgeht. Doch wurden ja auch im Sommer einige Exemplare mit weit entwickelten Embryonen gefunden. Ob die Entwicklung in diesen Fällen verspätet bzw. verfriiht eingetreten ist oder ob ïberhaupt keine strenge Periodizität vorhanden ist, kann nicht ohne Untersuchungen an einem viel grösseren Material entschieden werden; doch ist es wahrscheinlich, dass die s pät e re Entwicklung in der Regel im Winter stattfindet. HANSEN $(1895,1908)$ zieht aus der Tatsache, dass bei Ostgrönland eiertragende Weibchen von August bis April gefangen worden sind, den Schluss, dass S. polaris „wenigstens an dieser sehr kalten Lokalität" (Hekla Harbour) keine bestimmte Fortpflanzungsperiode hat, sondern sich das ganze oder fast das ganze Jahr hindurch vermehrt. Diese Ansicht wäre jedoch erst bewiesen durch den Nachweis, dass verschiedene Entwicklungsstadien durch das ganze Jahr vorhanden sind. 


\section{Allgemeine Verbreitung.}

(Fig. 3.)

$$
\text { Spitzloorgongobiot: }
$$

Wostspitzborgen: Hornsund, Belsund, Eisfjord, Kings Bay und offenes Mecr (SAms 1886, OIILIN 1901, Birula 1907, Dons 1915 und ohen). [Fermer Krörer 1812: Spitzhergen\%.] Nordwestspitzhergen (HoEk 1882, Sars 1886, Doflein 1900, Onlix 1901, Grieg 1909, Doxs 1915). Nordspitzbergon, zahl. reiche Fundorte (Roos 1828, Dofleis 1900, OnLIx 1901, GRIEg 1909). Os ts pit a be r g en: Ostliüste vom Nordostland, Hinlopenstrasse, K. Karls Land, Storfjord sowic O. und S. davon (MIERs 1877, DofLeIY 1900, STEBBIxG 1900, OHlin 1901, Brrula 1907, Dons 1915). B c o r c ll Eila ll d, sowio N. und No. devon (Sars 1886, DoF LEIN 1900).

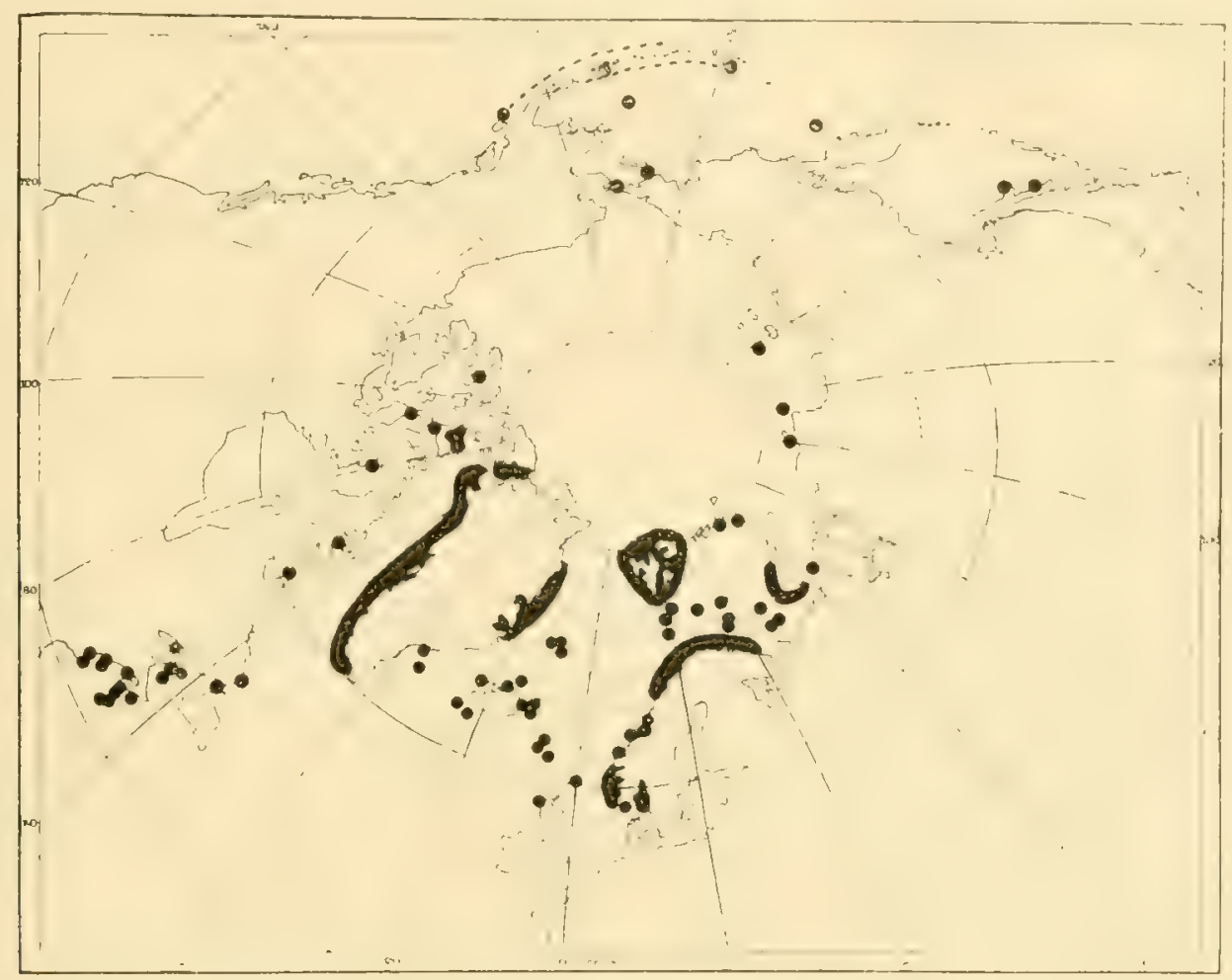

Fig. 3. Spirontocaris polaris.

Die Verbreitung von S. poluris im Spitzbergengebiet würde nach DoFLET (1900, S. 334, 357) diesclbe Be. sonderheit aufweisen, dio er für die ganze Gattung nachweiscn zu können glaubh, und diesor Fall wird besonders oingehend besprochen. Bei Betrachtung der Fänge der deutschen Expeditionen 1898 wällt cs selnr auf, dass 11 . polaris in Nord- und Ostspitzbergen sehr lıäufig ist, während sie in Westspitzbergen fast zu fehlen scheint». Sio wurde in der letzteren Gezend nur an zwei Stellen (im Eisfjord) min nicht geringen 'liefen 》gefunden und wird infolgedesson als ygezen warmes Wasser sehr enpindlielh pezeichnet. Dofterv vergisst hier ganz, dass dio Art an der skandinavischen Küste allgomein ist. Nach unse:en Beobachtungen ist sic ja ausserordentlich häufig im lisfjord, und dio Empfindlichkeit gegen warmes Wasser beginnt erst bei etwa + 30; dis Art muss also stets in Westspitzbergen günstige Lebensbedingungen finden. Dass dies anch im Sominer 1898 der Fall war, wird ausser durch dio Beobachtungen der deutschen Expeditionen durch mehrere Funde der sehwedischen Nathorst-lixpedition orwieson (s. oben.)

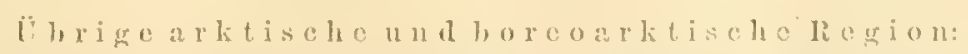

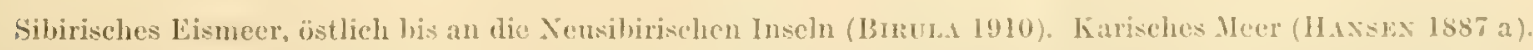

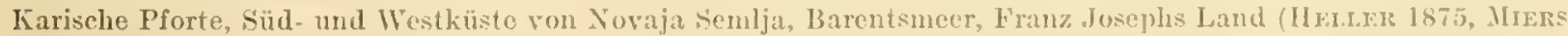

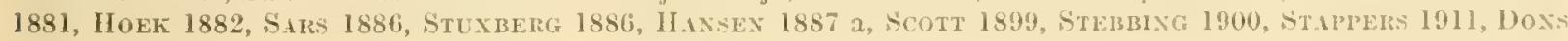
1915). Weisses Meer (keino Lokalangaben, nicht auf der Karte Fig. 3 herücksichtigt). Murmanküste (JAkzrasir 


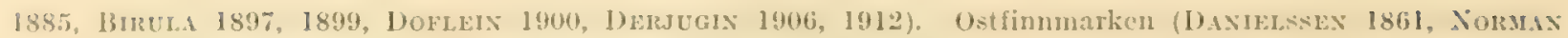
1894, 1902, NormaAard 1905, Doss 1915; fermer M. S.13s 1858: Vinmmarken). Kalte Fjorde in Westfinnmarken

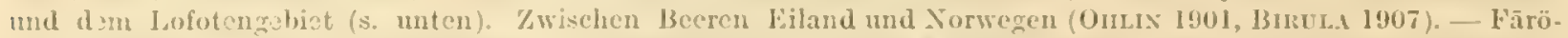

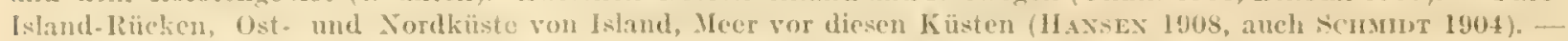

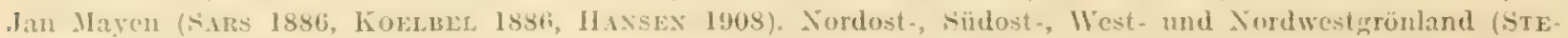

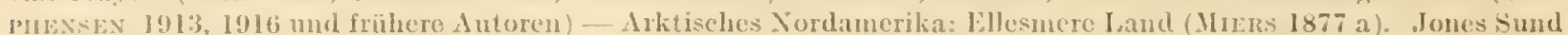

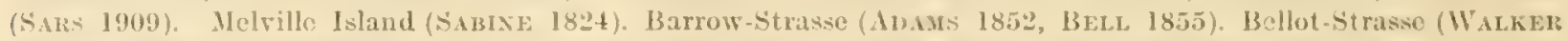
1860). Melville-Halbinsel (Iglootik Island) (Ross 1835). Cumberland Sund (Y'FEFFE 1886 [1/ipp. 1mazo]). IIndon-Strasse, östlicher Eingang (Wnidivis 1901). - Nordküsto von Labrador, Ciolf von St. Lawrenec,

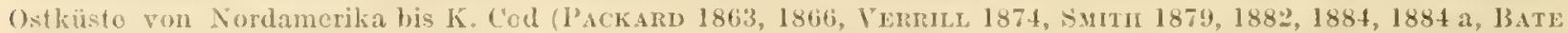

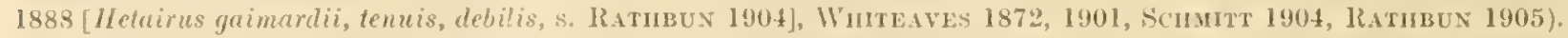

l'azifisches Gobist: Mrktischer Ozcan म ( $\mathrm{N}$. vou der Beringsstrasse) (BTmpsox 1860). Beringsstrasse, 13eringsmecr, Aleuten, Bering Island (RATHuUx 1904, auch BRaxdt 1851 [Hip). St. I'ati]). Ochotskisches Meer (Brasısukow 1907).

$$
13 \text { orcale } \mathrm{R} \text { ○ g i o 11: }
$$

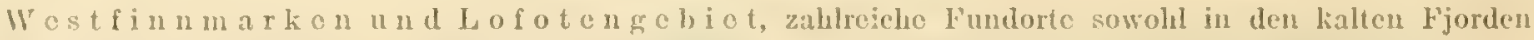
wis in dor warmen Aroa (II. SARS 1858, DANILLSEN 1861, G. O. S.11S 1886, SPAMTE-SCINIEIDE 1884, AURIVILIU. 1886, Norua 1894, 1902, Krer 1906 a, besonders Nordgativ 1905, Doxs 1915). Nor w c is c he lV os t. $\mathrm{k}$ ï s to südlich davon: Helgeland (Doss 1915). Trondhjemsfjord (STorm 1878, 1880, Norm.x 189t). Kristian-

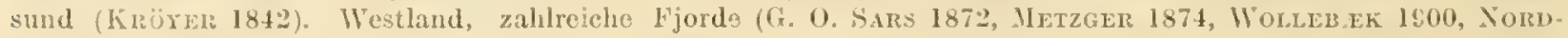

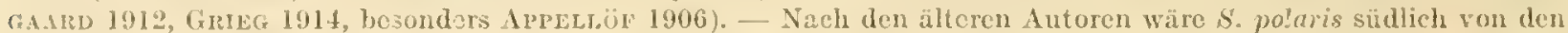
Jofoten selten (M. S.IRs, KrörER; dex letztero kannto fon dieser ganzen Küstenstreck nur zwei Lxenplare, beide hei Kristiansund gefangen). Schon G. O. S.Ars (1872) und Śtora fanden jedoch, dass die Art im 11ardanger-und im Trondhjomsford häufig ist; spâtor hat es sich gezoigt, dass sie meino unscrer häufigsten Hippo'yte-Artcn in den grösseran Ijord'iefen ist (Appeldör). Die scheinbaro Seltenheit beruht daranf, dass sio hier nur in grosser Tisfe zu findon ist.

Sk a y rak: Norworischo Küste: Vor Mandal (Metzger 1874). Breviksjord, Kristianiafjord (C. O.

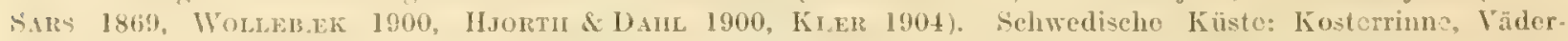

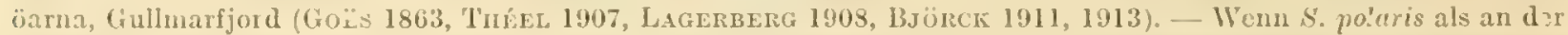
Küsto von Bohuslän selten bezoichnot werlen muss (LAGerBerG), so ist dio Seltenheit zweifellos hauptsāehlich oino Folve dor Tiefenverhaltnisse; in dor tiefen Kosterrino ist dio Art nach Bsörck nieht besonders selten. Im Kattegat, wo dio Tiofo nirgends über 100, meistens nicht über $50 \mathrm{~m}$ beträgt, fohlt sio ganz. Yon Interesse ist, dass sio auf der schwedischen Seite des offenen skageraks zu fehlen scheint (BJörck); vor der norwegischen İ üsto dirfte sio nur sporadisch auftreten (der oben erwähute Fund por Mandal).

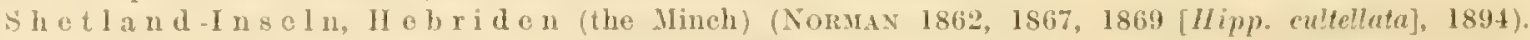

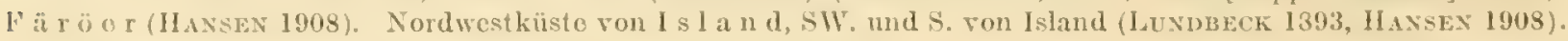

Bathymetrische Verbreitung. In der arktischen Region ist die bathymetrische Verbreitung sehr bedeutend; sie umfasst die 'Tiefen von 3 oder 5 bis nahezu $1000 \mathrm{~m}$ (478-553 Faden; HANSEx 1908). In der Uferzone, oberhalb von 20 oder $25 \mathrm{~m}$, lebt die Art jedoch, wie ich oben nachgewiesen habe, nur in hocharktischen Gegenden. Unterhalb von $650 \mathrm{~m}$ ist sie bisher nur dreimal gefangen worden (SARS 1886, HANSEN 1908); die meisten Funde stammen aus geringerer Tiefe als $300 \mathrm{~m}$; doch ist es möglich, dass das Tier noch beträchtlich weiter abwärts ebenso häufig ist. In der b orea 1 en Region lebt die Art, wie ApPextöF hervorhebt, in der Regel nur unterhalb von $100 \mathrm{~m}$ (die zahlreichen Fundorte, welche der genannte Autor verzeichnet, liegen alle in Tiefen zwischen etwa 100 - der oberste 75 bis 95 - und $550 \mathrm{~m}$ ).

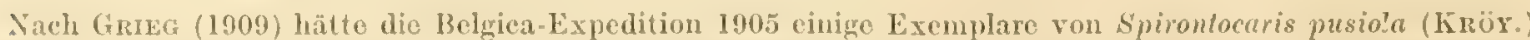
im Eisfjord (Green Bay) gefunden. Da dio erwähnte Species oine boreale Art ist, die zwar in die boreoarktische, nicht aber in die arktische Region eindringt(s. z. B. DOFLeiv 1900, Appectör 1906), müsste dieser Fund bemerkenswert arseheinen, un so mehr als in unserem grossen Material kein cinziges Exemplar ron s. pusiola vorhanden ist. Durch freundliches Iintgegenkommen von Dr. E. KoErow, Bergen, hahe ich das Material untersuchen können ma d ahei gefunden, dass es sich um cinen Irrtum handelt; dio als S. pusio? bestimmten Exemplaro sind junge, aber ganz typische Exemplare von S. polaris. S. pusiola ist folglich aus der Fauna des Lisfjords zu streichen. 
Dass S. pusio? als zufäliger (iast in der arktischen Region auftreten könnte, ist natïrlich trotzdem nicht ausgeschlossen. Nach DOxLEIN (1900) wurdo sio in der Tat von der Helgoland-Lxpedition in Spitzibergengebiet gefunden, und zwar auffallenderwoiso in Ostspitzbergen (IIalbmondinsel), also unter zicmlich ausgesprochen hoch. arktischen Bedingungen. Da man hier noch weniger als im Eisford das Vorkommon dieser Art erwarten könnto, muss man sich fragen, ob nicht auch in dissem Falle cin Irrtum möglich ist.

\title{
Spirontocaris gaimardii (H. Mune-Edwards).
}

\author{
Syn. S. gibba Krör. \\ S. belcheri (BELL) (s. unten).
}

Die S. gaimardii des Eisfjords gehört zu der für die meisten arktischen, aber nicht hocharktischen Gegenden charakteristischen Rasse. Die Weibchen entbehren stets der stachelartigen Verdickung auf der Rückenseite des dritten Abdominalsegmentes. Die Männchen besitzen. von einer gewissen Grösse an alle einen solchen Stachel; dieser ist in der Regel schon bei einer Körperlänge von 33 oder $34 \mathrm{~mm}$ angedeutet, bei 38 bis $40 \mathrm{~mm}$ Länge ist er deutlich, bei $50 \mathrm{~mm}$ stark entwickelt.

BIRUlA hat neuerdings (1910) den Versuch gemacht, diese Art in drei Rassen zu zerlegen: 1. S. gaimardii gaimardii (MILNE EDWARDS): eine boreale Rasse; 2. S. gaimardii gibba (KR.) (Dons [1915] nennt sie »forma typica»): eine Zwischenform mnicht nur geographisch sondern auch morphologisch », hauptsächlich in den Gewässern Spitzbergens verbreitet; 3. S. gaimardii belcheri (BELL.): östliche Rasse; diese letztere Form wird eingehend beschrieben. BiruLA's Untersuchungen liefern einen wichtigen Beitrag zur Kenntnis der Morphologie und Variabilität dieser Art, doch haben sie nicht den Beweis dafür geliefert, dass die drei Rassen wirkliche geographische Subspecies darstellen. Es könnte sich ebensogut um nicht-erbliche Modifikationen handeln; vieles spricht sogar, soweit ich sehen kann, entschieden für die Möglichkeit, dass die Unterschiede teilweise diese Bedeutung haben. Besonders wichtig ist in dieser Beziehung eine Tatsache. S. gaimardii belcheri - gekennzeichnet vor allem durch das Vorkommen eines Abdominalvorsprunges auch beim weiblichen Geschlechte - ist nach BIRULA eine östliche Rasse, die vom östlichen Teil des Barentsmeeres bis zum Nordamerikanischen Archipel verbreitet ist. Er bemerkt aber selbst, dass belcheri-ähnliche Exemplare auch in Westgrönland (B. schreibt irrtümlich Ostgrönland), bei Labrador und, nach seinen eigenen Beobachtungen, bei Nordspitzbergen gefunden worden sind. Diese Rasse ist also z i $\mathrm{k} \mathrm{u} \mathrm{m} \mathrm{p}$ o $\mathrm{l}$ a $\mathrm{r}$ : sie ist ferner hocharktisch oder mit andern Worten: in hocharktischen Gegenden erhält die Art S. gaimardii, wenigstens unter gewissen Bedingungen (vorwiegend im Gebiet Novaja Semlja -Nordamerikanischer Archipel?), belcheri-Merkmale.

S. gaimardii gibba, bei der u. a. der Abdominalvorsprung nur im männlichen Gesehlecht vorhanden ist, lebt von Westgrönland bis Novaja-Semlja unter niederarktischen oder boreoarktischen, bisweilen auch unter hocharktischen Bedingungen; vom pazifischen Gebiet erwähnt Ratmbun nur S. gaimardii belcheri, doch ist es wohl möglich, dass unter gewissen Bedingungen die gibba-Form oder gar die typische Form anch hier entstehen kann (R. erwähnt Exemplare, wwhich unite the characters with those of S. gaimardi》). 
Hieraus scheint hervorzugehen, dass S. gaimardii je nach den physikalischen Bedingungen die Merkmale der ersten, zweiten oder dritten Rasse erhält. Es ist sehr wohl möglich, dass die Unterschiede teilweise erblich fixiert sind oder dass ein und dasselbe Merkmal in einer Gegend eine nicht-erbliche Modifikation, in einer andern erblich ist, dariiber ist aber nicht das Geringste bekannt. Vielleicht hat der russische Forscher im Grunde dieselbe Auffassung; er hebt selbst hervor, dass er keine konstanten Merkmale auffinden konnte und deshalb die drei formen nur als „Unterarten resp. geographische Rassen einer und derselben circumpolaren boreoarktischen Art» betrachtet. Wenn aber die Verbreitungsgebiete nicht getrennt sind und keine konstanten Unterschiede vorkommen, darf man auch nicht von geographischen Unterarten reden.

\section{Verbreitung im Lisfjord.}

Verzeichnis der Fundorte (s. Karte 4):

\begin{tabular}{|c|c|c|c|c|c|c|c|c|c|c|}
\hline $\begin{array}{l}\text { Nr. } \\
\text { der } \\
\text { Stat. }\end{array}$ & Ort und & A Da & atum & & Tiefo & $\begin{array}{l}\text { Wasser- } \\
\text { temperatur }\end{array}$ & $\begin{array}{l}\text { Salz- } \\
\text { gelialt } \\
0 ; u\end{array}$ & Bodenbeschaffenhoit & Gerït & $\begin{array}{l}\text { Anzialil und } \\
\text { Grösso }\end{array}$ \\
\hline 13 & $\begin{array}{l}\text { Eingang in } \\
\text { Bay . . }\end{array}$ & dio & Saf & .16 .7 & $125-150 \mathrm{~m}$ & $144 \mathrm{~m}:+1,23^{\circ} \mathrm{Ir}$ & 34,54 & $\begin{array}{l}\text { Schlamm mit Schnlen; } \\
\text { Balanus porcalus-Ge- } \\
\text { moinschaft }\end{array}$ & Trawl & $\begin{array}{l}27 \text { Fx. } 43- \\
59 \mathrm{~mm}(3 \text { \% } \\
\text { ovig. })\end{array}$ \\
\hline 5 & Safo Bay & - & - . & .15 .7 & $2-8 \mathrm{~m}$ & {$\left[+3,3 \text { bis }+4^{\circ}\right]^{14}$} & - & $\begin{array}{l}\text { Stoin und IKies mit La. } \\
\text { minarien (und oin werig } \\
\text { Schlamm) }\end{array}$ & Kl. Dredgo & $\begin{array}{l}3 \text { Ex., 19- } \\
37 \mathrm{~mm}\end{array}$ \\
\hline 6 & · & . $\cdot$ & • $\cdot$. & - $\quad x$ & $40 \mathrm{~m}$ & - & $\cdots$ & Schlamm, otwas Stcin | & $"$ & $2 \mathrm{Ex}, 36 \mathrm{~mm}$ \\
\hline 8 & » & . & * & . & $35 \mathrm{~m}$ & - & 一 & Fester Schlanum & " & $1 \mathrm{Ex}, 32 \mathrm{~mm}$ \\
\hline 12 & $" \quad$ " . & . & . & .167 & $118-127 \mathrm{~m}$ & $108 m:+0,65^{\circ}$ & 34,43 & Loser Schlamm & Trawl & 1 Ex. $41 \mathrm{~mm}$ \\
\hline 20 & Ymer Bay. & . & . . & 20.7 & $85-100$ II & $85 \mathrm{~m}:-0,2 \mathrm{~s}^{\circ}$ & 34,54 & $\begin{array}{l}\text { Loser Schlamm, stellon. } \\
\text { woise Stein mit Algen }\end{array}$ & 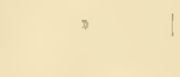 & $1 \mathrm{Ex}, 47 \mathrm{~mm}$ \\
\hline 26 & w & $\cdot$ & $\cdot$ & $\cdot{ }^{\prime}$ & $78-50 \mathrm{~min}$ & $75 \mathrm{~m}:+1,7^{\circ}$ & - & $\begin{array}{l}\text { Fester und züher } \\
\text { Schlamm }\end{array}$ & KI. Dredgo & $\begin{array}{l}6 \text { Ex., } 38- \\
62 \mathrm{~mm}(1+ \\
\text { ovig. }\end{array}$ \\
\hline 27 & $"$ & . . & . $\cdot$ &. & $30 \mathrm{~m}$ & - & 一 & $\begin{array}{l}\text { Fies und Stein mit Litho- } \\
\text { thamnion Krusten mnd } \\
\text { Balanus porcatus. }\end{array}$ & » & I Ex., $34 \mathrm{~mm}$ \\
\hline 30 & $"$. & .. & - $\cdot$ & .21 .7 & $9-5 \mathrm{~m}$ & {$\left[+2 \text { bis }+2,5^{\circ}\right]^{\mathrm{v} 1}$} & - & $\begin{array}{l}\text { Zither Schlamm mit Sto:- } \\
\text { nen und Laminarien. } \\
\text { resten }\end{array}$ & $"$ & $1 \mathrm{Ex}, 47 \mathrm{~mm}$ \\
\hline 33 & Fjordstamm & . . & . & .23 .7 & $263-25611$ & {$\left[+2 \text { bis }+2,6^{\circ}\right]^{\mathrm{vur}}$} & - & Loser Schlanm & Trawl & $\begin{array}{l}4 \text { Ex., } 38- \\
54 \text { mm }(1 \text { o } \\
\text { ovig. })\end{array}$ \\
\hline 11 & " & . $\cdot$ & . . & .24 .7 & $234-254 \mathrm{~m}$ & $251 \mathrm{s1}:+2,50^{\circ}$ & 34,96 & s & » & $1 \mathrm{Ex}, 45 \mathrm{~mm}$ \\
\hline 21 & $\begin{array}{l}\text { Eingang in d } \\
\text { Bay . . }\end{array}$ & lio $\mathrm{T}$ & und & .20 .7 & $71-68 \mathrm{~m}$ & $-0,93^{\circ}$ & 34,29 & $\begin{array}{l}\text { Loser Schlumm, stellon-! } \\
\text { weise Stein }\end{array}$ & $"$ & $\begin{array}{l}0 \text { Lx., } 44- \\
56 \mathrm{~mm}(1 \text { 우 } \\
\text { ovig. })\end{array}$ \\
\hline 94 & Fjordstairm & . . & . . & .21 .8 & $147-141 \mathrm{~m}$ & $140 \mathrm{~m}:-0,62^{\circ}$ & 34,49 & $\begin{array}{l}\text { Loser Schlamm mit klei- } \\
\text { nen Stoinen }\end{array}$ & . & $\begin{array}{l}15 \mathrm{Ex}, 34- \\
65 \mathrm{~mm}(3 \% \\
\text { ovig. }\end{array}$ \\
\hline 92 & Nordarm & . & • . & .19 .8 & $85-45 \mathrm{~m}$ & $42 \mathrm{~m}:+2,02^{\circ}$ & 一 & $\begin{array}{l}\text { Loser Schlamm mit Kies } \\
\text { und Sand. Am Finde } \\
\text { der Dredgung Steino } \\
\text { und Laminarien }\end{array}$ & ” & $\begin{array}{l}34 \text { Fx. } 33- \\
62 \mathrm{~mm}(5 q \\
\text { ovig. })\end{array}$ \\
\hline 50 & $\begin{array}{l}\text { Nordarm, Wi } \\
\text { dio Yoldia }\end{array}$ & $\begin{array}{l}\text { inga: } \\
13 a\end{array}$ & $\begin{array}{l}\operatorname{lng} \text { is } \\
\text { ay }\end{array}$ & 19.8 & $17--60 \mathrm{~m}$ & - & - & $\begin{array}{l}\text { Zäher Schlamin mit İies } \\
\text { und Sand }\end{array}$ & Iil. Drodgo & $\begin{array}{l}3 \mathrm{Lx}, 45- \\
50 \mathrm{~mm}\end{array}$ \\
\hline
\end{tabular}


KUNGI. SV. VET. AKAIEMIENS IIANIIINGAR. RAND 54 N:07.

\begin{tabular}{|c|c|c|c|c|c|c|c|c|c|c|}
\hline $\begin{array}{l}\text { Nr. } \\
\text { der } \\
\text { Stat. }\end{array}$ & Ort und & Da & tum & & Tiefe & $\begin{array}{l}\text { Wassor- } \\
\text { tomparatur }\end{array}$ & $\begin{array}{l}\text { Sulz. } \\
\text { gohalt } \\
\% \text { : }\end{array}$ & Bodenbeschaffonheit & Gerät & $\begin{array}{l}\text { Anzahl und } \\
\text { Crösse }\end{array}$ \\
\hline 93 & Ekman Bay & . & - & .208 & $44-5.5 \mathrm{~m}$ & $+1,72^{\circ}$ & - & $\begin{array}{l}\text { Zäher, stark roter } \\
\text { Sichlamm. Etwas Stoin }\end{array}$ & Traw ! & $\begin{array}{l}6 \mathrm{Ex}, 42- \\
03 \mathrm{~mm}(2 \text { f } \\
\text { ovig. })\end{array}$ \\
\hline 117 & $\begin{array}{l}\text { Fingang in d } \\
\text { son Buy . }\end{array}$ & die & & .25 .8 & $29-27 \mathrm{~m}$ & {$\left[\operatorname{etwa}+2^{\circ}\right]^{x}$} & - & $\begin{array}{l}\text { Strauchförmiges Litho- } \\
\text { thamnion auf Schlamm- } \\
\text { boden }\end{array}$ & Iil. Dredgo & $1 \mathrm{Ex}, 39 \mathrm{~mm}$ \\
\hline 120 & Dickson Bay & . . & $\cdot$ & .27 .8 & $98 \mathrm{~m}$ & $93 \mathrm{~m}:-1,63^{\circ}$ & 34,27 & Loser Schiamm & Trawl & $\begin{array}{l}3 \mathrm{Ex}, 37- \\
52 \mathrm{~mm}\end{array}$ \\
\hline 121 & $\begin{array}{l}\text { Eingang in d } \\
\text { son Bay. }\end{array}$ & lio $\mathrm{D}$ & & -208 & $5 \mathrm{~m}$ & {$\left[+3,7^{\circ}\right]^{\mathrm{x}}$} & - & $\begin{array}{l}\text { Schlamm mit Kies, Schn. } \\
\text { len und Steinen }\end{array}$ & İ́l. Dredgo & $1 \mathrm{Ex}, 44 \mathrm{~mm}$ \\
\hline 78 & Billen Bay & . & & .13 .8 & $113-116 \mathrm{~m}$ & {$\left[0 \text { bis }-1^{\circ}\right]^{X I V}$} & - & Loser Schlamm & s & $1 \mathrm{Fx} ., 44 \mathrm{~mm}$ \\
\hline 79 & » & . & &.$\infty 1$ & $32-40 \mathrm{~m}$ & {$\left[+1,5\right.$ bis $\left.+2^{\circ}\right] \mathrm{xv}$} & - & $\begin{array}{l}\text { Steine mit strauchför- } \\
\text { migem Lithothamnion }\end{array}$ & $n$ & $\begin{array}{l}8 \text { Fx. } 38- \\
57 \mathrm{~mm}(2 \text { 운 } \\
\text { ovig. })\end{array}$ \\
\hline 80 & $\begin{array}{l}\text { Eingang in di } \\
\text { Bay. }\end{array}$ & io $13 i$ & & .14 .8 & $69-6+m$ & $69 \mathrm{~m}:+1,5^{\circ}$ & - & $\begin{array}{l}\text { Luser Schlonm (mit oin } \\
\text { wenig Sand und Kies) }\end{array}$ & ? & $\begin{array}{l}3 \mathrm{Ex}, 35,38 \\
55 \mathrm{~mm}(1 \text { ? }\end{array}$ \\
\hline 81 & $\begin{array}{l}\text { Eingang in di } \\
\text { Bay }\end{array}$ & io $\mathrm{Bi}$ & iller & .14 .8 & $26 \mathrm{~m}$ & $+1,83^{\circ} \times v I$ & 33,77 & $\begin{array}{l}\text { Stranchförmiges Lilho- } \\
\text { thamnion, etwas Kies }\end{array}$ & 8 & $\begin{array}{l}\text { ovig.) } \\
1 \mathrm{Ex}, 32 \mathrm{~mm}\end{array}$ \\
\hline 82 & Billen Bay . & . & $\cdot$ & .15 .8 & $65 \mathrm{~m}$ & $-0,7^{\circ}$ & - & $\begin{array}{l}\text { Teils loser Schlamm, teils } \\
\text { fester Schlamm mit } \\
\text { Steinen und IKeis }\end{array}$ & $v$ & $\begin{array}{l}10 \mathrm{Ex}_{4} 35- \\
52 \mathrm{~mm}\end{array}$ \\
\hline 83 & $" \quad \cdot$ & . . & & .16 .8 & $22 \mathrm{~m}$ & {$\left[\right.$ etwa $\left.+1, s^{\circ}\right] \times$ rII } & - & $\begin{array}{l}\text { Sndgemischter, fester } \\
\text { Schlanm mit Kies und } \\
\text { Stcinen }\end{array}$ & 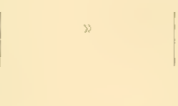 & $\begin{array}{l}7 \text { Ex., 29- } \\
38 \mathrm{~mm}\end{array}$ \\
\hline 86 & , & . : & - & . $"$ & $30 \mathrm{~m}$ & $+1,6^{\circ}$ & - & Kies und lileine Stoine & » & $\underset{\mathrm{mm}}{2 \mathrm{Ex}}, 38,51$ \\
\hline 101 & $\times \quad$ & . . & . & .148 & $150-140 \mathrm{~m}$ & $140 \mathrm{~m}:-1,67^{\circ}$ & 34,43 & $\begin{array}{l}\text { Loser Schlamm mit Stei- } \\
\text { nen }\end{array}$ & Trawl & $\begin{array}{l}23 \mathrm{Ex}, 29-1 \\
55 \mathrm{~mm}(4 \text { q } \\
\text { ovig. })\end{array}$ \\
\hline 87 & ? & . . & & .178 & $37-35 \mathrm{~m}$ & $+1,5^{\circ}$ & - & $\begin{array}{l}\text { Sehr losor Sclulamm, otwas } \\
\text { Kies }\end{array}$ & IKl. Dredge & $\underset{\mathrm{mm}}{2 \mathrm{Ex}}, 38,42$ \\
\hline 49 & Sassen Bay, & Ban & & .31 .7 & $\begin{array}{l}24-19 \text { und } \\
19-28 \mathrm{~m}\end{array}$ & {$\left[+2\right.$ bis $\left.+3^{\circ}\right] \times x x$} & - & $\begin{array}{l}\text { Stein, Kies und Schalen } \\
\text { mit Lithothamnion }\end{array}$ & 'I'rawI & $\begin{array}{l}20 \mathrm{Ex}, 30- \\
42 \mathrm{~mm}\end{array}$ \\
\hline 47 & $\begin{array}{l}\text { Eingang in } \\
\text { Bay }\end{array}$ & die & & $\begin{array}{l}\text { inssen } \\
.29 .7\end{array}$ & $97-120 \mathrm{~m}$ & $\left\{\begin{array}{c}{\left[\text { otwa } 0^{\circ}\right] \mathrm{xdv}} \\
\left(8: 2 \mathrm{~m}:+1,71^{\circ}\right)\end{array} \mid\right.$ & - & Loser Schlamm & Oiterirawl & $\begin{array}{l}7 \text { Fx., } 46- \\
56 \mathrm{~mm}(3 \xi \\
\text { ovig. })\end{array}$ \\
\hline 48 & Ostarm . . & . & & .31 .7 & $199-226 \mathrm{~m}$ & $210 \mathrm{~m}:+1,27^{\circ}$ & 34,72 & ; & Trawl & $6{ }_{63} \mathrm{E} x, 42-$ \\
\hline 104 & Fjordstamm & - & & .17 .8 & $260 \mathrm{~m}$ & $270 \mathrm{~m}:+1,62^{\circ}$ & 34,79 & 2 & " & $\mid \begin{array}{l}2 \text { Fix., 39, } 51 \\
\text { mm }\end{array}$ \\
\hline 44 & $\begin{array}{l}\text { Eingang in } \\
\text { vent Bay }\end{array}$ & dis & & $1-27.7$ & $150-110 \mathrm{~m}$ & $128 \mathrm{m:}+0,01^{\circ}$ & 34,54 & Loser Schlomm mit Isies & s & $\begin{array}{l}30 \mathrm{Ex} ., 35- \\
53 \min (6 q \\
\text { ovig })\end{array}$ \\
\hline 73 & Advent I3ay & . & . & .11 .8 & $35-30 \mathrm{~m}$ & $\begin{array}{l}{[+2 \text { bis }} \\
\left.+2,7^{\circ}\right] \times \times 11\end{array}$ & - & $\begin{array}{l}\text { Balanus porcalus.Ge- } \\
\text { meinscl. IRirs tund } \\
\text { Stein }\end{array}$ & Iil. Dredge & ${ }_{40 \mathrm{~mm}}^{4 \mathrm{Ex}, 2 \mathrm{I}-}$ \\
\hline 95 & Fjordstamm & - & - & .21 .8 & $188-181 \mathrm{~m}$ & 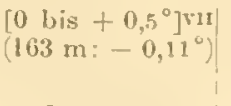 & 34,47 & Sclilamm mit Stoinen & I'rawl & $\begin{array}{l}23 \mathrm{Ex}, \frac{25-}{65 \mathrm{~mm}(5} \\
\text { ovig.) }\end{array}$ \\
\hline 69 & Colos 13.1y . & . & & - 8.8 & il m & $\begin{array}{l}{[+1,5 \text { lis }} \\
\left.+2^{\circ}\right] \times x i v\end{array}$ & - & $\begin{array}{l}\text { Ijos, stcin und Schalen. } \\
\text { Etwas Lithothamnion }\end{array}$ & Ki. Dredge & $\begin{array}{l}4 \text { Lx., } 35 \\
5 \cdot 2 \mathrm{~mm}(1 \mathrm{f} \\
\text { ovig.) }\end{array}$ \\
\hline 126 & Fjordstamm & . & & .30 .8 & $47-31 \mathrm{~m}$ & $\left.\left[+2 b i s+3^{\circ}\right] \times x v\right\}$ & - & $\begin{array}{l}\text { Balanus porcatus-Go- } \\
\text { meinsch. }\end{array}$ & " & $\begin{array}{l}13 \mathrm{Fx}, 25- \\
52 \mathrm{~mm}\end{array}$ \\
\hline 129 & D & & & " & $65 \mathrm{~m}$ & - & - & $\begin{array}{l}\text { Sondgemischter Sohlamm } \\
\text { mit Kieg und modern- } \\
\text { don Algenrosten }\end{array}$ & $=$ & $\begin{array}{l}8 \mathrm{kx}, 30- \\
50 \mathrm{~mm}\end{array}$ \\
\hline
\end{tabular}




\begin{tabular}{|c|c|c|c|c|c|c|c|c|c|c|c|}
\hline $\begin{array}{l}\text { Nr. } \\
\text { der } \\
\text { Stat. }\end{array}$ & Ort & t und & Dat & um & & Tiefo & $\begin{array}{l}\text { Wasser- } \\
\text { ternperatur }\end{array}$ & $\begin{array}{c}\text { Sulz- } \\
\text { gehalt } \\
0 / \text { no }\end{array} \mid$ & Budenbeschaffenheit & Gerät & $\begin{array}{l}\text { Anzahl und } \\
\text { Grösse }\end{array}$ \\
\hline 103 & $\begin{array}{l}\text { Greon I } \\
\text { Eingn }\end{array}$ & $\begin{array}{l}\text { I3ay ns } \\
\text { ung. }\end{array}$ & alio b & seim & .17 .8 & $130 \mathrm{~m}$ & $+0,58^{\circ}$ & - & $\begin{array}{l}\text { Loser Schlamm. Einige } \\
\text { Steino und Balanus } \\
\text { porcalus }\end{array}$ & 'lruwl & 2 Ex., $26 \mathrm{~mm}$ \\
\hline 130 & Creen & Bßny & . . & & .30 .8 & $40-45 \mathrm{dn}$ & $一$ & & $\begin{array}{l}\text { Schilamm mit Algen- } \\
\text { resten }\end{array}$ & Iil. Dredge & $\begin{array}{l}4+\mathrm{kx}, 25- \\
\text { (i0 min }(29 \\
\text { (1vig.) }\end{array}$ \\
\hline $5 !$ & n & " & . . & . . & .38 & Etwr $\$ 10 \mathrm{~m}$ & - & - & $\begin{array}{l}\text { Loser Schlamm mit Tei- } \\
\text { len vo:a Iandpflanzon }\end{array}$ & ” & $\left\{\begin{array}{l}\text { 1i Ex., } 33- \\
39 \mathrm{~mm}\end{array}\right.$ \\
\hline 10 & 。 & $"$ & . & - . & $\cdot \Rightarrow 1$ & $33 \mathrm{~m}$ & - & . & $\begin{array}{l}\text { Kiea, Stein und Schn!en } \\
\text { mit Lithnthamnion-Kilu- } \\
\text { sten; zuhlr. K. porcatus }\end{array}$ & " & $\begin{array}{l}15 \mathrm{Ex}, 332 \\
43 \mathrm{man}\end{array}$ \\
\hline 61 & : & , & . . & . . & 4.8 & $46-35 \mathrm{~m}$ & - & $\cdots$ & $\begin{array}{l}\text { Kies und Stejn. Balanus } \\
\text { porcatus Gemeinsch. }\end{array}$ & ? & $\begin{array}{l}15 \text { E.x., } 33- \\
57 \mathrm{~mm}\end{array}$ \\
\hline 67 & x & $\Rightarrow$ & . & . . & . 6.8 & $2 \mathrm{~m}$ & {$\left[\right.$ [etwa $\left.+s^{\circ}\right]$ xxvir } & - & $\begin{array}{l}\text { Loser Schlamm mit iro- } \\
\text { dernden I'flanzenteilen }\end{array}$ & H. & $1 \mathrm{Fx}, 35 \mathrm{~mm}$ \\
\hline
\end{tabular}

[Ausser in Eisfjord fanden wir S. gaimardii im If o r m s u $\mathrm{d}$ (Gö̈' Bay, 10. 7, 10 his $35 \mathrm{~m}$, Schlamm und Kies; 47 Lix., Lüngo $28-52 \mathrm{~mm})$.]

Frühoro Fundo im Eis f jord:

Sehwed. Lxped. 1858-1868: Sassen Bay, 3,5 m; Adrent Bay, 5,5 his 9, 9 bis 18, 54 his $72 \mathrm{~m}$; Gron Bay, 9 bis 27,9 his $36,36 \mathrm{~m}$; Fisfjord ohne Lokalangabe, 36,45 his $90 \mathrm{~m}$ (Riksmuseum, Stockholm, nach A. MoLANDER).

Miers 1877: Green Bay. Norweg. Fordmeerexped. 1878: Advent Bay, $110 \mathrm{~m}$, +0,7º, Schlamm (SARs 1886). Schwed. Exped. 1898: Fjordstamm, 40 bis $50 \mathrm{~m}$; 1899: Green Bay, $110 \mathrm{~m}$; 1900: Coles Bay, $100,50 \mathrm{llis} 100 \mathrm{~m}$, Stein (OnLIN 1901). Russ. Exped. 1899, 1900: Billen Bay, 142 bis $133 \mathrm{~m},-1,9^{\circ}$, Schlamm mit Stoinen; Fjord. stamm, $243 \mathrm{~m},-0,8^{\circ}$; Advent Bay, 7 bis $9 \mathrm{~m}$, Grus; $9 \mathrm{~m}$, Stein; Green Bay, $30 \mathrm{~m},+3^{\circ}$, schlammiger Sand (BIRULA 1907). Belgica-Exped. 1905: Green Bay (Grieg 1909).

Spirontocaris gaimardii wurde von uns im Eisfjord an 44 Stationen, in zusammen 379 Exemplaren, angetroffen. Sie ist also ungefähr ebenso allgemein wie $S$. polaris, obgleich sie etwas öfter vereinzelt oder in geringer Individuenzahl erbeutet wurde. Wenn man von den vereinzelten Funden oberhalb von $10 \mathrm{~m}$ absieht, wurde sie an $48 \%$ der in den von ihr bewohnten Tiefen untersuchten Stationen gefangen.

Hinsichtlich der $\mathrm{B}$ o de $\mathrm{n}$ b e s $\mathrm{c} h$ a $\mathrm{f} f \mathrm{e} n \mathrm{~h}$ e it kann man aus unseren Beobachtungen Folgendes erschliessen. Auf dem losen, ungemischten Schlammgrund fehlt zwar S. gaimardii stellenweise nicht, doch ist sie dort nicht häufig; bemerkenswert sind die 4 Funde (einmal, St. 130, in sehr grosser Individuenzahl) in Schlamm mit modernen Pflanzenteilen. Am häufigsten fanden wir das Tier auf mit Steinen oder Kies gemischtem, meist härterem Schlammgrund. $\mathrm{Ob}$ dies darauf zurückzuführen ist, dass die Art solchen Boden bevorzugt, oder nur darauf, dass reiner Steinboden in den etwas grösseren Tiefen des Eisfjords selten ist, mag dahingestellt sein. An den steinigen, mit Laminarien bewachsenen Stellen in der oberen Uferzone fanden wir nur einmal (St. 5) zwei Lxemplare; da zahlreiche solche Stationen untersucht wurden, muss die Art dort äusserst selten sein. Dagegen fanden wir sie oft an steinigem Grund mit Balanus porcatus-Kolonien (4 Stationen von 7) oder Lithothamnion (5 Stationen von 9); so häufig wie S. polaris $\mathrm{u}$. a. ist sie jedoch dort kaum.

Die Verteilung der Fundorte in vertika le r Richtung wird durch die nachstehende Ubersicht veranschaulicht. Sie zeigt, dass $S$. gaimardii in 'Tiefen von 2 bis etwa 
$260 \mathrm{~m}$ angetroffen wurde. In der Uferzone lebt sie jedoch nur ganz sporadisch; während der zahlreichen Dredgungen oberhalb von $20 \mathrm{~m}$ wurde sie nur viermal, jedesmal in 1 oder 2 Exemplaren, gefunden. Zwischen 20 und $30 \mathrm{~m}$ wird sie plötzlich häufig und gehört von nun an wenigstens bis in $150 \mathrm{~m}$ Tiefe zu den häufigsten Arten. Die kleinen Unregelmässigheiten, die im Schema in die Augen springen, haben natürlich keine Bedeutung; die auffallend zahlreichen Funde zwischen 30 und $40 \mathrm{~m}$ beweisen z. B. nicht,

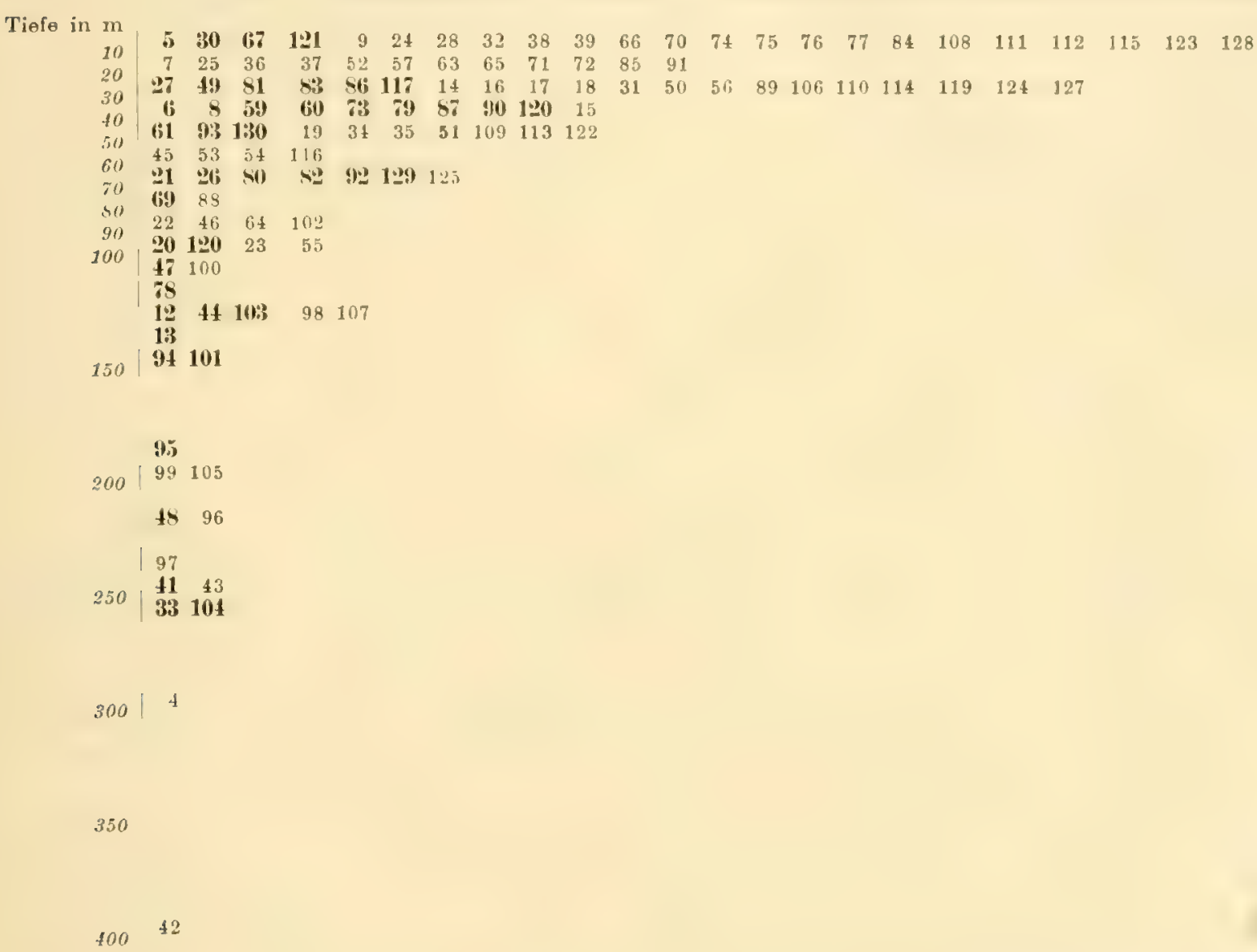

dass die Art hier häufiger als zwischen 40 und $60 \mathrm{~m}$ lebt, denn zwischen 60 und $70 \mathrm{~m}$ findet man ja wiederum viele Fundorte. In der unterhalb von $150 \mathrm{~m}$ liegenden Schicht (bis $260 \mathrm{~m}$ ) ist die Art nicht selten. Etwas Bestimmteres kann man wegen der verhältnismässig wenigen in dieser Tiefe vorgenommenen Dredgungen nicht sagen, doch scheint sie hier nicht ganz so häufig wie in geringerer Tiefe zu sein. Dieses mutmassliche Seltenerwerden nach unten zu beruht vielleicht nur darauf, dass hier der lose Schlammboden ïberwiegt.

Einige Unterschiede zwischen den aus verschiedenen Tiefen stammenden Exemplaren habe ich nicht bemerkt. Die Grösse ist wenigstens von 40 bis $200 \mathrm{~m}$ oder mehr dieselbe (bis mehr als $60 \mathrm{~mm}$ ). Oberhalb von $30 \mathrm{~m}$ (mit einer Ausnahme sogar oberhalb von $40 \mathrm{~m}$ ) fanden wir jedoch keine Exemplare von mehr als $47 \mathrm{~mm}$ Länge und keine eier-

K. Sv, Tet. Akad. Handl. Tand 54, N:a 7. 
tragenden Weibehen. Dies beweist natiurlich nicht, dass die hier herrsehenden Bedingungen eine geringere Grösse hervorrufen (wie bei S. polaris), wohl aber, dass fortpflanzungstätige 'Tiere sich wenigstens im Sommer nicht dort aufhalten.

Die Abhängigkeit von den im Eisfjord herrschenden Temperaturverhältnissen geht aus der folgenden Übersicht hervor. S. gaimardii wurde demnach in Wasser von allen beobachteten 'Iemperaturen angetroffen. In mehr als + $3^{\circ}$ warmem Wasser ist sie jedoch sehr selten; zwischen + 3 und etwa - 0,9 ist sie iiberall mehr oder weniger häufig, während sie in noch kälterem Wasser nur zweimal gefunden wurde. Der letztere Umstand bedentet zweifellos nicht, dass die Art die niedrigsten 'Temperaturen meidet; dass dies nicht der Tall ist, kann man teils aus der grossen Anzahl und der Grösse der auf St. 101 und 120 gefangenen Exemplare, teils aus der allgemeinen Verbreitung schliessen. Das Fehlen an 6 der 8 kältesten Stationen dürte leicht durch die unguinstige Bodenbeschaffenheit und ähnliche Umstände zu erklären sein (St. 54, 55, 125: loser, roter Schlamm; St. 88, 100: nahe am Gletscherrand gelegen).

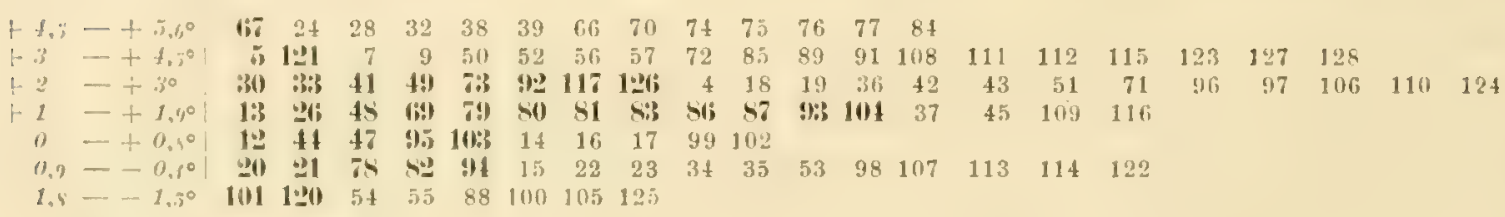

Schwieriger ist es, die Ursache der grossen Seltenheit in den oberen, im Sommer warmen Wassersehichten zu finden. Die Bodenbeschaffenheit ist meist nicht unginstig (zäher Schlamm oder Schlamm mit Steinen usw.; an steinigem Laminariengrund scheint die Art in andern Gegenden allgemein zu sein, vgl. Hansen 1887). Wenn die bathymetrische Verbreitung im Eisfjord durch die hohe Sommertemperatur des Oberflächenwassers eingeschränkt wird, so muss natürlich die obere Verbreitungsgrenze in Gegenden mit ähnlichen äusseren Bedingungen in derselben 'Tiefe, an hocharktischen (besonders nördlich gelegenen) Kiisten dagegen höher liegen. Hieriber kam gegenwärtig nichts Bestimmtes gesagt werden. In hocharktischen Gegenden, z. B. in Nordostgrönland (s. Stepilenswa 1913), ist die Art auffallend oft in 5-20 m Tiefe gefunden worden. Auch von Westgrönland liegen jedoch mehrere Angaben iiber solche Funde vor, und auch davon abgesehen, kann man nicht behaupten, dass die Art in kälteren Gegenden höher als im Lisfjord hinaufsteigt, denn auch hier findet sie sich ja spärlich noch bis zu $5 \mathrm{~m}$ hinauf.

Die Sachlage wird noch verwickelter durch die merkwïrdige 'Tatsache, dass S. gaimardii in der borealen Region eine reine Litoralform ist, die schon in einer Tiefe von wenigen Metern lebt (s. unten). Diese Veränderung in der Vertikalverbreitung wird von Apreltöf (1906, S. 201 ) als eine Anpassung an die borealen Temperaturverhältnisse erklärt. S. gaimardii ist nach ihm eine solche Kaltwasserform, die nur für ihre Entwicklung kaltes Wasser nötig hat. Diese findet warhrscheinlich im Winter statt, und die Art bewohnt daher an den borealen Küsten ausschliesslich die Uferzone, weil das Wasser dort während der kalten Jahreszeit die niedrigste 'l'emperatur hat, die das Meer in diesen Gegenden iiberhaupt je aufweist. Diese Hypothese erklärt ja sehr schön die sonst sehwer 
verständliche Vertikalverbreitung der borealen Tiere, dagegen nicht, warum die Art im Eisfjord eben in der Zone sehr selten ist, die im Süden ihre ausschliessliche Heimat bildet.

Wenn die Temperaturverhältnisse auch hier mit im Spiele sind, muss man sich die Sache folgendermassen vorstellen: die Embryonalentwicklung erfordert unwiederruflich sehr kaltes Wasser (eine Temperatur von 4 bis $+6^{\circ}$ scheint nicht zur genuigen); die erwachsenen Tiere bevorzugen Wasser von weniger als $+3^{\circ}$ Temperatur, sie haben sich aber, wenn solches Wasser nicht zu Gebote stand, an wesentlich höhere Wärmegrade gewöhnen können, was in bezug a uf die Entwicklung nicht möglich war. Solange die Lebensweise unter hocharktischen Bedingungen so unvollständig bekannt ist, lässt sich diese Annahme jedoch nicht beweisen.

Fortpflanzung, Entwicklung. Eiertragende Weibehen finden sich von folgenden 15 Stationen: 13, 26, 33, 21, 94, 92, 93, 79, 80, 101, 47, 44, 95, 69, 130. Das Fehlen an den übrigen Stationen beruht, wie ein genauerer Vergleich der Fundergebnisse lehrt, darauf, dass die an ihnen gefundenen Weibchen nicht oder eben erst die Grösse erreicht hatten, bei welcher die Geschlechtstätigkeit eintritt. Nur St. 48 bildet eine Ausnahme; dort wurden jedoch nur wenige (6) Exemplare, davon nur ein grösseres (steriles) Weibchen erbeutet. - Die kleinsten eiertragenden Weibchen messen $48 \mathrm{~mm}$.

Die Eier befinden sich durchwegs in unentwickeltem Zustande oder in den friihesten Entwicklungsstadien, sowohl an den Nitte Juli wie an den Ende August gefangenen Weibchen. Die Entwicklungszeit fällt also auch bei der arktischen Rasse von S. yaimardii nicht in den Sommer, sondern in die Zeit September bis . Funi. Im nördlichen Norwegen werden die Eier erst im Herbst abgesetzt (s. Dons 1915).

\section{Mllgemeine Verbreitung.}

(Fig. 4.)

$$
\text { S pitzbergengobiet: }
$$

Westspitzborgon: Hornsund, Bolsund, Eisford, (Krörer 1842, OnLix 1901, Brneta 1907, 1)ons 1915 und oben). Nordwostspitzborgon (HoEk 1882, SATs 1886, OnLm 1901). Ostspitzborgon: Storfjord und unmittelhar O. davon, Hopen Filand und SW. davon (DoFLerv 1900, OnLAN 1901, BrRUta 1907, DONS 1915).

Die Fundo der Helgoland-Expedition sind nach DOFLEIN alle nauf cinen kleinen Ram hei südost-śpitzbergen zusammengedrängt is (vgl. seine Kartenskizzo, S. 358). Wenigstens im Sommer 1898 wïrte die Art daher im ganzen Warmwassergebiet bei Westspitzbergen gefehlt haben. Von dieser Art gilt jedoch ganz dasselbe, das oben über S'. po'aris geäussert wurde; übrigens ist sie auch im Sommer $1898 \mathrm{im}$ Fisfjord gefunden worden (Nathorst. Expedition).

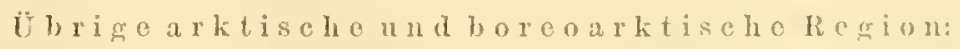

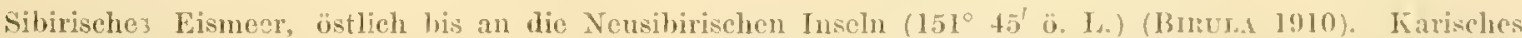
Heer (Stuxberg 1882, 1886, HANSEN 1887 a, Broul 1900, 1910). Karische Pforte, Matotschkin sehar, Wrst.

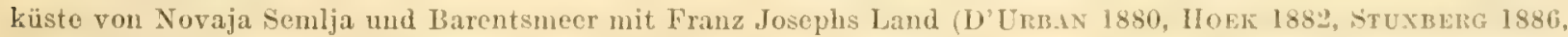
Scott 1899, Stembing 1900, Bmeula 1900, 1910, Stapers 1911, Doxs 1915). Meisses Mecr, Murnankïste (Jar-

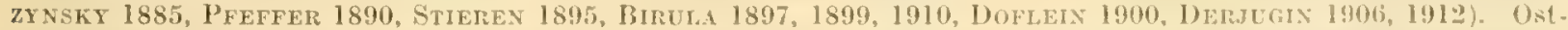

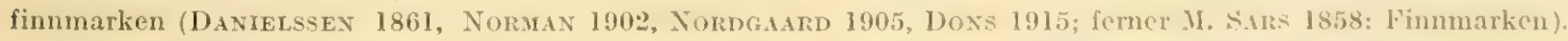
Kalte Fjorde in Wrestfinnmarken und dem Lofotengebiet (s. unten). - Ost- und Nordkifste von Island (IAxis

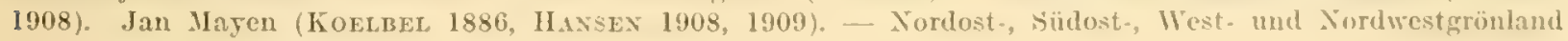


(STEPHEXSEN 1913, 1916 und frühere Autoren; hicher auch S. recurvirostris IIOLAXDER [1914], 8. STEPHENAEX 1916). - Arktisches Nordamerika: Ellesmere Land (Mrems 1877 a). Jones sund (SARs 1909). Barrow-Strasse (BEL, 1855). - Nordostküste von Labrador, Golf von St. Lawrence und Ostküste von Nordamerika bis K. Cod

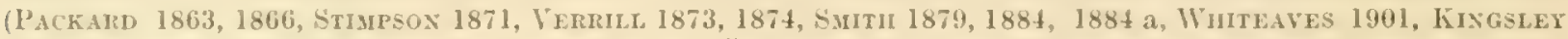
1901, IRAthibu 1904, 1905). - Pazifisches Gebiet: Óstlichster Teil der sibirischen Nordküste (STUXBarg 1882). Nordküsto von Maska, Beringsstrasse, Beringsmeer, Aleuten (RatnBUx 1904, ferner Stumsox 1860, MURDoci 1885).

$$
\text { Boreale liegion: }
$$

N orwogiseho W est $\mathrm{k}$ ï $\mathrm{s}$ e: Westfinnmarken und Jofoten, teils unter horcoarktischen, teils unter borealen Bedingungen (Lilljeborg 1851, G O. Sars 1886, Sparke-Schneider 1884, Aurivillius 1886, Nord. GAARD 1905, Frexr 1906, 1906 a, Doxs 1915). Nordland, 65 $40^{\prime}$ n. Br. (Doss 1915). Trondhjemsfjord (Srory 1878, 1880). Kristiansund, Molde (Krörer 1842, DanielsseN 1861). Fordsecküste (G. O. SARS 1872, METzGer

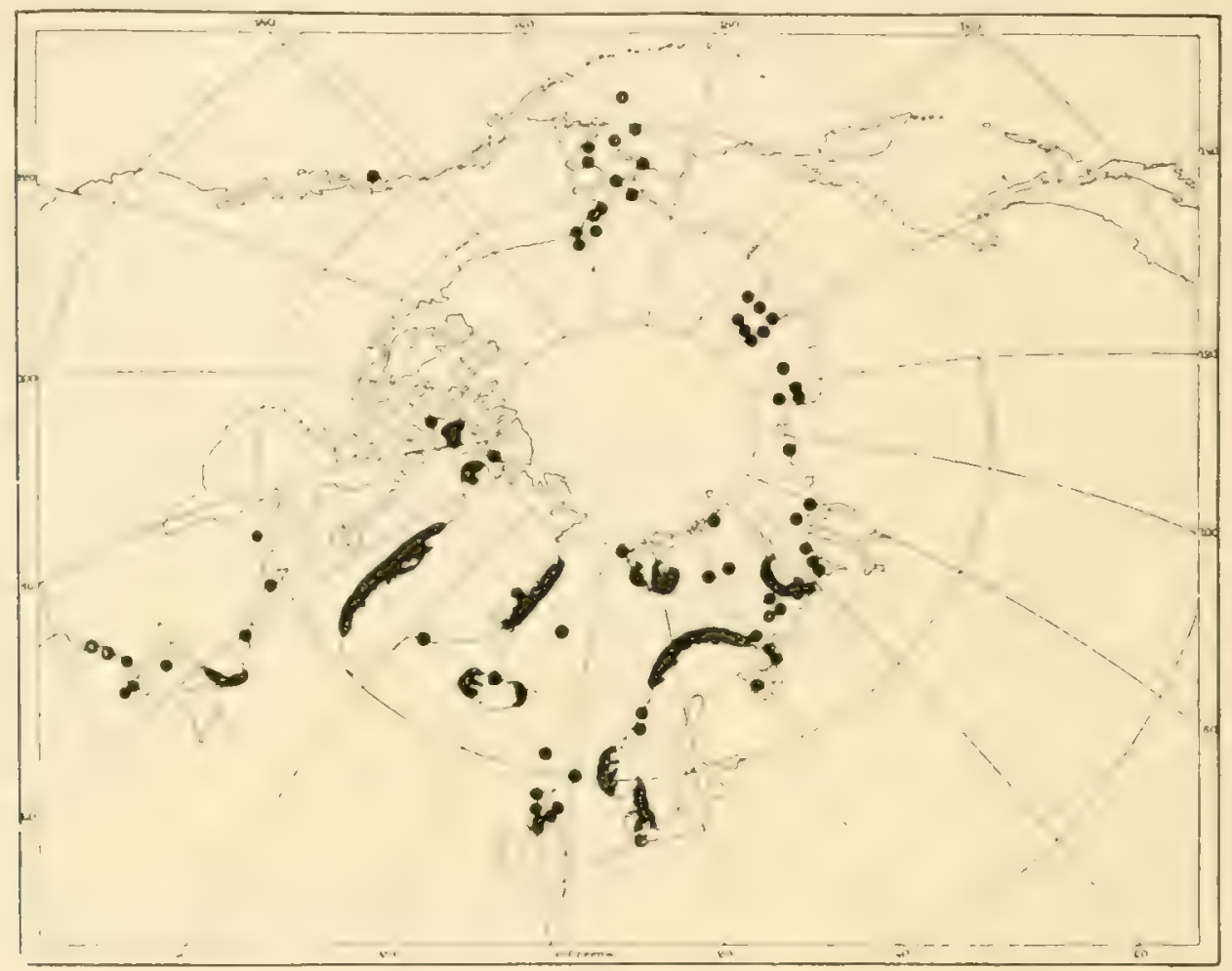

Fig. 4. Spirontocaris gaimardii.

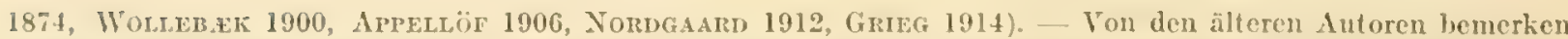
AI. SARS und DANierssex, dass $S$. gaimerdii an der ganzen Küuto allgemein ist; nach Krörkr ist sio hüufiger als irgend eine andere Spirontocaris-Art. Appeluör findet indessen, dass dieso Art ventschieden viel seltener ist als $S$. polaris.

Śk a gerak: Arendal (OrTmany 1890). Schwedische Küste (Kosterrine, Väderöarna, Gullmarfjord und Umgebung, Vinga) (Gö́s 1863, 'Try zом 1903, 'l'Héel 1907, LAGErberg 1908). K a t to g a t, sehr allgomein,

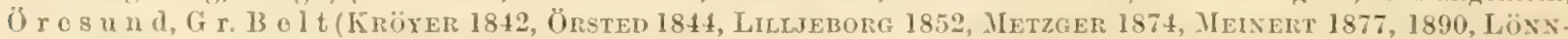
Berg 1898, 1903, Petersex \& Levinsex 1900, Stepinessex 1910, 1910 a, Björch 1913, 1913 a, 1915). Südwestlicho 0 s $\mathrm{s}$ ec: Kiclerbucht (IÖBIUS 1873, MEINERT 1890).

B ritiscli In seln: Ostküsto von Schottland (Firth of Forth, Aberdeen) (Scott 1888). Shetlandinseln, Hebriden (NoRMAN 1869, 1867). Westküste von Schottland (Firth of CIyde) (HENDERSON 1887, IIOTLE. 1890, Scott 1897,1897 a).

F ärö or (HANSEN 1908). Nordwest- und Westküste von I s l a nd (LUNDBeck 1893, HANSEN 1908).

Verbreitung in wa rmon 'T eil des pazifisehon Gebiotes: Küste ron Alaska southward to Sitkas; naho rerwadto Formen weiter südwärts, bis Washington (RaTnBU. 1904). 
Bathymetrische Verbreitung: In der arktischen Region umfasst die bathymetrische Verbreitung die Tiefen von $2 \mathrm{~m}$ (s. oben) bis etwa $300 \mathrm{~m}$ (175 Faden, s. HANsEx 1887, 1908; einige Angaben über Funde in noch grösserer Tiefe bedürfen, wie dieser Autor hervorhebt, noch der Bestätigung). In der Uferzone (bis etwa $20 \mathrm{~m}$ ) ist die Art in der Regel selten, möglicherweise jedoch nicht unter hocharktischen Bedingungen (s. oben). Zwischen 20 und 100 bis $150 \mathrm{~m}$ ist sie iiberall sehr häufig. In grösserer Tiefe, bis etwa 250 oder $260 \mathrm{~m}$, ist sie früher nicht oft gefunden worden; wahrscheinlich ist sie jedoch dort überall, wie im Eisfjord, zwar weniger häufig als in etwas geringerer Tiefe aber doch nicht selten.

In der bor e 1 e $n$ Region lebt $S$. gaimardii nach den übereinstimmenden Angaben der Autoren ausschliesslich in se hr geringer Tiefe, 4 oder $10-25 \mathrm{~m}$ (vgl. besonders Appeltöf 1906: „nicht tiefer als 25 m»; Meinert; Stepinensen 1910: "meist etwa $10 \mathrm{~m}$ "; LöNNBERG 1898, 1903: 13-25 m; LAGERBERG 1908: "einige wenige bis $40 \mathrm{~m}$ ). Nach einer Angabe von Trybom (1903) scheint die Art ganz ausnahmsweise bedeutend tiefer hinabzustejgen (Kosterrinne, 220 bis $230 \mathrm{~m}$ ).

\section{Pandalus borealis $\mathrm{Kr}$.}

\section{Verbreitung in Eisfjord.}

Verzeichnis der Fundorte (s. Karte 1):

\begin{tabular}{|c|c|c|c|c|c|c|c|}
\hline $\begin{array}{l}\text { Nr. } \\
\text { der } \\
\text { Stat. }\end{array}$ & Ort und Datum & Tiefe & $\begin{array}{l}\text { Wasser- } \\
\text { temperatur }\end{array}$ & $\begin{array}{c}\text { Salz- } \\
\text { gehalt } \\
0 / 00\end{array}$ & Bodenbeschaffenheit & Gerät & $\begin{array}{l}\text { Anzahl und } \\
\text { Grösse }\end{array}$ \\
\hline 42 & $\begin{array}{l}\text { Svensksundstiefe(Ein- } \\
\text { gang in den Fjord), } \\
\text { Nordseite . . . 24.7 }\end{array}$ & $406-395 \mathrm{~m}$ & $382 \mathrm{~m}:+2,61^{\circ}$ & 34,90 & Loser Schlamm & Trawl & $\begin{array}{l}96 \mathrm{Ex} ., 35-13 \mathrm{~mm} \\
73\end{array}$ \\
\hline 43 & $\begin{array}{l}\text { Svonsksundstiefe (Ein- } \\
\text { gang in den Fjord), } \\
\text { Südseite.....25.7 }\end{array}$ & $228-257 \mathrm{~m}$ & $228 \mathrm{~m}:+2,74^{\circ}$ & 34,90 & 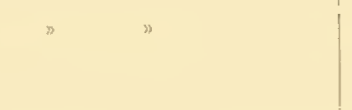 & Ottertrawl & $\begin{array}{l}\text { Etwa } 40 \\
\text { grosse } \mathrm{Fx} \cdot 1 \\
\text { bis } 145 \mathrm{~mm}\end{array}$ \\
\hline 4 & Srensksundstiefe $\quad .15 .7$ & $277-313 \mathrm{~m}$ & {$\left[\text { Etwa }+2,5^{\circ}\right]^{\mathbf{I}}$} & - & Schlamm & , & $\begin{array}{l}\text { Grosse Men- } \\
\text { gen ( } 7 \text { Liter, } \\
\text { wahrschein- } \\
\text { lich etwa } \\
1500 \text { Ex.) }\end{array}$ \\
\hline 13 & $\begin{array}{l}\text { Eingang in die Safo } \\
\text { Bay . . . 16.7 }\end{array}$ & $125-150 \mathrm{~m}$ & $144 \mathrm{~m}:+1,23^{\circ} \mathrm{II} \mid$ & 34,54 & \begin{tabular}{|} 
Schlamm mit Schalen; \\
Balanus porcatus.Ge- \\
meinsch.
\end{tabular} & Trawl & $\begin{array}{l}9 \mathrm{Ex}, 56- \\
73 \mathrm{~mm}\end{array}$ \\
\hline 33 & Fjordstamm . . . 23.7 & $263-256 \mathrm{~m}$ & {$\left[+2 \text { bis }+2,6^{\circ}\right]^{\mathrm{vur}}$} & - & Loser Schlamm & 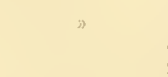 & $\begin{array}{l}103 \mathrm{Ex}, 35- \\
95 \mathrm{~mm}\end{array}$ \\
\hline 41 & $\triangle \quad . .24 .7$ & $234-254 \mathrm{~m}$ & $251 \mathrm{~m}:+2,56^{\circ}$ & 34,96 & * & , & $\begin{array}{l}67 \mathrm{Ex}, 30- \\
115 \mathrm{~mm}\end{array}$ \\
\hline 94 & $\begin{array}{l}\text { vor dem } \\
\text { Eingang in dio } \\
\text { Tundra Bay . . . } 21.8\end{array}$ & $147-141 \mathrm{~m}$ & $140 \mathrm{~m}:-0,62^{\circ}$ & $3 \frac{1}{4}, 49$ & $\begin{array}{l}\text { Loser Schlamm mit klei- } \\
\text { nen Steinen }\end{array}$ & - & $\begin{array}{l}5 \text { Ex., } 45- \\
70 \mathrm{~mm}\end{array}$ \\
\hline 98 & Nordarm $\cdot \cdot 27.8$ & $130-116 \mathrm{~m}$ & $115 \mathrm{~m}:-0,82^{\circ}$ & 34,40 & Loser Schlan.m & . & $\begin{array}{l}6 \mathrm{Lx} \cdot \text {, etwa } \\
50 \mathrm{~mm}\end{array}$ \\
\hline 99 & n & $197-190 \mathrm{~m}$ & $190 \mathrm{m:}+0,80^{\circ}$ & 34,72 & " & 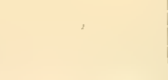 & $\begin{array}{l}10 \mathrm{Ex}, 36- \\
65 \mathrm{~mm}\end{array}$ \\
\hline 90 & $\begin{array}{l}\text { Nordarm, Eingang in } \\
\text { die Yoldia Buy } \quad 19.8\end{array}$ & $17-60 \mathrm{~m}$ & - & - & $\begin{array}{l}\text { Zäher Schlamm mit líos' } \\
\text { und Sand }\end{array}$ & Kl. Dredgo & 1. Ex., $35 \mathrm{~mm}$ \\
\hline
\end{tabular}




\begin{tabular}{|c|c|c|c|c|c|c|c|c|}
\hline $\begin{array}{l}\text { Nr. } \\
\text { dor } \\
\text { Stat. }\end{array}$ & Ort und & Datum & Tiefo & $\begin{array}{l}\text { Wasser- } \\
\text { temperatur }\end{array}$ & $\begin{array}{c}\text { Sulz- } \\
\text { gehnalt } \\
0_{\text {ofun }}\end{array}$ & Budenbeschaffenheit & Gesät & $\begin{array}{l}\text { Anzalıl unà } \\
\text { Grösse }\end{array}$ \\
\hline 93 & Ekman Ibay & $\cdots 20.8$ & $44-55 \mathrm{~m}$ & $+1,72^{\circ}$ & - & $\begin{array}{l}\text { Zälter, roter Schlamm. } \\
\text { Ftwas Stein }\end{array}$ & 'Trawl & ${ }_{5.5 \mathrm{~min}}^{6 \mathrm{~min}}$ \\
\hline 101 & Billen Bay & $\cdots 14.8$ & $150-140 \mathrm{~m}$ & $140 \mathrm{~m}:-1,67^{\circ}$ & $|34,43|$ & $\begin{array}{c}\text { Loser Schlan m mit Stei- } \\
\text { nen }\end{array}$ & $y$ & $\underset{\min }{2 \mathrm{Fx}_{\text {m }}, 58,72}$ \\
\hline 48 & Ostarm . . & . . 31.7 & $199-2.26 \mathrm{~m}$ & $210 \mathrm{~m}:+1,27^{\circ}$ & 34,72 & Loser Schlamm & - & $\begin{array}{l}150 \mathrm{Ex}, 3 \text { ? } \\
100 \mathrm{~mm}\end{array}$ \\
\hline 101 & Fjordstamm & $\ldots 17.8$ & $260 \mathrm{~m}$ & $\left|270 \mathrm{~m}:+1,62^{\circ}\right|$ & 34,79 & b & * & $\begin{array}{l}25 \mathrm{Lx}, 30- \\
35 \mathrm{~mm}\end{array}$ \\
\hline 44 & $\begin{array}{l}\text { Eingang in } \\
\text { vent Bay }\end{array}$ & dio Ad- & $150-110 \mathrm{~m}$ & $128 \mathrm{~m}:+0,01^{\circ}$ & 34,54 & Loser Schlamm mit Kies & * & $\mid \begin{array}{l}4 \mathrm{Ex}, 55- \\
75 \mathrm{~mm}\end{array}$ \\
\hline 95 & Fjordstamm & . . . 21.8 & $188-181 \mathrm{~m}$ & $\mid \begin{array}{l}{\left[\begin{array}{l}0 \\
\text { bis }\end{array}+0,5^{\circ}\right]^{\mathrm{vul}}} \\
\left(163 \mathrm{m:}-0,11^{\circ}\right)\end{array}$ & - & $\begin{array}{l}\text { Loser Sihlamm mit Stei- } \\
\text { nea }\end{array}$ & ○ & $\begin{array}{l}112 \mathrm{Ex}, 38- \\
90 \mathrm{~mm}\end{array}$ \\
\hline 97 & s & . . 23.8 & $243-230 \mathrm{~m}$ & {$\left[+2\right.$ bis $\left.+2,5^{\circ}\right] \mathrm{Vu}$} & - & Loser Schlamm & $\begin{array}{c}\text { Trawl } \\
\text { (Setz unllar) }\end{array}$ & $1 \mathrm{Ex}, 75 \mathrm{~mm}$ \\
\hline 96 & " & . . 22.8 & $\begin{array}{l}230 \text {-otwa } \\
200 \mathrm{~m}\end{array}$ & $208 \mathrm{~m}:+2,56^{\circ}$ & 34,76 & $\begin{array}{l}\text { Schlumm mit etwas Stoin } \\
\text { und Kies }\end{array}$ & Trawl & $\mid \begin{array}{c}3 \mathrm{Fx}, 45- \\
165 \mathrm{~mm}\end{array}$ \\
\hline 130 & Groon Iay & . 30.8 & $40-45 \mathrm{~m}$ & - & - & $\begin{array}{l}\text { Schlamm mit Algen- } \\
\text { rcisten }\end{array}$ & Iil. Dredgo & $\begin{array}{l}5 \mathrm{Ex}, 43 \\
47 \mathrm{~mm}\end{array}$ \\
\hline
\end{tabular}

Helgoland-Exped. 1898: Fjordstamm, vor dem Eingang in dio Coles Bay, 210 - 240 m, Schlamm; Srensksundstiefe, $365 \mathrm{~m}$, Schlamm (Dorlein 1900). Schwed. Exped.: 1898: Nordarm (südlich von unscrer st. 92), 175 $\mathrm{m}$, Schlamm; Srensksundstiefe, $400 \mathrm{~m}$, Temp. $+3^{\circ} ; 1900$ : Srensksundstiefe, $350 \mathrm{~m}$, Schlamm (OnLIX 1901). Russ. Exped. 1900: Fjordstamm vor der Advent Bay, $243 \mathrm{~m}$, 'Temp. (14.7) - 0,8 ; vor der Green Bar, $205 \mathrm{~m}$, 'Temp. (27.6) - 0,8 (Birula 1907). Ferner Dons 1915: "200-350 m» (2 Ex.).

Pandalus borealis wurde, wie die obenstehende Übersicht zeigt, im Eisfjord an 19 Stationen erbeutet. Er ist also dort häufig, im Grunde genommen - wenn man die beschränktere bathymetrische Verbreitung mit in Rechnung bringt — wohl ungefähr ebenso häufig wie Spirontocaris polaris und gaimardii.

Wir fanden diese Art ausschliesslich auf is e h 1 a $m$ m bo de $n$. Dies beweist jedoch nicht, dass sie nur auf solchem zu leben vermag, denn harter Boden fehlt nach unseren Beobachtungen so gut wie ganz in den von ihr bewohnten Tiefen. Dass sie in anderen Gegenden nicht ganz auf den Schlammgrund beschränkt ist, zeigen einige von HANSEN (1887) mitgeteilten Funde an der grönländischen Westkỉste. In den skandinavischen Fjorden lebt sie nach den Angaben von WOLLEBæe (1908) und BJörcK (1911) nicht ausschliesslich auf dem losen Schlammboden, wo sie gefischt wird, sondern auch auf steinigem und felsigem Boden sowie unter Korallen. Die Auffassung WoLLEBEK's scheint gut begrüdet zu sein und ist wahrscheinlich für alle Gegenden gültig: $P$. borealis lebt nicht ausschliesslich, aber vorwiegend auf Schlammboden; besonders während der Fortpflanzungszeit mag or jedoch sogar in grosser Menge auf steinigem Grund auftreten. - Im Eisford scheint die Art sogar den ungemischten Schlammgrund vorzuziehen; wo der Schlamm mit Steinen, Kies oder Schalen gemischt war, fanden wir sie mit einer Ausnahme (St. 95) nur in wenigen Exemplaren.

Die bathymetrische Verbreit ung erstreckt sich im Eisford von etwa $40 \mathrm{~m}$ (St. 130; St. 90 gehört in der Tabelle der Reihe $30-40 \mathrm{~m}$ an, die Dredge bewegte sich aber hier zwischen 17 und $60 \mathrm{~m}$ ) bis in die grösste Tiefe des Fjords $(400 \mathrm{~m})$. Die 
nachstehende Übersicht zeigt jedoch deutlich, dass die Art oberhalb von etwa $125 \mathrm{~m}$ äusserst selten ist; unter den zahlreichen dort vorgenommenen Dredgungen wurde sie nur dreimal, in zusammen 12 kleinen Exemplaren erbeutet. In den tieferen Teilen des Fjords muss sie ganz ausserordentlich häufig seir, da wir sie an fast allen dort untersuchten Stellen erbeuteten. Wenigstens unterhalb von etwa $180 \mathrm{~m}$ ist sie offenbar uiber

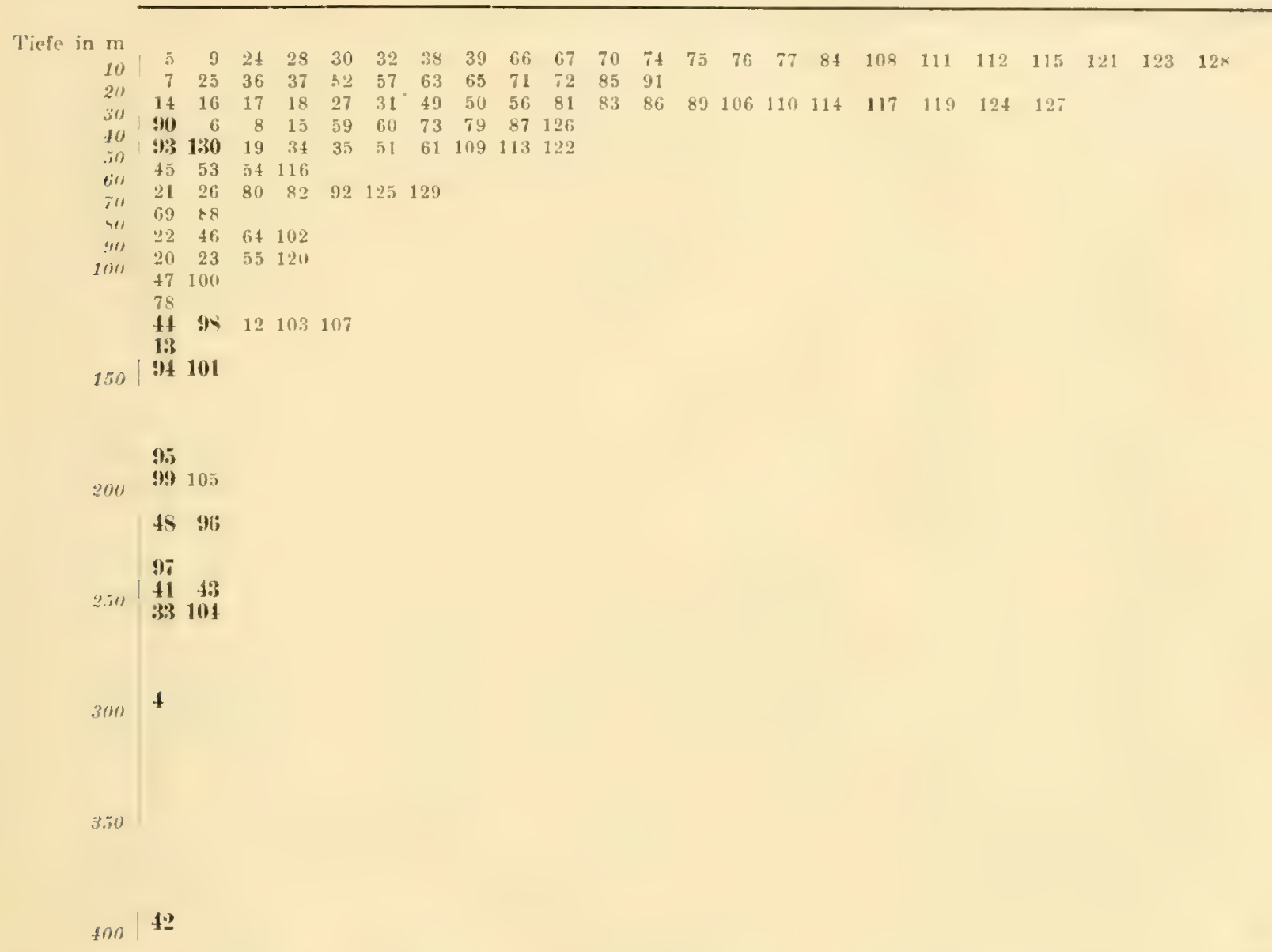

alle Tiefen gleichmässig verbreitet und meist in grosser Individuenzahl vorhanden; unterhalb von $200 \mathrm{~m}$ fingen wir sie sogar an allen Stationen, in zusammen wohl etwa 2000 Exemplaren. Zwischen etwa 125 und 150 bis $180 \mathrm{~m}$ ist sie auch nicht selten (an 5 von 8 Stationen), hier fanden wir sie jedoch nur in geringer Individuenzahl.

Pandalus borealis fehlt vollkommen in den im Sommer wärmsten Wasserschichten des Fjords; die höchste von uns gemessene Temperatur ist $+2,7 t^{\circ}$. Eine Einwirkung der Temperatur ist jedoch hier ausgeschlossen; die Art erträgt in andern Gegenden gut eine noch höhere Temperatur als die höchste Sommertemperatur des Eisfjords und fehlt iberall in seichtem Wasser, unabhängig von den Temperaturverhältnissen. Diese Art ist in der Tat ein vorzuigliches Beispiel dafuir, wie vorsichtig man die Methode der statistischen Analyse der Fundhedingungen handhaben muss, mn nicht den bedenklichsten Fehlschlüssen zum Opfer zu fallen. 
In Wasser von negativer Temperatur fanden wir P. borealis drei- oder (inkl. St. 44) viermal; an den übrigen 21 Stellen mit negativer Wassertemperatur fehlte sie ganz. Der erste Eindruck, dass sie das kälteste Wasser meide, verschwindet jedoch zum grossen 'I'eil, wenn man diese 21 Stationen näher betrachtet; die meisten liegen entweder in einer Tiefe (oberhalb von $75 \mathrm{~m}$ ), wo das 'Tier in der ganzen arktischen Region sehr selten ist (11 Stationen) oder wurden nur mit einer kleinen Dredge untersucht (7 Stationen). Trotzdem ist der erste Eindruck, dass die Art vorwiegend dem wärmeren 'Tiefenwasser des Fjords angehört, zweifellos zrichtig. Der Grund für diese Annahme ist dreifach: Erstens fanden wir an den Stellen mit negativer Wassertemperatur nur vereinzelte Exemplare, ein reichliches Material und grosse Exemplare ausschliesslich in dem wärmeren, atlantischen Wasser. ZWweitens fällt die obere Grenze für das regelmässige Vorkommen mit der unteren Grenze der intermediären Kaltwasserschicht zusammen; in andern arktischen Gegenden scheint das 'Tier etwas höher hinaufzusteigen (s. unten). Drittens lehrt eine Analyse der allgemeinen V'erbreitung, dass die Art hocharktische Bedingungen meidet (s. unten).

Das Vorkommen im kältesten Wasser des Fjords (besonders an St. 101, - 1,67 ${ }^{\circ}$ ) muss folglich dahin gedeutet werden, dass die Art spärlich und kiimmerlich - oder zeitweise, vielleicht ausserhalb der Fortpflanzungszeit - hocharktische Bedingungen auszuhalten vermag; die Stellen mit warmem und kaltem Wasser befinden sich ja im Eisfjord in geringer Entfernung voneinander. Nach unseren Beobachtungen zu urteilen, bevorzugt die Art vielleicht sogar Wasser von mehr als etwa $+1^{\circ}$ Temperatur; einmal fanden wir sie zwar häufig in wahrscheinlich kälterem Wasser (St. 95), diese Stelle lag aber ganz an der Grenze zum warmen 'Tiefenwasser.

Fortpflanzung, Entwicklung. Eiertragende Weibchen fehlen in unserem Material fast vollkommen; nur von St. 41 (24.7) besitzen wir ein Weibchen mit ganz unentwickelten, also wahrscheinlich vor kurzem abgesetzten Eiern. Unser Material an geschlechtsreifen Tieren ist indessen sehr umbedeutend (die bei St. 4 und 43 gefangenen wurden nicht aufbewahrt und ich versäumte leider, einige Notizen über den geschlechtlichen Zustand der Weibchen zu machen). In dem reichlichen, von der Kolthoff-Expedition Ende Juni 1900 im Eisfjord gesammelten Naterial finde ich kein einziges eiertragendes Weibchen. Die Eier werden also jedenfalls nicht vor Juli abgesetzt. Dagegen ist es unmöglich zu entscheiden, ob das von uns am 24.7.1908 gefundene eiertragende Weibchen seine Eier abnorm früh abgesetzt hatte oder ob die Fortpflanzungszeit im Eisfjord friher eintritt als in der borealen Region, wo man nach WOLLEB æK und BJörck erst Anfang September eiertragenden Weibchen begegnet (der letztere Autor fand eimmal schon am 16.8 zwei solche Exemplare). A priori ist es nicht unwahrscheinlich, dass die Fortpflanzungszeit ein wenig früher als im borealen Gebiet der skandinavischen Küste beginnt; dafür sprechen einzelne Beobachtungen in andern arktischen Gegenden (eiertragende Weibchen in Nordwestspitzbergen am 19.8., im Beringsmeer am 5. und 6.8 beobachtet, s. Birula 1907, Patirbun 1904) und vor allem in Nordnorwegen, wo eiertragende Weibchen vom August an angetroffen werden (s. KIÆR 1906 a, Dons 1915 u. a.). Die Untersuchungen BJörck's (1911) haben gezeigt, dass schon innerhalb eines kleinen Gebietes Schwankungen in der Fortpflanzungszeit vorkommen können. 
Wenn die Entwicklung der Eier also wahrscheinlich in arktischen etwas früher als in borealen Gegenden beginnt, so muss sie nach dem oben Gesagten jedenfalls dort wie hier im Winter vorsichgehen.

\section{Allgemeine Verbreitung.}

(Fig. 5, 6.)

S pitzborgengebiet:

West spitzbergen: Wr. vom Hornsund, Eisfjord und W. davon, W. und N. vom Pr. Charles Foreland: Nordwestspitzbergen (SArs 1886, OnLix 1901, Breula 1907 und oben). Nordspitzborgen:

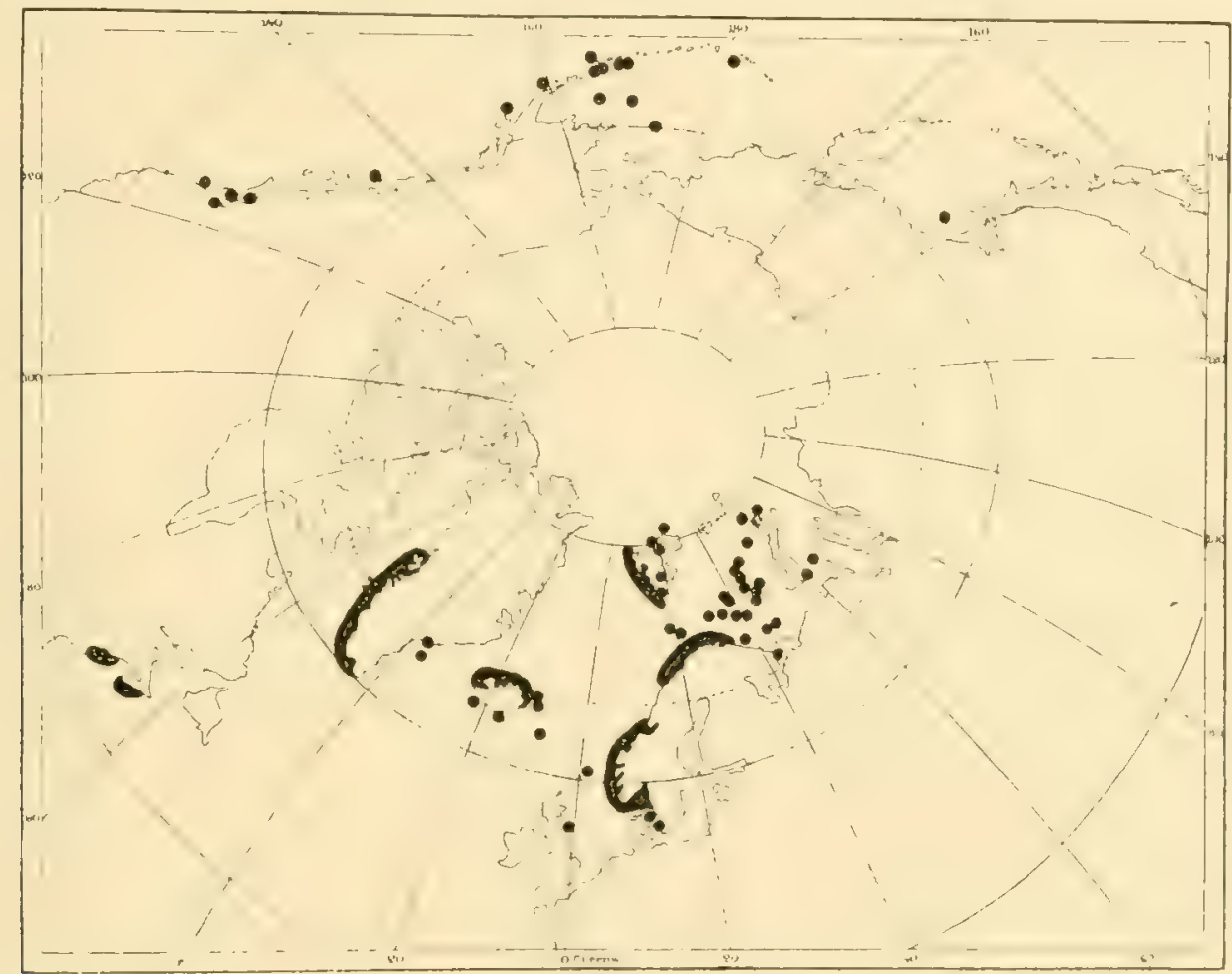

Fig. 5. Pandalus borealis.

Hinlopen-Strasse, nördlicher Teil (Dorteis 1900). N. vom Nordostland (Ornin 1901). Ostspitzbergen: Storfjord, 1 Fundort (Birula 1907). Zwischen dem Südkap und B e e re n L il a nd (SARs 1886).

Pandalus barealis bewohnt, wie Binula hervorhebt, vorzugswoise das warme Westgebiet. Ein näheres Stu. dium der bisherigen Beobachtungen lehrt, dass er nur sporadisch im Kaltwassergobiet auftritt und die rein hoch. arkischen Toile von Nord- und Ostspitzbergon vollständig meidet. Im Storfjord wurdo dio Art nur in einem einzigen Exemplar beobachtet; an dem F'undort bei den Sieben-Inseln nördlich vom Nordostland wurdo eine Temperatur von $+2^{\circ}$ gemessen. Auch in die Ifinlopen-Strasse dringt, wie Orlis bemerkt, wenigstens in gowissen Jahren atlantisches Wasser ein. - DofLErv zieht eigentümlicherweise aus den ihm vorlierenden Tatsachen einen ganz ent . megengesetzten Schluss, den ich nicht zu widerlegen brauche; $P$. borealis verhalte sich "fast grenau " wie dio Spirontocuris-Arten, welche auf das Kaltwassergebiet eingeschränkt seien.

1 OmLIx schreibt "King Charles Land", sowohl die Longitud- und Latitudangabe wie die Angaben über Fangzeit und Wassertemperatur zeigen jedoch. diss dies Schreibfehler für l'rinz Charles Foreland ist.

K. Sr. Pet. Akal. Mandl. Tand $54 . x+07$. 
i) hrige arktischound boreoarktische Region:

Karisches Meer (HAXsEx 1887 a; nur 2 Exemplare). Barentsmeer: nordöstlicher Teil (HELLER 1875 [nach der Fangzeit, keino Lokalangabo], Kxipowntscu 1901); mittlerer und sïdlicher 'Teil bis vor Ostfiunmarken und der

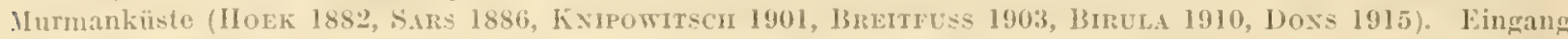
in das Weisse Meer (Dofleix 1900). Murmanküste (Jomyxxsky 1885, Brrula 1897, 1899, Dersugix 1906.

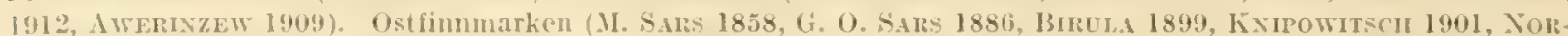

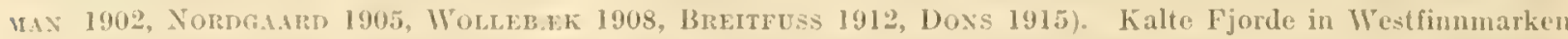

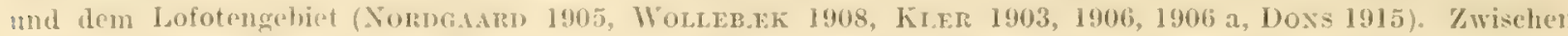
Becren Eiland und Jorwegen (S.me 1886, OHLIs 1901). - Fïrö-Island-Rücken, Ost-und Jordküste von Island

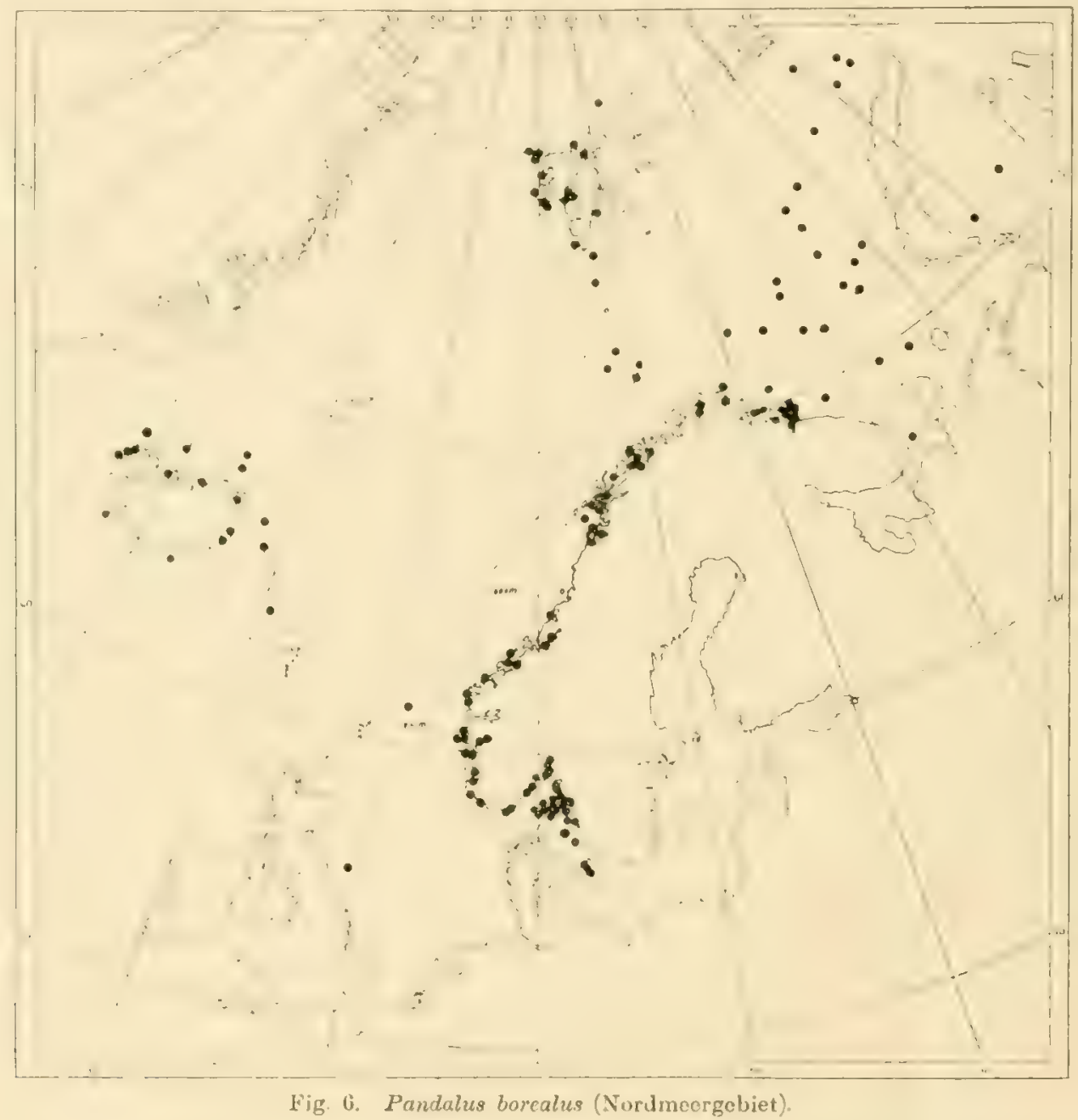

und Meer ron diesen Küsten (IANsex 1908, auch Scmudp 190.t). - Südostgrönland, Westgrönland nördlich

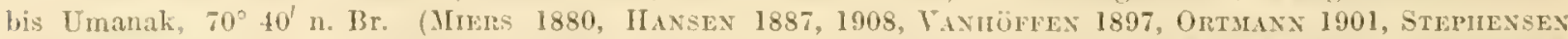
1912 a, 1913, 1916). 一 Ostkïste ron Nordamerika: Vor N. Scotia, Golf ron Maine bis K. Cod (Verrile 1873, 1874,

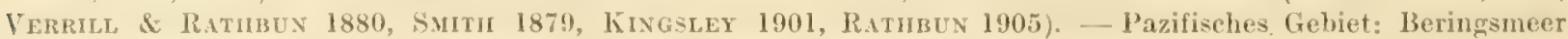
Alenten (Ratinur 1901). Ochotskischeg Ifeer (Brand 1851; keine Lokalangabe, dis Lage des Fundortes auf der Karto Fig. 5 daher unsicher.

Borealo Rogion:

Westküstevon Norwegen: Lofotengehiet (Nordainn 1905, Doxs 1915). Foldenfjord, Tromehjemsfjord, Kristiansund, Aalesund-Fjorde, Faafjord, Yordalsford, Sornefjorl, Fjordo in der Umgebung ron 
Bergen, Hardangerfjord, Stavanger-Fjorde, Stolsfjord, Rosfjord, Grönsford (G. O. SAls 1872, 1899, MIETzGEn 1874, StorM 1880, 1881, 1901, APPELLör 1906, NomdaAard 1912, (†RIEG 1914, vor allem IWorlebak 1908).

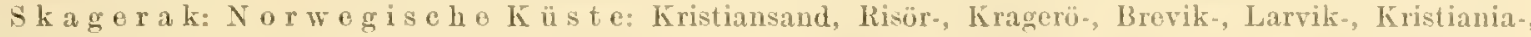
Drammenfjord (WoLLEB.E 1908; im Kristianiafjord schon von M. SARs [1866] und G. O. SATs [1869] häufig gefunden). S $\mathrm{ch}$ w e d is ehe $K$ ü s te: Säcken N. von Strömstad, Kosterrinne, Knapparna, Väderö-, Giullmar-, Kolje-, Borgila-, Kalföfjord (s. vor allem BJörcK 1911, 1913; vom Gullmarfjord und von Väderöarna ist $l^{\prime}$. borealis

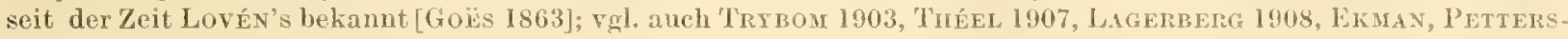

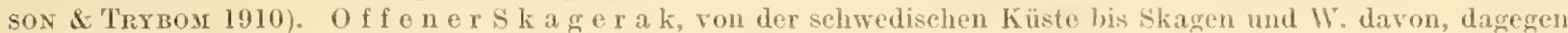
- wenigstens in der Regel, $\nabla g l$. den Allgemeinen Teil - nicht im atlantisehen Wasser dor grössten Skareraktiefe (Meinert 1890, Petersen \& Levinsen 1900, Tiry bou 1903, Stepilexsen 1910, 1910 a, Ekmax, Pettersson \& 'Trувом 1910, Влӧrск 1911, 1913).

K a t t e g a t: Nördlichster Teil, bis Tinga (TRY Bos 1903, Bзörck 1911, 1913). SO. von Särö und N. von Anholt ( $56^{\circ} 52^{\prime} \mathrm{n}$. Br.) (Petersen \& Levinsen 1900, Steprensen 1910, $1910 \mathrm{a}$, Bӟ̈rck 1911, 1913). Ö resu u d, drei Lokalitäten bis etwas S. von Hren (BјöreK 1915).

Nor d s e e, ein vereinzelter Fundort vor der Küsto fon Northumberland (103 m, 20 Lx.) (Norman 1909, NORMaN \& BRADY 1911).

Verbreitung im wamen Teil des pazifischen Gebiets: Aleuten bis Columbia River (RATIBUN 1901).

Bathymetrische Verbreitung: In der a r k ischen Region ist $P$. borealis in Tiefen von etwa $40 \mathrm{ml}$ (s. oben; vgl. auch HANSEN 1908) bis $900 \mathrm{~m}$ (HaNSEN 1908) gefangen worden. Er ist häufig bis in 'Tiefen von etwa $500 \mathrm{~m}$; aus grösserer Tiefe als $560 \mathrm{~m}$ liegt nur ein vereinzelter Fund vor. Im oberen Teil ihres Verbreitungsgebietes ist die Art wenigstens im Sommer iiberall selten; es scheint jedoch, dass die Grenze für das häufige Vorkommen bisweilen etwas höher als im Eisfjord (120 m) liegt, etwa bei 75 bis $90 \mathrm{~m}$ (s. z. B. Rathbun). In der b o re a 1 e $n$ R e g i o $n$ ist die untere Grenze ungefähr dieselbe wie in den arktischen Neeren. Nach WoLLEBæE (1908) (vgl. auch BJöRcK 1911 ) lebt die Art vorwiegend in Tiefen bis etwa $200 \mathrm{~m}$; wahrscheinlich gilt dies jedoch nur für gewisse Gegenden (Skagerak u. a.), wo die tieferen Wasserschichten eine unguinstige Beschaffenheit haben. In bezug auf die obere Grenze scheint wenigstens auf den ersten Blick ein Unterschied gegeniiber den arktischen Gegenden zu bestehen, indem die Art bereits in einer Tiefe von 15 bis $20 \mathrm{~m}$ angetroffen worden ist und schon bei $35 \mathrm{~m}$ in grosser Menge auftreten kann (WoLLEBFK, BJöRck). Dieser Untersehied ist jedoch vielleicht nur scheinbar. Sowohl die norwegischen wie die späteren schwedischen. Untersuchumgen zeigen, dass die Art in der Regel nur im Winter im seichteren Wasser der Fjorde angetroffen wird; sie wird gleichzeitig selten in den tiefsten 'Teilen und scheint also von den hydrographischen Verhältnissen bedingte vertikalo Wanderungen vorzunehmen (WOLLEBzK, BJöRCK).

\section{Sclerocrangon boreas (PHipes).}

Verbreitung im Eisfjord.

Verzeichnis der Fundorte (s. Karte 2):

\begin{tabular}{|c|c|c|c|c|c|c|c|}
\hline $\begin{array}{l}\text { Nr. } \\
\text { der } \\
\text { Stat. }\end{array}$ & Ort und Datum & Tiefo & $\begin{array}{l}\text { Wasser- } \\
\text { temperatur }\end{array}$ & $\begin{array}{l}\text { Sulz. } \\
\text { gehalt } \\
\text { \%/vo }\end{array}$ & Bodenbeschaffenhoit & Gerät & $\begin{array}{c}\text { Anzuhl und } \\
\text { Grösse }\end{array}$ \\
\hline
\end{tabular}

$43 \mid$ Stensksundetiefe(Fin- $|228-257 \mathrm{~m}| 228 \mathrm{~m}:+2,74^{\circ} \quad 34,90$ Loser Schlamm gang in den Fjord), Siidsoite...

Ottertraw! I Lx. (ziem. lich gross 


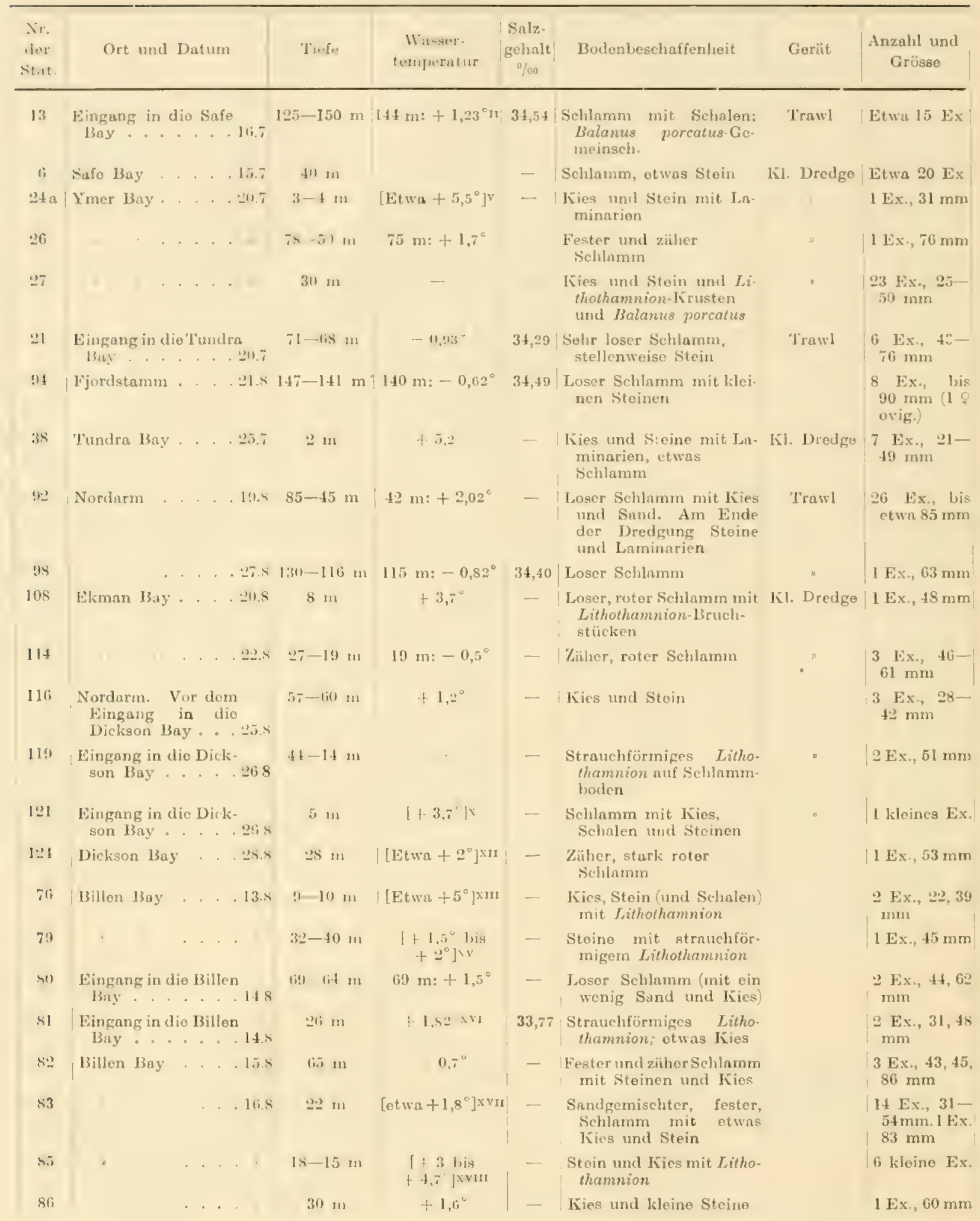




\begin{tabular}{|c|c|c|c|c|c|c|c|c|}
\hline $\begin{array}{l}\text { Nr. } \\
\text { der } \\
\text { Stat. }\end{array}$ & Ort und & Datum & Tiefe & $\begin{array}{l}\text { Wasser- } \\
\text { temperatur }\end{array}$ & $\begin{array}{c}\text { Salz: } \\
\text { gehalt } \\
\%\end{array}$ & Bodeubeschaffenheit & Gerät & $\begin{array}{l}\text { Anzahl und } \\
\text { Grösse }\end{array}$ \\
\hline 49 & Sassen Bay, & Bank . 31.7: & $\begin{array}{l}24-19 \mathrm{nnd} \\
19-28 \mathrm{~mm}\end{array}$ & {$\left[+2 \text { bis }+3^{\circ}\right]^{\mathrm{xtx}}$} & - & $\begin{array}{l}\text { Stein, Kies und Schalen } \\
\text { mit Lithothamnion }\end{array}$ & 'Trawl & $\begin{array}{l}\text { Etwa } 90 \mathrm{Ex}, \\
21-23 \mathrm{~mm}: \\
4 \text { Ex., y2- } \\
113 \mathrm{~mm}(3 \\
\text { \% ovig. }\end{array}$ \\
\hline 56 & $\begin{array}{l}\text { Tempel Bay. } \\
\text { Hafen : }\end{array}$ & Bjonas & Etwa $30 \mathrm{~m}$ & $35 \mathrm{~m}:+3,78^{\circ}$ & 34,33 & $\begin{array}{l}\text { Fester, braunroter } \\
\text { Schlamm mit Stoinon }\end{array}$ & KI. Dredge & I Ex., $43 \mathrm{~mm}$ \\
\hline 47 & $\begin{array}{l}\text { Eingang in di } \\
\text { Bay . . }\end{array}$ & ie Sassen & $97-120 \mathrm{~m}$ & 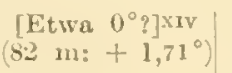 & 34,18 & Loser Schlamm & Ottertrawl & $\begin{array}{l}6 \mathrm{Ex}, 58- \\
64 \mathrm{~mm}\end{array}$ \\
\hline 44 & $\begin{array}{l}\text { Eingung in } \\
\text { vent Bay }\end{array}$ & dio Ad- & $150-110 \mathrm{~m}$ & $128 \mathrm{~m}:+0,01^{\circ}$ & 34,54 & Loser Schlarnm mit Käies & Trawl & $\begin{array}{l}\text { Etwa } 55 \mathrm{Ex}, \\
\text { bis } 88 \mathrm{~mm} \\
\text { (od. viel- } \\
\text { leicht molir: } \\
\text { nur einige } \\
\text { Ex. aufbe- } \\
\text { waint) }\end{array}$ \\
\hline 45 & Advent Bay & . . 28.7 & $70-42 \mathrm{~m}$ & $41 \mathrm{~m}:+1,85^{\circ}$ & 31,18 & $\begin{array}{l}\text { Loser, aber züher } \\
\text { Schlamm }\end{array}$ & $"$ & $\begin{array}{l}4 \text { Ex., } 45 \\
\text { Ex.) und } 70 \\
\text { mom }\end{array}$ \\
\hline 72 & $"$ & $\cdots .10 .8$ & $\begin{array}{c}11,15 \text { und } \\
19 \mathrm{~m}\end{array}$ & {$\left[+3 \text { bis }+4^{\mathrm{c}}\right]^{\mathrm{XxI}}$} & - & Sehr loser Schlamm & Kl. Dredgo & $\mid \begin{array}{l}3 \mathrm{Ex} \cdot, 27- \\
36 \mathrm{~mm}\end{array}$ \\
\hline 73 & w &. .11 .8 & $3 \tilde{-30} \mathrm{~m}$ & $\mid \begin{array}{l}\quad[+2 \text { bis } \\
\left.+2,7^{\circ}\right]^{\mathrm{XXII}}\end{array}$ & - & $\begin{array}{l}\text { Balanus porcatus-Ge- } \\
\text { meinsch.; líiesund Stein' }\end{array}$ & N & $\begin{array}{l}3 \text { Ex., } 75- \\
82 \mathrm{mmi} 10 \\
\text { kleine Ex. }\end{array}$ \\
\hline 95 & Fjordstamm & $\cdots 21.8$ & $188-181 \mathrm{~m}$ & $\begin{array}{l}{\left[0 \mathrm{bis}+0,5^{\circ}\right] \mathrm{vrr}} \\
\left(163 \mathrm{~m}:-0,11^{\circ}\right)\end{array}$ & 31,47 & Schlamm mit Steinen & 'Trawl & $\begin{array}{l}19 \mathrm{Ex}, \text { bis } \\
80 \mathrm{~mm}\end{array}$ \\
\hline 69 & Coles Bay. &. .8 .8 & $71 \mathrm{~m}$ & $\left|\begin{array}{c}{[+1,5 \text { bis }} \\
\left.+2^{\circ}\right] \times \times \mathrm{xIV}\end{array}\right|$ & - & $\begin{array}{l}\text { Kies, Stein und Schalen. } \\
\text { Etwas Lithothamnion }\end{array}$ & Iil. Dredge & $\begin{array}{l}5 \text { Ex., } 40- \\
63 \mathrm{~mm}\end{array}$ \\
\hline 71 & » & $\cdots,>1$ & $\begin{array}{c}14-16 \mathrm{~m} \\
\text { and } 16-14 \mathrm{~m}\end{array}$ & $\begin{array}{l}{[+2,4 \text { bis }} \\
\left.+3,5^{\circ}\right]^{\mathrm{NSV}}\end{array}$ & -- & $\begin{array}{l}\text { Zuerst Kies, dann } \\
\text { Schlamm und Iries }\end{array}$ & $"$ & $\mid \begin{array}{l}38 \text { Ex., } 19- \\
53 \mathrm{~mm}\end{array}$ \\
\hline 126 & Fjordstamm & $\ldots 30.8^{\prime}$ & $47-31 \mathrm{~m}$ & {$\left[+2\right.$ bis $\left.+3^{\circ}\right] \times x v y$} & - & $\begin{array}{l}\text { Balanus porcalus-Ge- } \\
\text { meinsch. }\end{array}$ & " & ${ }_{54 \mathrm{~mm}}^{6}$ Ex, $29-$ \\
\hline 127 & $"$ & ..." & $25 \mathrm{~m}$ & $\begin{array}{l}{[+3 \text { bis }} \\
\left.+3,5^{\circ}\right] \times \mathbf{X Y I}\end{array}$ & - & Zäher Schlamm & ” & $\begin{array}{l}11 \mathrm{Ex}, 2 \mathrm{I}- \\
38 \mathrm{~mm}\end{array}$ \\
\hline 129 & 2 & ... " " & $65 \mathrm{~m}$ & - & - & $\begin{array}{l}\text { Sandgemischter Schlamm } \\
\text { mit Kies und modern- } \\
\text { den Algenresten }\end{array}$ & , & $\begin{array}{l}5 \text { Ex., 37- } \\
47 \mathrm{~mm}\end{array}$ \\
\hline 103 & $\begin{array}{l}\text { Green Bay, } n \\
\text { Eingang . }\end{array}$ & ahe boim & $130 \mathrm{~m}$ & $+0,55^{\circ}$ & - & $\begin{array}{l}\text { Loser Schlamm. Einige } \\
\text { Steine und Balanus } \\
\text { porcatus }\end{array}$ & 'Trawl & $\begin{array}{l}28 \mathrm{Ex}, 40- \\
93 \mathrm{~mm}(1 \% \\
\text { ovig })\end{array}$ \\
\hline 130 & Green Bay. & $\ldots 30.8$ & $40-45 \mathrm{~m}$ & - & - & $\begin{array}{l}\text { Schlamm mit Algen- } \\
\text { rester }\end{array}$ & IKl. Dredge & $\begin{array}{l}9 \text { Ex., 36- } \\
65 \mathrm{~mm}\end{array}$ \\
\hline 60 & $"$ & $\ldots 3.8$ & $33 \mathrm{~m}$ & - & - & $\begin{array}{l}\text { Kies, Stein und Schalen } \\
\text { mit Lithothamnion- } \\
\text { IKrusten; zahlreiche Ba- } \\
\text { lanus porcatus-Irolonien }\end{array}$ & $"$ & $\begin{array}{l}\text { Etwa } 60 \text { Ex. } \\
\text { einige selir } \\
\text { gross } \\
\text { ovig.? die } \\
\text { ubrigen } \\
\text { klein }\end{array}$ \\
\hline 61 & $b$ & $\ldots .8$ & $46-35 \mathrm{~m}$ & - & - & $\begin{array}{c}\text { Kies und Stein. Balanus } \\
\text { porcatus-Gemeinsch. }\end{array}$ & $"$ & $\begin{array}{l}65 \mathrm{Ex} \text {, bis } \\
82 \mathrm{~mm}\end{array}$ \\
\hline 65 & $"$ & . . . 5.8 & $15 \mathrm{~m}$ & $1 \quad-\quad 1$ & - & Loser Schlamm & 3 & $3 \mathrm{Ex}$ \\
\hline 67 & $"$ & .. 6.8 & $2 \mathrm{~m}$ & $\mid\left[\right.$ etwa $\left.+5^{\circ}\right] \times x v 11 \mid$ & - & $\begin{array}{l}\text { Loser Schlamm mit mo- } \\
\text { dernden Pflanzenteilen }\end{array}$ & s & $\begin{array}{l}14 \mathrm{Ex}, 14- \\
41 \mathrm{~mm}\end{array}$ \\
\hline
\end{tabular}

[Ausser im Eisfjord fanden wir einigo Excmplare im 11 or n s u d (Geës' Bay, 10.7, 10 ;35 m. Schlamm und hies).] 
Fr ï hero Funde im Eisfor d:

Schwedische Expeditionen: 1864-1873: Safe Bar, 5,5 his 11, 36 lis $72,54 \mathrm{~m}$ und ohne Tiefenangabe; Skans Bay (Billen Bay), $27 \mathrm{~m}$; Advent Bay, 3,5 bis 9, 27 bis $36 \mathrm{~m}$; Green Bay, 3 bis 27,9 his $36,54,72$ bis $90 \mathrm{~m}$ und ohne Tiefenangabe (Riksmuscum, Stockholm, nach A. Moraxulere) 1890; Lingang, 27 bis 35 m; Safe Bay, 54 bis $72 \mathrm{~m}$; Skans Bay (Billen Bay), 54 bis $72 \mathrm{~m}$; (Green Bay, 35 his $72 \mathrm{~m}$ (Kirsckowstrü3 1892); 1898: Xordarm, $36 \mathrm{mn}$ Lithothamnion; Fjordstamm, 40 his $50 \mathrm{~m}$; 1899: Green 13ay, $110 \mathrm{~m}$; 1900: Coles Bay, 50 und 50 bis $100 \mathrm{~m}$, Stein;

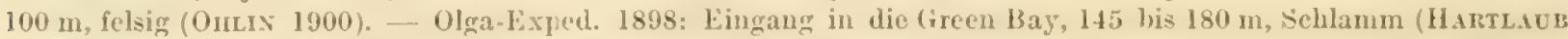
1900). Russ. Exped. 1899 und 1900: Adrent Bay, 9 m, Steine; $16 \mathrm{~m}$, Kies (Brota 1907). Michacl-Sars-Exped. 1901: Green Bay, $150 \mathrm{~m}$ (WoLdEIBK 1908). Belgica-Exped. 1905: (ireen Bay (Grieg 1909).

Wir fanden Sclerocrangon boreas an 44 Stationen, also ungefähr ebenso oft wie Spirontocaris gaimardii und polaris (in Wirklichkeit diirften diese Arten eher allgemeiner sein; in der von $S$. boreas bewohnten Zone wurden zahlreiche Dredgungen ausgeführt). Die Art tritt oft in grosser Individuenzahl auf; zusammen fanden wir etwa 590 Exemplare.

S. boreas lebt im Eisfjord an so gut wie jeder Art von B o d e n. Wir fanden ihn zwölfmal an ungemischtem Schlammgrund, in der Regel allerdings nur in vereinzelten kleinen Exemplaren, in etwas grösserer Individuenzahl nur an einer Stelle mit zähem Schlammgrund (St. 127) und an zwei Orten (St.67, 130), wo der Schlamm stark mit Algenresten gemischt war. Auf mit Stein und Kies gemischtem Schlammgrund fanden wir ihn häufiger (14 Stationen), mehrmals in bedentender Individuenzahl (St. 6,92, 44, 95, 71). An reinem Steingrund ohne Algen oder Balanus fanden wir die Art zweimal; da dieser Boden sehr spärlich vorkommt, hätte man eine grössere Anzahl von Fundorten nicht erwarten können. An mit Laminarien bewachsenem Steingrund fanden wir sie zweimal; da an verhältnismässig vielen solchen Stellen gedredgt wurde, muss sie dort selten sein. Weitaus am häufigsten ist $S$. boreas unter den Balanus porcatus-Kolonien. Wir fanden ihn bei a 11 e n (7) auf solchem Grund vorgenommenen Dredgungen, in der Regel in bedeutender Individuenzahl (insgesamt 210 Exemplare). Häufig ist er auch an Lithothamnion-Grund (7 Fundorte unter 9 Stationen mit wenigstens teilweise strauchförmigem Lithothamnion); hier fanden wir jedoch nur einmal eine grössere Anzahl von Exemplaren (St. 49, etwa $100 \mathrm{Ex}$.).

Die vertikale Verbreitung (s. die nachstehende Übersicht) erstreckt sich im Eisfjord von $2 \mathrm{~m}$ (St. 38, 67) bis $230 \mathrm{~m}$ oder etwas mehr (St. 43). Entschieden am häufigsten ist die Art zwischen etwa 15 und $185 \mathrm{~m}$; wir fanden sie hier fast an der Hälfte (etwa $45 \%$ ) der untersuchten Stationen. Soweit man aus unseren Beobachtungen ersehen kann, ist sie ungefähr gleich häufig in allen Teilen dieses Gebietes; die an dem Schema sichtbaren Ungleichmässigkeiten, vor allem das Fehlen zwischen $71 \mathrm{~m}$ (St. 69) und etwa $100 \mathrm{~m}$ (St. 47) sind der Bodenbeschaffenheit zuzuschreiben.

In der Uferzone, von 2 bis etwa $15 \mathrm{~m}$, fanden wir $S$. boreas an 6 oder 7 Stellen, meist in wenigen kleinen Exemplaren; da wenigstens 25 Stationen in dieser Tiefe untersucht wurden, muss die Art dort weniger häufig als in grösserer Tiefe sein. Doch ist sie auch hier nicht gerade selten, und sie kann in grosser Individuenzahl auftreten. - Unterhalb von $190 \mathrm{~m}$ fanden wir nur einmal ein einziges Exemplar.

Die Temperaturschwankungen der Fundorte bewegten sich zwischen - 0,93 und +5 oder etwas mehr (s. die nachstehende Ubersicht). Das Fehlen in dem kältesten Wasser des Fjordes kamm man nicht einem unginstigen Einfluss der Tempera- 


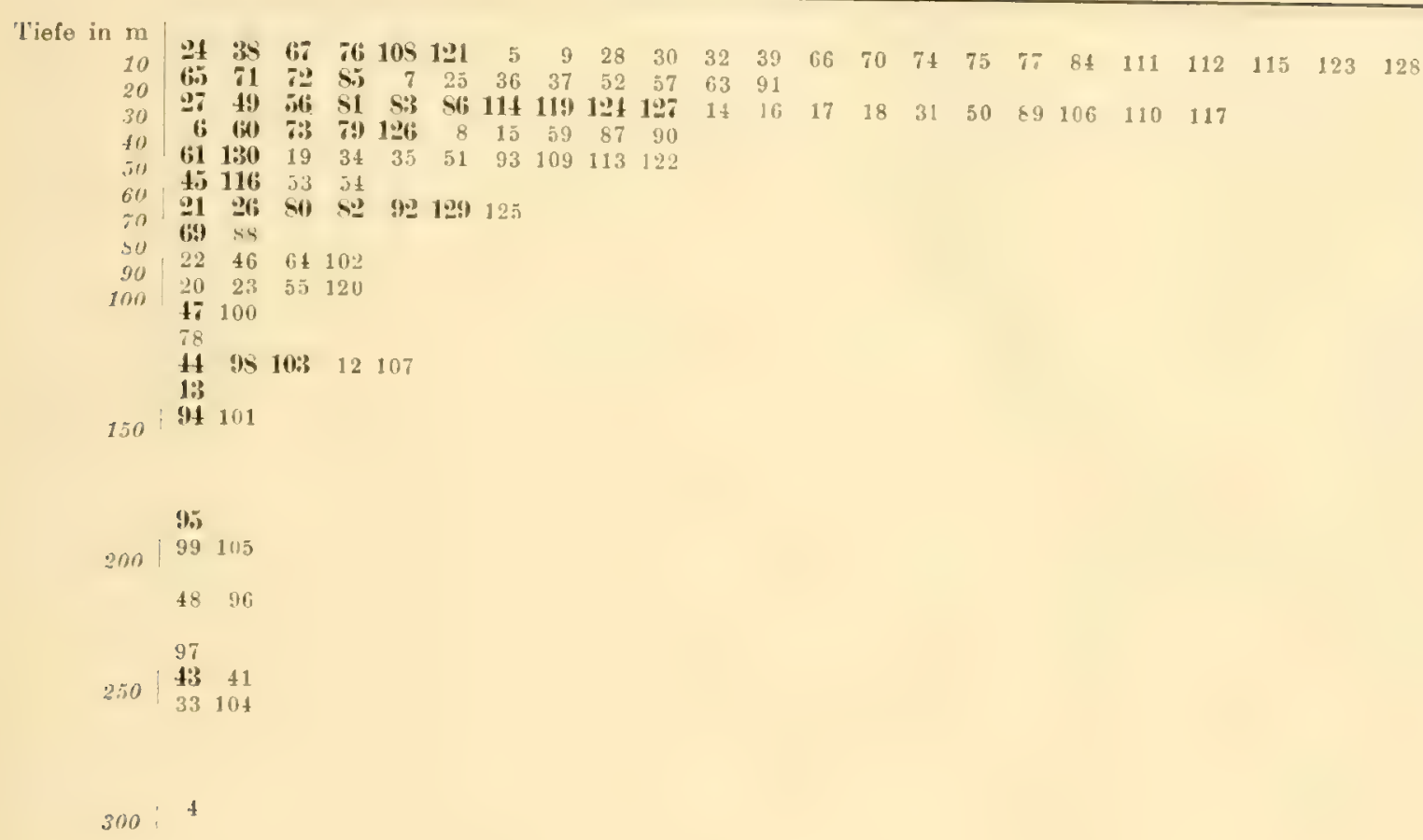

$400 \quad 42$

tur zuschreiben, da die Art in ausgesprochen hocharktischen Gegenden häufig ist. Das eigentiimliche Fehlen in der untersten Reihe des Schemas muss daher durch andere Umstände bedingt sein, zweifellos teils durch die ungünstige Bodenbeschaffenheit (sehr loser Schlammgrund), teils durch die Lage nahe am Gletscherrand.

$+4,3-+5,6^{\circ}$
$+3-+1$
$+2-+3$
$+1-+1,9^{\circ}$
$0-+0,0^{\circ}$
$-0,9--0,1^{\circ}$
$-1,6--1,0^{\circ}$

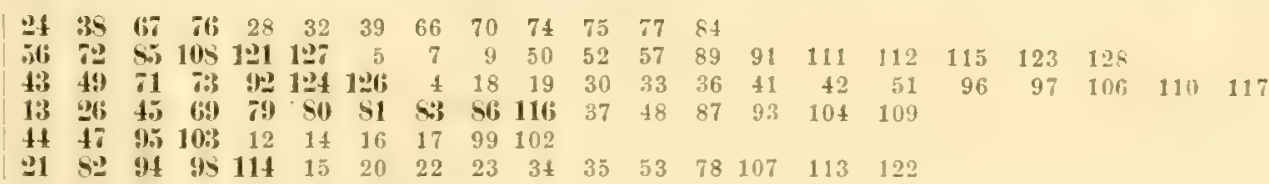

Das Schema zeigt ferner, dass die Art verhältnismässig am häufigsten in Wasser von +1 bis $+2^{\circ}$ Temperatur gefunden wurde (an etwa ${ }^{2} / 3$, in wärmerem Wasser etwa $1 / 3$ der untersuchten Stationen). Noch im wärmsten Oberflächenwasser fanden wir sie jedoch stellenweise häufig, und wenn sie auch möglicherweise etwas kälteres Wasser bevorzugt, muss man unter allen. Umständen zugeben, dass diese Art in Wasser von +3 bis $+5^{\circ}$ Sommertemperatur gut gedeiht und sich also in ihrer Thermopathie deutlich sowohl von den meisten rein arktischen Arten wie von Spirontocaris polaris u. a. unterscheidet. Dass $S$. boreas wirklich verhältnismässig eurytherm ist, wird auch durch einige Tatsachen der allgemeinen Verbreitung erwiesen (s. näher im Allgemeinen 'l'eil). 
Grösse, Fortpflanzung, Entwicklung. Das grösste Exemplar (ein steriles Weibchen) hatte die bedeutende Länge von $113 \mathrm{~mm}$; OHLIN (1895, 1901) gibt eine Maximallänge von 102 bzw. $90 \mathrm{~mm}$, Birula $96 \mathrm{~mm}$ an; Hansen (1908) hat jedoch ein $137 \mathrm{~mm}$ langes Exemplar gesehen.

Eiertragende Weibchen (zusammen 5 Lixemplare) wurden bloss an drei Stationen gefangen; sonst fanden wir nur jugendliche Weibchen. Die weibliche Geschlechtsreife scheint, wie auch meine Beobachtungen an den von der Kolthoff-Lxpedition gesammelten Exemplaren zeigen (vgl. ferner die Angaben von BIRULA 1907; eiertragende Weibehen 95, 94 und $90 \mathrm{~mm}$ ), erst bei einer Körperlänge von $90 \mathrm{~mm}$ einzutreten; in unserem Material findet sich in der Tat nur ein Exemplar von dieser oder noch beträchtlicherer Grösse, das nicht Eier trägt.

Zur Beurteilung der Frage nach der Fortpflanzungs- und Entwicklungszeit stelle ich meine Beobachtungen an dem von uns und der Kolthoff-Expedition (St. 2: Coles Bay; St. 12: W. von Spitzbergen) gesammelten Material zusammen:

\begin{tabular}{|c|c|c|c|}
\hline $\begin{array}{l}\text { Station } \\
\text { Kolthoff- }\end{array}$ & Datum & $\begin{array}{c}\text { o mit nicht oder } \\
\text { wenig entwickelten } \\
\text { Jiern }\end{array}$ & $\begin{array}{l}\text { o mit weit ent- } \\
\text { wickelten Eiern } \\
\text { (mit Augen) }\end{array}$ \\
\hline Exp. 2 & $11 "-20 / 6$ & 1 & - \\
\hline D 12 & $\%$ & - & $\begin{array}{l}1 \text { (Junge grösstenteils } \\
\text { schon ausgeschliipft) }\end{array}$ \\
\hline 49 & $31 / 1$ & -- & 3 \\
\hline 103 & $17 / 8$ & 1 & - \\
\hline 94 & $21 / \mathrm{h}$ & 1 & - \\
\hline
\end{tabular}

Bei der Spärlichkeit des Materials kann man nur den Schluss wagen, dass eine strenge Gleichzeitigkeit in der Fortpflanzung und Entwicklung nicht vorhanden ist; die Entwicklung geschieht teils in der Zeit September bis Juni (St. 103, 94), teils im Sommer (wenigstens St. 49); das erstere ist auch im nördlichen Norwegen der Fall, wo NondGAARD (1905) Anfang April weit entwickelte Eier beobachtet hat.

\section{Allgemeine Verbreitung.}

(Fig. 7.)

$$
\text { Spitzbergongebiet: }
$$

Westspitz hergon: SW. rom Südkap, Hornsund, Belsund, Eisfjord, W. und N. von l'r. Charles l'oreland (Krörer 1843, Sars 1886, Kinckowström 1892, Ihatlaub 1900, OHLin 1901, BHiula 1907, Dons 1915

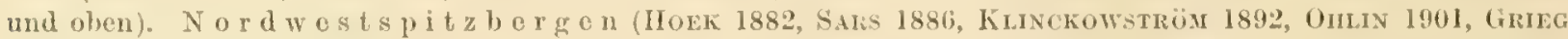

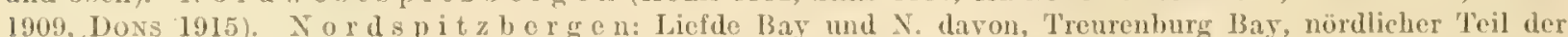
IHinlopenstrasse, Low Island und olno nähero Angaben (l'HP's 1774, Ross 1828, Miers 1877, Kuxckowströs

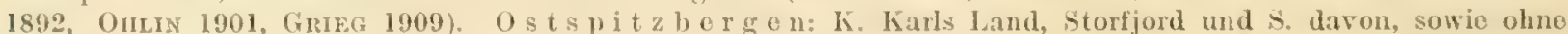
nähere Angaben (Dofleis 1900, Hartuau 13 1900, Onlis 1901, Briuxa 1907). Hopen Eiland (Doss 1915). Zwischen

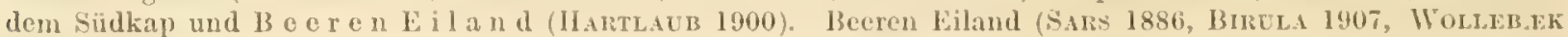
1908).

Dio oben angeführten Bcobachtungen crlauben den sicheren Schluss, dass Sclerocangon boreas im ganzen Spitzbergengebiet sehr häufig ist. Nach der Aufassung Dorcesw's würde er sich wie die Spirontocaris.Arten verhalten ( mobgleich nicht ganz so streng») und also in Westspitzhergen nur in kaltem Wasser, vorwiegend in grösserer Tiefo, loben. Dieso Ausicht ist, wio das über dio Verbreitung im Visfjord Gesagto zeigt, ganz irrig. 
Übrigearkisehe und boreoaktiseheregion:

Karisehes Meer, südlieher Teil (RUiJs 1887; dio Angahe von BHula [1907] und Stemmevsw [1912, 1913], dass das Vorkommen in Karischen Meer bisher nicht festgestellt worden sei, ist also unrichtig). Jugor sehar, TaigatschInsel, Süd- und Westküste von Noraja Semlja, Matotschkin schar, Barentsmeer, Franz Josephs Land (D)'UniBax 1880, Miers 1881, Holk 1882, Stuxbeig 1886, Hinsen 1887 a, RuiJs 1887, Scott 1899, Knipowitscit 1901, Scott 1899, Dons 1915). Weisses Meer, Hurmankïste (JArzynsky 1885, Pferreir 1890, Stierex 1895, Brrula 1897, 1899, Doflein 1900, DerJugin 1906, 1912, Awerinzew 1909). Ostfinmmarken (Dantelssen 1861, Norman 1902, Nordgated 1905, Wollebar 1908, Dons 1915; ferner II. Sars 1858, G. O. Sars 1886: Finninarken). Kalte Fjordo in Westfinnmarken und der Lofotengegend (s. unten). - O. von Island, Ost- und Nordküsto von Island (IIANSEN 1908, ferner ScImidt 1904, APPELlör 1906, 1912 [गboreoarktische Region Islands »]). Jan Mayen (KoeLBeL 1886). — Nordost-, Südost, West- und Nordwestgrönland (STEPIENsen 1913,1916 und frühero Autoren). - Ellesmero Iand

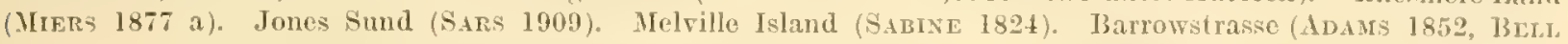

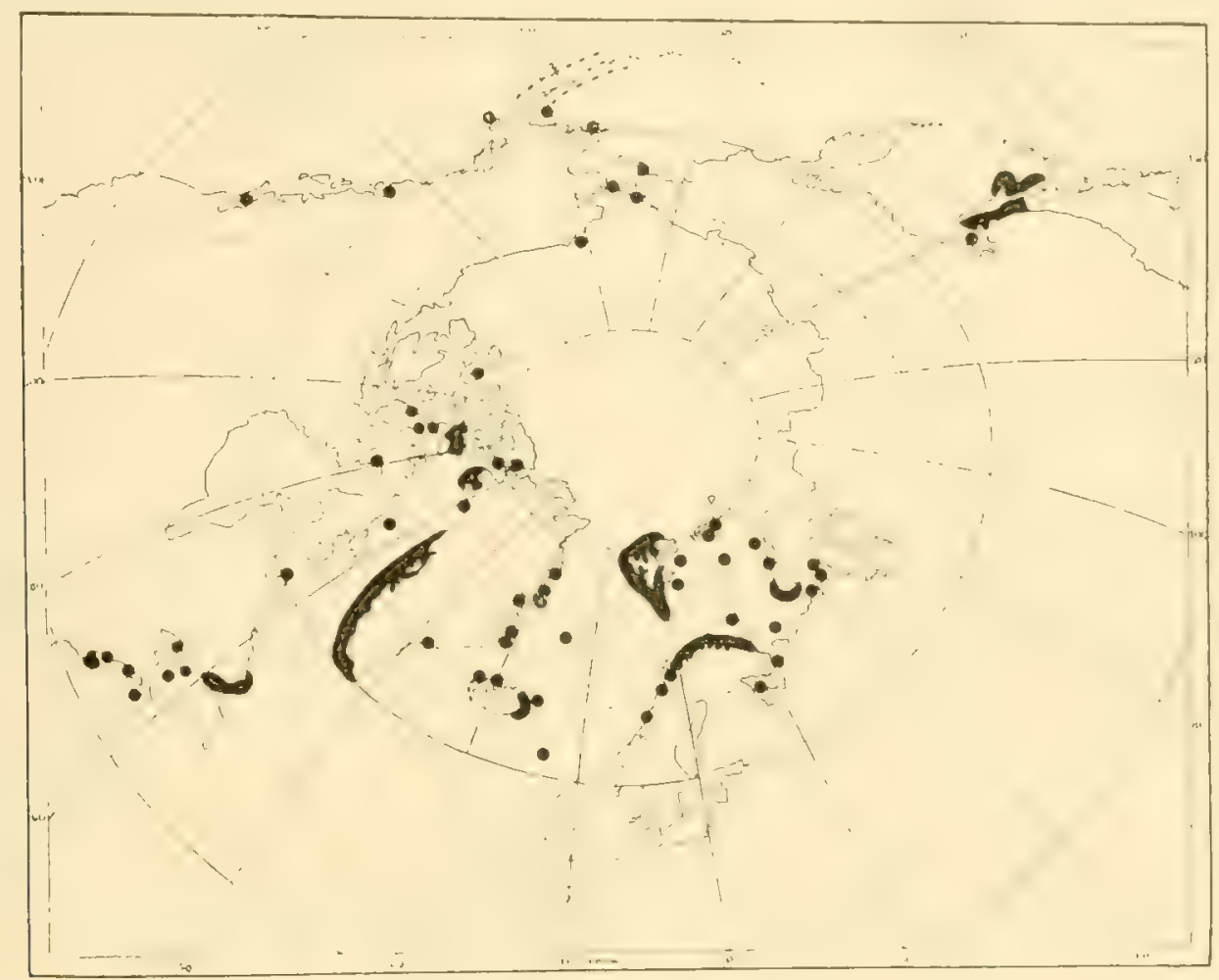

Fig. 7. Sclerocrangon boreas.

1855). Melville-Halbinsel (Ross 1835). P’r. Regents Insel (Ross 1826). Westküsto ron Davis Strait (F.IBrs: 1824). Ifudson Strait, üstlicher Lingang (Wintesves 190I). - Nordostküste von Labrador, Golf von St. Law-

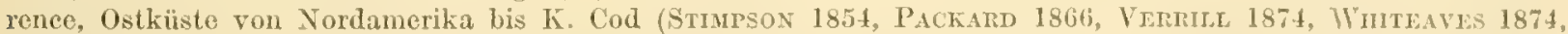

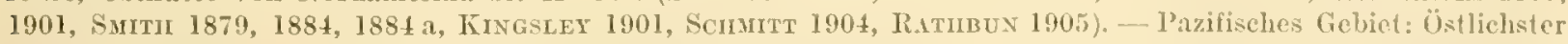
Teil der Nordküste ron Sibirien (STUXBERG 1882) [fermer BRANDT 1851: 》Lismeer» (sibirisches?)]. Nordküsto von Alaska, Beringsstrasse (und Eismeer olme Lokalangaben: Stmpsos), Beringsucer (Stmursos 1860, Murdocir 1885, besonders RatiBun 1904). Ochotskisches Meer und nürdlicher 'leil des 'l'ataren-(iolfes (IBreaxid 1851, BRASINIKOW 1907, BaLSS 1914).

$$
\text { Borealo Region: }
$$

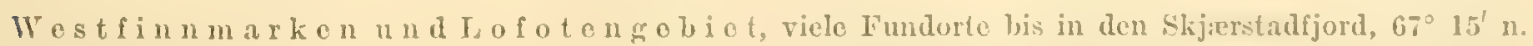

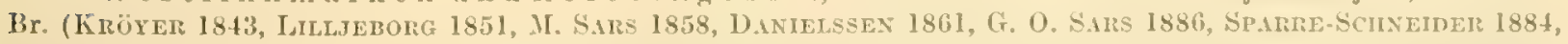

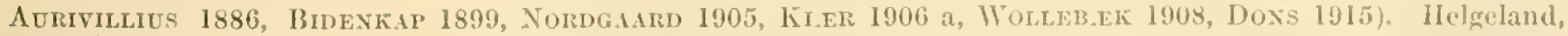
$68^{\circ} 50^{\prime} \mathrm{n}$. Br. (Doss 1915). Viele, wahrschoinlich die meisten der Fundorte liegen in den kalten, boreoarktisehen Fjorden, andere Fumde (bei Grötö usw.) zeigen jedoch, dass dio Art auch unter borealen IBedingungen vorkommen

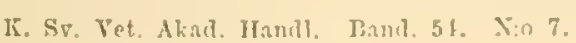


kann. Nach M. SArs $(1858,1861$ a) wäre sie selten bis Bergen rerbreitet, diese Angabe dürfte jedoch nieht rich. tig sein; nach den Erfahrungen aller späteren Forseher ist sie nur in der nächsten Näle der boreoarktischen Ge. genden zu finden.

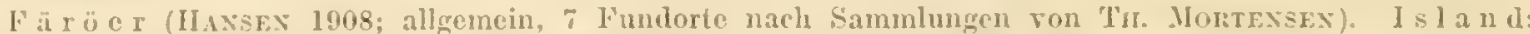
Südostkïste (WoLterxk 1908); Sïdwest- und Westkïste (IIAses 1908).

Verbreitung im wamen 'Toil dos paziseheng Gietos: Aleuten bis Cross Sound, Siblostalaska (Ratibux 1904); Georcia-Strasse (Ifaxz 1901).

Bat hy metrische Texbreitu $\mathrm{g}$. In der arlischen liegion erstreckt sich die bathymetrische Verbreitung von $2 \mathrm{~m}$ (s.oben) bis $315 \mathrm{~m}$ (STEPHFNSEx 1912 a, 191:3) oder vielleicht $360 \mathrm{~m}$ (HAXSEN 1887). Unterhalb von etwa $180 \mathrm{~m}$ ist dic Art iiberall, wie im Eisfjord, selten; in grösserer Tiefe als $200 \mathrm{~m}$ ist sie bisher mit Sicherheit nur sechsmal gefunden worden (einmal von uns, s. ferner HoEK 1852, HANSEA 1857 , WOLAFBAK 1908, STEPHFNSEx 1912 a). Die wenigen Fundorte in der borealen liegion Norwegens liegen in 'Tiefen zwischen 6 bis 24 und $100 \mathrm{~m}$ (NordrAARD). Bei den Färöem lebt die Art mach HANSFN zwischen 2 bis 5,5 und $180 \mathrm{~m}$.

\section{Sabinea septemcarinata (SAB.).}

\section{Verhreitung im Lisfjorll.}

Verzeichnis der Fundorte (s. Karte 4):

\begin{tabular}{|c|c|c|c|c|c|c|c|c|c|}
\hline $\begin{array}{l}\text { Nr. } \\
\text { der } \\
\text { Stat. }\end{array}$ & Ort und & l Dat & um & Tiefo & $\begin{array}{l}\text { Wnsser- } \\
\text { temperatur }\end{array}$ & $\left\{\begin{array}{l}\text { Solz- } \\
\text { gelialt } \\
\% \text { no }\end{array}\right.$ & Bodenbeschaffenheit & Gerät & $\begin{array}{l}\text { Anzałl und } \\
\text { Grösso }\end{array}$ \\
\hline 42 & Srensksunds & sticfo & .24 .7 & $406-395 \mathrm{~m}$ & $382 \mathrm{~m}:+2,61^{\circ}$ & $3 \$, 90$ & Loser Schlamn & Trawl & $19 \mathrm{Ex}, 24-$ \\
\hline 4 & $x$ & & .15 .7 & $27-313 \mathrm{~m}$ & {$[$ Li:wn+2.5 } & - & Schlamm & Ottertrawl & $\begin{array}{l}\text { 2) Ex, } 52,75 \\
\text { min }\end{array}$ \\
\hline 12 & Safe IBay & . . & .016 .7 & $118-127 \mathrm{~m}$ & $108 \mathrm{~m}:+0,65^{\circ}$ & 34,43 & Loser Schlamm & Trawl & Etwa 15 Ex. \\
\hline 15 & $>\quad$. & . . . & . . & $33 \mathrm{~m}$ & $30 \mathrm{~m}:-0,59^{\circ}$ & 34,16 & " n & 1kl. Dredgo & $1 \mathrm{Ex}, 25 \mathrm{~mm}$ \\
\hline 20 & Yimer I3ay. & . . &. .20 .7 & $85-100 \mathrm{~m}$ & $85 \mathrm{~m}:-0,2 \mathrm{~s}^{\circ}$ & 34,54 & $\begin{array}{l}\text { Sohr loser Schlnmm, stel- } \\
\text { lenweise Sitein mit } \\
\text { Algen }\end{array}$ & Trawl & $\begin{array}{l}5 \mathrm{Fx}, 24- \\
36 \mathrm{~mm}\end{array}$ \\
\hline 33 & Fjordstamm & a . & .23 .7 & $263-256 \mathrm{~m}$ & {$\left[+2 \text { bis }+2,6^{\circ}\right]^{\text {vul }}$} & - & Loser Schlamm & 2 & $\begin{array}{l}2.2 \mathrm{Ex}, 25- \\
76 \mathrm{~mm}(29 \\
\text { ovig. })\end{array}$ \\
\hline 41 & $"$ & . . &. .24 .7 & $234-254 \mathrm{~m}$ & $251 \mathrm{~m}:+2.56^{\circ}$ & 34,96 & , & " & $17 \mathrm{Ex.,} 24$ \\
\hline 21 & $\begin{array}{l}\text { Eingang in d } \\
\text { Bay. . . }\end{array}$ & die'Tn & indra & $71-68 \mathrm{~m}$ & $-0,93^{\circ}$ & 34,29 & $\begin{array}{l}\text { Sehr loser Schlamm, stel- } \\
\text { lenweise Stein }\end{array}$ & n & $\begin{array}{l}16 \mathbf{E x}, 32- \\
69 \mathrm{~mm}\end{array}$ \\
\hline 94 & Fjordstamm & $n$. & .21 .8 & $147-141 \mathrm{~m}$ & $140 \mathrm{~m}:-0,62^{\circ}$ & 34,49 & $\begin{array}{l}\text { Loser Schlamm mit klei. } \\
\text { nen Steinen }\end{array}$ & " & $\begin{array}{l}11 \mathrm{Fx}, 37- \\
78 \mathrm{~mm}\end{array}$ \\
\hline 92 & Nordarm & . . &. .19 .8 & $85-45 \mathrm{~m}$ & $42 m:+2,02^{\circ}$ & - & $\begin{array}{l}\text { Loser Schlamm mit Kies } \\
\text { und Sand. Am Ende } \\
\text { der Dredgung Steine } \\
\text { und Iamminarien }\end{array}$ & 2 & $\begin{array}{l}29 \text { kx., } 33- \\
\text { 8. } \mathrm{mm} \text { ( } 2 \\
\text { ovig.) }\end{array}$ \\
\hline 98 & \% & . . . &. .27 .8 & $130-116 \mathrm{~m}$ & $115 \mathrm{~m}:-0.82^{\circ}$ & 34,40 & Toser Schlnmm & , & $\begin{array}{l}+\mathrm{Ex} \cdot, 38- \\
67 \mathrm{~mm}\end{array}$ \\
\hline 99 & , & . . . & . . y & $197-190 \mathrm{~m}$ & $190 \mathrm{~m}:+0.80^{\circ}$ & 34.72 & $"$ & , & $\begin{array}{l}13 \mathrm{Hx}, 36- \\
82 \mathrm{~mm}(1 \text { f } \\
\text { ovig.) }\end{array}$ \\
\hline 102 & $\begin{array}{l}\text { Jordarm. } \\
\text { in die Yol }\end{array}$ & $\begin{array}{l}\text { Jing } \\
\text { lain } 1\end{array}$ & $\begin{array}{l}\text { gang } \\
\text { lany } 14.4\end{array}$ & $70-93 \mathrm{~m}$ & $85 \mathrm{~m}:+0,6 \mathrm{~s}^{\circ}$ & 34,25 & $\begin{array}{l}\text { Zither und festerSchlamm } \\
\text { mit vielen Steinen }\end{array}$ & , & $1 \mathrm{Ex}, 3: 3 \mathrm{~mm}$ \\
\hline
\end{tabular}




Nr.
$\begin{aligned} & \text { der } \\ & \text { Stat. }\end{aligned}$

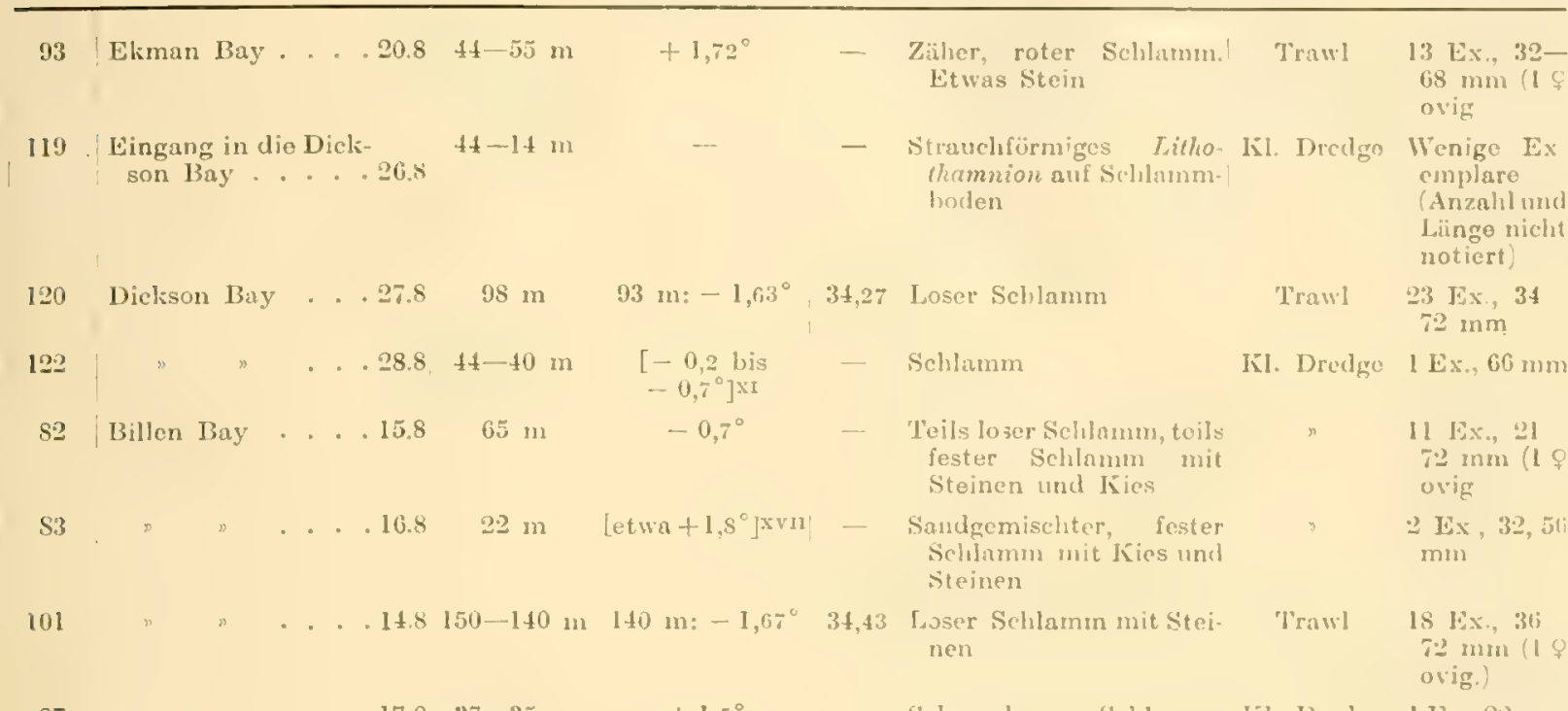

43

I 50 Tempel Bay . . . 29.7 25 m $\quad\left[+3 \text { bis }+4^{\circ}\right]^{x x}$

56 Tempel Bay, Bjonas $30 \mathrm{~m}+3,78^{\circ}$

Hafen .... . 31.7

47

Eingang in die Sissen

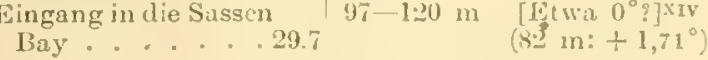

48

Ostarm … $31.7199-230^{2} 210 \mathrm{~m}:+1,27^{\circ} 34,72$

101 Fjordstamm ... 17.s $260 \mathrm{~m}, 270 \mathrm{m:}+1,62^{\circ} \quad 34,79$

41 Eingang in die Ad- $150-110 \mathrm{~m}: 128 \mathrm{~m}:+0,01^{\circ} 34,54$ Loser Schlamm mit Lies vent Bay -...27.7

45
Advent Bay . . 28.7 $70-42 \mathrm{~m} \quad 41 \mathrm{~m}:+1,85^{\circ} \quad 34,1 \mathrm{~s} \begin{gathered}\text { Lo:er, aber zälier } \\ \text { Schlamm }\end{gathered}$

Fjordstamm . . 21.8/188-181 m $\left[0 \text { bis }+0,5^{\circ}\right]^{\text {vi }}-$ Schlamm mit Steinen
Stein, Ifies und Schalen

mit Lithothamnion

Zäher Schlamm

$$
\text { Steineu }
$$

Loser Schlamm

$$
\text { (n) }
$$

etwus líies 


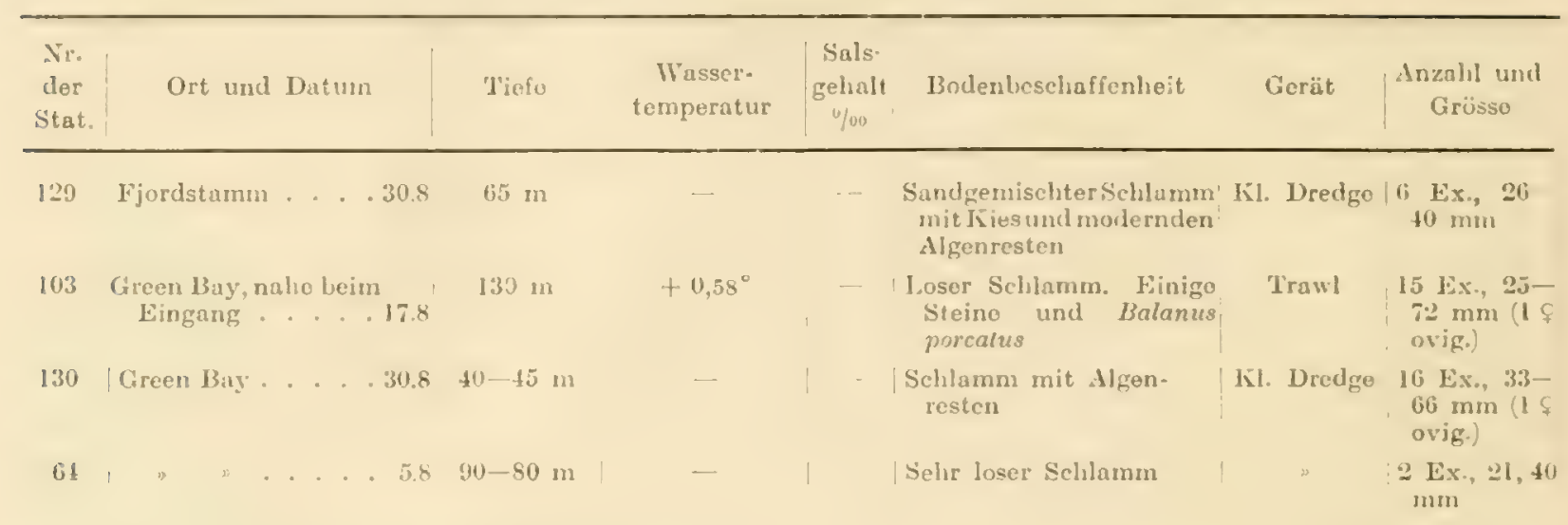

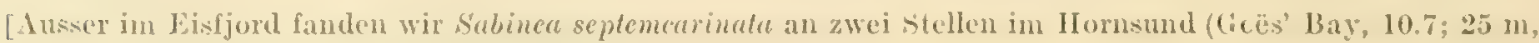
Schlamm mit etwas Kies; $10-35 \mathrm{~m}$, Schlarmm und Kies).]

Fr i li ere Funde in E is f jord:

Schwed. Exjed. 1868: Adrent Bay, 36 bis $54 \mathrm{~m}$; Green 13ar, 9 his 36 m; 1873: Skans Bay (Billen Bay), 27 u (Rilismuseum, Stockholm). Mrers 1877: Green Bay ohno Tiefenangabe. Helgoland-Exped. 1898: F'jordstanm, 210 bis $240 \mathrm{~m}$, Schlamm mit steinen (Dorleis 1900). Schwed. Fxped. 1900: Coles Bay, 50 his $100 \mathrm{~m}$, Stein (OnLIN 1901). IRuss. Exped. 1900: F'jordstamm, 205 m, T'emp. - 0,8 , 243 m, -0, $8^{\circ}$, Schlamm und Grus; Advent Bay, 7 his $9 \mathrm{~m}$, Crus; Billen Bar, 142 his $133 \mathrm{~m},-1,9^{\circ}$, Schlamm mit Steinen (Brnula 1907). Michael sars-Exped. 1901: Green Bay, $150 \mathrm{~m}$ (Woldeb.X. 1908; s. auch Coldett 1905; im Magen ron Liparis liparis); Eisfjord olne nähere Angaben, $140 \mathrm{~m},+1.1^{\circ}$ (vermutlich der von WOLLEB.E erwähnte Fundort) (APPEL_ör 1906).

Wir fanden Sabinea septemcurinata an 40 Stationen. Sie ist also im Eisford äusserst häufig, in Wirklichlieit zweifellos häufiger als die an 44 Stationen gefundene Art Sclerocrangon boreas; die geringere Anzahl der tundorte beruht darauf, dass sie nicht so häufig in den geringeren 'Tiefen ist, wo wir' die mêisten Dredgungen ausführten. Die Individuenzahl (insgesamt etwa 490 Exemplare) war stellenweise bedentend.

Diese Art scheint mehr als irgend eine andere Decapodenart des Eisfjords (ausser etwa Pandalus boreatis) von der $B$ od e $n$ be s e ha f f e n h e it abhängig zu sein und zwar lebt sie so gut wie ausschliesslich auf Schlammboden. Von unseren 40 Fundorten hatten 19 reinen (in der Regel losen) Schlammgrund; an 17 bestand der Boden aus mit Steinen oder Kies gemischtem Schlamm oder war stellenweise schlammig, stellenweise steinig. Nur 4 Stationen hatten härteren Boden, bestehend aus Kies, Stein und Schalen mit mehr oder weniger Lithothamnion (St. 49, 69), aus Lithothamnion auf Schlammgrund (St. 119 ) oder aus Balanus-Kolonien (St. 126); an diesen Stellen wurden nur wenige Exemplare erbeutet.

Die bathymetrische Verbreit ung (s. die nachstehende Übersicht) crstreckt sich von $15 \mathrm{~m}$ bis in die grösste Tiefe des Fjords. Tm oberen Teil dieses beträchtlichen vertikalen Verbreitungsgebietes, bis zu 30 oder wahrscheinlich $40 \mathrm{~m}$ hinab, ist die Art jedoch selten; wir fanden sie hier nur an wenigen Stellen, jedesmal nur in wenigen kleinen Exemplaren. Wir arbeiteten in diesen Tiefen nur mit kleinen Dredgen, die ja stets eine verhältnismässig geringe Anzahl von grösseren Crustaceen fangen, ein Vergleich mit andern Arten, vor allem Sclerocrangon boreas, zeigt jedoch, dass die Art unzweidentig viel seltener ist als in grösserer Tiefe. Das Fehlen in der eigentlichen Uferzone und die Seltenheit in der darunter folgenden Schicht kamn nicht durch den Mangel 
an geeignetem Grund hervorgerufen sein, denn an zahlreichen der dort untersuehten Stationen bestand der Boden aus reinem oder mit Steinen gemisehtem Schlamm.

Zwischen 40 und 60 oder $65 \mathrm{~m}$ ist die Art bedentend häufiger, und hier wurden zahlreichere, teilweise geschlechtsreife Exemplare erbentet; das Schema zeigt jedoch, dass sie hier noch nicht so allgemein ist, wie in noch grösseren Tiefen. Von etwa $65 \mathrm{~m}$ an wurde sie an der Mehrzahl der untersuchten Stationen gefangen (26 liundorte unter 41 Stationen). Ob die Verbreitung innerhalb dieses Gebietes ganz gleichmässig ist oder nicht,

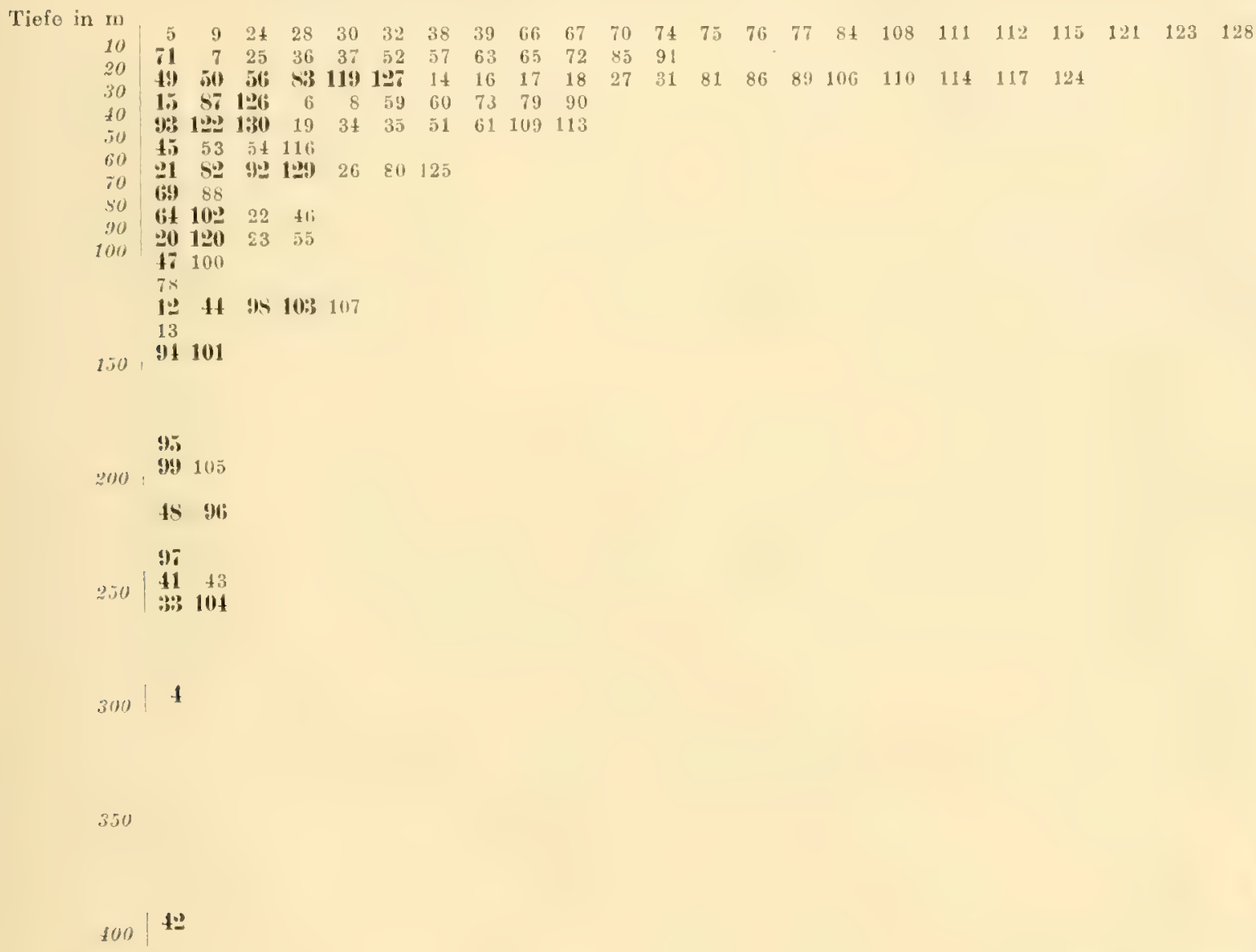

kann nicht entschieden werden. In den grösseren Tiefen, von etwa $120 \mathrm{~m}$ an, wird die Art eher noch allgemeiner als seltener; bei den in grösserer Tiefe als $200 \mathrm{~m}$ ausgefuihrten Dredgungen fehlte sie sogar nur einmal. Doch ist es nicht unwahrscheinlich, dass sic unterhalb von $300 \mathrm{~m}$ wiederum weniger häufig ist, denn in solcher 'Tiefe war sie früher nur ein einziges Mal gefunden worden.

Die Temperaturen der Fundorte schwankten zwischen etwas mehr als $+3^{\circ}$ und $-1,67^{\circ}$. Die Verteilung der Fundorte auf die zwischen diesen Lxtremen licgenden Temperaturen wird durch die nachstehende Ủbersicht erläutert. Wan sicht daraus zunächst, dass die Art in Wasser von hoher negativer und niedrig positiver 'Temperatar $(-0,9$ bis $+2,6$ oder +3$)$ sehr häufig ist. Im kältesten Wasser fanden wir sie nur zweimal, jedesmal aber in zahlreichen, teilweise gesehlechtsreifen lixemplaren; das 
Fichlen an den übrigen Stationen muss wahrscheinlich auf die Bodenbeschaffenheit oder die Nahrungsverhältnisse zuriickgefihrt werden (St. 54, 55, 125: loser roter Schlammgrund; St. 88, 100: nahe am Gletscherrande gelegen).

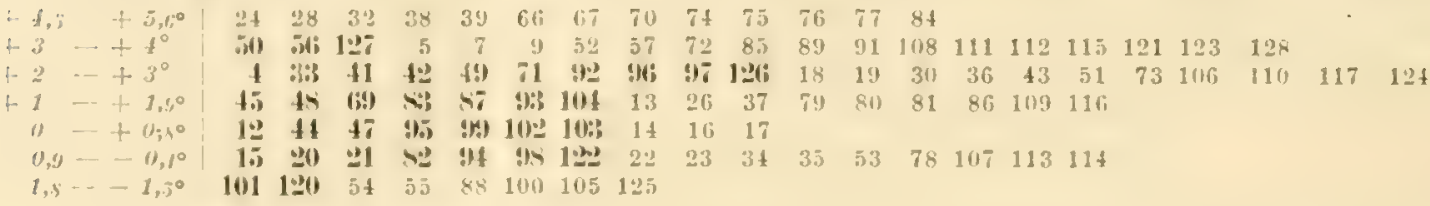

Dagegen ist es gegenwärtig unmöglich zu entseheiden, ob die obere 'Temperaturgrenze im Eisfjord wirklich oder seheinbar ist. In geringerer Tiefe als $15 \mathrm{~m}$ fehlt die Art nämlich iiberall so gut wie ganz. Aus der vertikalen Verbreitung in verschiedenen Gegenden kann man also nicht ersehen, ob die obere Temperaturgrenze bei etwa $+3{ }^{\circ}$ oder höher liegt. Eine wesentlich höhere Temperatur als etwa +5 erträgt die Art, wie dic ganze Verbreitung zeigt, zweifellos nicht; vielleicht erträgt sie ein Sommermaximum von etwa $+5^{\circ}$, wahrscheinlich jedoch nur ziemlich voribergehend; sonst wïrde die Verbreitung im nordwestlichen Norwegen wohl kaum so streng an die kalten Fjorde gebunden sein.

Fortpflanzung, Entwicklung. Wiertragende Weibchen finden sich von 11 Stationen. Ihre Grösse beträgt $63-84 \mathrm{~mm}$; in der Regel scheint die weibliche Geschlechtsreife erst bei einer Länge von 66 bis $68 \mathrm{~mm}$ einzutreten. Úber die Laichzeit und die lintwieklungszeit der Eier lässt sich trotz des verhältnismässig grossen Materials wenig Bestimmtes sagen: die nachstehende Ubersicht bericksichtigt auch die Funde der Kolthoff-Lxpedition in der Coles Bay Juni 1900 (3 Stationen; OnLIs erwähnt nur eine davon):

\begin{tabular}{|c|c|c|c|}
\hline $\begin{array}{l}\text { Station } \\
\text { Folth. }\end{array}$ & Datum & $\begin{array}{c}\text { mit nicht oder } \\
\text { wenig entwickelten } \\
\text { Eiern }\end{array}$ & $\begin{array}{l}\text { o mit weit ent- } \\
\text { wickelten Eiern } \\
\text { (mir Augen) }\end{array}$ \\
\hline Exp., 3 St. & $25-22 / 6$ & 4 & - \\
\hline 33 & $23 / 5$ & 1 & 1 \\
\hline 44 & $3 i / 8$ & - & 1 \\
\hline 17 & $2 ! 1 / i$ & 10 & 1 \\
\hline 101 & $14 / 8$ & - & 1 \\
\hline 82 & $25 / 8$ & 1 & \\
\hline $10: 3$ & $1 \% / 8$ & 1 & \\
\hline 92 & $19 / 8$ & $\because$ & . \\
\hline 93 & $=20 / 8$ & 1 & 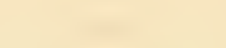 \\
\hline 95 & $=1 / 8$ & 1 & 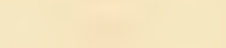 \\
\hline 99 & $27 / 8$ & - & 1 \\
\hline 130 & $30 / 8$ & 1 & \\
\hline
\end{tabular}

Man kann aus diesen Tatsachen nur zwei Schliisse ziehen: 1. Bei zahlreichen Tndividuen findet die Entwicklung im Sommer statt, wahrscheinlich mit Begim im Juli oder bisweilen vielleicht später. 2. Auch im Herbst oder Winter muss jedoch eine Entwicklung vorsichgehen, denn bei den meisten Mitte bis Ende August gefangenen eiertragenden Exemplare waren die Eier noch nicht oder wenig entwickelt. So verhält sich 
die Art auch.im nördlichen Norwegen. Von März bis April sind in dieser Gegend nach WoLLEBAK (1908) a 11 e Eier stark entwickelt, im Juli und August fanden sich nur wenig entwickelte Eier; der norwegische Autor glaubt, dass SS. seplemcarinate zweimal im Jahre laicht, teils im Anfang des Sommers, teils im Herbst. Unsere Befunde können ebensogut durch die Annahme einer sich über eine längere Zeit, vielleicht das ganze Jahr, erstreckenden Fortpflanzungszeit erklärt werden; sie erlauben aber auch die Amnahme von zwei ineinandergreifenden Laichperioden.

\section{Allgemeine Terbreitumg.}

(lig. 8.)

$$
\text { S litzbergengobict: }
$$

Westspitzbergen: Hornsund, Belsund, Eisford (KLInekowström 1892, Omlin 1901, Biruta 1907, DONS 1915 und oben) [ferner KRörer 1843, SARs 1886: Spitzbergen; DorterN 1900: Westspitzhergen]. No r l-

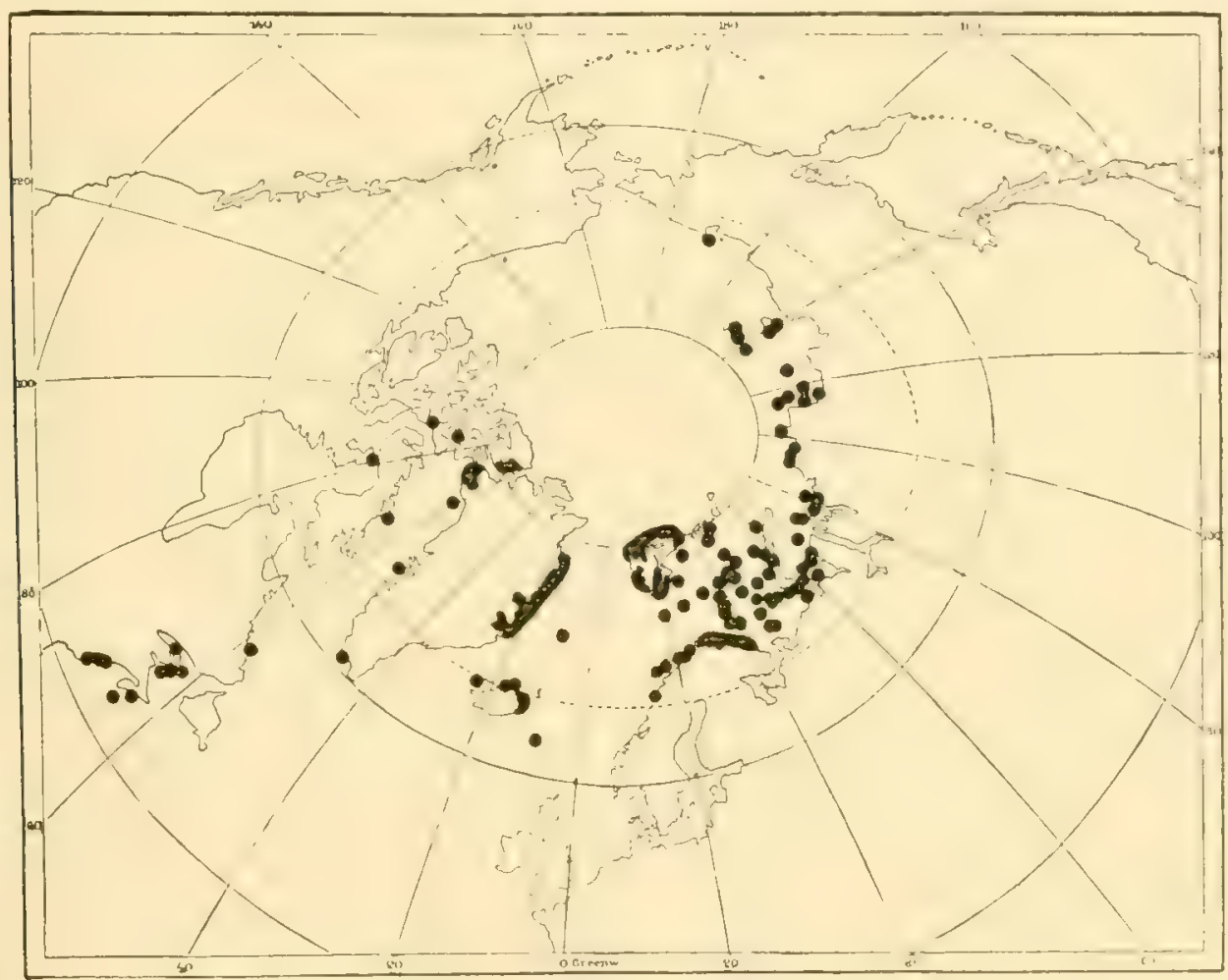

Fig. 8. Sabinea septemcarinata.

westspitzhergen (Dons 1915). Nordspitzbergen: Liefde Bay, Wijde 13ay, N. und No. rom Nord. ostland (Ross 1828, Dorlein 1900, Ondin 1901). Ostspitzbergen: K. Karls Land, Ilopen Eiland, Stor-

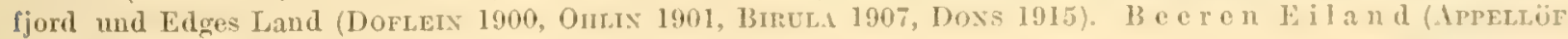
1906, WOLLEB.EK 1908).

Sabinea septemearinata ist, wic aus diesen Angaben hervorgeht, im ganzen (iehiet allgemein; lesonders dio zahlreichen Fundo im Eisfjord zeigen, dass sie nicht, wie DofLEIx meint, porwiegend in Ostspitzbergen zu Hause ist. 


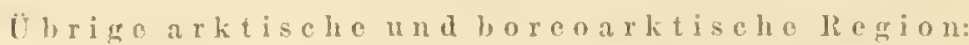

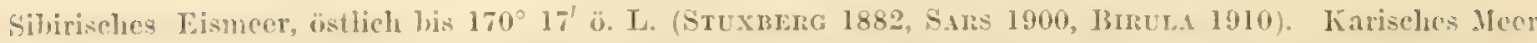
und etwas 0. davon (Stuxierg 1882, 1886, HAssex 1887 a, RuiJs 1887, Biruta 1900, 1910). Karische l'forte, sü̈l- und Westküste ron Noraja Semlja, Franz Josephs Land und ganzes Barentsmeer (1) Urisł 1880, HoEK 1882.

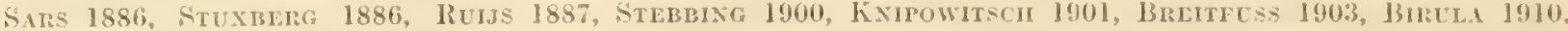

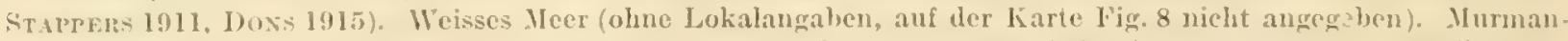

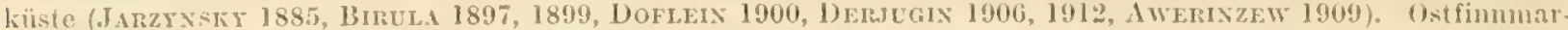

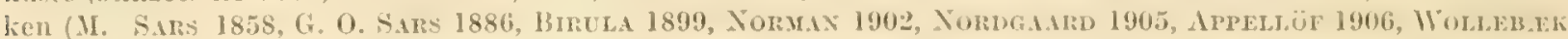
1908, Doss 1915). Kalte Fjorde in nordwestliehen forwegen (s, unten). - Färö-Island-Rüeken (WoLLEB.ri

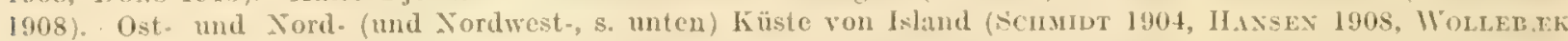

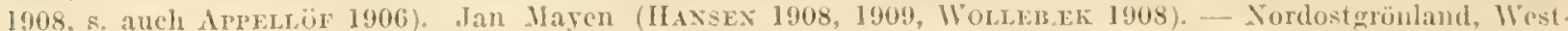
grönland (nur 3 Fundorio), Melville Bay, Nordwestgrönland (STErnExsex 1913,1916 und frühere Autoren). - Elles. mere Land (MLers 1877 a). Jones Sund (SARS 1909). Barrow Strasse (ADiMs 1852). Melville-IIalbinsel (Ros: 1835). Ostkïsto ron Baffin Land (S.IBINE 1824). - Nordostküste von Labrador, Golf von St. Lawrence (P'ICKAIII

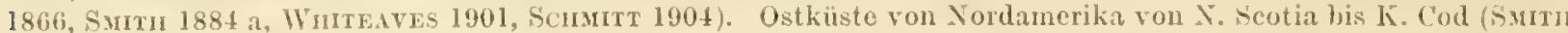

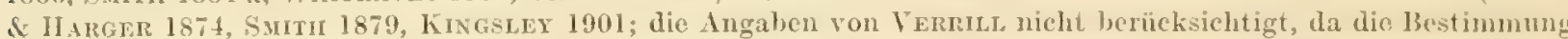
unsicher ist). - Pazifisches Gebiet: Tom Beringsmeer nicht bekannt, dagegen wie es seheint in sihirischen Eis. meer unweit der Beringsstrasso gefunden (Stmpsox 1860: nin Oceano Aretico, prope oras Sibcriaes).

$$
\text { Verbreitung in Tordwest-Norwegen: }
$$

Nach den ältoren norwegischen Autoren lebt S. septemearinata an der norwegischen Vestküste; die Südgrenzo liegt mach II. SArs $(1858,1861,1861$ a) bei Bergen. Diese Angaben sind indessen ganz unsicher, da ihre S. sep.

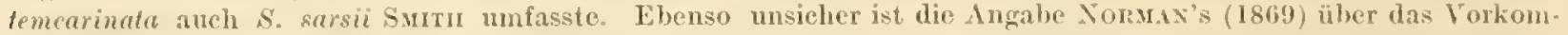
men dieser Art hei den Shetland-Inseln in $150 \mathrm{~m}$ Tiefe; es dürfo sich zweifellos um S. sarsii handeln. Nach (: O. S.Ans (1890), der die Selbständigkeit der beiden Arten annimmt, leben beide an der norwegischen Westküste, nürd. lich bis Lofotenn (hei Finnmarken nur S. septemearinata). Diese Angabe ist sieher unrichtig; licin neuerer For scher hat S. septemearinata südlich rom Saltenfjord gefunden (s. APPELö̈r 1906, Wod.FB EK 1908).

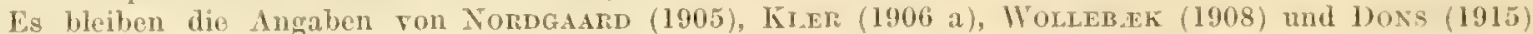
Sie erwähnen S. septemearinate teils aus Ostfinmmarken (s. oben), teils aus zahlreichen Lokalitäten an der nördlichen Testküste, von Westfinnmarken bis an den Saltenfjord (im Kraenangen wurde sio sehon vou SPARRE

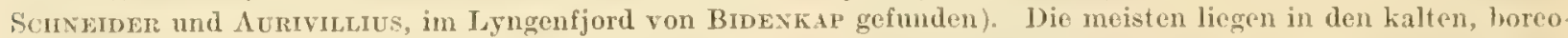
arktischen Fjorden; in einigen Fälen kann man nichts Bestimmtes iiber dio lyydrographischen Verhältnisse sayen, und ganz vereinzelte Fundorte (von DoNs mitgeteilt) liegen wahrscheinlieh im warmen Gehiet. Sicher ist jedenfalls, dass diese Art so gut wio ausschliesslich dem arktischen und boreoarktisehen Gebiet angehört. HAxSEx (1908) crwänt sio von zwei Fjorden in $\mathrm{Y}$ or $\mathrm{d}$ w o s t is la $\mathrm{n}$ d: dieses Gebiet ist wohl ziemlich warm, doch diirften rielleieht anch hier borcoarktischo Bedingungon vorkommen.

Bathymetris che Verbreit ung. Die obere Verbreitungsgrenze liegt bei $9 \mathrm{~m}$ (BIRUlA 1907), die untere bei $450 \mathrm{~m}$ (Wolleb ak 1908). Oberhalb von 15 oder $20 \mathrm{~m}$ sind iiberall nur ganz sporadische Funde gemacht worden, und noch bis in etwa 40 $m$ ist die Art (wenigstens in nicht hocharktischen Gegenden), ebenso selten wie im lisfjord. Nach unten zu ist sie häufig bis in etwa $300 \mathrm{~m}$ 'Tiefe; noch tiefer ist sie bisher nur' zweimal gefunden worden (von uns, ferner von einer Michael Sars-Wxpedition, s. WoLI.EBTEK 1908). 


\section{lupagurus pubescens ( $\left.\mathrm{KR}_{\mathrm{r}}\right)$.}

\section{Verbreitung im Eisfjort.}

Verzeichnis der Fundorte (s. Karte 2):

\begin{tabular}{|c|c|c|c|c|c|c|c|c|c|}
\hline $\begin{array}{l}\text { Nr. } \\
\text { der } \\
\text { Stat. }\end{array}$ & Ort und & Dat & tum & Tiefo & $\begin{array}{l}\text { Wasser- } \\
\text { temperatur }\end{array}$ & $\begin{array}{l}\text { Salz: } \\
\text { gehalt } \\
\% 0\end{array}$ & Bodenbeschaffenheit & Geriit & $\begin{array}{l}\text { Anzahl und } \\
\text { Grösso } \\
\text { (Länge des } \\
\text { Curapax) } \\
\end{array}$ \\
\hline 13 & $\begin{array}{l}\text { Eingang in } 1 \\
\text { Bay. . }\end{array}$ & die $s$ & Safo & $125-150 \mathrm{~m}$ & $\begin{array}{l}125 \mathrm{~m}:+0,87^{\circ} \\
144 \mathrm{~m}:+1,23^{\circ} \mathrm{r}\end{array}$ & 31,54 & $\begin{array}{l}\text { Schlamin mit Schalen: } \\
\text { Balanus porcatus-Ge } \\
\text { meinech. }\end{array}$ & Trawl & $\begin{array}{l}14 \mathrm{Ex}_{1}, 5- \\
23 \mathrm{~mm}\end{array}$ \\
\hline 8 & Safe Bay . & & .15 .7 & $35 \mathrm{~m}$ & - & -. & Fester Schlamm & Iil. Dredge & $\begin{array}{l}3 \text { Ex., } 12- \\
2.2 \mathrm{~mm}\end{array}$ \\
\hline 12 & & & .16 .7 & $118-127 \mathrm{in}$ & $108 \mathrm{~m}:+0,65^{\circ}$ & 34,43 & Loser Schlamm & Tratwl & 1 grosses Ex. \\
\hline 26 & Ymer Bay. & . & .20 .7 & $78-50 \mathrm{~m}$ & $75 \mathrm{~m}:+1,7^{\circ}$ & - & $\begin{array}{l}\text { Fester und zäher } \\
\text { Schlamm }\end{array}$ & Kil. Dredgo & $\begin{array}{l}17 \mathrm{Ex}, 10- \\
22 \mathrm{~mm}(1 \% \\
\text { ovig.) }\end{array}$ \\
\hline 31 & . & $\cdot \cdot$ & .21 .7 & $30 \mathrm{~m}$ & - & - & Foster Schlamm & s & $\underset{\text { min }}{2 \mathrm{Ex}, 19,22}$ \\
\hline 94 & Fjordstamm & $\cdot \cdot$ & . . 21.8 & $147-141 \mathrm{~m}$ & $140 \mathrm{~m}:-0,62^{\circ}$ & 34,491 & $\begin{array}{l}\text { I.oser Schlamm mit kloi- } \\
\text { nen Steinen }\end{array}$ & Trawl & 7 kleine Ex \\
\hline 92 & Nordarm & . $\cdot$ &.$\quad 19.5$ & $85-45 \mathrm{~m}$ & $42 \mathrm{m:}+2,02^{\circ}$ & -1 & $\begin{array}{l}\text { Loser Schlamm mit Kics } \\
\text { und Sand. Am Fnde } \\
\text { der Dredgung Steine } \\
\text { und Iaminarien }\end{array}$ & " & $1 \mathrm{Ex}, 11 \mathrm{~mm}$ \\
\hline 99 & $y \quad$ & . $\cdot$ &. .27 .5 & $197-190 \mathrm{~m}$ & $191 \mathrm{~m}:+0,80^{\circ}$ & 34,721 & Loser Schlamm & » & 1 Ex., $7 \mathrm{~mm}$ \\
\hline 91 & $\begin{array}{l}\text { Nordarm, Lin } \\
\text { die Ekman }\end{array}$ & $\begin{array}{l}\text { ngang } \\
\text { Bay }\end{array}$ & $y^{\prime g} \cdot 19.5$ & $11 \mathrm{~m}$ & {$\left[\right.$ Etwa $\left.3,7^{\circ}\right] \mathrm{vm}$} & - & $\begin{array}{l}\text { Loser Schlamm mit Kies } \\
\text { und Sand; einige Steine } \\
\text { mit Lithothamnion }\end{array}$ & Ki. Dredge & $\begin{array}{l}3 \text { Kleine bis } \\
\text { mittelgrosse } \\
\text { Ex. }\end{array}$ \\
\hline 114 & Ekman Bay & $\cdot \cdot$ & .22 .8 & $27-19 \mathrm{~m}$ & $19 \mathrm{~m}:-0,5^{\circ}$ & $\cdots$ & Zäher, roter Schlamm & n & $\begin{array}{l}+\mathrm{Ex}_{.}, 14- \\
17 \mathrm{~mm}\end{array}$ \\
\hline 119 & $\begin{array}{l}\text { Eingang in di } \\
\text { son Bay. }\end{array}$ & lio Di & ick- & $44-14 \mathrm{~m}$ & - & - & $\begin{array}{l}\text { Strauchfömmiges Litho- } \\
\text { thamnion auf Sichlamm- } \\
\text { boden }\end{array}$ & $\therefore$ & $1 \mathrm{Ex}, 7 \mathrm{~mm}$ \\
\hline 76 & Billen Bay & . &. .13 .8 & $9-10 \mathrm{~m}$ & {$\left[\text { etwa }+5^{\circ}\right]^{X I I I}$} & - & $\begin{array}{l}\text { Kies, Stein und Schalen } \\
\text { mit Lithothamnion (ein } \\
\text { wenig Schlnmm) }\end{array}$ & w & $\left.\right|_{\mathrm{mm}} ^{2 \mathrm{Ex}, 20,26}$ \\
\hline 79 & $x$ & . & . . & $32-40 \mathrm{~m}$ & $\begin{array}{l}{[+1,5 \text { bis }} \\
\left.+2^{\circ}\right] \mathrm{Xv}\end{array}$ & - & $\begin{array}{l}\text { Steino mit strauchför- } \\
\text { migem Lithothamnion }\end{array}$ & ? & 1 grosses Ex. \\
\hline 81 & $\begin{array}{l}\text { Eingang in di } \\
\text { Bay }\end{array}$ & io Bil & Illen 14.8 & $26 \mathrm{~m}$ & $+1,52^{\circ} \times V I$ & 33,77 & $\begin{array}{c}\text { Strauchförmiges Litho- } \\
\text { thamnion, etwas Lies }\end{array}$ & " & $\begin{array}{l}1 \text { kleines, } 1 \\
\text { grosses Ex. }\end{array}$ \\
\hline 83 & Billen Bay & . & .16 .8 & $22 \mathrm{~m}$ & {$\left[\right.$ etwa $\left.+1,8^{\circ}\right]$ xvIt } & - & $\begin{array}{l}\text { Sandgemischter, fester } \\
\text { Schlamm mit Kios und } \\
\text { Stein }\end{array}$ & $n$ & $\underset{\mathrm{mm}}{+\mathrm{Ex}}, 8-14$ \\
\hline 49 & Sassen Bay, & Bank & $k \cdot 31.7$ & $\begin{array}{l}21-19 \text { und } \\
19-28 \mathrm{~m}\end{array}$ & {$\left[+2\right.$ bis $\left.+3^{\circ}\right] \times \mathrm{xx}$} & - & $\begin{array}{l}\text { Stein, Kies und SchaIen } \\
\text { mit Lithothamnion }\end{array}$ & Jrawl & $\begin{array}{l}15 \mathrm{Ex}, 5- \\
15 \mathrm{~mm}\end{array}$ \\
\hline 57 & Sassen Bay & . & . 1.8 & $13 \mathrm{~m}$ & {$\left[+3\right.$ bis $\left.+4^{\circ}\right]$ six } & - & $\begin{array}{l}\text { Schlamm mit Kios, Sand } \\
\text { und Lithothammion- } \\
\text { Bruchstücken }\end{array}$ & Iil. Dredgo & $1 \mathrm{Ex}, 5 \mathrm{~mm}$ \\
\hline 44 & $\begin{array}{l}\text { Eingang in } \\
\text { vent Bay }\end{array}$ & die & Ad- 27.7 & $150-110 \mathrm{~m}$ & $128 \mathrm{~m}:+0,01^{\circ}$ & $3+, 54$ & Loses Schlamm mit Iries! & 'Trawl & $\underset{\mathrm{mm}}{7 \mathrm{Ex}, \mathrm{6}-23}$ \\
\hline 45 & Advent Bay & $\therefore$ & .25 .7 & $70-42 \mathrm{~m}$ & $11 \mathrm{~m}:+1,85^{\circ}$ & 34,18 & $\begin{array}{l}\text { Loser, nber zäher } \\
\text { Schlnmm }\end{array}$ & n & 2 lileino Ex. \\
\hline 73 & $"$ & . &. .11 .8 & $35-30 \mathrm{~m}$ & $\begin{array}{l}{[+2 \text { bis }} \\
\left.+2,7^{\circ}\right] \times \times u\end{array}$ & - & $\begin{array}{l}\text { Balanus porcalus-Go-' } \\
\text { meinsch. Fies und Stein }\end{array}$ & Kil. Uredgo & $\underset{\mathrm{mm}}{3 \mathrm{Ex}, 4-15}$ \\
\hline 95 & Fjordstamm & . &. .21 .8 & $188-181 \mathrm{~m}$ & $\begin{array}{l}{\left[0 \text { bis }+0,5^{\circ}\right]^{\mathrm{mI}}} \\
\left(\mathrm{6i3} \mathrm{m}:-0,11^{\circ}\right)\end{array}$ & & Schlamm mit steinen & Trawl & 2 kleine Fx. \\
\hline
\end{tabular}

K. 8r. Vet. Akad, IIandl. Band 54. X̃:0 7. 


\begin{tabular}{|c|c|c|c|c|c|c|c|c|}
\hline $\begin{array}{l}\text { Nr. } \\
\text { der } \\
\text { Stat. }\end{array}$ & Ort und & Datum & Tiefo & $\begin{array}{l}\text { Wasser- } \\
\text { temperatur }\end{array}$ & $\begin{array}{c}\text { Salz- } \\
\text {.gehalt } \\
\% \text { \%o }\end{array}$ & Bodenbeschaffenheit & Gerät & $\begin{array}{l}\text { Anzahl und } \\
\text { Grösse } \\
\text { (Cänge des } \\
\text { Carapax } \\
\end{array}$ \\
\hline 71 & Coles Buy. & $\ldots .8 .8$ & $\begin{array}{l}14-16 \mathrm{lnd} \\
16-14 \mathrm{~m}\end{array}$ & $\begin{array}{l}{[+2,4 \text { bis }} \\
\left.+3,5^{\circ}\right] \times x \times\end{array}$ & - & $\begin{array}{l}\text { Zuerst Kies, dann } \\
\text { Schlamm und Kies }\end{array}$ & İl. Dredgo & ${ }_{17}^{11 \mathrm{~mm},}$ \\
\hline 126 & Fjordstamm & . . . 30.8 & $47-31 \mathrm{~m}$ & $\begin{array}{l}{[+2 \text { bis }} \\
\left.+3^{\circ}\right]^{x \times v e}\end{array}$ & - & $\begin{array}{l}\text { Balanus porcatus-Go- } \\
\text { meinsch. Etwas Kies: } \\
\text { Schlamm in den Ha- } \\
\text { lmuns-liolonien }\end{array}$ & $=$ & $\begin{array}{l}2 \mathrm{Ex} \text {, his } 20 \\
\mathrm{~mm}\end{array}$ \\
\hline 127 & 。 & $\ldots$ & $25 \mathrm{~m}$ & $\begin{aligned} & 1+3 \text { bis } \\
&\left.+3,5^{5}\right]^{x \times v r}\end{aligned}$ & - & Zither Schlamm & s & $\underset{\text { เกm }}{2 \text { Ex., }} 3-11$ \\
\hline $1: 29$ & " & . . . & $15 \pi$ & - & -. & $\begin{array}{l}\text { Sandgemischter Schlamm } \\
\text { mit Kit's und modern- } \\
\text { den dlgenresten }\end{array}$ & , & $\begin{array}{l}\text { ifx.. 4-1: } \\
\text { num }\end{array}$ \\
\hline $10: 3$ & $\begin{array}{l}\text { Gireen l3ay, ni } \\
\text { Eingang. }\end{array}$ & the heim & $130 \mathrm{~m}$ & $+0,5 \mathrm{~s}^{\circ}$ & - & $\begin{array}{l}\text { Loser Schlnmm. Einige } \\
\text { Steine und Balants } \\
\text { porcatus }\end{array}$ & 'Trawl & $1 \mathrm{kx}, 13 \mathrm{~mm}$ \\
\hline 130 & Gireen Bay & . 308 & $40-45 m$ & - & - & $\begin{array}{l}\text { Schlamm mit Algen-, } \\
\text { resten }\end{array}$ & KI. Dreclgo & $\begin{array}{l}26 \mathrm{Ex}, 5-24 \\
\mathrm{~mm}\end{array}$ \\
\hline 59 & " & $\ldots .3$ & Etwa $40 \mathrm{~m}$ & ... & - & $\begin{array}{l}\text { Sehr loser Schlamm mit } \\
\text { 'Teilen von Land- } \\
\text { pflanzen }\end{array}$ & " & 1 Ex.. $11 \mathrm{~mm}$ \\
\hline bo & , & $\ldots$ & $3: 3 \mathrm{~m}$ & - & - & $\begin{array}{l}\text { Inies, Stein und Schalen' } \\
\text { mit Lithothamnion- } \\
\text { Krusten; zahlreiche Ba- } \\
\text { lanusporcatus-Kolonien }\end{array}$ & \& & $\begin{array}{l}3 \text { kleine, } 3 \\
\text { grössere bx. }\end{array}$ \\
\hline 61 & , & $\ldots .4$ & $40-3.5 \mathrm{~m}$ & ... & - & $\begin{array}{l}\text { Kies unl Stein. Balantrs } \\
\text { porcalus Gemeinsch. }\end{array}$ & $\begin{array}{l}\text { Hi. Hummer- } \\
\text { körbchen }\end{array}$ & $\begin{array}{l}\text { It lix..9-11 } \\
(3 \text { Ex. } 15 \\
-17(3 \mathrm{Ex}) \\
20-20 \\
\text { Ex.) mm (I } \\
\text { Q ovig.) }\end{array}$ \\
\hline 03 & $\begin{array}{l}\text { Green Bay. } \\
50 \mathrm{~m} \text { vom } \\
\text { les Alitebrr } \\
\text { schers.. }\end{array}$ & $\begin{array}{l}\text { Etwa } \\
\text { Rande } \\
\text { ra-Glet- } \\
. \quad 58\end{array}$ & $16 \mathrm{~m}$ & - & - & Ioser Schlamm & Iil. Dredge & $1 \mathrm{Ex}, 20 \mathrm{~mm}$ \\
\hline 63 & Green Bay & ...5.s & $15 \mathrm{~m}$ & - & - & " & » & $1 \mathrm{Fix}, 7 \mathrm{~mm}$ \\
\hline
\end{tabular}

[Ausser im Eisfjord fanden wir Eupagurus pubescens im IIo r n s u n d (Gices' Bay, 10.7; 25 m, Sehlamm mit etwas Kies, 1 F.x.; $10-35 \mathrm{~m}$, Schlamm und Kies, 5 Ex.).]

Frithorotynde im Eis f jord:

Scluwed. Exped. 1858-1873: Safo Bay, 32, 36 his 72,36 his 90, 54 bis 90 m; Skans Bay (Billen Bay), 13 bis $18,27 \mathrm{~m}$; Sassen Bay, $36 \mathrm{~m}$; Alrent Bay, 5,5 his 9, 9 bis 18, 22 bis $36,45,36$ his 54,72 bis $108 \mathrm{~m}$; Coles Bay, 18 bis $46 \mathrm{~m}$; Green Bay, 9 bis 36, 54, 72 bis $90 \mathrm{~m}$ (Riksmuseum, Stockholm, nach A. MoldNDER).

Miers 1877: Green Bay. Schwed. Exped. 1890: Green 13ay, 35 bis 72 m (KLinchowström 1892). Schwed. Exped. 1898: Safe 13ay, 50 his $90 \mathrm{~m}$, Schlamm; 1899: Green Bay, $110 \mathrm{~m}$; 1900: Coles Bay, $50 \mathrm{~m}$, steinig (Omly 1901). Russ. Exped. 1899, 1900: Adrent Bay, 9 m, Steine; 0 his 16 m, Grus (Bmula 1907). Belgica-Expod. 1905: Green Bay (GriEg 1909).

Wir fanden Enpagurus pubescens an 32 Stationen, in zusammen 160 Individuen; er ist also ungefähr ebenso häufig wie Spirontocaris spinus und turgida.

Auf losem, ungemischtem Schlammboden lebt diese Art nur ausnahmsweise und vereinzelt (St. 12, 99, 63, 65); häufiger ist sie auf festerem Schlammboden (St. 8, 26, 31, 114, 127) und besonders an mit Kies, Steinen oder Schalen gemischtem Schlamm (St. 13, 94, 92, 91, 83, 57, 4t, 95, 129, 103). Auf Steingrund mit Laminarien fanden wir sie nicht, zweifellos weil die Laminarien hauptsächlich auf die Uferzone beschränkt sind. Auf Lithothamniongrund fanden wir sie nicht selten, doch nur einmal (St. 49) in grösserer 
Individuenzahl. Sehr allgemein ist diese Art an Stellen mit mehr oder weniger reichlichem Balanus porcatus, und hier lebt sie oft in verhältnismässig grosser Individuenzahl.

In vertikale Richtung erstreckt sich die Verbreitung im Eisford von $10 \mathrm{~m}$ bis nahezu $200 \mathrm{~m}$. Schon von der oberen Verbreitungsgrenze oder wenigstens von etwa $15 \mathrm{~m}$ an ist die Art, wie die nachstehende Übersicht zeigt, häufig wenigstens bis in $150 \mathrm{~m}$ 'Tiefe, Das Fehlen in der unteren Hälfte des Fjords muss zweifellos der ungünstigen Bodenbeschaffenheit zugeschrieben werden.

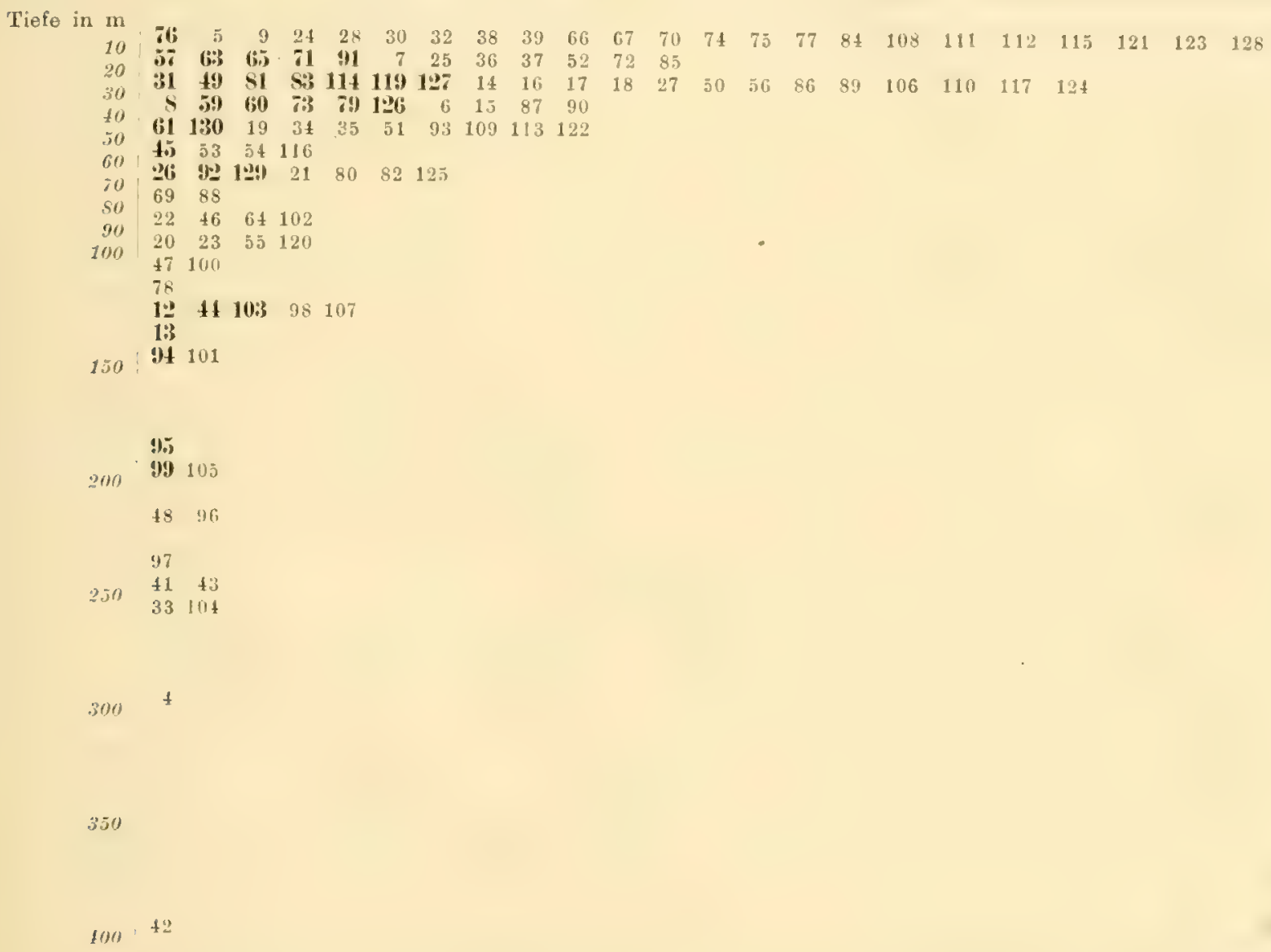

Wie das Schema zeigt, fanden wir die Art nicht zwischen etwa 65 und etwa $120 \mathrm{~m}$, obgleich sie in etwas grösserer Tiefe wieder wenigstens ebenso allgemein wie oberhalb von $70 \mathrm{~m}$ ist. Obgleich die Anzahl der zwischen 70 und $120 \mathrm{~m}$ untersuchten Stationen nicht sehr gross ist, dürfte diese eigentumliche Luicke in der vertikalen Verbreitung nicht scheinbar sein. Sie fällt fast genau mit der Kaltwasserschicht zusammen, welche nach unsern Untersuchungen zwischen dem warmen 'Tiefen- und dem ebenfalls warmen Oberflächenwasser eingeschaltet ist. Darf man num auf einen ursächlichen Zusammenhang zwischen diesen zwei Erscheinungen schliessen, dem Vorkommen einer intermediären Kaltwasserschicht und dem Fehlen von Eupagurus pubescens zwischen 70 und 120 m? Wenn keine andere Griunde vorlägen, wiirde dieser Schluss zweifellos iibereilt sein, man liommt aber' auf anderem Wege zu Ergebnissen, welche diese Annahme bestärken. 
Die W assertemperat $\mathrm{rr}_{\mathrm{r}}$ der Fundorte schwankte zwischen etwa $+5^{3}$ und $-0,62^{\circ}$. E. pubescens ist, wie die machstehende Ubersicht zeigt, seltener im Wasser ron negativer 'Temperatur als irgend eine andere Decapodenart; im kältesten Wasser des Fjords wurde er gar nicht gefunden. Unter den 22 Stationen mit negativer 'T'emperatur, wo das 'Tier fehlte, gibt es jedoch mehrere, wo man sein Vorkommen kaum erwarten könnte (10 oder 11 mit reinem losem Schlammgrund, 4 nahe am Gletscherrand gelegen); auch das Fehlen an den ibrigen kann wohl an sich nicht als ein genuigender Beweis für die Annahme erklärt werden, dass die Art Wasser von negativer 'Temperatur meidet. Die allgemeine Verbreitung liefert jedoch die kräftigsten Stützen für diese Auffassung; die Art lebt zwar in Nord- und Ostspitzbergen (wenigstens im Storfjord), fehlt aber so gut wie vollständig in allen ausgesprochen hocharktischen Gegenden: Karisches Heer und Sibirisches Eismeer mit Ausnahme des östlichsten 'T'eils, Nordostgrönland, Nordwestgrönland und benachbarte 'Teile des nordamerikanischen Archipels.

Unter diesen Umständen hat man gute Grinde zu vermuten, dass die Verbreitung im Eisfjord durch die T'emperaturverhältnisse beeinträchtigt wird und dass auch die eigentiimliche, sonst schwer verständiche Lücke in der vertikalen Verbreitung in dieser Weise zu erklären ist. Doch kann das T'ier zweifellos wenigstens zeitweise in Wasser ron negativer Temperatur leben, wie z. B. die Funde in Nordostspitzbergen zeigen. Die Totalität der Liicke beruht daher wahrscheinlich auf andern Umständen; bei zahlreicheren Dredgungen hätten wir wohl das 'Tier auch zwischen 70 und $120 \mathrm{~m}$ gefangen, obgleich seltener als sonst.

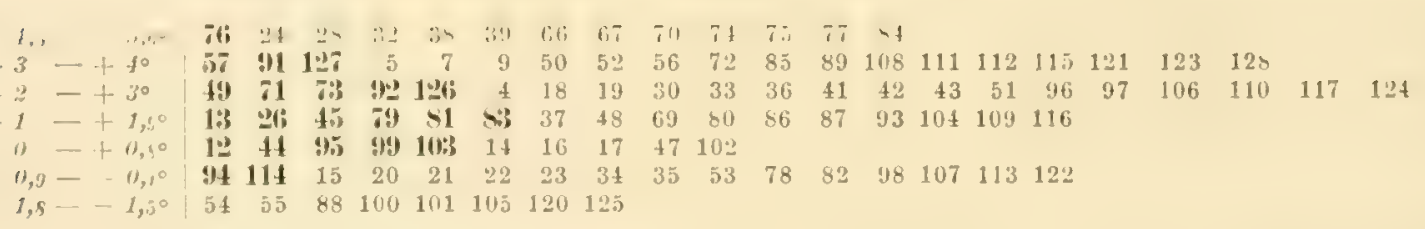

Im warmen Oberflichenwasser ist $E$. pubescens sehr selten; nur ausnahmsweise wurde er in Wasser von mehr als etwa + $3^{\circ}$ Temperatur erbentet. Eine Einwirkung der Wassertemperatur dürfte jedoch ausgeschlossen sein; das Tier hat in allen arktischen Gegenden ungefähr dieselbe obere Verbreitungsgrenze und erträgt in der borealen liegion viel wärmeres Wasser.

Fortpflanzung, Entwicklung. In der ganzen Sammlung finden sich nur 3 eiertragende Weibchen, am 10.7 (Hornsund), 20.7 und 4.8 gefangen; ihre Carapaxlänge beträgt $12,5,13,5$ und $20 \mathrm{~mm}$. Die Eier befinden sich in unentwickeltem (oder jedenfalls sehr wenig entwickeltem) Zustande; dasselbe gilt ron einem von der Kolthoff-Expedition am 25. Juni 1900 in der Green Bay gefangenen Weibchen. Aus diesem spärlichen Material kamm man natürlich keine Schlïsse auf die Entwicklungszeit ziehen; höchstens kann man sagen, dass die Entwicklung wahrscheinlich entweder teilweise oder ausschliesslich in den Herbst oder Winter fällt. 


\section{Allgemeine Verbreitung.}

(1ing. 9.)

śpitzhergengebiet:

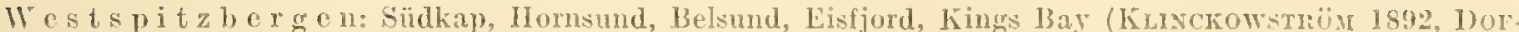
LEIX 1900, OHLIN 1901, BrRula 1907, Dovs 1915 und oben; ferner S.ses 1886: Spitzbergen). No rd West.

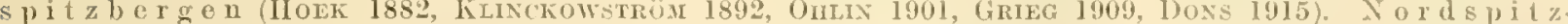
b erg e n: Liefde Bay, Treurenburg 13ay, Nordeingang in die Hinlopen-Strasse, $N$. und No. vom Jordostland (Dorlein 1900, OmLin 1901, Grieg 1909). O s t s p it z bergen: Edges Land, Hopen Eiland, Storfjord und S. davon (Dorlein 1900, Oilm 1901, Brinula 1907, Doxs 1915). B o e y e n E il a n d; zwischen dem Südkap und

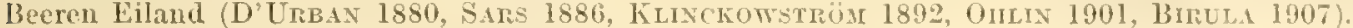

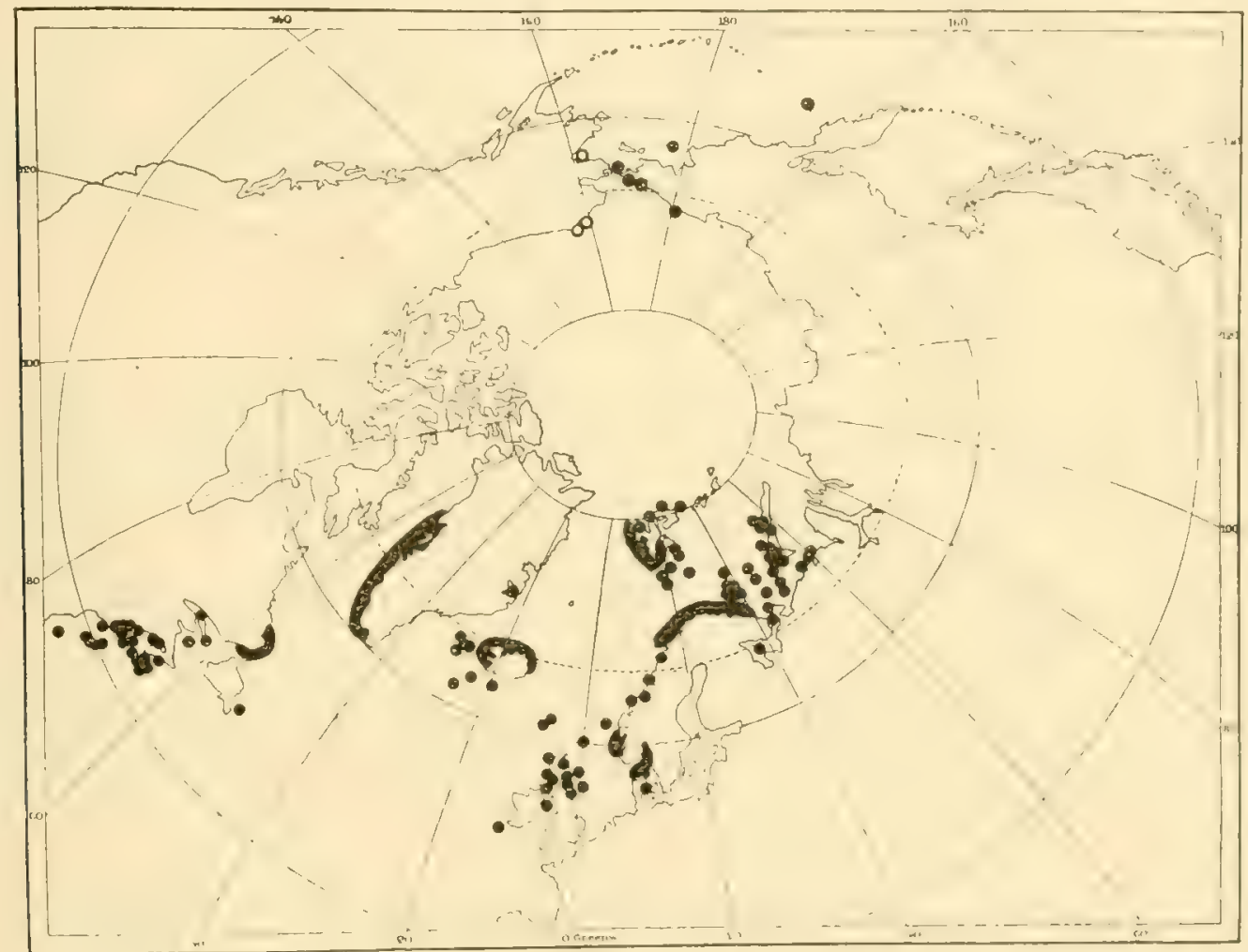

Fig. 9. Eupagurus pubescens; $O$ die nördlichsten Fundorte für E. trigonocheirus.

Übrigearktise he und boreoarktische liegion:

Jugor schar, Matotschkin sehar, Westküste von Novaja Semlja und südlicher 'Teil des Barentsmeeres

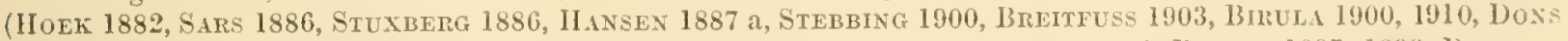

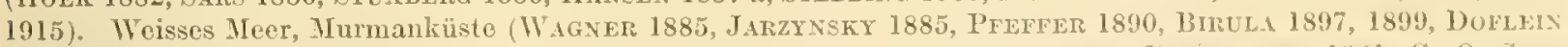
1900, DERJUGIN 1906, 1912, AWERINZEW 1909). Ostfinnmarken (II. SARS 1858, DANIELsSHN 1861, G. O. S.IRE 1883, Norman 1902, Nordgated 1905, Doxs 1915). Kalte Fjorde im nordwestlichen Norwegen (s. unten).

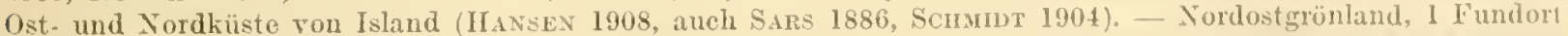
im südlichsten Teil (HAXsen 1908). Westgrönland, nördlich bis $72^{\circ} 37^{\prime} \mathrm{n}$. Br. (STLPHixsis 1913,1916 und frühcro Autoren). - Nordostküste von Labrador und Newfoundland, Golf von St. Lawrence, Ostküste von Nordamerika

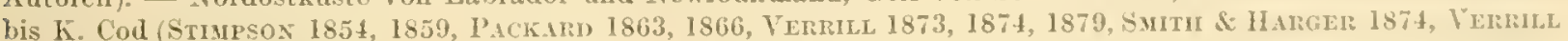

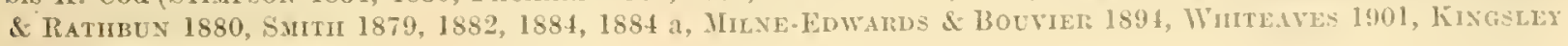
1901, Schmit 1904, Rathide 1905). 
P'a ifisclics (ie l, iet:

Im Jeringsmecr und an den Küsten südliel davon leben einige mit L. pubesecns äusserst nahe verwandto Eupagurus-Formen: F. trigonocheine Stmps. (E. cupillatus BexEnct mit dem vorigen identisch, s. 13atas 1913),

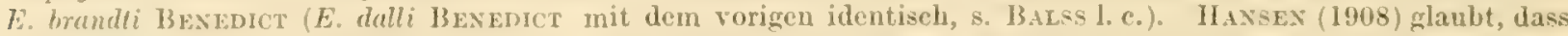
wenigstens dio erstgenannte Form mit E. pubescens zu vereinigen ist. Nach B.ALs (1913) dagegen handelt es sich un eine von der atlantischen Form getrennte species; nder echte F. pubescens kommt im Pazifik nicht vor».

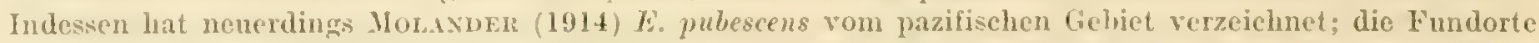
(Vega-Expedition) liegen teils in der Beringsstrasse und an der Jordküste von sibirien an deren Eingang, teils im nordwestlichon Teil des beringsineers und an der l3ering-Insel. Schon STuxibra (1882) erwälnt den Fundort an Eingang der Beringsstrasse, ferner zwei Fundorte weiter westlich an der sibirischen Küste. Ein Vergloich mit E. trigonocheine wird nicht gegehen, Dr. MoLAvink hat mir aber freundlichst mitgeteilt, dass die Exemplare von der sibirischen Küsto völlig normal sind; anch die Exemplare vom Beringsineer können nicht von $E$. pubescens getrennt werden, obgleich einige ein wenig an L: trigonocheims erinnern.

E. pubescens ist also unwiderleglich im nördlichsten Teil des pazifischen Gebiets oder jedenfalls im Eismeer nördlich vom Beringsmeer rerbreitet. Über die Beziehungen zwischen ihm und E. trigonocheirus kann ich mich nicht äussern; anf der Karte Fig. 9 habe ich diese Form nicht berücksichtigt, obgleich mehrere Fundorto wahrscheinlich nit demselben Recht hätten aufgenommes werden künnen, wie die südlichen Fundorte der Vega.Expedition. Die nördlichsten Fundorte fïr P’. trigonocheirus (Munocn 1885) habe ich auf der Karte durelı Ringe bezeichnet; wenn die sihirischen Exemplare zum typisehen E. pubescens gehören, kann man kaum erwarten, dass an der Fortkitiste Alaskas nur cine andere Form rorkommt.

$$
13 \text { orealo } \operatorname{Region:}
$$

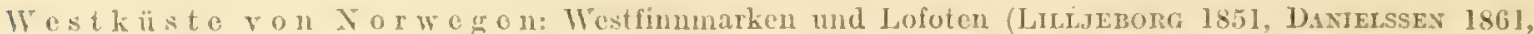

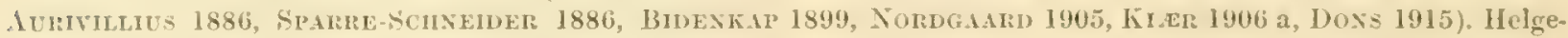

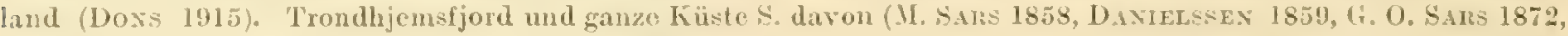

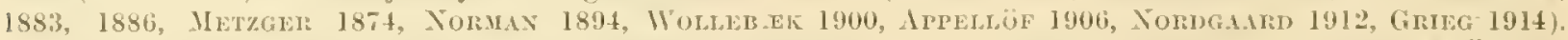

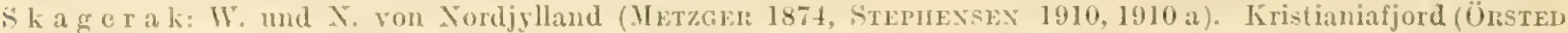

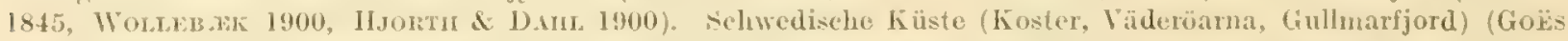

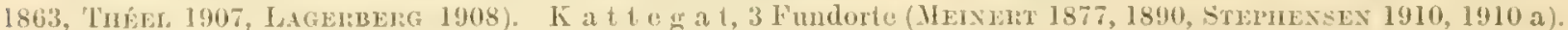

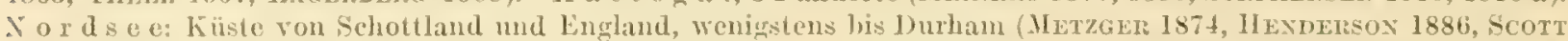

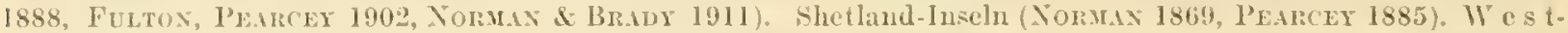

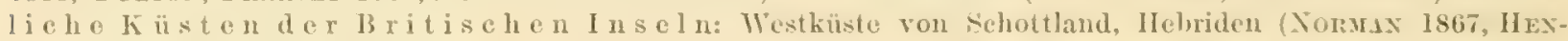

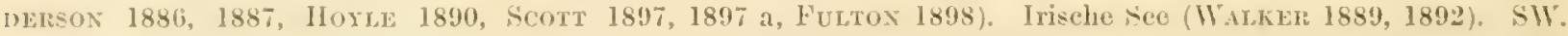

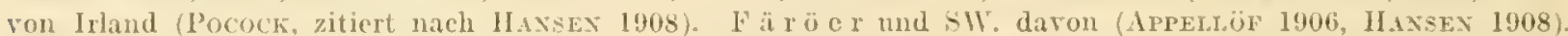

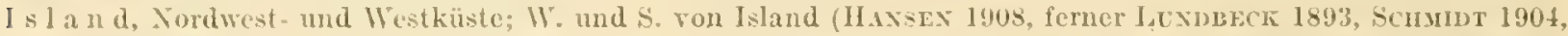

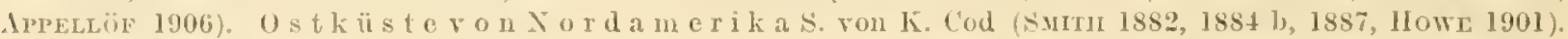

Bathymetrische Verbreitung. In der arktischen Region ist Eupagurus pubescens bisweilen in noch geringerer I'iefe als im Eisfjord gefunden worden (PACKARD 1866: "from low-water mark»; SHTH 1884: 5,5 $\mathrm{m}, 2$ bis 5,5 $\mathrm{m}$; STUXBerg 1886: 5,5 bis $9 \mathrm{~m}$; Ortuaxy 1901: 0 bis $1 \mathrm{~m}$; vgl. auch Doxs 1915), doch sind solche Funde selten, und er wird ïberall erst bei $10 \mathrm{~m}$ etwas häufiger, wirklich allgemein wahrscheinlich erst bei etwa $20 \mathrm{~m}$. Dasselbe gilt für die boreale Region, wo das Tier nie im oberen Teil der Litoralregion vorzukommen scheint (vgl. Z. B. AppeliöF). Die tiefste Fundstelle liegt nach HaNSEx (1908) bei $1150 \mathrm{~m}$ (Ostkiiste yon Nordamerika). Die untere Grenze für das häufige Vorkommen liegt jedoch bedentend höher. Nach mehreren Beobachtungen ist die Art stellenweise allgemein - ob ebenso allgemein wie oberhalb von $200 \mathrm{~m}$ muss dahingestellt werden - bis in etwas mehr als $500 \mathrm{~m}$ 'Tiefe (s. besonders HANSEx 1908, fermer Sumti 188+b, 1887, SARs 1886, Dofleis 1900, Stephensex 1912 a). Von noch grösserer Tiefe liegen einige Funde vor (HANSEN 1908: 650, 810, 873, 875, 765-900 m; APPELLör 1906: $840,950 \mathrm{~m}$; vgl, auch oben). 


\section{Hyas araneus (L.).}

Unsere Exemplare dieser Art - und dasselbe gilt von den von der Kolthoff-Expedition $1900 \mathrm{im}$ Eisfjord gesammelten — zeigen das für die arktische Region charakteristische Aussehen. Sie gehören also zu der von Birula $(1897,1907,1910)$ als var. hoeki bezeichneten Form; dass die Untersehiede konstant und erblich fixiert sind, ist. natiirlich keineswegs erwiesen.

\section{Verloreitume in Eisfjord.}

Verzeiehnis der lindorte:

\begin{tabular}{|c|c|c|c|c|c|c|c|}
\hline $\begin{array}{l}\text { Nr. } \\
\text { der } \\
\text { Stat. }\end{array}$ & Ort und Datum & Tielo & $\begin{array}{l}\text { Wasser- } \\
\text { temperatur }\end{array}$ & $\begin{array}{c}\text { Salz- } \\
\text { gehalt } \\
\% 00\end{array}$ & Bodenbeschaffenheit & Gerät & $\begin{array}{l}\text { Anzahl und } \\
\text { Grösse } \\
\text { (Liinge des } \\
\text { Carapax) } \\
\end{array}$ \\
\hline 13 & $\begin{array}{c}\text { Eingang in dio Safo } \\
\text { Bay } \ldots \ldots .7\end{array}$ & $125-150 \mathrm{~m}$ & $144 \mathrm{~m}:+1,23^{\circ} \mathrm{n}$ & 34,54 & $\begin{array}{l}\text { Schlamm mit Schalen; } \\
\text { Balanus porcahrs-Ge. } \\
\text { meinsch. }\end{array}$ & 'Trawl & I Ex., $8 \mathrm{~mm}$ \\
\hline 120 & Dickson Bay . . . . 27.8 & . $98 \mathrm{~m}$ & $93 \mathrm{~m}:-1,03^{c}$ & 34.27 & Loser Srhlamm & " & $1 \mathrm{Ex}$ \\
\hline 47 & $\begin{array}{l}\text { Eingang in dic Sassen } \\
\text { Bay }\end{array}$ & $97-120 \mathrm{~m}$ & $\mathrm{~s}^{2} \mathrm{~m}:+1,71^{\circ}$ & 34,18 & $D \quad D$ & Ottertrnwl & 1 f, $34 \mathrm{~mm}$ \\
\hline 75 & $\begin{array}{l}\text { Advent Bay, auf einer } \\
\text { Bank }\end{array}$ & $6 \mathrm{~m}$ & {$\left[\text { etwn }+4,5^{\circ}\right]^{x \times m}$} & - & Stein mit Laminarien & Kil. Dredge & $\begin{array}{l}10 \%, \text { etwa } \\
48 \mathrm{~mm}\end{array}$ \\
\hline 95 & Fjordstamm . . 21.8 & $188-181$ in & $\begin{array}{l}{\left[0 \text { bis }+0,5^{\circ}\right]^{\mathrm{vm}}} \\
\left.16 \mathrm{in}:-0,11^{\circ}\right)\end{array}$ & - & Schlamm mit Stcinen & Trawl & $107,88 \mathrm{~mm}$ \\
\hline 129 &. $.30 \mathrm{~s}$ & $65 \mathrm{~m}$ & - & - & $\begin{array}{l}\text { Sandgemischter Schlamm } \\
\text { mit Kies und modern- } \\
\text { den Algenresten }\end{array}$ & Ki. Dredge & I $03,50 \mathrm{~mm}$ \\
\hline $61 \mathrm{a}$ & . . 4.8 & $35 \mathrm{~m}$ & - & - & $\begin{array}{l}\text { Kies und Stein. Balanus } \\
\text { porcatus Gemeinsch. }\end{array}$ & $\begin{array}{l}\text { Hummer- } \\
\text { körbchen }\end{array}$ & I $\mathrm{o}^{2}, 58 \mathrm{~mm}$ \\
\hline 60 & $\cdots 3.8$ & $33 \mathrm{~m}$ & - & - & $\begin{array}{l}\text { KKies, Stein und Schalen } \\
\text { mit Lithothamnion- } \\
\text { Krusten; zahlreiche Ba- } \\
\text { lanus porcatus-Kolonien }\end{array}$ & Kil. Dredgo & $107,13 \mathrm{~mm}$ \\
\hline
\end{tabular}

[Ausser im Eisford fanden wir ein kleines Exemplar im Hornsund (Goës Bas, 10.7, 10 his 35 m, Schlamm und Kies)].

$$
\text { Frïlore Funde in Eisford: }
$$

Schwed. Exped. 1864: Safo Bay (Riksmuseum, Stockholm). Miers 1877: Green Bay, 55 m. Ruks. Experl. 1899, 1900: Adrent Bay, $9 \mathrm{~m}$, Steine; Fjordstamm vor der Advent Bay, $243 \mathrm{~m}$, Temp. —0, $8^{\circ}$, Schlanm und Grus (Brrula 1907). Zweifellos auch schwed. Exped. 1890: Green Bay, 35 his 72 m (Kuxckowström 1892 [\#11yas 》). OnLms nimmt keinen Fund im Eisfjord auf, in den sammlungen der Kolthoff-kxpedition 1900 finde ich aler 4 mittelgrosso Exemplare, in der Green Bay in 10 bis $80 \mathrm{~m}$ Tiefe auf steinigem Boden gefangen.

Wir fanden Hyas araneus an 8 Stellen, an jeder in einem Lixemplar. Lir ist also der unvergleichlich seltenste der Eisfjorddecapoden, obgleich der Unterschied wohl nicht so gross ist, wie aus einem blossen Vergleich der Zahlen hervorzugehen seheint; die geringere Ausbente beruht natürlich teilweise auf der Lebensweise der Art, die nicht, wie die Macruren, in grossen Scharen lebt. 
Die Fundorte zeigen die verschiedenste $B$ o d e $n \mathrm{~b}$ e s e h a f e $\mathrm{n} h \mathrm{e} i \mathrm{t}$, und die Art fehlte auch auf dem ungemischten Schlammgrund nicht, obgleich sie zweifellos dort selten ist; verhältnismässig am häufigsten scheint sie in den Balanus porcalus-Gemeinschaften zu sein.

Die vertika le Verbreit ung erstreckt sich von 6 bis etwa $185 \mathrm{~m}$. Der oben erwähnte Fund einer russischen Expedition in $243 \mathrm{~m}$ Tiefe zeigt, dass die von uns gefundene untere Grenze überschritten werden kann; noch tiefer dïrfte das Tier jedoch kaum hinabsteigen, da die grösste bisher beobachtete Tiefe $310 \mathrm{~m}$ beträgt. Oberhalh

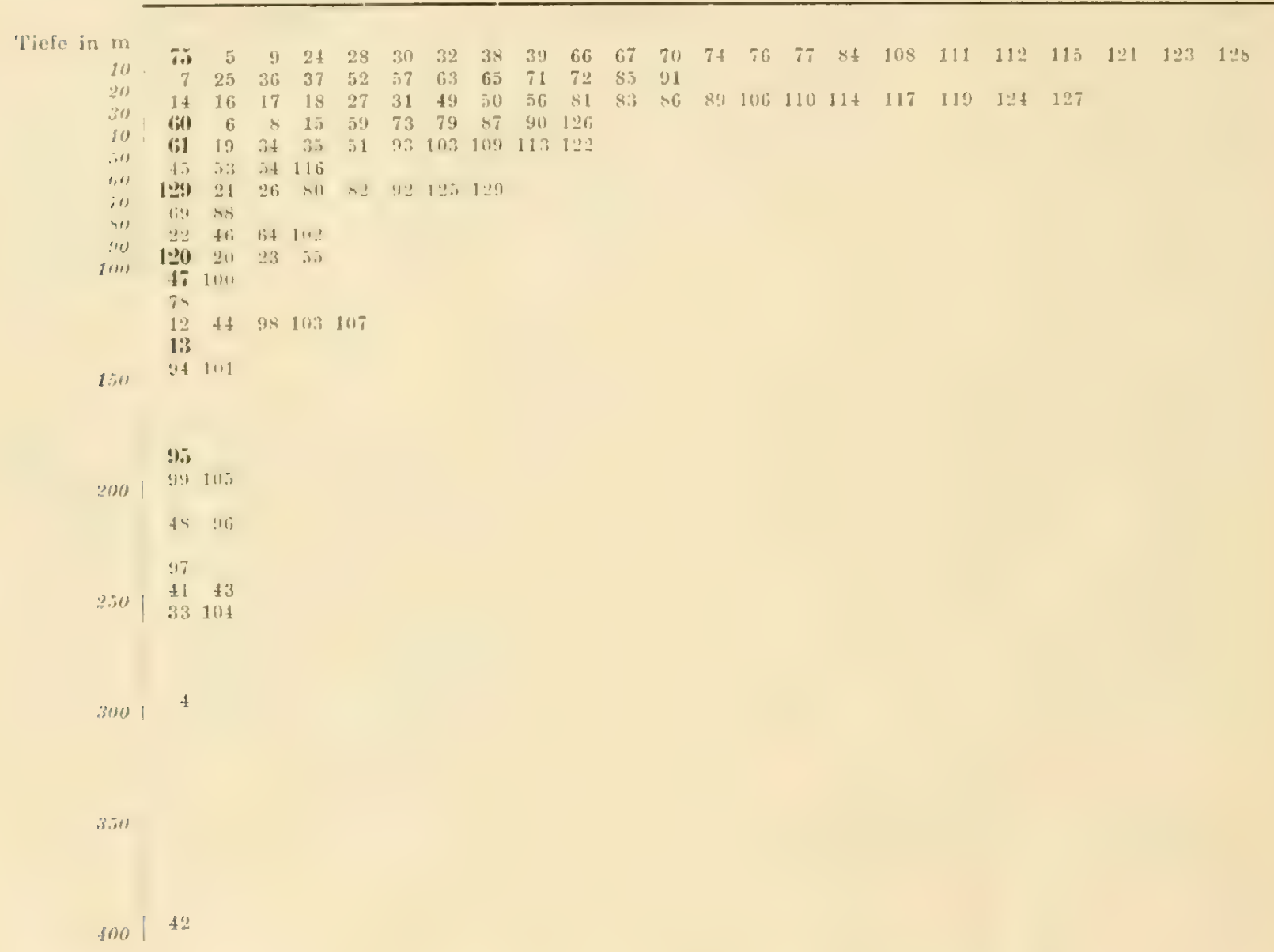

von $6 \mathrm{~m}$ dürfte es nicht oder höchstens nur ganz zufällig auftreten,. Bis zu 30 oder 20 in hinab muss die Art, wie die vorstehende Übersicht veranschaulicht, äusserst selten sein. Mit zunehmender Tiefe, wenigstens bis etwa $125 \mathrm{~m}$, wird sie eher häufiger als seltener.

In bezug auf die $\mathrm{W}$ as sertemperatur liegen nur 5 Beobachtungen vor. In Wasser von negativer 'Temperatur fanden wir die Art einmal; bei der geringen Anzahl der Beobachtungen beweist dies gar nichts. Die allgemeine Verbreitung zeigt dagegen iiberzeugend, dass sie in der Regel negative 'lemperaturen nicht erträgt; sie ist selten in Ostspitzbergen (s. unten) und fehlt bei Nordost- und Nordwestgrönland usw. 


\section{Illgemeine Terbreitung.}

(Fig. 10.

Spitzborgengebiot:

Westspitz bergen: Hornsund, Belsund, Eisfjord (Onim 1901, Biruta 1907, Dons 1913, 1915 und oben; ferner Sars 1886: Spitzbergen). Nordwestspitzborgen (Doflein 1900, Hartlaub 1900, Grieg 1909). Ostspitzbergen: Great Insel (Nordostland), Storfjord (südwestlichster Teil) und Deevio Bar sowic S. und SW. davon, Hopen Eiland (Doflein 1900, Haitraub 1900, Stebbing 1900, Ohlin 1901, Birura 1907 , Doxs 1913, 1915). B e e r en Eiland (SARs 1886, Doflein 1900, OIILIN 1901, Birula 1907).

IIyas araneus ist also an der Westküsto ziemlich allgemein; an der Nordküste ist er gar nicht, in Ostspitz. bergen einmal im nördlichen, sehr kalten Gobiet, sonst nur im Süden unweit der wärmeren Gegenden gefunden worden. Im übrigen Ostspitzbergen muss er sehr selten sein, da er weder von der schwedischen Expedition 1898,

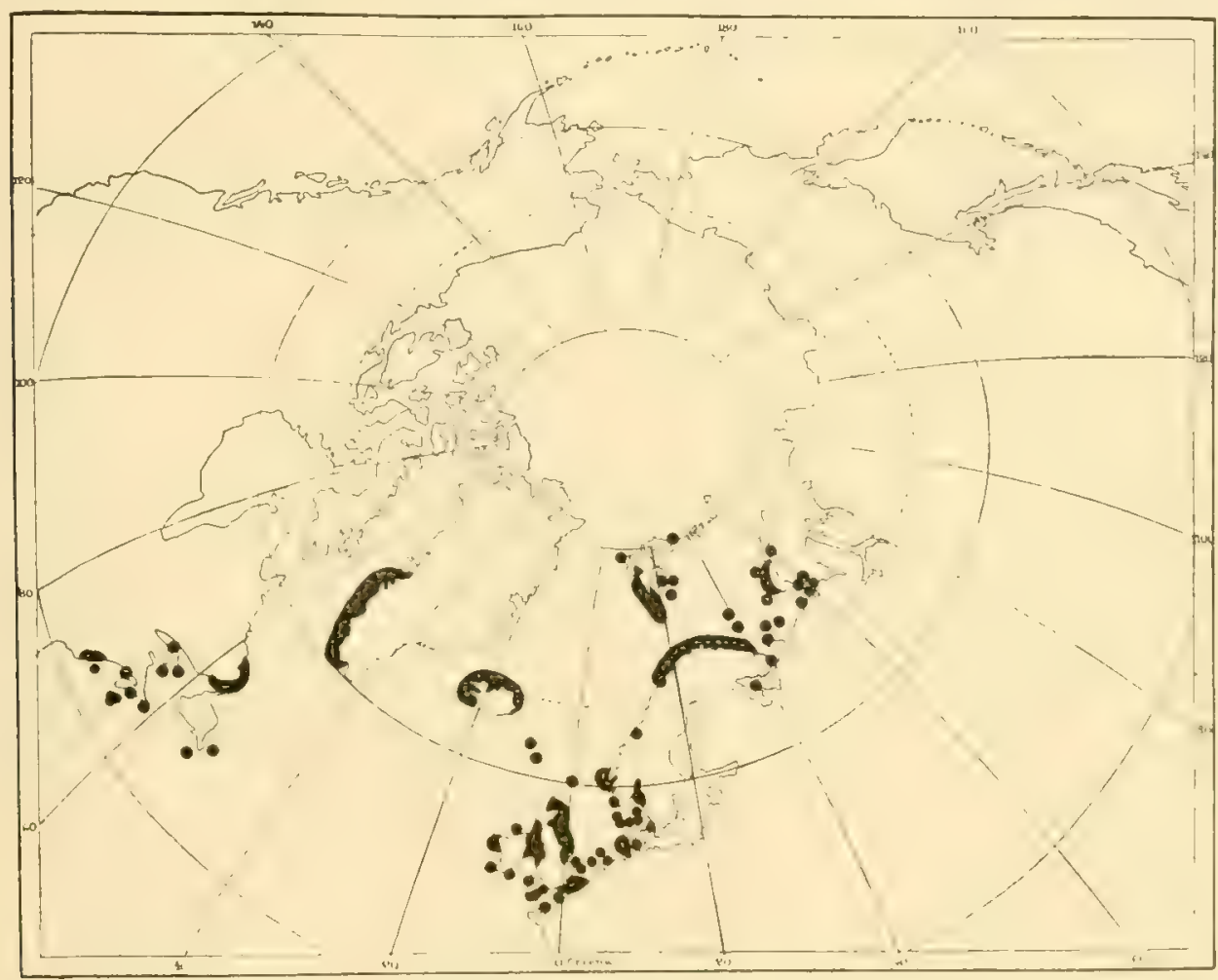

Fig. 10. Hyas arancus.

noch von den russischen Expeditionen dort erbeutet wurde. Für DOFLEIN's Ansicht, dass dio Art cin ausgosprochener Kaltwasserbewohner sei, in Ostspitzbergen in oberflächlichen Schichtan, im Westen hauptsïchlich im kalten Wasser lebend, lar auch in jener Zeit nicht der Sehatten eines Beweises vor.

$$
\text { Übrigo arktischo und boreoarktische liogion: }
$$

Karisches Meer, westlichster und südlichster Teil (STUXBERG 1882?, 1886? [II. coarclalus], IIANSEN 1887 a). Karische Pforte, Westküste von Noraja Semlja, südicher Teil der Barentsmeeres (D'Uriß. 1880, HoEk 1882,

1 STUXerig crwihnt 1882 Myas araneus aus dem Karischen Meer (Ostküsto ron Novaja Semlja) und aus dom östlichsten Teil der sibirischen Nordküste (zwischen dem Karischen Meer und $177^{\circ} 4 \mathbf{l}^{\prime} \ddot{0}$. Is. wurdo den Angaben Stuxberg's nach zu urteilen keine Hyas-Art ron der sehwedisehen Expedition gefunden). In der späteren Arbeit

K. Sv. Vet. Akad. Handl, Bani รัt. N:n 7. 


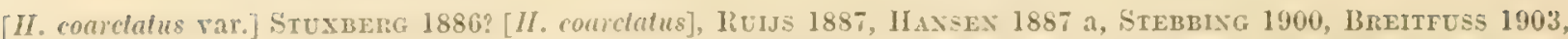
Brrula 1900, 1910, Stappers 1911, Doxs 1913, 1915). Weisses Meer, Murmanküste (Jarzyasky 1885, PrefFer 1890, STIEREx 1895, Brruta 1897, 1899, Dofleis 1900, Derudars 1906, 1912, AWerixzew 1909). Ostfinnmarken (M. SARS 1858, DANiftasex 1861, NomMAN 1902). Nordwestnorwegen (s. unten). - Ost-und Nordküste von Island (HAXSEx 1908, SCHMid 1901). - Westküsto ron Grönland, mördlieh bis (iodhara (STEPHExSEx 1913, 1916 und frülıce Autoren). - Nordostküste yon Isabrador, Golf von St. Lawrence, Newfoundland und Ostküste von Nordamerika bis $K$. Cod (Stmipsox 1859-60, Packard 1863, 1866, Verrill 1874, Suitu \& Harger 1874, Surtir

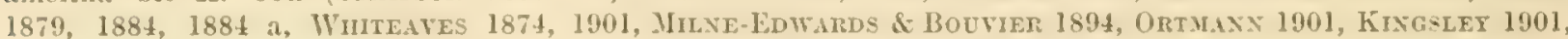
Scmuti 1904, Ritubux 1905). - [Ochotskisches Meer? (Brasdi 1851)'.]

$$
\text { Boreale legion: }
$$

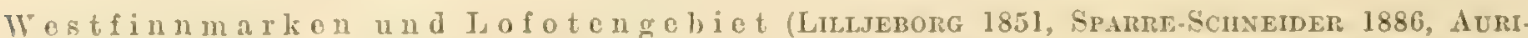

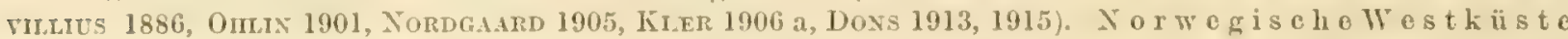
siddlich daron (M. Sars 1858, Daxielssex 1859, G. O. Sars 1872, MetzGer 1874, Storir 1880, dppellüF 1906, Nordgatrd 1912, Girieg 1914). S kagora k: Kiristianiafjord, Brevilisfjord (Wolleb.eK 1900); schwedische

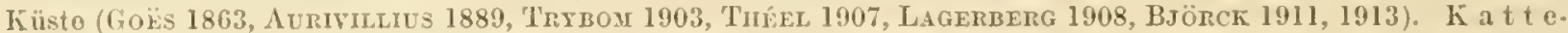

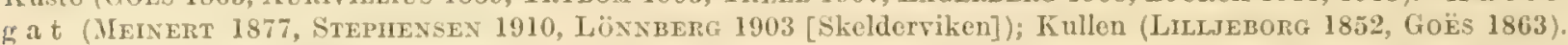
Ó ros und (Örsted 1844, Metrert 1877, 1890, Stephiensex 1910, 1910 a, Lagerberg 1908, BJörck 1913), K i o lo r b u c ht (JEINERT 1890).

Nords o e: Shetlandinseln (Normax 1869), Küsto von Jylland, Helgoland (MöBius 1873, 1893, Metzger: 1874, Meinert 1877, 1890, Dalla Torre 1889, Cunvingiram 1895, Stepiifnsen 1910, 1910 a). Ostküste ron Schottland und England (Leicir 1815, Dalyeli 1851, Belz 1853, Metzger 1874, M'Ixtosir 1875, Lescie \& Herd MaN 1881, Scott 1888, Fultox 1890-98, Peatcer 1902, Petcir 1903, Her 1903, Redeke 1906, Mar. Biol. Assoc. 1909, Norman \& Bradr 1911). Südliche Nordseo vor der Küste ron Holland (Garstang 1905, Redeke 1906); Ooster-Scheldo (HOEK 1883). Küsto von Belgien (V. BENEDEx 1861). En g l is c hor K a $n$ a l: Südküsto ron Fngland (Belu 1853, Heape 1888, Garstaxg 1892, Mar. Biol. Assoc. 1909, Nommax \& Scott 1906); Kanalinseln (Koemler 1886, Normas 1907). [Ferner Milve-Edrands 1834-40: "Côtes d'Angleterro et de la Franco».] Wostküste you Seluottland, I r ische See (HASSAL 1812, Trompson 1842, 1844, 1856, BeLL 1853,

ron 1886 wird nur der Fund im Karischen Meer erwähnt, ausserdem aber mehrere Funde an der Westküste von Novaja Semlja hinzugefügt; in dieser Arbeit wird ein anderer Speciesname, coarctatus LEAcr, gebraucht.

Dio Novaja-Semlja-Form dürfte num, wio Brrura (1907) bemerkt, eher zu II. araneus gehören, denn ron allen andern Expeditionen ist in diesem Gebiet nur eine Form von dieser Art gefunden worden; die von HoEk (1882) als "II. coarctatus var." bezeichnete Form gehört jedenfalls sicher hieher (vgl. BIRULA, 1. c. [S. 8] und 1909).

Dio am östlichsten Teil der sibirischen Küsto gefundenen, bloss in der ersten Arbeit erwähnten Tiere werden ron BrEura (1907) als wahrscheinlich zu coarctatus gehörig bezeichnet, weil im nordpazifischen Gebiet nur eine Form ron dieser Art rorkommt. DoNs (1913) dagegen findet neuerdings, dass sio Richtigkeit der Bestimmung STUXnEIEG's nicht angezweifolt worden könne, weil man sonst voraussetzen müsse, dass dieser Forscher die Merkmale von araneus für coarctatus habe gelten lassen und umgekehrt. Diese Auffassung beruht auf einem Missverständnis; STUXBEIE hat in seinem Material nicht zwei Arten untersehieden, sondern einfach für eine seiner $\Lambda$ uffassung nach einheitliche Art zuerst den einen, später den andern Namen gebraucht (die Lixemplare vom líarischen Meer 1882 araneus, 1886 coarctatus genannt.) Nach einer persönliehen Mitteilung von Dr. A. MoLAxDER, der mit einer Bearbeitung der arktischen und mordischen Decapoden des Zoologischen Reichsmuseums zu Stockholm beschäftigt ist, gehören in der Tat die von der Vega-Expedition am östlichsten Teil der sibirischen Küsto gesammelten Exemplare ohne Ausnahme zu $U$. coarctatus (ich habe selbst dio 'fiere gesehen; schon eino flüchtige Untersuchung zeigt, dass sio typischo $I I$. coarctatus sind). STUxBERG's $I$. araneus 1882 stellt also teilweise $I I$. coarctatus, seine $I I$. conretatus 1880 stollt wahrscheinlich $I I$. araneus dar.

I Brand crwähnt II. araneus aus dem Ochotskischen Meer (Doxs [1913] vermutet, dass auch seine $H$. conretalus var. alutacea zu araneus gohört; diese Ansicht wird durch dio Doss unbekannten Ausführungen BiruLA's [1910] widerlegt). Brrura (1910) hat eino Abbildung des BRArd'schen Originalexemplars (nur Carapax) reriffentlicht. Nach der Ausbildung des Postorbitalprozesses müsste man dieses Exemplar zur arktischen Form von II. araneus rechen. Yon allen späteren Expeditionen (North Pacific Exploring Expedition usw.) ist jedoch, wie Breves hervorhebt, in pazifischen Gebiet und nördlich davon überall nur 11 . latifrons STiMrs. gefunden worden, wolcho zu dem Formenkreis von $I I$. coaretatus gehört und als var. alutacea BRANDT zu bezeichnen ist; auch IRATIBUN (1904) crwïhnt nur dieso Art. Da der fragliche Brandr'sche Carapax, soweit ich sche, seiner Gesantform nach gut mit $I I$. conctatus übereinstimmt, kaun man dio Jöglichkeit nicht ausschliessen, dass es sich um ein ab. weichendes Exemplar dieser Art, also um eillo Konvergenzerscheinung handelt. Solango koine weiteren Fundo vorliegen, muss man jedenfalls das Vorkommen von $I$. arateus im pazifischen Gebiet als nicht sicher erwiesen bezeichnen. Das Gesagto gilt natürlich nur unter der Voraussetzung, dass es sich um zwei erblich gotrennto Species handelt, was vielleicht nicht sicher erwiesen ist. 


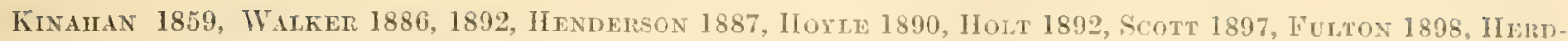

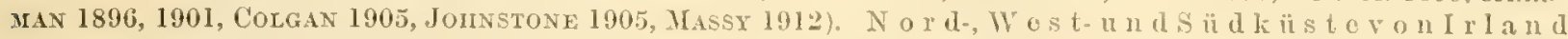
(THOMPSON 1842, 1844, 1856, BeLT 1853, ANDFETS 1878, IIOT, 1892).

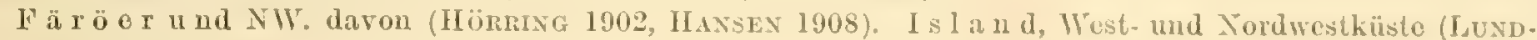
BECK 1893, HöRRING 1902, HANSEN 1908).

Bathymetrische Verbreitu $\mathrm{g}$. Tn der arktischen Region kann $H$. araneus bisweilen schon in der Uferzone auftreten, doch scheint er in der Regel überall, wie im Eisfjord, oberhalb von etwa $20 \mathrm{~m}$ sehr selten zu sein (ORTMANN 1901: 0 bis $2 \mathrm{~m}$; vereinzelte Funde 9-11 m: STUXBerg 1886, HANsen 1837, 1887 a und oben). Die grösste Tiefe beträgt $310 \mathrm{~m}$ (BIRULA 1907) oder nach RATHBUN $500 \mathrm{~m}$, schon unterhalb von $200 \mathrm{~m}$ ist die Art aber nur wenige mal gefunden worden.

In der borealen Region ist die Art schon in geringer Tiefe allgemein, und sie steigt auffallenderweise wenigstens in den meisten Gegenden nicht tief hinab; rgl. besonders APPelLöF 1906 ( in seichtem Wasser», „Hauptverbreitung in der ganzen litoralen liegion..., in deren oberen Abteilung häufig»), ferner HENDERSON 1887, SсотT 1888, Metzger 1874 (0 bis $2-47 \mathrm{~m}$ ), Meinert 1877 (4, $0-33 \mathrm{~m}$ ), Aurivillius 1889 (6-20 m), LAGERberg $1908(10-30 \mathrm{~m})$, Stephitensen $1910(2-30 \mathrm{~m})$. AppellöF (1. c., S. 160$)$ denkt sich, dass diese Art möglicherweise, wie einige andere Crustaceen, im Innern der Fjorde in grösserer Tiefe lebe, und fuir diese Möglichkeit können in der Tat einige Beobachtungen angeführt werden (SARS 1872: Hardangerfjord, vin etwas grösserer Tiefe»; GRIEG 1914: Hardangerfjord, $\gg 10-100 \mathrm{~m} \gg$; WoLLEBer 1900: Kristiania- und Breviksfjord, 90-108 m; Trribon 1903, Bлörск 1911: Gullmarfjord, 76 bis 107, 70 bis 100 m). Eine Erklärung der verschiedenen Lebensweise in den arktischen und in den borealen Gegenden (oder wenigstens deren offenen Küstenpartien) kann gegenwärtig schon deshalb nicht versucht werden, weil man nicht sicher weiss, ob das Tier in der arktischen Region überall die Uferzone meidet. (Die Annahme einer ökologischen Anpassung derselben Art, wie sie Spirontocaris gaimarlii aufweist, scheint wenigstens gegenwärtig auf grosse Schwierigkeiten zu stossen.) 


\section{Allgomeiner Teil. \\ Die Verbreitung der Decapoden im Eisfjord.}

\section{Artanzahl und Hiinfigkeit.}

9 Decapodenarten sind aus dem Eisfjord bekannt: Spirontocaris spinus, S. turgida, S. polaris, S. gaimardii, Sclerocangon boreas, Sabinea septemcarinata, Pandalus borealis, Eupagurus pubescens, Hyas araneus. Es ist nicht ausgeschlossen, dass in Zukunft einzelne andere Arten dort gefunden werden; dabei muss es sich jedoch um nur zufällige Gäste oder wenigstens äusserst seltene Tiere handeln. Unter den hocharktischen Arten könnte möglicherweise z. B. Sclerocrangon ferox, unter den arktisch-borealen Bythocaris simplicirostris vereinzelt und lokal im Eisfjord auftreten; rein oder vorwiegend boreale Arten, deren Vorkommen als zufällige Gäste nicht ganz unerwartet wäre, sind Sabinea sarsii und Hyas coarctatus.

Die 9 von uns gefundenen Arten sind sicher konstante Mitglieder der Eisfjordfauna. Sie sind alle mehr oder weniger allgemein. Weniger häufig als die übrigen ist Hyas arıneus (8 Fundorte), was jedoch teilweise damit zusammenhängt, dass diese Art vereinzelt lebt. Unter den ïbrigen sind Spirontocaris polaris, S. gaimardii, Sclerocrangon boreas und Sabinea septemcarinata besonders allgemein (40-44 Fundorte; im einzelnen verweise ich auf den Speziellen 'Teil). Die Natantia und Eupagurus pubescens treten, wie iiberall, oft in mehr oder weniger grosser Individuenzahl auf. In den grössten Scharen leben die 4 soeben erwähnten allgemeinsten Arten, ferner und vor allem Pandalus boreatis (welcher eigentlich ebenso häufig ist, obgleich das Gebiet des Vorkommens beschränkter ist).

\section{Einwirkng der Bodenbeschaftenheit.}

Die Abhängigkeit von der Bodenbeschaffenheit ist oben bei den einzelnen Arten erörtert worden; hier soll nur das Wichtigste zusammengefasst werden.

Zwei Arten, Pandalus boreatis und Sabinea septemcarinata, sind auf den reinen oder mit Steinen, Kies usw. gemischten Schlammgrumd beschränkt; für die erste hat diese Regel keine ganz allgemeine Gültigkeit, denn in andern Gegenden, wo härterer Boden in 
grösserer Tiefe vorhanden ist, kann sie während der Fortpflanzumgszeit auch auf solehem auftreten.

Die iibrigen Arten bevorzugen festeren Boden oder mit härterem Material gemischten Schlammgrund, sind aber gleichzeitig mehr ubiquistisch und kommen somit, obgleich mehr oder weniger selten, auch auf ungemischtem Schlammboden vor. Am wenigsten ubiquistisch ist Spirontocaris turgida, die fast nie auf losem ungemischtem Schlammgrund auftritt. Spirontocaris gaimardii, Sclerocrangon boreas, Euparurus pubescens und Hyas araneus kommen auf solchem vor, sind aber unvergleichlich häufiger auf mit Steinen oder Kies gemischtem Schlamm und härterem Boden. Spirontocaris spinus und besonders S. polaris sind häufiger als die iibrigen auf reinem Schlammgrund; anch sie bevorzugen jedoch gemischten oder härteren Grund.

Besonders häufig sind diese mehr oder weniger dentlich den harten Boden bevorzugenden Arten unter den Balanus porcatus-Gemeinschaften und unter Lithothamnien. Unter den Balaniden sind sie alle ausserordentlich häufig, Spirontocaris turgida und Sclerocrangon boreas vielleicht noch mehr als die übrigen. Auf Lithothamion-Grund scheint S. spinus verhältnismässig wenig häufig zu sein, die ïbrigen (ausser Hyas artneus?) sind dort ungefähr ebenso allgemein wie unter Balaniden.

Auf den verschiedenen Bodenarten findet man also folgende Decapoden:

Auf dem losen, ungem isehten Sch I a $\mathrm{mmgrund:}$ Pandulus borealis, Sabinea septemcarinata, sehr allgemein; Spirontocaris polaris, ziemlich häufig; S. spinus, ebenso oder eher etwas seltener; S. gaimardii, Sclerocrangon boreas, Euparyums pubescens, Hyas araneus, mehr oder weniger selten; S. turgida, äusserst selten.

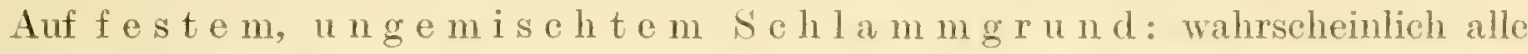
Arten; nur S. turgida wurde verhältnismässig häufig auf diesem wenig verbreiteten Boden gefunden.

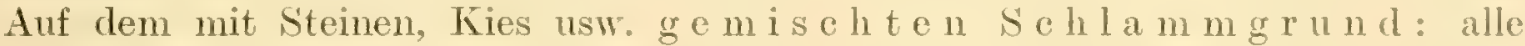
Arten, mehr oder weniger allgemein; am seltensten Pandalus borealis.

Auf dem mit Laminarien bewachsenen Steingrund: Nur Spirontocaris turgida, S. gaimardii, Sclerocrangon boreas und Hyas araneus, und anch sie sehr selten; diese Seltenheit beruht wenigstens zum grossen 'Teil darauf, dass solcher Boden fast nur in der Uferzone anzutreffen ist.

Auf $\mathrm{L}$ ith ot ha $\mathrm{m} n$ i on - G r und (wenigstens teilweise stranchförmiges Lithothamnion auf steiniger oder schlammiger Grundlage): Spirontocaris turgida, s. polaris. Sclerocrangon boreas, Eupagurus pubescens, äusserst allgemein; S. gaimardii, vielleicht nicht ganz so häufig; S. spinus (und wohl Hyas araneus), ziemlich allgemein; Sabinea septemcarinata, nur gelegentlich.

In den Balanus porcatus-Gemeinschaften: Spirontocaris turgida, Sclerocrangon boreas, S. gaimardii, Eupagums pubescens, äusserst allgemein; S. spinus, S. polaris und wohl Hyas araneus, annähernd ebenso allgemein; Sabinea septemcarinata und Pandalus boreatis, selten (wenigstens in der Regel nur bei Schlammboden), 
Einwirkung der flicte: Bbthymetrische Verbreitung der Decapoden im Eisfjord.

Die groben Züge der Vertikalverbreitung innerhalb des Eisfjords werden durch die Nachstehende graphische Darstellung veranschaulicht (Fig. 11).

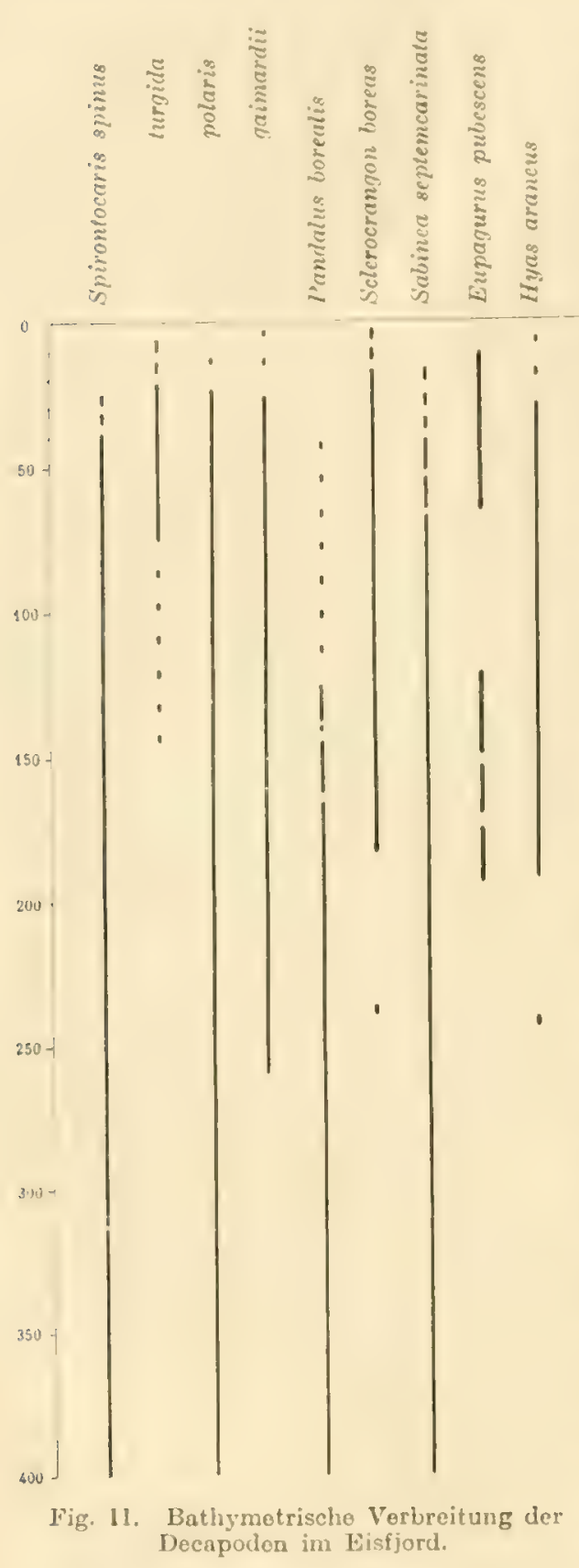

Ian kann zunächst konstatieren, dass alle Arten in der eigentlichen Uferzone mehr oder weniger selten sind, obgleich im einzelnen Unterschiede vorhanden sind. Spirontocaris turgida und Sclerocrangon boreas sind weniger selten als die übrigen zwischen 2 oder 5 und $20 \mathrm{~m}$, und der letztere ist schon von $15 \mathrm{~m}$ an ungefähr ebenso häufig wie weiter unten; Eupagurus pubescens fehlt zwar oberhalb von $10 \mathrm{~m}$, ist aber schon von dieser Tiefe oder wenigstens von $15 \mathrm{~m}$ an allgemein. Spirontocaris gaimardii, Hyas araneus und $S$. polaris treten bei 2, bzw. 6 und $10 \mathrm{~m}$ auf, sind aber bis in 20-25 m Tiefe äusserst selten. Sabinea septemcarinata fehlt ganz bis $15 \mathrm{~m}$, S. spinus bis $25 \mathrm{~m}$, Pandalus boreatis bis in noch grösserer Tiefe.

'Trotz der geringen Anzahl der Eisfjorddecapoden sind unter ihnen mehrere verschiedene Typen der Vertikalverbreitung vertreten; man kann folgende, teilweise gut getrennte, teilweise durch Übergangsformen verbundene Gruppen unterscheiden.

l. Seichtwasserart: Spirontocaris turgida (5- 140 oder 150 , meist oberhalb von $75 \mathrm{~m}$ ). 2. T i e f e $\mathrm{n}$ a $\mathrm{r} \mathrm{t}$ : Pandalus borealis (40, in der Regel $125-400 \mathrm{~m}$ ). Diese beiden Arten repräsentieren zwei Extreme. Es gibt zwar eine Zone, wo beide leben können, das häufige Vorkommen der einen begimnt aber erst weit unterhalb der eigentlichen Heimatzone der andern. Die Karte 1 zeigt, dass die Verbreitungsgebicte dieser Arten in der Regel vollständig getrennt sind.

3. Ne hr oder we niger e urybathe Art e n (in der Uferzone jedoch fehlend oder sehr selten): Spirontocaris polaris (10, in der Regel $20-$ $400 \mathrm{~m}$ ), S. spinus (25, meist 35 oder $40-400 \mathrm{~m})$, Sabinea septemcarinata (15, meist $40-400 \mathrm{~m})$. Die beiden letzteren Arten nähern sich, wie man sieht, ein wenig den 'Tiefenarten, S. spimus wohl auch der folgenden Abteilung (wie es scheint etwas seltener in der grössten 'Tiefe).

4. Die iibrigen Decapoden nehmen eine Art Zwischenstellung zwischen der 1. und 3. Gruppe ein. Sie sind allgemein bis in bedentend grössere Tiefe als Spirontocaris tur- 
gida, fehlen aber ganz in der Fjordtiefe. Hieher gehören: Spirontocaris gaimardii (2, in der Regel $25-260 \mathrm{~m}$ ), Sclerocrangon boreas (2, öfter 15 - 185, ausnahmsweise $230 \mathrm{~m}$ oder etwas mehr), Hyas araneus $(6$, meist $30-185$, ausnahmsweise $240 \mathrm{~m})$, Eupagurus pubescens $(10$ - nahezu $200 \mathrm{~m})$. Die drei erstgenannten Arten steigen auch in andern Gegenden nicht wesentlich tiefer als im Eisford hinab, Eupagurus pubescens dagegen nimmt in zwei Hinsichten eine Sonderstellung ein. Erstens ist diese Art in andern Gegenden eurybather als irgend eine der anderen Arten; die beschränkte Vertikalverbreitung im Eisfjord muss daher ganz spezielle Ursachen haben. Zweitens weist die Verbreitung eine eigentümliche, wahrscheinlich von den Temperaturverhältnissen bedingte Lüicke zwischen 65 und $120 \mathrm{~m}$ auf.

Ihrer allgemeinen Vertikalverbreitung nach sind die meisten Eisfjorddecapoden ausgesprochene Flachseearten, höchstens bis in $300-450 \mathrm{~m}$ hinabsteigend und damn seltener schon unterhalb von 200-300 oder sogar (Spironcaris turgida) $75 \mathrm{~m}$. Spirontocaris polaris und Eupagums pubescens sind mehr eurybath, ohne jedoch in grosse Tiefen hinabzusteigen (bis etwa $900 \mathrm{bzw} .1100 \mathrm{~m}$, wohl schon von etwa $500 \mathrm{~m}$ an Häufigkeit abnehmend). Pandalus borealis ist auch ziemlich eurybath, das Gebiet des häufigen Vorkommens ist jedoch sowohl nach oben wie nach unten hin beschränkter.

Eine Einteilung des Eisfjords in Vertikalzonen nach der.Verbreitung der wenigen decapoden Crustaceen hat natürlich keinen Zweck. Man würde jedenfalls nur zwei, durch ein breites Übergangsgebiet ineinander greifende Zonen unterscheiden kömen. Die untere ist vorwiegend durch negative Charaktere, positiv nur durch das Vorkommen von Pandalus borealis ausgezeichnet. - Zwischen etwa 40 und $70 \mathrm{~m}$ findet man alle 9 Arten, die letzterwähnte jedoch selten. Nach unten zu wird die Artanzahl bald gering; in der Tiefenhöhle am Eingang des Fjords (Svensksundstiefe), von etwa 250 bis $400 \mathrm{~m}$, leben nur 4 Arten.

\section{Einwirkung der Wassertemperatur.}

Die Abhängigkeit von den Temperaturverhältnissen oder mit andern Worten die Thermopathie der Eisfjorddecapoden hängt imig mit ihrer allgemeinen Verbreitung und tiergeographischen Stellung zusammen, und ich will hier nur eine gedrängte Übersicht der Arten geben. In bezug auf die allgemeinen Gesichtspunkte bei der Behandlung dieser Fragen verweise ich auf meine Arbeit iiber die Echinodermen des Lisfjords.

Wenn die im Eisfjord beobachteten Verhältnisse ein wahrer Ausdruck der Thermopathie der Arten wäre, so wïrden die meisten Eisfjorddecapoden 'Iemperaturen zwischen +3 und -1 oder $0^{\circ}$ bevorzugen und sowohl das wärmste wie das kälteste Wasser mehr oder weniger deutlich meiden. Ich habe oben unter den einzelnen Arten nachgewiesen, dass eine solche Betrachtungsweise ganz falsch wäre; weder das wärmste noch das kälteste Wasser ist in jeder Tiefe und auf jedem Boden zu finden; man muss in jedem Fall untersuchen, ob das Fehlen unter gewissen 'Temperaturen dureh die 'Temperatur oder durch andere Faktoren bedingt wird. Die Resultate der Untersuchungen lieriber stelle ich unten zusammen; die Unsicherheit, welche wegen der Unvollstïndiglieit des Tatsachenmaterials in mehreren Fällen den Schlussfolgerungen anhaftet, kann hier we- 
niger deutlich als in den speziellen Darstellungen zum Vorschein kommen. Unter oberer I'emperaturgrenze verstehe ich das Sommermaximum; ein tieferes Eindringen in die Natur der Temperatureinwirkungen kann gegenwärtig nicht versucht werden (vgl. meine soeben erwähnte Arbeit).

Verhältnismässig leicht gelingt der Nachweis, dass die Seltenheit im kältesten Wasser in bezug anf 5 Arten nichts mit der niedrigen 'lemperatur zu tun hat, sondern durch andere Umstände, vor allem die Tiefe und die Bodenbeschaffenheit, hervorgerufen wird; diese Arten sind die vier Spirontocaris-Arten und Sabinea septemcarinata. Zu ihnen gesellt sich Sclerocrangon boreas, der auch im Eisfjord in Wasser von negativer Temperatur häufig ist, obgleich er nicht unterhalb von $-1^{\circ}$ gefunden wurde.

Fiir Pandalus borealis, Eupagurus pubescens und Hyas araneus kann dagegen mit mehr oder weniger grosser Sicherheit festgestellt werden, dass sie wirklich Wasser von negativer 'Temperatur meiden.

In bezug auf die ho hen 'Temperaturen liegen die Dinge verwickelter, weil hier so viele und nicht immer gut bekannte Umstånde in Betracht zu ziehen sind. Wiir Spirontocaris polaris kann mit grosser Wahrseheinlichkeit nachgewiesen werden, dass die im Eisfjord konstatierten Verhältnisse (sie wurde ausnahmsweise zwischen + 3 und $+4^{\circ}$, gar nicht in noeh wärmerem Wasser gefunden) die Thermopathie der Art rich. tig wieclergeben; die obere 'Temperaturgrenze liegt also in der arktischen Region bei etwa + 3०. Mehrere 'Tatsachen erlauben die Vermutung, dass sich Spirontocaris turgida und vielleicht auch S. gaimardii ähnlich verhalten. Ob auch S. spinus und Sabinea septemcarinata zu dieser Gruppe gehören, muss dagegen offen gelassen werden. Sclerocrangon boreas erträgt eine Sommertemperatur von wenigstens $+5^{\circ}$.

Pandalus boreatis, Eupagurus pubescens und Hyas araneus fehlen im wärmsten Wasser des Fjords, eine Einwirkung der 'Temperatur ist jedoch ausgeschlossen; sie ertragen sonst noch höhere 'Temperaturen.

Von allen Einzelheiten abgesehen, kann man nach dem oben Gesagten im Eisfjord zwei Gruppen unterscheiden, welche in ihrer allgemeinen Verbreitung und ihrem Anpassungsvermögen wenig einheitlich, unter arktischen Bedingungen jedoch als zwei dentlich getrennte Kategorien erscheinen: 1. Arktisch-eurytherme Kaltwasserarten, in Wasser von negativer und niedrig positiver 'Temperatur lebend ('Temperaturamplitude also Jinimum des Seewassers - etwa $+3^{\circ}$ oder etwas mehr): Spirontocaris spinus, S. turgida, S. polaris, S. gaimardii, Sabinea septemcarinata, Sclerocrangon boreas (die letztere Art und vielleicht einige andere ein Maximum von $+5^{\circ}$ ertragend und daher eine weniger ausgeprägte Kälteart als die übrigen; $S$. gaimarlii möglicherweise zu einer dritten Gruppe von noch eurythermeren Arten gehörend). 2. Warmwasserarten, vorwiegend in Wasser von positiver T'emperatur lebend: Pundalus boreatis (verhältnismïssig stenotherm, 'Temperaturamplitude etwa $0-+6^{\circ}$ bis $7^{\circ}$ ); Eupagurus pubescens und Hyas araneus (eurythermer, obere T'emperaturgrenze höher).

Der Linfluss der Temperaturverhältnisse auf die Verbreitung im Eisfjord wird in einem folgenden Absehnitt besprochen. Die nötigen Mitteilungen iber die Hydrographie des Fjords habe ich in meiner Arbeit über die Echinodermen zusammengestellt; rol. atuh die Hydrographischen Ergebnisse der Expedition (Nir. Hydrog. Kommiss. 1910). 


\section{Übrige Faktoren.}

Über die Abhängigkeit vom S a I z g e ha $1 \mathrm{t}$ des Wassers kann wenig gesagt werden, da aus den meisten Gegenden keine in dieser Hinsicht verwertbaren Beobachtungen vorliegen.

Mehrere der Eisfjorddecapoden sind jedenfalls ausgesprochen e u r y ha 1 in und ertragen Schwankungen des Salzgehalts von mehr als 34 bis 25 oder $20 \%$. Solche Arten sind Spirontocaris gaimardii und Hyas araneus (besonders nach der Verbreitung im Kattegatt und benachbarten Gewässern), ferner wahrscheinlich Sclerocrangon boreas (nach der Verbreitung im Eisfjord zu urteilen), nach einigen Beobachtungen der VegaExpedition (STUxberg 1882) auch Sabinea septemcarinata (im Eisfjord nur in Wasser von mehr als $34 \%$ Salzgehalt).

Spirontocaris spinus und $S$. polaris leben im Eisfjord und in vielen Gegenden die erstere wie es scheint überall - ausschliesslich in Wasser von mehr als $34 \%$ Salzgehalt; dass sie von diesem hohen Salzgehalt abhängig sind, kann jedoch nicht erwiesen werden. Spirontocaris turgida und Eupagurus pubescens scheinen einen Salzgehalt von 32 bis $33 \%$ zu ertragen und sind also jedenfalls nicht sehr stenohalin; weitere Schlussfolgerungen sind nicht möglich.

Pandalus borealis ist in der Regel uiberall, wie im Eisfjord, nur in Wasser von wenig wechselndem Salzgehalt (34 oder wenigstens $33,75-35 \%$ ) beobachtet worden (s. besonders WOLLEBæK 1908). BJörck (1911, 1913) hat indessen in einzelnen sehwedischen Fjorden diese Art, teilweise in beträchtlicher Menge, in Wasser von bloss 28,39 -29\% Salzgehalt gefunden. Diese Beobachtungen scheinen ja darauf hinzuweisen, dass die in der borealen Region konstatierte Beschränkung auf spezielle Wasserschichten vorwiegend durch die Temperatur bewirkt wird; das charakteristische Auftreten der skandinavischen Küste entlang macht es jedoch wahrscheinlich, dass die Art eine starke Herabsetzung des Salzgehalts in der Regel nicht erträgt. Von grossem Intresse ist, dass sie im atlantischen Wasser der Sliageraktiefe, mit seinem Salzgehalt von mehr als $35 \%$, so gut wie ausnahmslos fehlt (s. WOLLEBEK; BJöRCK); nach STEPHENSEN (1910) lebt sie allgemein "fast überall im tiefen Skagerak», in 52-570 m Tiefe; sowohl die Beobachtungen der soeben erwähnten Autoren, wie die von Petersen \& Levinsen (1900) veröffentlichten Funde zeigen jedoch, dass die Art in der Regel nur bis in die oberen. Grenzschichten des atlantischen Wassers hinabsteigt; die letzteren Autoren verzeichnen mehrere Fundorte mit vielen Exemplaren bis in 190 m Tiefe, 2 Fundorte in etwa $230 \mathrm{~m}$ Tiefe, 1 Fundort mit wenigen Exemplaren in etwa $375 \mathrm{~m}$ Tiefe). Es ist nicht mwahrscheinlich, dass die Art einen Salzgehalt von mehr als $35 \%$ direlit meidet, doch ist es naturlich denkbar, dass das Fehlen in der Skageraktiefe eher mit der Herkunft als mit der Beschaffenheit des Wassers zusammenhängt. Erst genaue Untersuchungen iiber die Lebensbedingungen in andern Gegenden können hieriber Aufschluss bringen.

Die Salzgehaltschwankungen üben zweifellos keinen merkbaren Einfluss auf die Verbreitung der Arten in Fisford aus. Wasser mit einem Salzgehalt von $34 \%$ findet sich im ganzen Fjord schon in mehr oder weniger umbedentender Tiefe. Auch die Verti- 
kalverbreitung der wahrscheinlich am meisten stenohalinen Art, Pandalus boreatis, wird nicht durch den Salzgehalt beeinflusst; sie tritt in der Regel erst weit unterhalb der oberen Grenze des $34 \%$-Wassers auf.

Hinsichtlich anderer Faktoren, welche auf die Verbreitung im Fisford einen - in Einzelheiten wahrscheinlich wichtigen, doch kaum durchgreifenden - Einfluss haben mögen, kann man nur auf die Möglichkeit ihrer Existenz aufmerksam machen; vgl. "Die Echinodermen des Lisfjords", S. $192 \mathrm{ff}$.

\section{Zusammenwirken der Faktoren Bodenbeschaffenheit, Thefe mol Wassertemperatur: das Verbreitungsbild der Decapoden im Kistjord.}

Unter den Faktoren, durch deren Zusammenwirken jede Art ein charakteristisches Verbreitungsbild im Eisfjord erhält, ist der ohne Frage wichtigste die 'Tiefe oder, richtiger gesagt, die meist unbekannten Faktoren, welche die Vertikalverbreitung bestimmen. Die Bodenbeschaffenheit ist von grösserer Bedentung nur in bezug auf an harten Grund gebundene Tiere, welche die grossen Schlammebenen der zentralen Fjordpartien meiden müssen; sowohl die Schlammarten wie die von der Bodenbeschaffenheit mehr oder weniger unabhängigen Tiere finden in der ganzen horizontalen und vertikalen Ausdehnung des Fjords giinstigen Grund, obgleich die Verbreitung der ersteren natülich in seichtem Wasser stellenweise unterbrochen ist. Die Wassertemperatur vermag in einigen Fällen die Vertikalverbreitung zu modifizieren; eine Einwirkung auf die horizontale Verbreitung ist bei den Warmwasserarten vorhanden oder denkbar.

Spirontocaris polaris und S. spinus sind mehr oder weniger eurybath und eurytherm, obgleich wenigstens die erstere Wasser von mehr als $+3^{\circ}$ Sommertemperatur meidet, und an keinen besonderen Grund gebunden, obgleich spärlicher an reinem losem Schlammboden. Sabinea septemcarinata ist eine Schlammart, stimmt aber sonst mit den erwähnten Arten iberein. Wie diese Voraussetzungen erwarten lassen, sind diese drei Arten ii ber den ganzen F jord verbreitet (Karte 3,4). Alle drei fehlen jedoch ganz oder fast ganz in seichtem Wasser, die beiden ersteren oberhalb von etwa $25, S$. septemearinala oberhalb von $15 \mathrm{~m}$. Eine Folge davon ist, dass sie in einer schmalendie letzterwähnte Art in einer ganz schmalen - Randzone fehlen. Hieraus erklärt sich auch das Fehlen im grössten Teil der seichteren Baien, 'Tundra, Yoldia, Ekman und Coles Bay (wahrscheinlich dringen sie etwas weiter in diese Buchten ein, als es unsere Beobachtungen an die Hand geben; das vollständige Fehlen in der Tundra und Yoldia Bay erklärt sich z. B. daraus, dass wir dort nur Stellen in der Uferzone oder mit ungeeignetem Boden untersuchten). Einige Unregelmässigkeiten in der Verbreitung werden leicht verständlich, wenn man die Bodenbeschaffenheit und die ungleiche Verteilung der untersuchten Stationen berücksichtigt. In der Dickson Bay und im grössten T'eil der Advent Bay wurde nur S. septemcarinala angetroffen; in der ersteren scheint der Grund iiberall aus reinem, rotem Schlamm zu bestehen - die beiden Spirontocaris-Arten dürften daher sicher selten sein, wenn sie auch vielleicht nicht fehlen -; in der Advent Bay untersuchten wir (ausser am Eingang) nur drei Stellen, davon zwei in seichtem Wasser, eine mit losem Schlammboden. S. spinus wurde auch in der Ymer und in der Tempel Bay ver- 
misst, obgleich wir dort zahlreiche Dredgungen ausfiihrten; die meisten Stationen liegen jedoch oberhalb der oberen Verbreitungsgrenze der Art oder in einer Zone (25-40 m), wo sie im Gegensatz zu den beiden anderen Arten noch selten ist; auf dem roten Schlammgrund der Tempel Bay sind übrigens alle Decapoden selten.

Spirontocaris gaimardii unterscheidet sich von S. polaris daclurch, dass sie nicht bis in die grösste Tiefe des l'jords hinabsteigt, obgleich sie noch in den tieferen Partien des Fjordstamms, bis zu etwa $260 \mathrm{~m}$, nicht selten ist. Das Verbreitungsbild unterscheidet sich demnach von demjenigen der vorigen Arten hauptsächlich durch das ti e h 1 e $n$ in der Tiefenhöhle a mingang des F Fords (Karte 4).

Spirontocaris turgida (Karte 1), Sclerocrangon boreas (Karte 2), Eupagurus pubescens

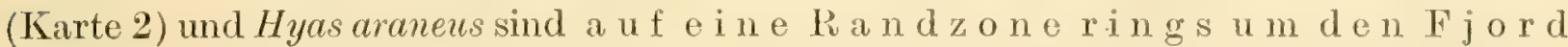
beschränkt und fehlen vollständig in der ganzen zentralen Partie des Fjordstammes und der Hauptarme. Am schmälsten ist diese Zone bei S. turgida, welche hauptsächlich oberhalb von der $75 \mathrm{~m}$ Kurve lebt; bei den übrigen, bis zu beinahe $200 \mathrm{~m}$ (ausnahmsweise etwas mehr) hinabsteigenden Arten ist sie breiter. Der Unterschied in dem Verbreitungsbild ist jedoch verhältnismässig umbedeutend, weil die Fjordabhänge meist sehr steil sind. Die Ursache dieser beschränkten Verbreitung ist etwas verschieden. Eupagurus pubescens steigt in andern Gegenden nicht selten tiefer als bis in die grösste Tiefe des Eisfjords hinab; das Fehlen in den unteren Teilen des Fjords beruht zweifellos hauptsächlich darauf, dass die Art den remen losen Schlammboden meidet. Die drei uibrigen sind uiberall nur in mehr oder weniger seichtem Wasser zu finden; ihre beschränkte Verbreitung im Eisfjord ist demnach wenigstens bis zu einem gewissen Grade unabhängig von der Bodenbeschaffenheit.

Sowohl Eupagurus pubescens wie Hyas araneus sind Warmwasserarten, und man könnte erwarten, ihre horizontale Verbreitung im Eisfjord dadurch beeinträchtigt zu finden. Beide Arten kommen jedoch sowohl in den inneren wie in den äusseren Teilen des Fjords vor, was eigentlich nicht allzu uiberraschend ist, da sie keine ganz strengen Warmwassertiere sind, nur rein hocharktische Bedingungen meiden. $H$. araneus ist ïbrigens nur einmal im kalten Gebiet angetroffen worden; obgleich die geringe Anzahl der Fundorte keine sicheren Schliisse erlaubt, ist es sehr möglich, dass er uiberwiegend in den wärmeren Teilen des Fjords zu Hause ist.

Die Verbreitung von $E$. pubescens wird wahrscheinlich von den Temperaturverhältnissen beeinflusst, obgleich dies nur undeutlich im horizontalen Verbreitungsbild zum Ausdruck kommt. Wie ich im Speziellen Teil nachgewiesen habe, meidet sie die intermediäre Kaltwasserschicht; die Fundorte in den inneren Teilen des Fijords, wo die kalte Schicht bis auf den Grund hinabreicht, liegen alle in seichtem, im Sommer mehr oder weniger warmem Wasser (Temperatur mit einer Ausnahme $+\mathbf{1 , 5}$ bis $\mathbf{2}^{\circ}$ oder mehr).

Pandalus borealis ist eine Schlamm- und Tiefenart, die Verbreitung daher a uf d e n tiefen zentralen Teil des Fjords sowie auf die tieferen Baien be-

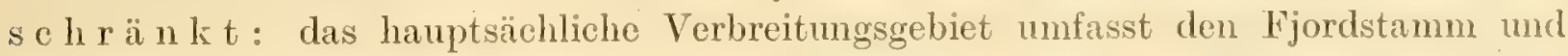
die beiden Hauptarme (im Ostarm nur am Eingang gefunden, zweifellos nur wegen ungenügender Erforschung dieses Gebietes). Diese Art steht also in ausgesprochenem Gegensatze zu den zuletzt besprochenen Arten, vor allem zu Spirontocaris turgida (Karte 
1). In den inneren, kältesten Baien wurden nur zwei Exemplare gefangen; obgleich die Beweiskraft dieser Tatsache durch die beschränkte Tiefenverbreitung beeinträchtigt wird, lamn man nicht daran zweifeh, dass diese Art - in ihrer ganzen Verbreitung eine deutliche Warmwasserart - vorwiegend dem wa men 'I'iefen wasser der äuseren und mittleren Fjordpartien angehört.

Die oben zusammengestellten 'Tatsachen zeigen, dass das Verbreitungsbild der Irten im lisf jord, wie schon anfangs betont wurde, hauptsächlich eine Wiederspiegelung der Vertikalverbreitung ist, welche ihrerseits durch die Bodenbeschaffenheit (Ëupagurus pubescens) und dureh die Wassertemperatur (wenigstens Spirontocaris polaris und $E$. pubescens, bei diesen im entgegengesetzten Sime) beeinflusst werden kann. Nur bei Pandalus boreatis haben die Temperaturverhältnisse einen deutlichen Einfluss auf die horizontale Verbreitung.

\section{Allgemeine Verbreitung der Decapoden der arktischen Region.}

\section{Historischer Überhlick.}

Whe ich meine eigenen Ansichten darlege, will ich die fribere Literatur über die Verbreitung der arktischen und nordischen Decapoden besprechen; ich berícksichtige dabei nur diese Tiere, ohne auf die Entwicklung der ganzen marinen Tiergeographie einzugehen.

Die älteste Literatur (DANA u. a.) hat nur noch historisches Interesse. Der erste Versuch einer speziellen. Behandlung der arktischen Malakostraken stammt von lionsSTRAND (1886); er versucht, die Verbreitung mit den physikalischen Bedingungen in 'Zusammenhang zu bringen, im einzelnen ist aber seine Arbeit jetzt veraltet.

Zehn Jahre später veröffentlichte ORTMANN (1896) unter besonderer Berïcksichtigtıng der decapoden Crustaceen seine Arbeit "Grundziige der marinen Tiergeographie». Dieses Buch enthält interessante, aber stets ganz allgemein gehaltene Erwägungen und hat wohl kaum einen Einfluss auf die Entwicklung der modernen, empirischen Tiergeographie ausgeibt; ich brauche es nicht näher zu berïcksichtigen, weil gar keine einzige arktische Art erwähnt wird. In seiner Nomenklatur verlässt OrTuans in unmotivierter Weise den frihheren tiergeographischen Sprachgebrauch und bezeichnet die ganze nördliche Hemisphäre nördlich der »circumtropischen Zone» als »arktisch». In dieser arktischen Region unterscheidet er eine meigentliche» arktische Subregion, deren Grenzen lediglich nach den physikalischen Verhältnissen, ohne Beriicksichtigung der 'Tierwelt, gezogen werden. Zu welchen Ergebnissen diese Methode fuhren kam, zeigen die Ausfuihrungen dieses Autors in seiner Crustaceenbearbeitung für Bronns »Klassen und Ordnumgen» (1901 a). Er bespricht hier auch die Verbreitung der Arten und glaubt feststellen zu kïnnen, dasss die meigentlichen arktische Regrion sich hinsichtlich der Decapoden nur durch den Mangel gewisser Formen auszeichnet; alle Arten (z. B. Sclerocrangon boreas, Sabinea septemcarinala, die Spirontocaris-Arten) »dringen auch mehr oder weniger weit ins boreale Gebiet ein und wir kömmen sie somit als charakteristisch für die ganze ark- 
tische Region bezeichnen 》. Dieser schwere Irrtum ist natürlich dadurch entstanden, dass der Autor zuerst die Suidgrenze der neigentlichen" arktischen Region bestimmt und nachher erst die Verbreitung der Arten untersucht hat. Die Grenze ist offenbar ganz willkürlich gezogen worden; der Verfasser hätte ohne allzu grosse Schwicrigkeiten finden können, dass die psysikalischen Bedingungen in Ostfinnmarken keineswegs boreal sind. Dofleis's Bearbeitung der Decapoden Krebse in der "Fauna arctica» (1900) hätte eine wichtige Hilfsquelle der heutigen Forschung werden können, wenn der Autor uur mit einiger Kritik ans Werk gegangen wäre. Dies ist leider nicht der Fall; wenigstens in vielen Hinsichten muss man das harte Urteil HANSEN's (1908) untersehreiben, dass die ganze Arbeit "almost quite useless» ist. Unter "die sicheren und im arktischen Gebiet nachgewiesenen Arten" wird eine ganze Reihe typisch borealer Formen aufgenommen; von tiergeographischen Erörterungen enthält die Arbeit nur einige Bemerkungen über die Verbreitung im Spitzbergengebiet, und diese sind vollständig irrig (s. unten S.98). Die Angaben iiber die Verbreitung der Arten sind oft sehr allgemein gehalten oder wegen der unkritischen Gutnahme aller älteren Angaben unrichtig.

Wenn man auch früh eingesehen hat, dass die Verbreitung der Decapoden der nördlichen Meere ein für die tiergeographische Forschung wichtiges und dankbares Thema darstellt, so wurde jedoch erst vor wenigen Jahren diese Tatsache vollauf und von modernen Gesichtspunkten aus verwertet. Dies gesehah in APPELLör's Arbeit „Die decapoden Crustaceen" (des Nordmeeres) (1906); in einer ein Jahr friher erschienenen Schrift allgemeinen Tnhalts (1905) werden dieselben Anschauungen entwickelt. In dieser Arbeit wird eine tiergeographisch-öliologische Analyse der Decapodenfauna des ganzen Nordmeeres geliefert; daraus resultiert eine auch für andere Gruppen gïltige Einteilung der nördlichen Meere in eine arktische Region, eine boreale Region und ein boreoarktisches Übergangsgebiet. Die Prinzipien waren wohl teilweise nicht neu, es ist aber ein sehr grosses Verdienst APpeluöF's, zuerst eine vollständige Bearbeitung einer Tiergruppe von solchen Gesichtspunkten aus durchfiuhrt zu haben.

Fast gleichzeitig und jedenfalls ohne Kenntnis von AppeluöF's Arbeiten veröffentlichte Birula (1907) eine tiergeographische Übersicht der Decapodenfauna von Spitzbergen. Er gibt eine gute Schilderung der hydrographischen und sonstigen äusseren Verhältnisse und trennt scharf das boreale Element von der in Spitzbergen heimischen Fauna, macht aber denselben Grundfehler wie Ortmann und findet, dass die arktische Region, was die Decapodenfauna betrifft, nur negativ charakterisiert ist.

Sehr wichtig ist die Arbeit HANSEN's (1908) iiber die Crustacea malacostraca der Ingolf-Expedition, vor allem wegen der kritischen Schärfe, die sowohl die systematischen Darlegungen wie die Ủbersichte der Verbreitung der einzelnen Arten auszeichnet. Die rein tiergeographischen Erörterungen beschränken sich jedoch auf eine Kritik der Arbeit DOFLEIN'S.

Eine ebenfalls wichtige Arbeit ist S'TEPHenses's Bearbeitung der Malacostraca der Danmark-Expedition nach Nordostgrönland (1912), welche eine cingehende tiergeographische Übersicht der Malakostraken von Grönland enthält (eine liüzere Zusammenstellung mit einzelnen Berichtigungen bei STEPHENSEN 1913). Die von diesem Autor aufgestellten Gruppen finde ich jedoch teilweise wenig gliicklich.. Ebenso richtig die 
Angaben ïber die einzelnen Arten sind, ebenso schematisch und unnaturlich ist ihre Verwertung zu tiergeographischen Schlussfolgerungen. So werden so ungleichwertige Elemente wie Hyas araneus, Eupagurus pubescens, Sabinea sarsii, Spirontocaris gaimardii, spinus und turgida zu einer gemeinsamen »boreoarktischen» Gruppe vereinigt; diese Gruppe umfasst ja rein arktische, hauptsächlich arktische, rein boreale und arktisch-boreale Arten. STEPHexsen legt in seiner Ubersicht das Hauptgewicht auf die Unterschiede in der bathymetrischen und ostwestlichen Verbreitung; wenn man zu einem wirklichen Verständnis der tiergeographischen Beziehungen der Tiere gelangen will, muss man diese Untersehiede mit den ron diesem Gesichtspunkte aus grundlegenden Unterschieden in der Abhängigkeit von den Temperaturbedingungen verknüpfen.

\section{Die nord-siidliche Verbreitung der Nordmeerarten.}

In ilırer Fähigkeit, verschiedene 'Iemperaturverhältnisse zu ertragen, oder mit andern Worten in ihrer Thermopathie zeigen die im arktischen Gebiet vorkommenden Decapoden beträchtliche Unterschiede. Es kamn nicht bezweifelt werden, dass die Nordund Siidgrenzen - diese Ausdriicke sind wegen der Bodenkonfiguration und der verwickelten hydrographischen Bedingungen der Meere nicht ganz adäquat aber jedenfalls verständlich - in der Regel durch diese Unterschiede bestimmt werden. In der Anwendung dieses Prinzips ist man jedoch nicht weit gekommen. Auch ApPeLtöf muss sich damit begnïgen, die zwei Hauptgruppen arktische und arktisch-boreale Arten zu unterscheiden, wemn er auch betont und durch Beispiele erläutert, dass weder diese noeh jene einheitlich ist.

Line genane Kenntnis der Lebensweise und der Verbreitung der Tiere wird uns in den Stand setzen, für jede Art bestimmte physikalische Bedingungen nachzuweisen, die, bald innerhalb enger, bald immerhalb weiter Grenzen schwankend, für das Leben erforderlich sind und die Verbreitung reglieren. Ich hatte die Hoffnung gehegt, aus dem Eisfjord ein genuigendes Beobachtungsmaterial zusammenzubringen, um in diese Richtung ein Stück vorwärts zu kommen. Allein diese Hoffnung ist nur in geringem Masse erfüllt worden; unsere Beobachtungen sind zu lückenhaft, die zum Vergleich nötigen Beobachtungen aus andern Gegenden ganz ungenügend. 'Irotzdem habe ich meinen Plan einer Detailuntersuchung über die Verbreitung der arktischen und arktisch-borealen Decapoden des Nordmeeres verfolgt, in der Neinung, dass es nützlich sein werde, sich klar zu machen, wi e weit man jetzt kommen kamn. In bezug auf die von mir gebrauchte tiergeographische Nomenklatur verweise ich auf meine Arbeit über die Echinodermen des Eisfjords. Auch die dort entwickelten allgemeinen Gesichtspunkte kann ich natürlich hier nicht wiederholen, ebensowenig die Vorteile und Begrenzung einer Einteilung in gesonderte tiergeographische Gruppen darlegen. Nur muss ich betonen, dass ich selbstverständlich die grosse Bedeutung nicht ïbersehen habe, welche rein biologisehe Verhältnisse, vor allem Unterschiede in der Fortpflanzung, für die Auffassung der tiergeographischen Stellung der Arten haben. Solange nichts hierüber bekannt ist, muss man sich jedoch damit begnügen, einfach von einem "Leben» in Wasser von gewissen Tempera- 
turen und einer allgemeinen Abhängigkeit von diesen Bedingungen zu sprechen. In jedem Falle auf alle denkbaren Komplikationen und Fehlerquellen aufmerksam zu machen. würde nur die Darstellung umnützerweise belasten.

Ich berïcksichtige unten auch die nicht vom Eisfjord bekamnten Nordmeerarten, das Hauptgewicht wird jedoch auf die Eisfjorddecapoden gelegt. Pelagische Formen

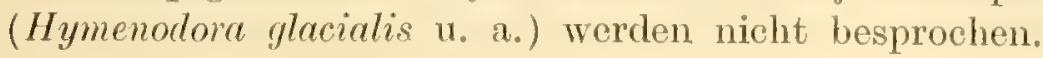

\section{Übersicht.}

\section{Arktische Arten.}

Wenn ich die in der arktischen Region heimischen Arten in die zwei Hauptgruppen arktische und arktisch-boreale Arten einteile, so muss ich gleich anfangs betonen, dass diese Gruppen bei den Decapoden nicht scharf von cinander getrennt sind, weniger scharf als z. B. bei den Echinodermen. Doch scheint es mir wenigstens der Übersichtlichkeit, halber besser, diese Hauptgruppen beizubehalten, als etwa bloss kleine, einheitliche ökologisch-geographische Gruppen zu unterscheiden. Ich rechne dabei einige in der borealen Region selten vorkommende, vorwiegend arktische Arten zur arktischen Gruppe.

\section{Hocharktische Arten.}

Hocharktische, d. h. an Wasser von konstant negativer Temperatur gebunderie Decapoden existieren im Eisfjord nicht. In der ganzen arktischen Pegion findet man nur drei solche: Bythocaris leucopis G. O. SARS, Bythocaris payeri (HELLER), Sclerocrangon ferox G. O. SARS.

Bythocaris leucopis ist, wie APPELLör hervorhebt, eine typisch ho e h a r k t is e ha b ys s a l e Art; sie ist auf die Nordmeertiefe beschränt und lebt dort in Tiefen von etwa $900-2860 \mathrm{~m}$, also ausnahmslos in Wasser von konstant negativer Temperatur (s. HANSEN 1908).

Bythocaris payeri und Sclerocrangon ferox werden von APPELLör zu einer besonderen Gruppe von »echten Kaltwasserformen » zusammengestellt; sie bewohnen die obere Region der kalten Area des Nordmeerbeckens, „ygleichzeitig aber auch die grösseren Tiefen der arktischen Plateaus" und können dort gelegentlich in Wasser von positiver Temperatur vorkommen, obgleich nur iauf der Grenze zwischen einem wärmeren und kälteren Stromgebiet». Die Verbreitung dieser Arten zeigt in der Tat unzweidentig, dass sie hocharktisch sind. B. payeri lebt in der Nordmeertiefe, ferner an der Kiiste von Nordostgrönland, im nördlichen 'I'eil des Barentsmeeres (1 Fundort) und bei Franz Josephs Land, in Tiefen von 182-1977 m; sie ist nur zweimal in Wasser von sehr niedrig positiver Temperatur $\left(+0,8,+1,5^{\circ}\right)$ gefunden worden, beidemal nahe an der Grenze von kälteren Wasserschichten (s. Appeldöf 1906, Hansen 1908, Stephensex 1913). S. ferox: lebt in demselben Gebiet wie die vorige Art (allgemein im Barentsmeer, vorwiegend jedoch im nördlichen 'Teil, auch die Fundorte im südlichen 'Teil in Wasser von negativer' Temperatur), ausserdem östlich davon im Karischen Meer und westlich davon in der Baffin Bay. Die Tiefenverbreitung erstreckt sich von etwa 90-1000 m (in grösserer T'iefe als $839 \mathrm{~m}$ nur 1 Exemplar gefunden); diese Art ist also überhaupt keine mechte 
'Tiefseeform, welche nur östlich im Kaltwassergebiet etwas aufsteigt» (BIRULA), sondern hat ihre Heimat im hocharktischen Küstengebiet, nur die obere Zone rom Ufer bis etwa $100 \mathrm{~m}$ meidend, und im obersten 'I'eil der Tiefenarea (s. Appellör 1906, BIRULA 1907, 1910, HANsey 1908, Wollebek 1908, Stephexsex 1913). Sie ist etwas öfter als die vorige Art in Wasser von niedrig positiver Temperatur gefunden worden (angeblich so$\left(\mathrm{gar}+2^{\circ}\right)$, ist aber ihrer ganzen Verbreitung nach eine ebenso unzweifelhaft hocharktische Art.

\section{Panarktische Arten.}

Als panarktisch bezeichne ich Tiere, welche in der ganzen arktischen Region, meist auch - wie die hiehergehörigen Decapoden - in den boreoarktischen Gebieten vorkommen. Sie gedeihen sowohl in Wasser yon negativer wie von niedrig positiver Temperatur und kömnen von diesem Gesichtspunkte aus arktisch-eurytherm genannt werden.

$\mathrm{E}$ is f jorda $\mathrm{r}$ t e $\mathrm{n}$. Sabinea septemcarinata kamn als eine typische panarktische Art bezeichnet werden. Sie ist allgemein in allen arktischen und boreoarktischen, fehlt aber in den borealen Gebieten; höchstens tritt sie selten in der unmittelbaren Nähe der boreoarktischen Gegenden auf.

Sclerocrangon boreas hat an der norwegischen Küste eine ähnliche Verbreitung wie S. seplemcarinala und fehlt an der ganzen Kiiste siidlich von Helgeland, unterscheidet sich aber ron ihr in wichtigen Hinsichten. Erstens ist er im nordwestlichen Norwegen nicht auf die boreoarktischen Fjorde beschränkt, sondern kommt auch im warmen Gebiet ror. Noch wichtiger ist das Vorkommen bei den Väröern, an der borealen Küiste von Island und an der Westkiiste von Nordamerika südlich vom Beringsmeer.

Es lässt sich nicht leugnen, dass diese Verbreitung einige gegenwärtig schwer vercinbare Widerspríche aufweist. Bei den Färöern ist die Art nach HANSEN (1908) allgemein und lebt in mehr oder weniger seichtem Wasser (wenige bis $180 \mathrm{~m}$ ), also unter rein borealen Bedingungen (einige Fundorte liegen an der offenen Küste); nach der Verbreitung an der skandinavischen Küste muss das Vorkommen hier und an der Westküste von Island (wovon leider keine näheren Angaben vorliegen) ziemlich ïberraschend erscheinen. Entweder ist $S$. boreas eigentlich arktisch-boreal, obgleich an spezielle I'emperaturbedingungen gebunden und aus unbekannten Ursachen südlich von den Lofoten fehlend, oder er lebt bei den Färöern (und in den übrigen warmen Gegenden) hauptsächlich an Lokalitäten mit ganz besonderen Bedingungen. Unter diesen Umständen könnte man natïrlich diese Art zu den arktisch-borealen Arten rechnen; solange die Lebensbedingungen an den borealen Fundorten nicht genau untersucht sind, ist es jedoch berechtigt, das Hauptgewicht auf die Verbreitung an der skandinavischen Küiste zu legen; anch die iibrige boreale Verbreitung ist ja so beschränkt, dass die Art sich scharf von den unzweifelhaften arktisch-borealen Decapoden unterscheidet. Jedenfalls ist $S$. boreas cine verhältnismässig eurytherme Art, die mehr als nur voriibergehend eine Temperatur von wenigstens $+5^{\circ}$ erträgt. Mit dieser Annahme stehen die Verhältnisse im Eisfjord, wo diese Art im warmen Oberflächenwasser ziemlich allgemein ist, in gutem Einklang,

Spirontocaris turgida und S. spinus haben in dem Übergangsgebiet zwischen der horroarlitischen und der horealen Region ungefähr dieselbe Terbreitung wie soleroctungon 
boreas - obgleich die letztere Art vielleicht etwas häufiger im borealen Lofotengebie ist -, kommen aber ausserdem selten weiter suidlich vor, S. turgide nur an ganz vereinzelten Stellen (Trondhjemsfjord, Kristianssund, ein einziger Fund an der schwedisehen Küste), S. spimus etwas häufiger (Trondhjemsfjord, Hardangerfjord, mehrere Funde im Skagerak, vereinzelte in Kattegatt und Öresund; ferner Schottland, Färöer, Westisland).

S. turgida kommt so äusserst selten, grösstenteils wohl nur ganz zufällig in borealen Gegenden vor, dass man sie ohne jede Frage mit den rein panarktischen Tieren vereinigen muss. S. spinus könnte wohl mit gleichem Recht arktisch-boreal genannt werden, ich führe sie aber in diesem Zusammenhang auf, weil sie sich in natürlicher Weise S. turgida anschliesst; wie man eine Art benennt, bleibt doch schliesslich eine rein formelle Frage, die Hauptsache ist, ein möglichst tiefes Verständnis der Verbreitung und der Verbreitungsbedingungen zu gewinnen. Es ist iberhaupt ungewiss, ob die beschränktere Verbreitung von S. turgida auf einem Unterschied in der Thermopathie beruht. Diese Art ist eine ausgeprägte Seichtwasserform und also in siddlichen Gegenden grösseren Temperaturschwankungen als $S$. spimus ausgesetzt, welche sich dort in etwas grössere Tiefe zurichziehen kann. Damit ist keineswegs gesagt, dass ein Unterschied in der Thermopathie ausgeschlossen ist; $S$. turgida scheint unter arktischen Bedingungen ein Sommermaximum von mehr als $+3^{\circ}$ zu meiden; von $S$. spinus kann in dieser Hinsicht nichts Bestimmtes gesagt werden (s. den Speziellen Teil, S. 7, 14).

Sabinea septemcarinata und Spirontocaris spinus nehmen dadurch eine Sonderstellung unter den arktischen Decapoden ein, dass sie sehr nahe Verwandte in der borealen Region besitzen, Subinea sarsii (SMITI) und Spirontocaris lilljeborgii (DANIELSSEN). ApPellöF (1906) hat den Nachweis erbracht, dass auf der europäischen Seite des Nordmeeres keine Übergänge zwischen der arktischen und der borealen Form vorhanden sind, weshalb sie als getrennte Arten betrachtet werden miissen. Seiner Ansicht, dass die Spaltung im Anschluss an die Eiszeit, ngleichzeitig mit einer Veränderung der physikalischen Verhältnisse des Meeres» vor sich gegangen sei, ist durchaus beizustimmen (weniger wahrscheinlich ist, dass sie so spät wie in spät- oder postglazialer Zeit erfolgt ist). Dagegen muss man es wohl vorläufig unentschieden lassen, ob die boreale Form, wie ApPELLÖF annimmt, aus der arktischen entstanden ist; es fehlen noch alle sicheren Anhaltspunkte zur Beurteilung dieser Frage, da man nicht eimmal sicher behaupten darf, dass die morphologischen Unterschiede erblich fixiert sind.

Ü b r i g e A r te n. Ausser den im Eisfjord lebenden Arten gibt es im Nordmeer mit Sicherheit nur zwei panarktische Decapoden, nämlich śpirontocaris groenlandica (FABR.) und Nectocrangon lar OwEN. Beide sind westliche Arten, im Nordmeer nur an der Ostküste von Grönland lebend; ein genauerer Vergleich mit den iibrigen Nordmeerarten ist daher nicht möglich (s. ApPELlöF 1906, HANSEN 1908, STEPHeNSEN 1913).

Im Vorübergehen kann auch Bythocaris simplicirostis G. O. SARs hier genannt werden. Wenn diese Art, wie HANSEN glaubt, mit B. panschii BucHoLz identisch ist. so scheint sie hauptsächlich arktisch zu sein; wenn die beiden formen getrennt sind, so würde die erste niederarktisch-nördlichboreal, die letztere, nach den bisherigen Funden zu urteilen, arktisch, sogar hocharktisch, sein. Doch ist sowohl die Systematik wie die Verbreitung dieser Formen ganz ungeniigend bekannt kgl. Haxsex 1908, Appetzök 1906). 


\section{If. Irktisch-horeale Arten.}

Die arktisch-borealen Decapoden muissen in zwei Gruppen eingeteilt werden. Diese unterscheiden sich, wie APPELLöF hervorhebt, durch ihre verschiedene Verbreitung in der arktischen Region; dazu kommen meiner Auffassung nach tiefgehende Unterschiede nicht nur in der gesamten Verbreitung und Ókologie, sondern auch in der Herkunft.

1. Panarktisch-boreale Arten.

Spirontocaris polaris und $S$. gaimardii sind allgemein in der ganzen arktischen und einem grossen Teil der borealen Region. Die erstere Art ist im Nordmeer suidlich bis in die Fjorde der schwedischen Skagerakküste sowie bis an die Shetlandinseln, Hebriden und Färöer verbreitet: an der skandinavischen Kuiste ist sie demnach nicht einmal so weit südwärts wie $S$. spim bekannt, im Gegensatz zu dieser ist sie aber in ihrem borealen Verbreitungsgebiet häufig und iberall regelmässig vorhanden. S. gaimardii dringt weiter nach Süden; sie lebt noch im Öresund, in der suidwestlichen Ostsee und an der Ostund Westkiiste von Schottland.

Die Herkunftron Spirontocaris polaris ind gaimardii.

Eine Analyse der Verbreitung und Lebensverhältnisse der beiden panarktisch-borealen Decapoden gibt das Resultat, dass sie sich den borealen Bedingungen sekundiir angepasst haben; sie können als ursprïnglich arktisch betrachtet werden. Sie schliessẹ sich demmach den oben erwähnten arktischen Arten eng an, obgleich sie nach ihrer tatsächlichen Verbreitung als arktisch-boreal bezeichnet werden müssen. In meiner Arbeit iiber die Echinodermen des Eisfjords habe ich die Voraussetzungen und die 'Tragweite einer solchen Annahme arktischer Herkunft von arktisch-borealen Tieren untersucht (S. 232-242); hier will ich nur die Decapoden betrachten.

Besonders einfach und klar ist, wie APPELLöF gezeigt hat, diese Schlussfolgerung in bezug auf $S$. polaris. Der erwähnte Forseher legt das Hauptgewicht auf die beschränkte Vertikalverbreitung in der borealen Region; die Art lebt dort in der Regel exst in einer Tiefe von mehr als $100 \mathrm{~m}$, also in Wasserschichten von verhältnismässig niedriger und konstanter Temperatur $\left(+5-+7^{\circ}\right)$. Diese Tatsache beweist jedoch mit völliger Sicherheit nur, dass die Art eine höhere und stark wechselnde Temperatur nicht erträgt, nicht aber, dass sie arktische Bedingungen bevorzugt. Tch habe oben nachzuweisen versucht, dass sie in arktischen Gegenden in der Regel nur in Wasser vorkommt, dessen Temperatur nie über $+3^{\circ}$ steigt; wenn ihr solches Wasser zu Gebote steht, meidet sie also nicht nur Wasser von +5 bis $+7^{\circ}$ konstanter Temperatur, sondern sogar eine voribergehende Erwärmung des Wassers. Hierzu kommt der Umstand, dass die Art unter borealen Bedingungen auch anuähernd nicht dieselbe Körpergrösse wie in arktischen Gegenden erreicht (s. Appeltör 1906, S. 121; nähere Angaben ïber die Grösse borealer Individuen fehlen leider).

Das Vorkommen in borealen Gegenden ist also erst durch ein sekundäres Gewöhnen, eine "Anpassung» an Temperaturverhältnisse, welche das Tier unter ursprünglichen Bedingungen meidet, ermöglicht worden. ApPELLöF spricht von einer Anpassung in der vertikalen Verbreitung; dabei ist jedoch keine Anpassung an neue Verhältnisse eingetreten, denn das Tier lebt ja auch an den arktischen Kuisten in der Tiefe, und der Unter- 
schied besteht nur darin, dass es bei wärmerem Oberflächenwasser ausschliesslich dort vorkommt.

Wenn diese Auseinandersetzungen richtig sind, zeigt S. poluris unter urspruinglichen Bedingungen ziemlich genau dieselbe Thermopathie wie die fast rein arktische S. turgida und wie S. spinus. Der Unterschied diesen gegeniiber besteht also lediglich in einem grossen Anpassungsvermögen. Auch S. spinus tritt ja indessen in der borealen Region auf und zwar an ähnlichen Lokalitäten, obgleich mvergleichlich seltener als S. polaris. Dass es sich auch hierbei um eine sekundäre Gewöhnung an die borealen Bedingungen handelt, liegt offen zu 'Tage; das Anpassungsvermögen dieser Art muss viel schwächer als bei $S$. polaris sein.

S. gaimardii lebt in der borealen Region in ganz seichtem Wasser und zwar nur dort, obgleich sie an arktischen Küsten in viel grösserer Tiefe allgemein ist. Diese Art und S. polaris haben also unter borealen Bedingungen ganz entgegengesetzte Besehränkungen in ihrer vertikalen Verbreitung erfahren; diese fehlt im oberen, jene im unteren Teil ihres ursprünglichen Verbreitungsgebiets. Ich habe oben (S.34) die lichtvolle Hypothese erwähnt, mit der APPELLöF die eigentïmliche Vertikalverbreitung von S. gaimardii erklärt; sie sei, um das Gesagte kurz zu wiederholen, nur für ihre Lutwicklung von kaltem Wasser abhängig und finde daher gute Existenzbedingungen in der im Winter kalten Uferzone der borealen Gegenden. Eine andere Erklärung dürfte in der Tat nicht denkbar sein. Wenn die Art also in ihrer Fortpflanzung ausgesprochen stenotherm, ja sozusagen arktisch ist, so folgt daraus noch nicht, dass sie urspringlich nur unter arktischen Bedingungen gelebt hat. Doch erhält diese Hypothese hierdurch eine grosse Wahrscheinlichkeit, besonders weil die Tortpflanzung oder vielleicht eher Entwicklung sogar fast rein arktische Temperaturen zu erfordern scheint oder das Tier wenigstens unter solchen am besten gedeiht. Hierzu kommen die Tatsachen, dass die Art noch viel allgemeiner in den arktischen Gebieten ist als in irgend einem borealen und eine bedeutendere Körpergrösse erreicht (s. Appeluöf, Birula 1910 u. a.); die boreale Rasse macht ferner morphologisch einen reduzierten Eindruck. Unter diesen Umständen muss man die Нypothese eines arktischen Ursprunges als wohl begrüdet ansehen. Dagegen kamn man nicht entscheiden, ob diese Art ursprünglich ebenso stenotherm wie z. B. S. polaris gewesen ist (s. näheres im Speziellen Teil, S. 35). Wenn sie in den kältesten Gebieten in der Uferzone, wo sie im Eisfjord selten ist, allgemein rorkommt, ist dies offenbar der Fall. Dann hat sie in der borealen Region ihre Vertikalverbreitung nur eingeschränkt; wenn sie aber in allen arktischen Gegenden in seichtem Wasser sehr selten ist, hat sie, im Gegensatz zu S. polaris, ihre bathymetrische Verbreitung wirklich verändert. Was die Fortpflanzung betrifft, darf man nicht einfach sagen, dass die Art unter borealen Bedingungen ihre Fortpflanzungszeit verändert oder in den Winter "verlegt» hat, da wenigstens im Eisfjord die Embryonalentwicklung ebenfalls erst im Winter vorsichzugehen scheint; dagegen scheint (nach den Verhältnissen in Nordnorwegen zu urteilen) die Zeit der Eiablegung verschieden zu sein (s. oben S. 35).

\section{Niederarktisch-boreale Arten.}

Zu dieser Gruppe gehören Pandalus boreatis, Eupagums pubescens und Hyas aruneus. APPELLöF hat darauf aufmerksam gemacht, dass diese Arten man mehreren ty- 
pisch hocharktischen Kiisten» fehlen. Wine genaue Untersuchung der Verbreitung lehrt zwar, dass sie nicht rollständig in allen hocharktischen Gegenden fehlen — so leben sie selten oder sogar ( $E$. pubescens) etwas häufiger in Ostspitzbergen, die letztere Art ist einmal in Nordostgrönland gefunden usw. - gleichzeitig wird es aber noch klarer als zuvor, dass die Verbreitung ganz überwiegend niederarktisch ist und dass dabei die Temperaturverhältnisse ausschlaggebend sind.

In ihrer borealen Verbreitung repräsentieren diese Arten zwei rersehiedene 'Typen. $P$. boreatis hat eine beschränkte Verbreitung in der borealen liegion; an der skandinavischen Küste lebt er regelmässig und in Menge bis in den nördlichen Teil des Kattegatt, selten siidlich davon bis in den Oresund: an den westlichen britisehen Küsten fehlt er vollkommen, und auch im nördlichen T'eil der westlichen Nordsee scheint er nur als grosse Seltenheit aufzutreten. E. pubescens und II. araneus zeigen in ihrer Verbreitung grosse Ahnlichkeiten, wenngleich die erstere Art an den europäischen Küsten mehr nördlich ist; sie lebt bis in den sidlichen Teil des Kattegatt (dort jedoch nicht allgemein), in der nördlichen Hälfte der westlichen Nordsee, an der Westkiiste von Schottland und im benachbarten 'T'eil der Irischen See, endlich südwestlich von Irland. Hyas araneus ist dureh die ganze Nordsee und den Kanal verbreitet und lebt an allen britischen Kiisten. Der wichtigste gemeinsame ' Zug in der Verbreitung dieser Arten ist, dass sie den Färö-Shetland-Riicken überschreiten und ausserhalb der Grenzen des Nordmeeres leben.

Pandalus borealis ist ein lehrreiches Beispiel einer Art, deren Verbreitung dureh ganz bestimmte Anforderungen an gewisse äussere Bedingungen geregelt wird. Die Verbreitung ist in sehr verschiedener Weise gedentet worden; es scheint mir von Interesse, die ïber dieses viel umschriebene Tier geäusserten Ansichten kurz zusammenzustellen.

Pandalus borealis wurde lange als ein echt arktisches 'T'ier aufgefasst, im Süden nur als Relikt lebend (M. SARS, G. O. SARS). Diese Ansicht fusst anf dem erst vor wenigen Jahren widerlegten Glauben, dass die Art an der skandinavischen Kuiste nur in vereinzelten kalten Fjorden zu finden sei, und war daher ganz naturlich; wie aber DoFters (1900) zu der Ansicht gelangen komnte, dass sie in arktischen Gegenden rorwiegend im kältesten Wasser zu finden sei, ist schwer verständlich. OHLIN (1901) war der erste, der auf das Fehlen in den kältesten arktischen Gegenden anfmerksam machte; die Art sei daher nicht als eine echt arktische Form, sondern mather as an North Atlantic (and North Pacific) species》 zu betrachten. Gegen diese Ansicht wendet sich Appeluör (1906); die Art ist nach ihm arktisch, „weil sie auch im Wasser negativer Temperatur normalerweise auftreten" kam, und darf nicht nordatlantisch genannt werden, denn sie fehlt im atlantischen Meere ausserhalb des Nordmeeres (tatsächlich dürfte der Gegensatz. zwischen diesen Auffassungen ziemlich geringfügig sein; APPELLÖF versteht hier unter "arktisch" dasselbe wie arktisch-boreal, OmLin unter "North Atlantic» eben das Nordmeer). Nach Hansen (1908) ist die Art iscarcely so marked an aretic form as various other decapods"; die Ansicht OHLIns sei jedoch "somewhat exaggerated". WOLLEBAK (1908) und BJörck (1911), sowie Ekman, Pettersson \& 'Tríbom (1910) betonen, dass die Art der skandinavischen Küste entlang häufig und an gewisse Wassersehichten gebunden ist; die frithere Auffassung, sie sei ein glaziales Relikt, ist daher unrichtig. NoRMAx 
(1909) bezeichnet $P$. borealis schlechthin als ma truly aretic species». STEPHENSEN nannt ihn vor einigen Jahren (1910) eine "wesentlich arktische" Art, rechnet ihn aber numehr $(\mathbf{1 9 1 2}, 1913)$ zur matlantischen (borealen) Tiefseefauna », also zu einer Gruppe, deren Nordgrenze in der Regel auf dem Rïcken Shetlandinseln-Färöer-Island-Grönland-Balffin-Tand liegt. Grieg (1914) rechnet die Art zu einer Gruppe von friher als Relikte aufgefassten Formen, die sich als »boreal oder von noch suidlicherem Ursprung erwiesen haben».

Nach den gegenwärtig bekannten Tatsachen der Verbreitung darf man $P$. boreulis ebensowenig als arktisch wie als rein boreal bezeichnen. Am allerwenigsten gehört er zu Stephensens atlantischen Tiefseearten; im östlichen Teil des Nordmeeres stellt ja die von diesem Autor für die Gruppe angegebene Nordgrenze eben die Südgrenze dar, und das Tier lebt ïberhaupt nie in rein atlantischem Wasser (eine Tiefseeart ist es auch nicht; die Angabe Stephensens, dass es meist viel tiefer als $180 \mathrm{~m}$ lebt, ist unrichtig). Mit etwas grösserer Berechtigung könnte man die Art "vorwiegend boreal» nennen, da sie in arktischen Gegenden fast nur in mit Golfstromwasser gemischtem Wasser vorkommt. Doch ist sie dort allgemein und lebt in Gesellschaft von zahlreichen rein arktischen Tieren, weshalb sie richtiger zu der hier unterschiedenen niederarktisch-borealen Gruppe zu zählen ist.

Wichtiger als die Namensfrage ist es, die Tatsache zu betonen, dass die Art von verhältnismässig wenig wechselnden hydrographischen Bedingungen abhängig ist. Vor allem gilt dies von der Wassertemperatur. APPELLör findet es zwar möglich, aber nicht erwiesen, dass sie niberwiegend in Wasser positiver Temperatur" lebt, und betont, dass sie "normalerweise» in Wasser von negativer Temperatur auftritt; nummehr kann es jedoch als festgestellt gelten, dass sie Wasser von konstant negativer Temperatur meidet. Natiurlich kann man nicht behaupten, dass die untere Temperaturgrenze gerade bei $0^{\circ}$ liegt; in Wasser von $0-+2$ Temperatur wird die Art häufig gefunden, es ist aber wohl trotzdem nicht ausgeschlossen, dass sie sich vorzugsweise in etwas wärmerem Wasser aufhält oder fortpflanzt. Von etwa $+2^{\circ}$ an gedeiht sie jedenfalls ebenso gut wie in borealen Gegenden; dies geht sowoht aus der allgemeinen Verbreitung wie besonders aus dem häufigen Torkommen in der Eisfordtiefe hervor (man könnte den Findruck bekonmen, dass sie im borealen Gebiet in grösserer Menge auftritt; die anscheinend grössere Häufigkeit dürfte jedoch darauf beruhen, dass nur dort eine wirkliche Fischerei betrieben wird), Sie ist also keineswegs, wie man nach einigen Äusserungen der neueren skandinavischen Autoren vermuten könnte, an die hohe Temperatur gebunden, unter welcher sie an der skandinavischen Küste lebt. Hier trifft man sie in der Regel in Wasser von +5 bis +6 $\left(+4\right.$ bis $\left.+7^{\circ}\right)$ Temperatur (und 34 bis $35 \%$ Salzgehalt) (WoLleBek, BJörck). Gelegentlich kann man sie in noch wärmerem Wasser finden (BJ ÖRCK: 2 Fundorte + 8,5.5 bzw. 11,30 ${ }^{\circ}$, doch muss in solchen Fällen entweder das Auftreten des Tieres oder des warmen Wassers zufällig sein; die obere Temperaturgrenze für das regelmässige Vorkommen kann nicht viel oberhalb von $+6^{\circ}$, kaum oberhalb von $+7^{\circ}$ liegen. - Ủber die Abhängigkeit vom Salzgehalt s. oben S. 73.

Auch die Verbreitung von Eupagums pubescens und Hyas araneus kann mit einer Abhängigkeit von gewissen Temperaturverhältnissen in Zusammenhang sebrachi werden. Die untere Temperaturgrenze ist ungefähr dieselbe wie fuir Pundulus boreatis (nach 
cinzelnen Befunden ertragen sie möglicherweise etwas besser kaltes Wasser, nach anderen nicht), die obere liegt dagegen bedeutend höher. E. pubescens erträgt an der skandinavischen Küste, der vertikalen Verbreitung nach zu urteilen, ein Sommermaximum von

$12^{\circ}$ odler etwas mehr (Jahresamplitude z. B. $+6-+12^{\circ}$ ); an der Ostkiuste von Nordamerika lebt er in Wasser von konstant ziemlich hoher 'T'emperatur (Sмттн [1884 b, 1887] verzeichnet 42 Fundorte mit +6 bis $+12,2^{\circ}$ Temperatur, dazu ein Fundort mit $+17,2^{\circ}$ T'emperatur). H. araneus ist noch eurythermer; nach der Verbreitung im Englischen Kanal usw. muss er eine Jahresamplitude von +7 oder sogar $9-+16$ oder $17^{\circ}$ ertragen. An der Ostkuiste von Amerika geht auffallenderweise die weniger eurytherme, an den curopäischen Küsten mehr nördliche Art E. pubescens bedeutend weiter südlich als die bei uns weiter suichärts verbreitete Art H. araneus. Dieser Gegensatz hängt wohl wenigstens teilweise mit der verschiedenen bathymetrisehen Verbreitung zusammen (doch ist es wohl nicht ausgeschlossen, dass $H$. araneus nicht mbedingt eurythermer ist als $E$. mbescens; es ist z. B. denkbar, dass die erstere Art ein hohes Maximum besser, aber eine andauernd sehr hohe 'Temperatur weniger gut erträgt).

Die drei niederarktisch-borealen Deca poden sind im Vergleich mit den arktischen Arten Warmwassertiere, unterscheiden sich aber nicht mwesentlich voneinander. Eupagurus pubescens und Hyas araneus sind verhältnismässig eurytherm; Pandalus boreatis ist ziemlich ausgesprochen stenotherm, ungefähr ebenso stenotherm wie die arktisch-eurythermen, wohl mehr stenotherm als manche boreale Arten. Wegen der Ähnlichkeiten in der Verbreitung — welche mit der Vertikalverbreitung zusammenhängen; eine Litoralart mit der Thermopathie von $P$. borealis würde eine äusserst beschränkte Verbreitung haben und vor allem wegen der ähnlichen unteren 'Temperaturgrenze kann man sie trotzdem zu einer gemeinsamen Gruppe vereinigen.

Die Herkunft ron Pandalus borealis, Eupagurus pubescens un d Hyas araneus.

Über die Herkunft dieser drei Arten hat sich - abgesehen von den älteren Autoren, denen die weite Verbreitung in der borealen Region nicht bekannt war - hauptsächlich Appelü̈ (1906) geåussert. Von andern neueren Autoren ist eigentlich nur Birula (1910) zu nemnen; er bezeichnet Hyas araneus kurz als neine Art borealer Herkunft", ohne jedoch auf die Verbreitung oder Verbreitungsgesehichte einzugehen. Was Pandalus borealis betrifft, scheinen, nach den oben angefuihrten Angaben zu urteilen, STephensen und GRIEq einer ähnlichen Ansicht zu sein.

Nach ApPeLLöF dagegen haben diese Arten, wie Spirontocaris polaris und S. gaimardii, einen arktischen Ursprung; sie fehlen zwar in den kältesten arktischen Gegenden, dass sie aber min jedem Falle unter arktischen, wenn auch nicht hocharktischen, physikalischen Verhältnissen entstanden sind, darauf deutet der Umstand, dass sie in der arktischen Zone ihre kräftigste Entwicklung erreichen».

Diese Ansicht ist in der allgemeinen Auffassung AppezLörs begrüdet, dass alle arktisch-borealen Tiere ursprïnglich arktisch sind und sich erst sekundär dem Leben unter borealen Bedingungen angepasst haben. Ich habe in meiner Arbeit iiber die Echinodermen des Eisfjords diese Hypothese diskutiert und bin dabei zu dem Ergebnis gekommen, dass diese allgemeine Voraussetzung fehlerhaft ist. Man muss in jeden Falle 
prüfen, ob einige Anzeichen einer arktischen Herkunft vorhanden sind; wem dies nicht der Fall ist, hat man nicht das Recht, eine solche vorauszusetzen, sondern muss von dem folgenden Grundsatze ausgehen: wenn eine Art iiber zwei Gegenden mit verschiedenen äusseren Bedingungen verbreitet ist und in beiden ebenso gut gedeiht (ebenso allgemein ist, dieselbe Körpergrösse erreicht usw.), muss man sich rorstellen, dass sie von Anfang an den Bedingungen beider Gebiete angepasst gewesen ist.

Ich werde num nachprüfen, ob die drei fraglichen Arten einige spezielle Zeichen einer arktischen Herkunft aufweisen; nach APPELLÖF wïrden sie ja ihre bräftigste Entwicklung" unter arktischen Bedingungen erreichen.

Pandalus borealis ist ohne den geringsten Zweifel ganz ebenso allgemein im borealen wie im arktischen Teil seines Verbreitungsgebietes. Über die Körpergrösse liegen folgende Angaben vor. Arktische Gegenden: Hansen 1887: Maximallänge $129 \mathrm{~mm}$; OHLIN 1901: "One of the largest specimens》 $122 \mathrm{~mm}$; Birula 1907: Maximallänge 125 mm; Stephensen 1912 a: Naximallänge $155 \mathrm{~mm}$; Eisfjord nach dem von uns und der Expedition 1900 gesammelten Material: Maximallänge etwa $145 \mathrm{~mm}$. Boreale Gegenden: WolLEB Ek 1908: Westkïste von Norwegen und Skagerak: keine Angabe der Maximallänge, die meisten eiertragenden Weibchen 120-160 mm; Pettersson, Ekmax \& TryBoм 1910, BJöRck 1911, Skagerak: Maximallänge (der Weibchen) $185 \mathrm{~mm}$, Exemplare von $160 \mathrm{~mm}$ Länge nicht selten. Diese Angaben beweisen nicht, dass die Art im borealen Gebiet grösser wird (weil sie dort in viel grösserem Massstabe gefangen worden ist), wohl aber, dass sie dort wenigstens ebenso gross ist wie unter arktischen Bedingungen.

Hyas araneus ist in der ganzen borealen Region des Nordmeeres allgemein oder wenigstens nicht selten (an der schwedischen Westküste müberall häufig», s. TnÉEL 1907, LAGERBERG 1908). In arktischen Gegenden ist er zweifellos nicht häufiger. BIRULA (1907) meint sogar, dass das eigentliche Verbreitungsgebiet, vd. h. das Areal des massenhaften Vorkommens» sich im borealen Küstengebiet befindet. Diese Ansicht dürfte jedoch etwas iibertrieben sein; sowohl unsere wie frühere Beobachtungen zeigen, dass die Art in Wasser von niedrig positiver 'Temperatur ziemlich häufig sein kann; wegen ihrer Lebensweise erhält man sie in keiner Gegend in grösserer Menge. Ủber die Körpergrösse liegen nur wenige Angaben ror, sie scheint jedoch in beiden Gebieten ungefähr dieselbe zu sein; ich nenne einige Angaben über die Maximallänge des Carapax. Arktische und boreoarktische Gegenden: HANSEN 1887 (»ein riesenhaftes Mänuchen»): 107 mm; 1908: $110 \mathrm{~mm}$; Brrula 1907 («ein ziemlich grosses Exemplar»): $74 \mathrm{~mm}$; Wisfjord: $88 \mathrm{~mm}$. Westfinmmarken: Dons 1913: $112 \mathrm{~mm}$. Boreale Gegenden: LAGERERG 1908, Westkiiste von Schweden: 104,3 mm; BeLL 1853, britische Kiisten: etwa $89 \mathrm{~mm}$.

Eupagurus pubescens ist im Skagerak und Kattegatt nicht sehr selten, jedoch zweifellos weniger allgemein als in niederarktischen und boreoarktischen Gegenden (vgl. IAGERBERg 1908, StEPHENSEN 1910). Daraus folgt jedoch nicht, dass boreale Bedingungen ihm weniger gut als arktische zusagen, denn an der norwegischen Kuiste ist er nach Al' PELLöF (1906) )ausserordentlich häufig und allgemein verbreitet». Auch in andern warmen Gegenden scheint er allgemein zu sein, wenigstens an der Ostkiiste von England (Norman \& Brady 1911) und an der amerikanischen Küste siidlich ron K. ('od. Angaben über die Körpergrösse sind spärlich vorhanden und gestatten keinen näheren Ver- 
gleich (maximale Carapaxlänge in Westspitzbergen nach OmLn [1901] 25 mm, nach unseren Beobachtungen $28 \mathrm{~mm}$; an der schwedischen Küste nach LAGERBERG [1908] $23 \mathrm{~mm}$ ). Aurivillius (1886) beobachtete in der boreoarktischen Region Norwegens (Krvaenangsford) meist nur kleine Exemplare (vgl. auch Doss 1915) und findet sogar, dass die Art dort im allgemeinen nicht dieselbe Entwicklung wie an suidlicheren Kiisten zul erreichen scheint.

Die drei Arten - ïber $E$. pubescens muss man sich vielleicht, besonders was die Körpergrösse betrifft, mit einigem Vorgehalt äussern — sind also ebenso allgemein und kräftig entwickelt in borealen wie in arktischen Gegenden. Andere Umstände, welche auf einen arktischen Ursprung hinweisen, sind nicht bekannt. Eine 'Tatsache könnte jedoch in dieser Weise gedentet werden und darf daher nicht versehwiegen werden: die Himbryonalentwicklung geschieht wenigstens teilweise im Winter.

Am besten bekannt sind die Fortpflanzungsverhältnisse von Pandalus borealis. Wie. WoLlEB EK (1908) für das siidliche Norwegen, BJöcRK (1911) für die schwedische Küiste nachgewiesen haben, laicht diese Art dort im Sommer; die Eier werden in der Regel im September abgesetzt, ihre Entwicklung findet im Herbst und Winter statt, und die Larven schliipfen im Februar oder März aus.

Tch habe in meiner Echinodermenarbeit (S.236 ff.) die Bedeutung der Fortpflanzungs- und Entwicklungszeit für die Frage der Herkunft erörtert; der Umstand, dass eine Art sich in borealen Gegenden in der kalten Jahreszeit fortpflanzt oder entwickelt, ist meiner Ansicht nach kein hinreichender Beweis fuir die Ammahme, dass sie urspriinglich rein arktisch gewesen ist. In diesem Falle beweist die Entwicklungszeit noch weniger als sonst, denn man kann nicht behaupten, dass die Entwicklung kälteres Wasser erfordert als die iibrigen Lebensfunktionen. Die neuen Untersuchungen iiber P. borealis (WOLLEBæK, Bлö̈RK) haben das Erbgebnis geliefert, dass die Art in der borealen Region stets, also anch wïhrend der Embryonalentwicklung, in Wasser desselben Ursprungs und ungefähr derselben Beschaffenheit lebt; im Anfang des Jahres sucht sie in den Fjorden seichteres Wasser auf, weil das Bodenwasser damn gegen die Oberfläche steigt. Die Larven der frïheren Stadien leben wohl teilweise in etwas kälterem Wasser, zum grossen, vielleicht grössten Teil jedoch auch in tieferen Schichten (s. BJöRck).

Ferner darf man nicht vergessen, dass auch verschiedene südliche 'Tiere, deren Embryonalentwicklung erwiesenermassen dureh Wärme (natiorlich innerhalb gewisser Grenzen) gefördert wird, sich teilweise im Winter fortpflanzen. Crangon crangon scheint zweimal im Jahre zu laichen; die Embryonalentwicklung nimmt im Winter ebensoviele Monate in Anspruch wie im Sommer Wochen (EHREnbaum, zitiert nach WoLleb ex 1908). Der Hummer trägt in Norwegen seine Eier ein ganzes Jahr, im Winter tritt aber cin fast völliger Stillstand in der Entwicklung ein; in siudlicheren Gegenden mit wïmerem Wasser ist die 'Tragzeit kürzer (AppeltöF 1909). Nach einigen Beobachtungen ist es num nicht unwahrscheinlich, dass $P$. borealis sich ähnlich verhält; in den Fjorden des nördlichen Norwegens scheint nämlich nach den Angaben WoLLEBæK's die Entwicklung längere Zeit in Anspruch zu nehmen als in suidlicheren Gegenden (Ausschliupfungszeit April statt Februar oder März). Zuletzt ist zu bemerken, dass die boreale Art Ponlophilus norvegicus fast ganz dieselbe Fortpflanzungszeit hat wie P. borealis (WoLIEBak). 
Über die Fortpflanzung und Entwicklung der beiden iibrigen Arten ist wenig bekannt. APPELLöf (1912) rechnet zwar Hyas araneus zu denjenigen arktisehen "Reliktformen », bei denen die Fortpflanzung in die kälteste Jahreszeit fällt; aus den angeführten Beobachtungen - er hat neu ausgeschliipfte Larven im Januar und Februar beobachtet, ausserdem ist vielleicht auch eine zweite Entwieklungsperiode im Frihling vorhanden _ folgt aber nicht, dass die Entwicklung ausschliesslich in der kalten. Jahreszeit vorsichgeht. Die Beobachtungen von Auriviluius (1898) an der schwedischen Küste scheinen zu erweisen, dass keine strenge Periodizität vorhanden ist; dieser Forscher fand Eier in verschiedenen Entwicklungsstadien von Februar bis Ende September (vgl. auch DALYELL 1851). In einer nördlichen Gegend (Westfimmarken) hat neuerdings Dons (1913) ähnliche Beobachtungen gemacht. Die Embryonalentwicklung von Eupagums pubescens geschieht im nördlichen Norwegen im Winter (WoLLEB de 1905, Dons 1915). Wahrscheinlich liegen die Verhältnisse ähnlich weiter südlich, vielleicht aber auch in arktischen Gegenden (vgl, oben S. 60).

Das Resultat der obigen Erörterungen ist, dass weder in der Verbreitung noch in der Ökologie dieser drei Arten einige Züge bekannt geworden sind, welche eine sekundäre Anpassung an den borealen Bedingungen wahrscheinlich machen. Man muss sich demnach wenigstens bis auf weiteres vorstellen, dass die hentige Thermopathie urspruinglich ist. In bezug auf die bestbekannte Art, Pandalus boreatis, spricht nicht nur nichts gegen, sondern viele Tatsachen direkt für die Richtigkeit dieser Auffassung. Als eine allgemeine Stiitze derselben muss schliesslich der Umstand hervorgehoben werden, dass die drei Arten hocharktische Bedingungen meiden. $P$. borealis ist ja ziemlich stenotherm; er unterscheidet sich von mehreren borealen Arten, die an Temperaturen von z. B. + 3 bis +7 oder $8^{\circ}$ gebunden sind (unter den Decapoden Sabinea sarsii und Pontophitus norvegicus, s. miten), nur dadurch, dass die untere Temperaturgrenze etwas niedriger liegt. Man kann natürlich die Möglichkeit nicht ausschliessen, dass eine solche Art ursprünglich noch stenothermer und gerade an niedrig positive Temperaturen gebunden gewesen sei; solange aber keine Grinde für eine solche Hypothese vorgebracht werden können, muss sie für sehr unwahrscheinlich gehalten werden.

Von einer Vereinigung dieser niederarktisch-borealen Tiere mit Spirontocaris polaris und gaimardii zu einer gemeinsamen tiergeographisch-ökologisehen Gruppe kann unter diesen Umständen keine Rede sein. Die Kluft zwischen ihnen ist im Grunde tiefer als zwischen den erwähnten Spirontocaris-Arten und den rein arktischen Decapoden, vielleicht auch tiefer als zwischen den niederarktisch-borealen und den borealen Arten.

\section{Boreale Gäste in der arkischen Region.}

Einige boreale Arten treten als mehr oder weniger seltene Gäste in arktischen Gegenden auf, natülich nur in der niederarktischen Zone, und hauptsächlich nur in den Grenzgebieten zwischen dieser und der boreoarktischen Ubergangszone. Selbstverständlich handelt es sich nur um solche boreale Tiere, welche häufig in die boreoarktischen Gegenden eindringen. Vom Eisfjord sind keine solchen Arten bekannt (GRIEG erwähnt Spirontocaris pusiola vom Eisfjord, diese Angabe ist aber unrichtig; s. oben S. 28); vom ïbrigen Nordmeer kennt man deren 4 bis 6 . 
Pandalus monlagni LAACH (anmulicomis) und Pandalina brevirostis (RATHKE) (mediterran-boreale Art) sind, wie es scheint, im nördlichen bzw. mittleren 'T'eil des Barentsmeeres gefangen worden (D'URBAN 1880, HoEK 1882); der Fundort für die letztere Art liegt nicht weit von der boreoarktischen Area vor der skandinavischen Kuiste.

[m Spitzbergengebiet sind + boreale Decapoden beobachtet worden: Pontophilus norvegicus II. SARS (SW. vom Siidkap) [OHLw 1901]); Sabinea sersii SMITII (SW. von Pr. Charles Foreland, NW., W. und SW. von Beeren Liland [BIruLA 1907, WoLleb zEK 1908]), ferner nach Breitruss [1903] im östlichen 'Leil des Barentsmeeres); Lithodes maja (L.) (W. von Beeren Filand [HARTLAUB 1900]); Iyas coarctatus LeACII (Nordwestspitzbergen [SARS 1886]). - Die letztere Art wird von APPELLöF zu den arktisch-borealen Tieren gerechnet. Wie aus den obigen (S. 65-66) Ausführungen iber die Hyas-Formen des Barents- und Karischen Meeres hervorgeht, ist sie im Nordmeer typisch boreal; der erwähnte einzige Fund im arktischen Gebiet ist offenbar ziemlich zufällig. In andern Gegenden dringt sie etwas weiter in die arktische Region ein; sie ist allgemein am sudlichen Teil von Westgrönland (viel häufiger als z. B. Lithodes maja und Sabinea sarsii) und lebt am östlichsten 'T'eil der sibirischen Nordkiiste.

In ihrer borealen Verbreitung und Thermopathie zeigen die vier zuletzt besprochenen Arten einige Unterschiede, welche in diesem /usammenhang von Interesse sein können, weil sie die früher angedeutete Ansicht beleuchten, dass sie eigentlich den niederarktisch-borealen Arten verhältnismässig nahe stehen. Sabinea sarsii ist ostatlantisch eine reine Nordmeerform und scheint in Wasser von höchstens $+8^{\circ}$ T'emperatur zu leben; Pontophilus norvegicus lebt anch im atlantischen Neer südlich vom Nordmeer, ist aber ebenfalls stenotherm (Temperaturamplitude in der Regel $+3-+8^{\circ}$ ) (natiirlich ist diese Art nicht arktischer Herkunf, wie G. O. Sars und Ombin vermutet haben). Diese beiden Arten schliessen sich also P'. boreatis an, von welchem sie sich hauptsächlich durch die höhere Lage besonders der unteren Temperaturgrenze unterscheiden. Lithodes maja und IIyas coarclatus sind bis an die westlichen britischen Kiisten verbreitet und bedeutend eurythermer als die vorigen Arten; sie nähem sich also Eupagurus pubescens und Hyas aremeus (vgl. besonders ApPLLö̈ 1906, HANSEN 1908, für Temperaturangaben auch SMITI 1884 b, 1887).

\section{Zusammenfassende Übersicht der nord-südlichen Verbreitung.}

I. Arktische Arten.

1. Incharktischo Arten.

bylhoceris leucopis (hocharktisch-nbyssal).

menyeri (eurybather).

Selcrocrungon ferox

2. P'anarktischo Arten.

Sabinea septemearinata (rein arktisch).

Sheroerungon borens (eurythermer, in gewissen (iegenden auch unter borealen Bedingungen lebend). spirontocuris turgitu (an ganz sereinzelten stellen in der borealen Region). spinus (etwas häufiger in der borealen Region, nähert sich s. polaris).

Spirontocuris groenlendica.

Vectocrangon lar.

(Bythocaris simplicirostrisp). 
11. Panarktisch-boreale Arten, urspriinglich mehr rein arktisch.

spirontocaris poluris.

gaimardii.

(Bythocaris simplicirostris:).

111. Niederarktisch-boreale Arten (Warmwasserarten).

Pandalus borealis (verhältuismässig stenotherm)

$\left.\begin{array}{l}\text { Eupagurus pubescens } \\ \text { IIyas araneus }\end{array}\right\}$ (mehr oder wenigex eurytherm).

IV. Boreale Arten, als Gäste in niederarktischen Gegenden auftretend.

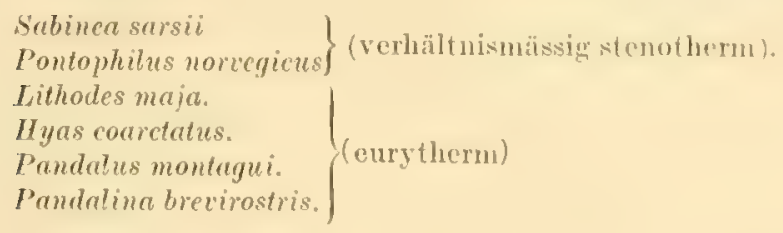

\section{Die ost-westliche Verbreitung.}

In der folgenden kurzen Übersicht beriicksichtige ich alle arktischen und arktischborealen Decapoden (nicht nur wie in der obigen Darstellung die Nordmeerarten), dagegen nicht die borealen Arten. In bezug auf die von mir gebranchte Terminologie verweise ich auf meine Echinodermenarbeit.

\section{Zirkumpolare Arten.}

1. Kontinu ierlich zirkumplare Arten. Spirontocaris turgida, polaris und gaimardii sind von allen arktischen Gegenden bekannt, von welchen mehr als ganz zufällige Beobachtungen vorliegen, und leben im Beringsmeer. Sie sind also mit Sicherheit kontinuierlich zirkumpolar.

2. Nicht vom pazifischen Gebiet bekannt, trotzdem aber wahrscheinlich kontinuierlich zirkumpolar. Sabinea septemcarinata ist nicht im Beringsmeer gefunden, lebt aber an der sibirischen Kiiste unweit davon, und von dort erstreckt sich die Verbreitung ohme Unterbrechung (abgesehen von der tiefen See zwischen Spitzbergen und Grönland) westwärts bis in den nordamerikanisehen Archipel. Rings 11 m das Polarbecken fehlen Fundangaben also nur von einer faunistisch so gut wie umbeliannten Küstenstrecke, und ich halte es daher für wahrscheinlich, dass diese Liicke scheinbar ist. Das Fehlen im Beringsmeer spricht kaum gegen diese Amname. Erstens lebt die Art nahe an der Beringsstrasse; es würde daher auf jeden Fall unerklärt bleiben, warum sie nicht durch dieselbe eindringt. Zweitens ist der nördliche 'Teil des Beringsmeers verhältnismässig ungenügend erforseht; der südliche, besser bekannte 'Ieil dürfte zu warm sein, denn wenn diese Art auch keineswegs hocharktisch ist - in welchen Falle das Fehlen selbstrerständlich wäre - so lebt sie doch nie ausserhalb der arktischen mol boreoarktischen Gebiete.

3. Sclerocrangon boreas und Spirontocaris spinus sind offenbar (die erstere Art mit Sicherheit) ohne Unterbrechung vom Karischen Neer westwïts bis ins Beringsmeer verbreitet, fehlen aber nach den bisherigen Beobachtungen am grössten 'Teil der sibirischen Küste (S. spinus zwischen der Karischen Pforte und der Beringsstrasse; S. boreas ist rom 
westlichen 'Teil des Karischen Meeres und rom östlichsten 'I'eil der sibirischen Küste bekannt). Man muss die Frage bis auf weiteres offen lassen, ob die Verbreitung an der sibirischen Kuiste unterbrochen ist oder nicht. Was S. spinus betrifft, ist es nicht unwahrscheinlich, dass die Lüicke in der Verbreitung scheinbar ist; das sibirische Eismeer ist noch weit von der Küiste so seicht, dass nur ein spärliches oder lokales Vorkommen zu erwarten ist; an den meisten bisher untersuchten Stellen könnte die Art unmöglich leben. S. boreas dagegen ist sonst überall so häufig in seichtem Wasser, dass man schon jetzt behaupten kann. dass ein auffallander Unterschied zwisehen dem Sibirischen Eismeer und andern arktischen Gebieten besteht; wenn die Art dort nicht fehlt, so muss sie jedenfalls sehr selten sein. Die Ursache dieser Erscheinung ist ganz unklar.

4. Diskontinuierlich zirkumpolare Arten: Verbreitung sowohl östlich wie westlich vom Nordmeer unterbrochen. Pandalus borealis und Eupagurus pubescens leben teils im Nordmeer und westlich davon bis Westgrönland, teils im pazifischen Gebiet. An den zwischenliegenden Küstenstrecken ist die Verbreitung nach den bisherigen Beobachtungen unterbrochen; $P$. borealis dringt nur selten ein wenig östlich vom Nordmeer in das Karische Meer ein; $E$. pubescens lebt an den Eismeerkisten in der Nähe der Beringsstrasse. In diesem F'alle hat man gute Grunde anzunehmen, dass die Verbreitung tatsächlich unterbrochen ist (s. unten). Möglicherweise gehört auch Hyas araneus zu dieser Gruppe (s. unten).

\section{Itlantische Arten.}

1. Westgrön land-Nordme er-Westsibirise he Arten. Hyas araneus und Sclerocrangon ferox sind von Westgrönland bis in das Karische Meer bekannt (der erstere nur bis in dessen westlichen und südlichen Teil), doch ist es ungewiss, ob sie diese beschränkte Verbreitung haben und nicht in Wirklichkeit zirkumpolar sind. $I$. araneus kommt möglicherweise auch im pazifischen Gebiet vor, obgleich er jedenfalls dort schr selten zu sein scheint (S. oben S. 66); er ist dann diskontinuierlich zirkumpolar. S. ferox kann, wie ApPeLiör (1906) und BIRULA $(1907,1910)$ hervorheben, sehr wohl an den Abhängen des Polarbeckens vorkommen; er gehört in diesem Falle zu den kontinuierlich zixkumpolaren, obgleich im Beringsmeer fehlenden Arten. Das Vordringen in den Stillen Ozean wird nach Brava durch die geringe Tiefe der Beringsstrasse und der See nördlich davon verhindert. Da die Art keine pelagischen Larven hat (s. WOLLEBæK 1908), dürfe diese Annahme richtig sein, doch muss man auch die Tatsache hervorheben, dass sie hocharktisch ist und daher unter keinen Umständen im Beringsmeer leben kömnte.

2. Nordmeerarten. Drei Decapoden sind nur vom Nordmeer bekannt: Bythocaris leucopis (nur in der Nordmeertiefe), B. payeri, B. simplicirostris (inkl. panschii) (Nordmeer von Nordostgrönland bis in das Barentsmeer). Da die beiden ersteren Arten auf mehr oder weniger grosse Tiefen beschränkt und hocharktisch sind (also nicht im Beringsmeer leben kömnen), muss man mit APPELLöF die Möglichkeit offen lassen, dass sie im Polarbassin vorkommen und folglich eigentlich zirkumpolar sind. B. simplicirostris ist so selten, dass die bisherigen funde wenig beweisen. - Von APpeluöf werden B. leucopis und payeri mit Sclerocrangon ferox zu einer Gruppe vereinigt, welche nicht ganz zutreffend östlich-arktisch genannt wird. 
III. Westliche Arten.

S'Tephensen (1912, 1913) unterscheidet eine Gruppe sendemische westgrönländische Litoralarten» und eine Gruppe »endemische grönländische Litoralarten »; in der Arbeit von 1913 werden die zuerst zu der zweiten Gruppe gerechneten Decapoden in die erste ïbergeführt. Diese Gruppe ist ziemlich heterogen und die Decapoden grösstenteils keineswegs in Grönland endemisch; die von STEPHensen nach CALman als marktischamerikanisch" bezeichneten Arten sind natürlich von den auf die Küsten von Grönland beschränkten Arten zu trennen (wenn solche überhaupt existieren). Die ganze Gruppe fällt mit der von APPELLöF unterschiedenen Gruppe von westlich-arktischen Arten zusammen.

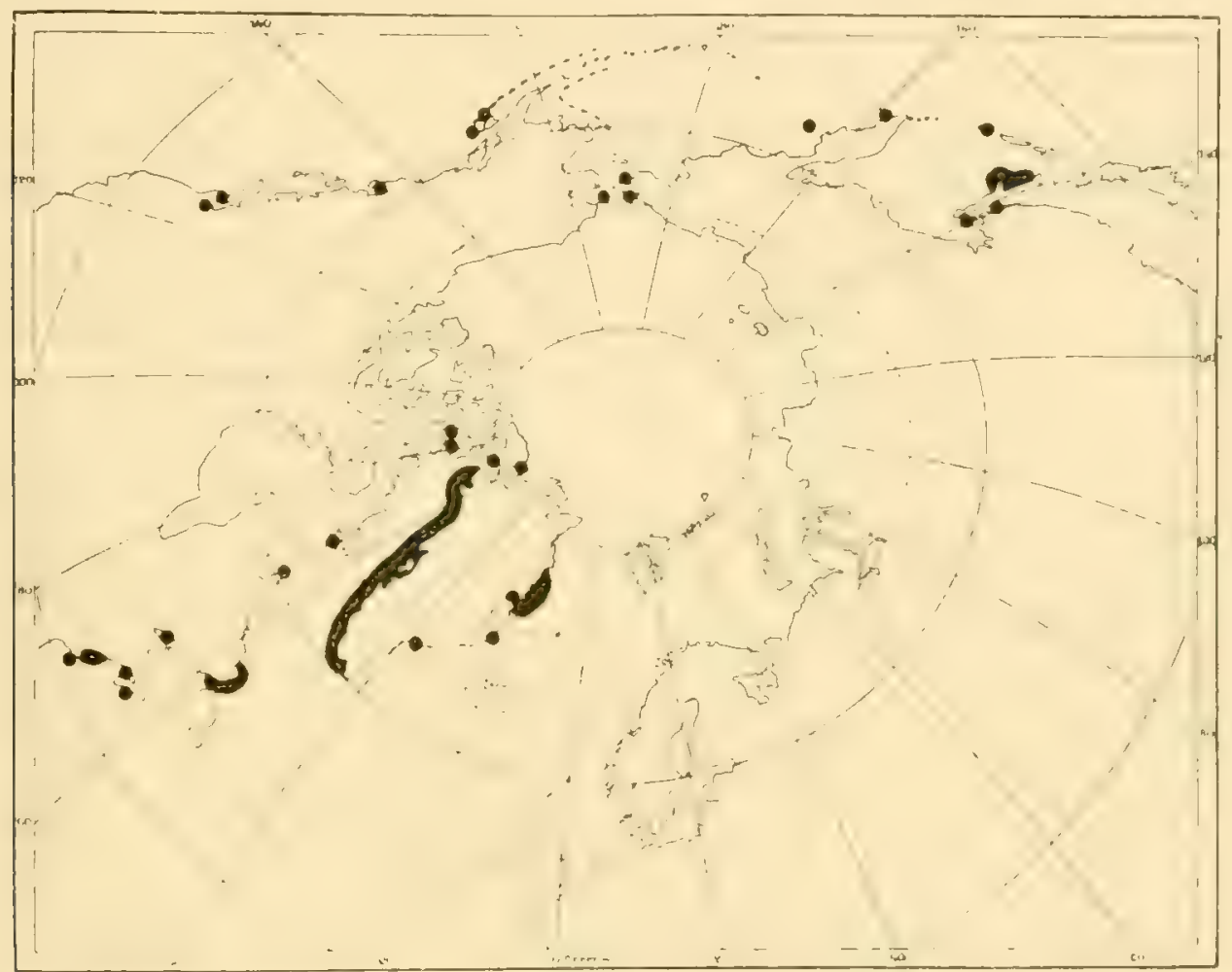

Fig. 12. Spirontocaris groenlandica (im pazifischen Gebiet ausserdom mehrere nicht auf einsclılägigen Kiarten zu findende Fundorte).

1. Nur von Westgrönland bekannt. Spirontocaris microceros (KR.) ist bisher nur an der Westküste von Grönland beobachtet worden, und dort nur in älterer Zeit. Ob sie nur in dieser und benachbarten Gegenden vorkomt, muss bis auf weiteres offen gelassen werden. Die neuerdings von MoLander (1914) beschriebene Art S. recurvirostris wïrde hieher gehören, ist aber zweifellos mit $S$. gaimardii identisch (s. oben $S .36$ ).

2. Pazifiseh-grönländische (pazifisch-nordamerikanische) Arten.

A. Spirontocaris groentandica (s. die Karte Fig. 12) und Nectocrangon lar sind von Nordostgrönland bis in das pazifische Gebiet verbreitet. (Die erstere Art wäre nach G. O. SARS einmal an der norwegischen Küste gefunden; diese Angabe ist aber zweifellos 
unrichtig; s. HANSEN 1908; Ross (1828) erwähnt die Art von Nordspitzbergen [Alpheus (culeatus FABR.]; auch diese Angabe muss natürlich als ganz unsicher bezeichnet werden.)

B. Chionoecetes phalangium (FABR.), Spirontocaris fabricii (KR.) (s. die Karte Fig. 13) und S'. macilenta (KRör ER) sind von Westgrönland und der Ostküste von Nordamerika bis in das pazifische Gebiet verbreitet; sie sind also noch ausgeprägter westlich als die vorigen Arten und dringen nicht in das Nordmeer hinein.

\section{P'azilische Arten.}

Die zahlreichen rein pazifischen Decapoden sind grösstenteils mehr oder weniger siidlich; ich nenne nur (nach RatiBun 1904) einige Arten, welche nördlich der Berings-

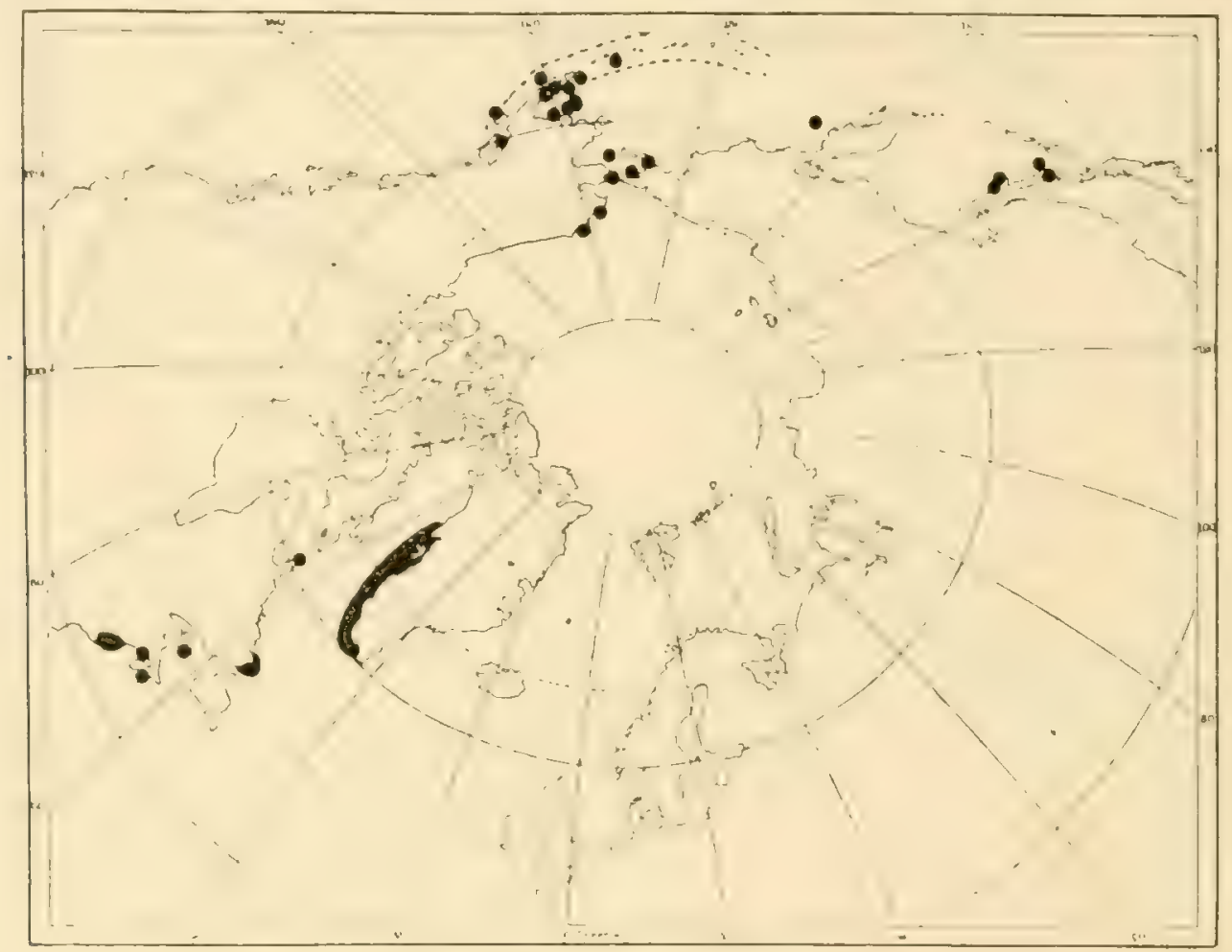

Fig. 13. Spirontocaris fabricii.

strasse, also unter unzweifelhaft arktischen Bedingungen, beobachtet worden sind: Spirontocaris murdochi RAтнв., S. dalli Ratuв., S. suckleyi (StтnPs.), S. camschatica (Stтміs.), Pandalus goniurus Stmps, Pagurus splendescens (OWEN). Diese Arten sind mehr oder weniger nahe mit zirkumpolaren Arten verwandt, besonders $S$. murdochi (mit S. spinus und lilljeborgii), S. suckleyi und camschatica (mit S. gaimardii).

\section{Die Ursachen der verschiedenen Verbreitung.}

Bei dem Versuch, die Unterschiede in der ost-westlichen Verbreitung zu erklären, begegnet man denselben Fragen und muss ron denselben Gesichtspunkten ausgehen wie bei den Echinodermen. Ich will hier nur die wichtigsten. Fragen kurz erörtern.

Die kontinuierlich. zirkumpolaren Arten ertragen hocharktische Bedingungen. Dagegen sind nicht alle hocharktische Bedingumgen ertragenden 
Arten zirkumpolar; einige (Sclerocrangon ferox und die Nordmeerarten) mögen zirkumpolar sein; die westlichen Arten Spirontocaris groenlandica und Nectocrangon lar sind es nicht. Über die wenigstens scheinbare Unterbrechung in der Verbreitung der zirkumpolaren Arten Spirontocaris spinus und Sclerocrangon boreas s. oben.

Die diskontinuierlich zirkumpolaren Arten. Pandulus borealis und Eupagurus pubescens sind Warmwasserarten. Diese Eigenschaft crklärt das Fehlen an den hocharktischen Kuistenstrecken zwischen dem atlantischen und dem pazifischen Verbreitungsgebiet; um die Zirkumpolarität zu erklären, muss man eine Verbindung in einer frïheren wärmeren Zeit annehmen (vgl. APPELLöF; iiber die Zeit der Verbindung s. auch meine Echinodermenarbeit). - Nach Birula (1910) beruht das Fehlen von $P$. borealis in den Küstengewässern Nordasiens darauf, dass dieses Gebiet zu seicht ist. Man kann jedoch mit ziemlicher Sicherheit annehmen, dass die Art auch in den tieferen Partien in grösserer Entfernung von der Küiste fehlt.

Wenn Hyas araneus im pazifischen Gebiet lebt, nimmt er ganz dieselbe Stellung wie die beiden zuletzt besprochenen Arten ein; wenn er dort fehlt, muss man vorläufig die Frage offen lassen, warum er im Gegensatz zu diesen nie bis dorthin vorgedrungen ist.

Uber die westlich en Arten hat sich Appeluöf geäussert (Stephensex [1912] bemerkt nur, dass einige Arten in ihrer Wanderung vom pazifischen Gebiet die Westküste, andere die Ostkiiste von Grönland »erreicht» haben; andere Arten »have come much further»; dies ist natürlich keine Erklärung). AppectöF erklärt die Ostgrenze dieser Arten dadurch, dass kein Strom vom westlichen zum östlichen Gebiet bekannt ist. Wahrscheinlich werden auch »die Wanderungen der erwachsenen durch die Stromrichtung beeinflusst»; einige westliche Arten sind zwar "in geringer Menge» an der Ostkiiste Grönlands gefunden worden, ses scheint aber, als ob sie dort keine richtige Heimat gefunden haben; sie scheinen nicht in genügender Menge iibergesiedelt zu sein, um sich einen festen Platz in der östlichen Fauna erobern zu können». Tch glaube, dass die Ursachen komplizierter sind und teilweise tiefer liegen.

Der Unterschied zwischen den an der Küste von Ostgrönland lebenden und den iibrigen westlichen Arten ist offenbar grösser, als ApPELLöF annimmt; die ersteren Arten. Spirontocaris groenlandica und Nectograngon lar, sind in Ostgrönland sehr allgemein, wie besonders aus den Angaben STEPHensen's (1913) hervorgeht. Der Unterschied zwischen den östlich von Westgrönland fehlenden und den auch in Ostgrönland lebenden Arten ist - wenigstens wenn man von der in Westgrönland vendemischen », in ihrer Verbreitung ganz ungenügend bekannten Art Spirontocaris microceros absieht - in der verschiedenen Thermopathie begrindet: diese sind arktisch-eurytherm, jene sind Warmwasserarten, welche hocharktische Bedingungen meiden und folglich unabhängig von den Verbreitungsmöglichkeiten im ganzen nördlichen Teil von Ostgrönland fehlen müssen (dass sie auch nicht vom südlichsten Teil der Küste bekannt sind, hat nichts zu bedeuten, denn dasselbe gilt von Eupagurus pubescens und Hyas araneus, und der grösste Teil dieser Kuiste ist faunistisch unbekannt). Es ist dies keine willkürlich gemachte Annalıme; Chionoecetes phalangium, Spirontocaris fabricii und S. macilenta sind in Westgrönland mehr oder weniger allgemein (wenigstens die beiden ersteren), sind aber weder von Nordwestgrönland, noch rom nordamerikanischen Archipel (wo Joness sund rerhälnismässign mut mter- 
sucht ist) bekannt, obgleich sie wieder im pazifischen Gebiet auftreten (STEPHensen [1912, S. 617-618] rechnet sie zu einer non the whole» borealen Gruppe, zu welcher allerdings auch einige in hocharktischen Gegenden häufige Arten gestellt werden).

Es erhebt sich dann die Frage, warum die beiden an der Küiste von Nordostgrönland allgemeinen Arten sich nicht weiter hinein in das Nordmeergebiet verbreitet haben. Die Antwort ist einfach: sie sind ausgesprochene Seichtwasserformen, welche die Einsenkungen zwischen Grönland und Island bzw. Spitzbergen ummöglich überschreiten können (Spirontocaris groenlandica: Ufer - etwa $100 \mathrm{~m}$, seltener in grösserer 'Tiefe, ganz ausnahmsweise sogar bis 350 und $500 \mathrm{~m}$; Nectocrangon lar: Ufer - $220 \mathrm{~m}$, in der Regel weniger, s. Hansen 1887, 1908, Ratibun 1904, Stephensex 1913). Als Larven wiirden sie vielleicht nach Nordisland gelangen können, aber jedenfalls nicht nach Spitzbergen (übrigens ist es nicht bekannt, ob sie pelagische Larven haben). Auch für die westgrönländisch-pazifischen Arten hat teilweise ein ähnlicher Gedankengang Geltung. Wenn sie auch nicht in Nordostgrönland leben kömmen, so stehen doch keine klimatischen Hindernisse ihrer Verbreitung nach Tsland im Wege, und von dort könnten sie sich ja sogar möglicherweise der boreoarktischen Zone des Färö-Island-Rückens und des Nordmeerabhanges entlang bis in den östlichen, arktischen Teil des Nordmeeres verbreiten; ferner ist es gar nicht unmöglich, dass sie in einer frïheren, wärmeren Zeit in Nordostgrönland gelebt haben. Auch diese Tiere sind jedoch Flachseearten (Spirontocaris fabricii: Ufer - etwa 100 oder 150 m; S. macilenta: 30 bis 50 - etwa $300 \mathrm{~m}$ ) oder wenigstens auf verhältnismässig unbedeutende Tiefen beschränkt (Chionoecetes phalangium: Ufer - etwa $450 \mathrm{~m}$, bisweilen bis etwa $600 \mathrm{~m}$; für diese und die obigen Tiefenangaben s. Hansen 1887, 1908, Stephensen 1913).

Die Westgrenze der westlichen Wärmearten ist eine natiirliche Folge ihrer Thermopathie; im sibirischen Eismeer können sie nicht leben (warum sie sich nicht früher dieser Kiiste entlang verbreitet haben, wissen wir nicht). Dagegen scheint es gegenwärtig unmöglich, eine Erklärung für die eigentuimliche T'atsache zu finden, dass die panarktischen, nördlich der Beringsstrasse lebenden Arten Spirontocaris groenlandica und Neclocrangon lar im Sibirischen Eismeer fehlen.

Auf die pazifischen Arten gehe ich hier nicht ein; sie scheinen teils Warmwasserarten zu sein, teils gehören sie den Formenkreisen von zirkumpolaren Arten an.

Uber die $\mathrm{H}$ er $\mathrm{k} u \mathrm{n} \mathrm{t}$ d e $\mathrm{r}$. A r t e $\mathrm{n}$ lässt sich nach der. Verbreitung allein nur wenig sagen. STEPHENSEx (1912) unterscheidet eine Gruppe von Arten, welche aus dem Stillen Ozean stammen und von dort der Nordkiiste von Amerika entlang gewandert sein sollen. Von arktischen und arktisch-borealen Arten bezeichnet er als solche Chionoecetes phalangium, Nectocrangon lar, Spirontocaris fabricii, macilenta, groenlandica, gaimardii und turgida. Die beiden letztgenannten Arten sind ja zirkumpolar; es ist nicht einzusehen, warum gerade diese zwei Arten und nicht auch andere erwähnt werden. Was die Herkunft dieser und anderer zirkumpolaren Arten betrifft, so erfordert diese Frage eine Prifung von ganz anderen Gesichtspunkten aus; wo sie auch entstanden sind, haben sie sich zweifellos sowohl ostwärts wie westwärts rings um den Pol verbreitet. Die iibrigen, pazifisch-grönländischen Arten stammen wahrscheinlich aus irgend einem 'Teil ihres heutigen Verbreitungsgebietes, und es liegt in der 'Tat nahe, zu vermuten, dass sie ursprünglich im Stillen Ozean zu Hause gewesen sind. 


\section{Die Verbreitung der Decapoden im Spitzbergengebiet.}

BrRuLA (1907) bespricht ausführlich die in den verschiedenen Teilen des Spitzbergengebiets herrschenden physikalischen Bedingungen. Er unterscheidet drei Zonen: 1. Die grossen und kalten Tiefen unterhalb des westspitzbergischen Golfstromastes. 2. Den vom Golfstromwasser bespülten Teil der Kontinentalstufe, von ungefähr 500 bis $150 \mathrm{~m}$. 3. Den übrigen Boden der Flachsee, nämlich Ostspitzbergen und die Fjorde Westspitzbergens. BiRULA bemerkt selbst, dass auch das Flachseegebiet des Westufers durch den Einfluss "des nahe durchgehenden Golfstromes" in seinen nallgemeinen arktischen Eigenschaften" verringert wird. Dieser Einfluss ist in der Tat sehr bedeutend; das Golfstromwasser dringt, wie z. B. die hydrographischen Beobachtungen unserer Expedition zeigen, auch in die Fjorde ein, obgleich es dabei allmählich abgekühlt und verdünnt wird. Man kann daher nicht West- und Ostspitzbergen zu einem gemeinsamen Gebiet vereinigen, sondern dürfte besser folgende Bezirke unterscheiden:

I. Niederarktische oder nur stellenweise hocharktische Ge bi e te.

1. Der von Golfstromwasser bespülte Teil der Kontinentalstufe von Westspitzbergen (in der Oberfläche gemischtes Küsten- und Golfstromwasser; nahe der Oberfläche der wenig bekannte spitzbergische Polarstrom). Hieher auch die Bänke südlich von Spitzbergen einschliesslich Beeren Eiland.

2. Die Fjorde der Westküste. Hocharktische Bedingungen (Wasser von konstant negativer 'Temperatur) nur in gewissen 'Tiefen oder am Grund tiefer, durch unterseeische Schwellen abgesperrter Fjordarme.

II. Ganz oder vorwiegend hocharktische Gebiete.

3. Die Nord- und Ostkiiste von Spitzbergen und das ganze, nur durch eine seichte Rinne vom Barentsmeer getrennte Flachseegebiet, im Suidwesten bis unweit von Beeren Eiland. Dieses Gebiet erhält sein Gepräge durch den östlichen eisführenden Polarstrom, und die Bedingungen sind daher überwiegend hocharktisch. Ganz rein hocharktisch ist jedoch nur der nordöstliche Teil. In den Storfjord dringt, wie die russischen Beobachtungen zeigen (KNIPowitscir 1905, S. 260, 1906 , S. 1445, vgl. auch die Angaben im russischen 'Text S. 1095-1097), wenigstens bisweilen abgekühltes Golfstromwasser ein, obgleich seine Verbreitung äusserst beschränkt ist. In einiger Entfernung von der Nordkiiste geht als Unterstrom die Fortsetzung des Westspitzbergstromes (Nansen 1906, Helland-Hansex \& Koefoed 1909, Knipowitseri 1906), - die Nordkuiste selbst ist dagegen natuirlich nicht, wie Röner \& Schaddry (1900) sagen, wausgezeichnet durch ihren Golfstromcharakter'»-, und ein wenig Wasser von niedrig positiver 'Temperatur dringt, wie schon KüKenthal und WALTER (KüKexthaL 1890) nachgewiesen haben, davon in die Hinlopenstrasse ein.

4. Die grossen und kalten Tiefen an der westlichen Kontinentalstufe.

Wenn man von den grossen Tiefen am westlichen Kontinentalabhang absieht, die

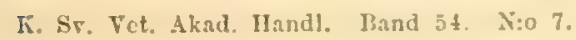


ja nicht zum eigentlichen Spitzbergengebiet gehören, kann man also nach den hydrographischen Bedingungen zwei Gebiete unterscheiden, einerseits Westspitzbergen und die Bänke südlich davon, andererseits Nord- und Ostspitzbergen (mit König Karls Land, Hopen Eiland und der Flachsee bis an die Grenze des Warmwassergebietes). Ungefähr dieselbe Einteilung haben schon Römer und ScmuUdins (1. c.) (und nach ihmen DofterN u. a.) benutzt, unter gleichzeitiger Hervorhebung des Fjordcharakters der westlichen und des Strassencharakters der östlichen Seite.

Hinsichtlich der Decapodenfauna findet DofLEIN (1900) einen Untersehied zwischen diesen Gebieten insofern, als die Spirontocaris-Arten im Sommer 1898 „ganz und gar auf das in jenem Jahre bedeutend eingeschränkte Kaltwassergebiet in ihrer Verbreitung begrenzt» waren. In Westspitzbergen seien sie an kalten Stellen rorhanden, »besonders im Hintergrund der Fjorde, und auch da nur in der Tiefe". "Fast genau so verhielt sich Pandalus boreatis, ähnlich, wenn auch nicht ganz so streng, Hyas araneus, Sclerocrangon boreas und Sabinea septemcarinata». Diese Ansicht bedarf keiner Widerlegung. Zwei der besprochenen Arten (Pandalus boreatis und Hyas araneus) sind Warmwassertiere, die im Osten sehr selten sind. Die ibrigen sind panarktisch (arktisch-eurytherm) oder arktisch-boreal und sehr allgemein im ganzen Spitzbergengebiet (s. näheres im Speziellen Teil). Nur ist es möglich, dass einige Arten (besonders Spirontocaris polaris) in Ostspitzbergen häufiger in ganz seichtem Wasser sind.

Birula (1907) hat einen Vergleich zwischen der Decapodenfauna von West- und Ostspitzbergen angestellt und ist zu dem Ergebnis gekommen, dass ein Untersehied ausschliesslich in dem Vorkommen einiger borealen Arten in Westspitzbergen besteht. Dies ist nicht richtig; es gibt andere Unterschiede, die sogar wichtiger sind (der von BIRULA hervorgehobene Unterschied verliert an Bedeutung dadurch, dass die borealen Arten kaum konstante Mitglieder der westlichen Fauna sind und nicht in die Fjorde einzudringen scheinen). In den nachstehenden Bemerkungen bericksichtige ich nicht die kalte Area an dem Abhang der Westkuiste, wo die Fauna mit derjenigen der ibrigen Nordmeertiefe ïbereinstimmt; man findet dort die hocharktischen Arten Sclerocrangon ferox, Bythocaris leucopis und B. payeri, ferner B. simplicicrostris, dagegen gibt es - im Gegensatz zu anderen Tiergruppen - keine fuir die Tiefsee und die westliche Flachsee gemeinsamen Decapoden (wenn nicht die letzterwähnte Art auch das letztere Gebiet bewohnt).

Die Gesamtzahl der im Spitzbergengebiet heimischen benthonischen Decapoden beträgt 10; dazu kommen drei soeben erwähnte nur in der Tiefsee gefundene Arten und 4 boreale Gäste. 6 dieser Arten sind sicher oder wahrscheinlich i $m$ g a $n$ z e $n$ G e b i e t verbreitet und überall häufig; es sind dies Spirontocaris polaris, S. gaimardii, S. spinus, S. turgida, Sclerocrangon boreas, Sabinea septemarinata. Linige dieser Arten sind zwar vorwiegend an der Westkiiste und im siidwestlichen 'I'eil von Ostspitzbergen gefunden worden, nach ihrer ibrigen Verbreitung muss man jedoch wenigstens bis auf weiteres annehmen, dass sie iiberall vorkommen.

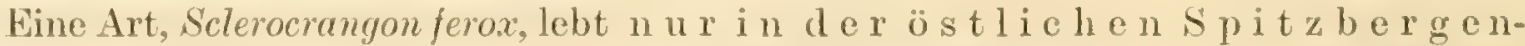
s e e und a n d e r Nordk iis t e ; an der Westkiiste tritt sie erst in der kalten 'liefenarea auf.

Unter den an der Westküste wirklich heimischen Arten gibt es keine, welche voll- 
ständig auf dieses Gebiet beschränkt ist. Die $t$ n u r in W estspitzbergen beobachteten Decapoden, Hyas coarctus, Sabinea sarsii, Pontophitus norvegicus und Lithodes maja, sind boreale Arten, welche nur als zufällige Gäste oder wenigstens sehr selten an der Westseite auftreten (die letzerwähnte Art ist sogar nicht nördlich von Beeren. Eiland, $P$. norvegicus nicht nördlich vom Südkap beobachtet worden).

Dagegen gibt es drei Arten, welche v o r w i e g e n d in West s p it z b e r gen zu Hause sind, nämlich die niederarktisch-borealen Warmwasserarten Pandalus boreatis. Hyas araneus und Eupagurus pubescens. Diese Arten sind im Osten nur oder fast nur im siidwestlichen 'Teil des Gebietes (Storfjord und südlich davon) gefunden worden; die letztgenamnte Art scheint dort verhältnismässig allgemein zu sein, die beiden iibrigen sind mehr oder weniger selten.

Der Storfjord und die Nordküste der Westinsel sind faunistisch Ubergangsgebiete zwischen der Westküste und dem rein hocharktischen Nordostgebiet; man findet hier sowohl die hocharktische Art Sclerocrangon ferox wie die soeben erwähnten Warmwasserarten. 


\section{Anmerlinngen zu den Fundortsrerzeichissen.}

I 24. und 25. 7 an zwei hydrographischen Stationen in der Srensksundstiefe in $300 \mathrm{~m}$ Tiefe: Temp. $+2,42^{\circ}$, bzw. + 2,51 , Salzgeh. 34,85 bzw. $34,88 \%$

II 5. $8,138 \mathrm{~m}$ Tiefe: $-0,51^{\circ}$.

III Nach mehreren Beobachtungen an anderen Stellen in der safe $13 a y$ am 15.7 und 5 . 8 .

IV Sieho die IIylrographischen Ergehnisse (in etwa 100, meist in $60-140 \mathrm{~m}$ 'Tiefe fast überall negative "Temperotur oder wenigstens etwa $\left.0^{\circ}\right)$; vgl, anch St. 20 (55 $\left.\mathrm{m}:-0,28^{\circ}\right)$.

$\checkmark$ In der Nähe von St. 24 , am 21. $7:+5,6^{\circ}$.

VI Wahrscheinlich; nach Beobachtungen 0 . von st. 30 , am 17.7 .

III siehe dio Hydrographischen Ergebnisse.

VIII Nach Vessungen SW. ron St. 91 und in der Hitte der Ekman Bar, am 19. und 20. 8.

IX No. ron st. $117,30 \mathrm{~m}, 26.8:+2,01$

I No. ron st. 121 .

II SW, von St. 122, 26. $8 ; 40 \mathrm{~m}:-0,13^{\circ} ; 50 \mathrm{~m}:-1,6^{\circ}$.

XII Insserer Teil der Dickson BaF, 26. $8 ; 0 \mathrm{~m}:+3,62^{\circ} ; 10 \mathrm{~m}:+3,82^{\circ}$.

XIII In der Nähe von St. 76 und 77.

$\mathbb{X I V}$ Siehe die Iydrographischen Ergebnisse (in etwa 100, meist 60-140 m fast überall negative Temperatur oder wenigstens etwa $0^{\circ}$ ); rgl. Anm. IV.

IV Unweit von St. $79,27 \mathrm{~m}, 12.8:+1,82^{\circ}$

XVI Etwas No. von St. $81,12.8$.

XVII Eingang der Billen Bay, $27 \mathrm{~m}, 12.8:+1,82^{2}$.

XYII Tach Yessungen am Eingang und im inneren 'Teil der Billen Bay, 12. und 17. 8.

XIX Nach Ilessungen im Ostarm und im Fingang der Billen Bay.

XX Wahrscheinlich (nach den Temperaturrerhältnissen der St. 51 und 56 zu urteilen).

XXI Nach Messungen NO. von St. 72, 28. 7.

XXIl Nach Messungen am Fingang und in der Mitto der Adrent Bay, 27. und 28. 7.

XXIll Nach mehreren Messungen im Eingang der Advent Bay, im Ost-und Nordarm, 27. und 30. 7, 29. 8.

XXIV Etwas NW. ron St. $69,75 \mathrm{~m}, 18.7:+1,69^{\circ}$

XXY Nach Messungen in der Oberfläche und am Eingang der Coles Bay (18.7) zu urteilen.

XXYI Nach Messungen etwas N. ron St. 126 und 127, 22. 8.

XXVII Nach Messungen in der Oberfläche, 18. 7. 


\section{Literaturyerzeichnis.}

Adais, A., 1852. Decapoda. In: Sutherland, P. C., Journ. of a Voy. in Baffin Bay and Barrow Straits $1850-51$. Vol. 2. Append. (London).

Andrews, W., 1878. Notos on Irish Crustacea, I. Sc. Proc. R. Dubl. soc. (N.S.) Tol. 1.

APPELLöF, A., 1905. Havbundens dyreliv. Norges Fiskerier, I, 1. (Bergen).

, 1906. Die dekapoden Crustaceen. Meeresfauna von Bergen, H. 2 u. 3. (Bergen). - Angahen üher das Vorkommen der Arten in früheren Mitteilungen (Berg. Mus. Aarb.) habe ich nicht zitiert.

1909. Untersuchungen über den Hummer. Bergens Mus. Skrifter. (N. R.) Bd. I. N:o 1.

1912. Invertebrato bottom Fauna of the Norwegian Sea and North Atlantic. In: J. MURR.Y and J. HuorT, The Depths of the Ocean. (London).

1912 a. Über die Beziehungeh zwischen Fortpflanzung und Verbreitung mariner Tierformen. Verh. d. VII. Int. Zool.-Kongr, zu Graz 1910.

AURIvillius, C., 1886. Ifafsevertebrater frủn nordligaste 'Tromsö amt och Vestfinmarken. Bih. t. K. Svenska Vet.-Akad. Handl. Bd. 2 (Afd. 4). (Stochholm).

1889. Die Maskierung der oxyrhynchen Dekapoden. K. Svenska Vot-Akad. Handl. B1. 23.

1898. Om hafsevertebraternas utvecklingstider ete. Bihang. t. K. Srenska Vet.-Akad. Hamil. Bd. 24 (Afil.4).

Awerinzew, S., 1909. Einigo Beiträgo zur Verbreitung der Bodenfauna im Kola-Fjorde. Int. Rev. ges. IIydro. biol. Bd. 2 .

\section{Miirekex}

BALSS, H., 1913, 1914. Ostasiatische Decapoden I, II. Abh. Akad. Wiss. Hiey, 2. K1., 2. Suppl.-B1.

BATE, Sp., 1888. Report on the Crustacea Macrura collected by H. M. S. Challenger. Challeng. Rep., Zool., Vol. 24.

BELL, TI., 1853. A History of the British stalk-ejed Crustacea. (London).

- - 1855. Account of the Crustacea. In: Belciner, The last of the arctic Voyages. (London.)

VAN Beneden, P., 1861. Recherches sur la Faune littorale de Belgiquo. Crustacés. Mém. Acad. Bclo. T. 33.

Bidenkar, A., 1899. Undersögelser over Lyngenfjordens Lvertebratfauna. Tromsö Mus. Aarsh. 20 (1897).

Birula, A., 1897. Recherches sur la biologio et zoogéographie, principalement des mers russes. III. Essai d'unc faune des crustacés décapodes de la mer Blanche et Mourmanne. Anu. Mus. zool. Acad. Sci. St. Pétersbourg. T. 2. (Russisch.)

1899. Recherches etc. VI. Décapodes recueillis dans la mer Mourmanno 1898 par l'expédition du Comité des Ponores. Ibid. T. 4. (Russisch.)

1900. Recherches etc. VII. Notes sur les Crustacés recueillis... en 1896 et 1897, dans la Mer de Kara ete. Ibid. T. 4 (1899). (Russisch.)

- 1907. Zoologische Ergebnisse der Russischen Expeditionen nach Spitzbergen. Crustacea-Decapoda. Ibicl. 'T. 11 (f. 1906).

1910. Beiträge zur Kenntnis der Decapoden. T́rebse der eurasiatischen Aretis. Mém. Acad. imp. d. Sciene. St. Pétersbourg. (8) T. 29, N:o 1 (Rés. scient. do l'Expéd. Pol. Russo on 1900-1903, Sect. L: 'Zool., Vol. 2, livr. 1.

BJöRck, W., 1911. Bidrag till kännedomen om nordhafsräkans (P'andalus borealis Kír.) uthredning och biologi Kattegatt och Skagerack. Svenska Hydrogr. Biol. Kommiss. Skrift. Bd. 4.

1913. Decapoden aus dem Kattegatt und dem Skagerak. Ark. f. Zoologi (K. Sw. Vet.-Akad.). Bd. 8, X:o 3. (Stockholm).

1913 a. Biologiseh-faunistische Untersuchungen aus dem Öresund I. Pantopoda, Mysidacea und Decapoda. Lunds Unir. Arsskrift. (N. F.) Afd. 2. Bd.9 (K. Frsiogr-Sällsk. IIandl, N. F, Bd. 24).

- - 1915. Biologisk-faunistiska undersökningar af Öresund II. Crustacea malacostraca och Pantopoda. Ibill. Bd. $11(26)$.

Brandt, F., 1851. Krobso. Middendorff's Reise in den äussersten Forden und Osten Sibiriens. IBd. 2, 'Zoologie, Teil 1. (St. Petersburg.) 
Brasmikow, W., 1907. (Beiträge zu Fauna der russischen, östlichen Meere, ges. v. U. Schoner Staroseh 189?1902.) llém. Acad. Sci. St. Pétershourg. (8) Vol. 20. (Russisch.)

Breitrus, Is., 1903. Exped. f. wiss.-prakt. Unters, an d. Murmanküste. Ber. üb. d. 'Titigk. pro 1902. (St. Pétersb.) Buchnolz, 1874. Crustaccen. Dio 2. dentsche Sordpolarfahrt 1869 u. 1870. Bd. ‥ Wiss. Lirg. (Leipzig.) Colgax, X., lgo5. Notes on the invertehrate fauna of Skerries, Co Dnblin. Irish Natur. Vol. 14.

Colietr, R., 1905. Fisko indsamlede under "Michael Sars's \& Togter i Nordhavet 1900-1902. Sorw. Fish. 2, $\mathrm{N}: 03(1909)$. (Bergen.)

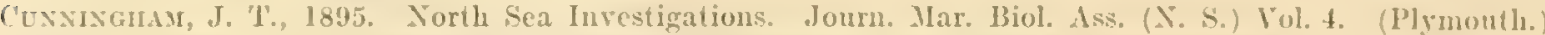

v. DAlta ToleE, K. W., 1889. Die Fauna von Helgoland. Zool. Jahrh. Suppl. Heft 2.

DALYELL, J. (G., 1851. 'The Powers of the Creator. Vol. I. (London.)

DANTELssex, D. C., 1859. Beretning om en zoologisk Reise i Sommeren 1858. K. Jorko Vidensk. Selsk. Skrifter. Bd. 4. (Trondhjem.)

1861. Borotning om en zoologisk Reiso foretagen i sommeren 1857. Nyt Magaz. f. Naturs. Bd, 11. (Kris(iania.)

DErJugix, K. M., 1906. [Murmansche Biologische Station 1899-1905.] Trav. Soc. nat. zool. St. Pétersburg 37. Livr. 4. (Russisch.)

191. Dio Murmansche Biologische Station. Proc. 7. int. zool. Congr. Boston 1907. (Cambr., Mass.)

DOFteiv, F., 1900. Dio dekapoden Krebso der arktischen Meere. Fanna aretica. Bd. I.

-, 1902. Ostasiatische Decapoden. Abh. Barr. Akad. 21, Abt.3.

Doxs, C., 1913. Slegten IIyas, 'Tromsö Mus. Aarsh. 3t (1911). 1915. Nord-Norges Decapodor. Ibid. 37 (1914).

D'Urban, W. S. II., 1880. The Zoology of Barents Sea. Ann. Mag. Nat. Hist. (5) Vol. 6.

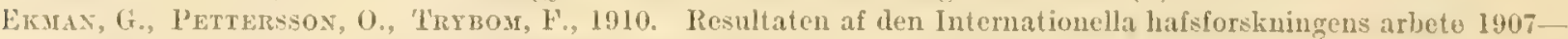
1909. Skrift. utg. af Kgl. Jordbruksdepart., Meddel. 22. (Stockholm.)

FABricius, 0. F', 1780. Fauna groenlandica. (Hafnix et Lipsis.)

Falrax, G. 1'., 1912. Clare Isl. Surv. Decapoda. P'roc. R. Irish. Acad. Vol. 31. (Dublin.)

Forsstraxd, C., 1886. Det arktiska liafsområdets djurgeografiska begränsning med ledning af skalkriftoruas (Crustacea malacostraca) utbredning. (Uppsala, Diss.)

EULTON, 1. W', 1890, 1891, 1893, 1894, 1897, 1898. Roports on the trawling oxperiments of the "Garland pete. Ann. Rep. Fish. Board f. Scotl. 8 (f. 1889), 9 (f. 1890), 11 (f. 1892), 12 (f. 1893), 15 (f. 1896), 16 (f. 1897 ).

Gaistaxg, W., 1892. Notes on the Marine Invertebrate Fama of Plrmouth f. 1892. Journ. Mar. Biol. As. Vol. 2. 1905. Report on the trawling investigations 1902-03. Mar. Biol. Ass., 1. Rep. on Fish. and hydrour. Inrest. in tho North Sea (southern area). (North. Sea Fish. In rest. Comm., Rep. X:o 2). (London.)

GoËs, A., 1863. Crustacea decapoda podophthaha marina Suecine ete. Ofvers. K. Svenska Vot.-Akad. Förhandl. 20. (Stockholm.)

Grieg, J., 1909. Invertébrés du fond. Due d'orléans, Crois. Océan. d. 1. Balgica i90J. (Bruxelles.)

- -, 1914. Bidrag til kundskapen om Hardangerfjordens fauna. Bergens Mlus. Aaarb. f. 1913.

HAxsex, H. J., 1887. Malacostraca marina Groenlandia oceidentalis. Vidd. Meddel. Naturh. Foren. Koben. havn 1887 .

_-, 1887 a. Oversigt over de paa Dijmphna-Togtet indsamlede Krelsedyr. Dijmplna-Togt. zool.-bot. Udb. (Köbenhavn.)

, 1895. Pyenogonider og Malacostrake Krebsdyr. Den östgrönl. Exped. 1891-92, 3. Del. Meddel. om Grönland. II. 19. (Köbenhavn.)

- 1908. Crustacea Malacostraca (I.). The Dan. Ingolf-Exped. Tol. III, P. 2. (Köbenhavn.)

- 1909. Note on the Crustacea. Carkshorgsfonil. Exped. to Ost-(Frönland 1898-1900. Meddel. om Grönland. II. 29, Afd. 2. (Köbenhavn.) (Auszug ans der vorigen Arbeit.)

HARTLAUB, C., 1900. Zool. Ergebn. einer Untersuchungsfahrt des dentsch. Seofischerei-Ver. 11. A. Bäreninsel und Westspitzbergen 1898 auf S. If. S. „Olga». Einleitung. Wissensch. Meeresunters. (N. F.) Ba. 4. Abt. Holgoland. (Kiel u. Leipzig.)

IIAssat, A. II., 1842. A list of Invertebrata found in Dublin Bay and its vicinity. Ann. Mag. Yat. Hist. (1) Yol. 9.

IIEAPE, W., 1888. Preliminary Report upon tho Fauna and Flora of Plymouth Sound. Journ. Mar. Biol. Ass. Vol. 1. (Lomdon.)

Helland-Haxsex, B., et Koefoed, E., 1909. Hydrographic. Duc d'Orléans: Crois. océan. 1905. (Bruxelles.)

IIfleand-Haxsex, B., \& Ninsex, F., 1909. The Torwegian Sea etc. Rep. on Norw. Fish. and Mar. Invest. Yol. 2. I'. 1 .

Hellek, C., 1875. Dio Crustacen, Pyenogoniden und Tunicaten der K. K. Ostorreich-ungar. Nordpol-Exped. Denksehr. Akal. Wiss. Wien, Math.-naturw. Kl. Bd. 35.

IIEnderson, J. K., 1886. A. Synopsis of British Paguridie. Proc. R. Phys, Soc. Fdinb. Vol. 9. $1885-88$.

, 1887. 'The decapod and seizopod Crustacea of tho Firth of Clyde. Proc. and 'Trans. Yat. Hist. Soc. Glasgow. (N. S.) Vol. 1. 1885-86. 
Heromax, W. A., 1896, 1901. Ninth, Fourteenth annual Report of the Liverpool Biol. Comm. Proc. Trans. Li verp. Biol. Soc. Vol. 10, 15 .

HEY, W. O., 1903. Shoro-collecting at Scarborough and Filer. Naturalist 1903.

HJortir, J. \& DanL, K., 1900. Fisling Experiments in Norwegian Fiords. Rep. Norw. Fish. and Mar. Inv. Vol. 1. (Kristiania.)

HoEk, I. P. C., 1882. Die Crustaceen gesammelt wilhend der Fahrten des "Willom Barents» 1878 und 1879 Nioderl. Arch. f. Zoologie. Suppl.-B3d. 1 (1881-82).

-_, 1883. Sehaaldieren van de Oosterseholde (Crustacés de l'Escaut de l'list). 'Tijdschr. Nederl. Dierk. Vereen. Suppl. D. I, גfl. 2.

IIOLT, E. W., 1892. Surrey of Fishing Grounds, west coast of Ireland, 1890-91. Sc. Proe. R. I)uhl. Soe. (N. S.) Vol. 7 .

IÏ̈ring, R., 1902. Rapport om F'iskeriundersögelserne under Faröeno og Island i Sommeren 1901. F'jskeri. Beretı. f. 1900-01. (Köbenhavn.)

How E, F., 1901. IReport of a Dredging Expedition off tho Southern Coast of Now Lngland, Sept. 1899. Bull. U. S. Fislı. Comm. Tol. 19.

Hoxte, W. E., 1890. On the Deep-water Fama of the Clyde Sea-Area. Journ. Limn. Soc. London, Zool. Vol, 20.

JARZYNsKr, TH., 1885. Premissus Catalogus Crustacearum decapodum, inventarum in mari allo et in mari glaciali ad litus murmanicum anno 1869 et 1870. Anhang zu WAGNer 1885

JoInstone, J., 1905. 'Trawling observations and results. Proc. Trans. Liverpool biol. Soc. Yol. 19.

KeMP, S., 1910. The Decapoda Natantia of the coasts of Ireland. Departm. of Agric and techn. Instr. f. Ireland, Fish. branch, Sci. Inv, 1908, N:o 1. (zitiert nach STAPPERS).

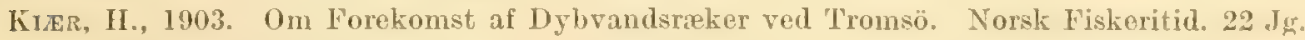

1904. Dyreliret i Dröbaksund. Nyt Mag. f. Naturvid. Bd.42. (Kristiania.)

$1906((\$ 1905-06 »)$. Om Tromsösundets fiske. 'Tromsö Mus. Aarsheft. 27 (1904).

1906 a ("1906-07»). Om dyrelivet i Balsfjorden. Ibid. 28 (1905).

Kinaman, J. R., 1859. Report on Crustacea of Dublin District, P. 1. Rep. Brit. Ass. 28. mect. 1858.

Kingstey, J. S., 1901. Preliminary eataloguo of the marine Invertebrata of Casco Bay. Proc. Portland. Soc. Nat. Hist. Yol. 2.

ḰLinckowströß, A., 1892. Öfversigt af de zoologiska arbetena under expeditionen till Spetsbergen 1890. [G. Nordenskiold, Redog. f. d. Sr. Exped. t. Spetsb. 1890. Bil. V1.] Bih. t. K. Sr. Vetensk.Akad. Handl.. Bd. 17 (afd. II). (Stockholm.)

KNıPowitscir, N., 1901. [Arbeit nit nur russischem 'Titel ïber die Ergebnisse der Dredgungen des "Ermak" 1901.] Bull. Acad. sci. P'étersburg. (5) '1. 6.

_- 1905. Iydrologisehe Untersuchungen in Europäischen Eismeer. Aunalen d. Hydrogr. u. marit. Meteorol. Jg. 33. (Berliu.)

1906. Grundzüge der Hydrologie des Europäischen Eismeeres. Zap. Russ. geogr. obsc. T' 42. (St. P'etersburg.)

Koenler, R., 1886. Recherehes sur la Faune marine des lles Anglo-Normandes. Bull. Soc. Nancy (2) 7 . Siehe auch Ann. sci. nat. (6) Tol. 20.

Koelbet, C., 1886. Crustaceen, Pyenogoniden und Arachnoiden von Jan Mayen. Die intern. P'olarforsch. 188283. Die österreich-Polarstat. Jan Mayen, Beob.-Ergebn. Bd. 3. (Wien.)

Krörer, H., 1838. Conspectus Crustaceorum Groenlandix. Naturh. Tidsskr. Bd. 2. (Köheuharn.)

- -, 1842. Monografisk fremstilling af sligten Hippolytes nordiske arter. (Köhenharn.) 1843. Do hidtil bekjendte nordiske Krangon-Arter. Naturh. Tidsskr. Bd. 4.

Kükextial, W., 1890. Forschungsreise in das europäische Eismeer 1889. Dentsehe geogr. Blätt. Bd. I3.

LAgerberg, T., I908. Sreriges decapoder. Göteborgs K. Vetensk. o. Vitterh. Samlis Handl. (4. F.) H. 51. N:o 1. (Göteborg.)

LEACII, W. E., 1815. Malacostraca podlophthalmata Britanniae (illustr. by J. SonERBY). (London.)

LENZ, H., 1901. Ergebnisse einer Reiso nach dem Pazifik (Schaninslant). Crustaceen. Kool. Jahrb. Aht. f. Syst. Bd. 14.

Lestie, G., \& Herdax, W. A., 1881. The Invertebrate Fauma of the Firth of Forth. P. 11. P'roe. R. Phys. Soe. Edinburgh. Vol. 6 .

LiluJeborg, WV., 1851. Bidrag till Norra Rysslands och Norriges fauna. K. Srenska Vet.-Akad. Hand!. 1850. (Stockholm.)

_-, 1852. Hafs-Crustaceer vid Kullaberg. Öfrers. K. Sv. Vetensk.-Akad. Förh. Bd. 9.

LöNvBerg, E., 1898. Undersökningar rörande Öresunds djurlif. Meddel. fr. K. Idand bruksstyrelsen N:o I ir 1898 (N:0 43). (Uppsala.)

- - 1903. Undersökningar rörando Skoldervikens och angränsando Kattogatt-omrâdes djurlif. Ibid. N:0 보 $1902(\mathrm{~N}: 0 \mathrm{8} 8$ ).

LUADBECK, $11 ., 1893$. Beretning om Fisheriundersögelser og dermed forbundne zoologisko Indsamlinger paa do islandske Fjordo i Sommeren 1893. Fiskeriberetning f. 1892-93. (Kübenhavn.) 
Inrine Biologinal Association 1909. Trawling investigations, 1904-05. I ("Iuxlern). Mar Biol. Ass. Int. Fish. Invest., 2. Rep. (South. Area) on Fish. and hydr. invest. in the North Sea 190t-05. P. II (= North sea Fish. Invest. Comm., 2. Rep. ete.) (London.)

MAssy, 1. L., 1912. Report of a Survey of 'Trawling Grounds ete. Fish. Ireland sci. Invest. 1911. I.

Metnert, I', 1877. Crustacea isopoda, amphipoda et decapoda Daniae. Naturh. Tidskr. (3. 1R.) Bd. 11. (Kö. henhavn.)

1890. Crustacea malacostraca. Det vidensk. udbytte af Kanonb. oHatichs \# togter 1883-86. Bd. 3. (Kö. henlavu.)

Metzger, A., 187\&. Crustaceen aus den Ordnungen Edriophthalmata und Podophthalmata. (\%ool. Lrg. d. Nord. seefahrt 1872, X:0 10). Jahresb. d. Komm. z. Unters. d. dentschen Yeere in Kiel. 2. (Berliu.)

IIErs, L., 1877. List of the Species of Crustacea collected by the Rev. A. F. Faton at Spitzhergen in the summer of 1873. Ann. Mar. Nat. Hist. (1) Vol. 19.

, 1877 a. Report of the Crustacea collected by the Naturalists of the Aretic Expedition in $1875-76$. Ibid. (4) Vol. 20. Auch in: Nares, Voyage to the Polar Sea 1875-76, Appendix. (London, 4. Ed. 1878.)

, 1880. On a small Collection of Crustacea made... chiefly in the $\mathrm{N}$. Greenland Seas etc. Journ. Lim. Soc., Kool. Vol. 15 (f. 1881).

, 1881. On a small Collection of Crustacea and Pycnogonida from Franz-Josef Land. Ann. and Ifag. of

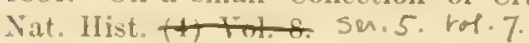

MILNE-EDWARDs, H., 1834-40. Histoire naturelle des Crustacés. (Paris.)

Milne-EDWards, A., \& Bouvier, E. L., 1894. Crustacés decapodes provenant des eampagnes du yacht l'Hirondello $(1886,1887,1888)$. Pt. I. Rés. camp. Albert I do Nonaco Fasc. 7.

M'Inтовсі, W. C., 1875. The Marine Invertebrates and Fishes of St. Andrews. (Edinburgh.)

MöвıUs, K., 1873. Die wirbellosen Thiero der Ostseo. (Ber, üb. d. Exped. z. phys.ehem. u. biol. Unters. d. Ostsee 1871 auf S. .I. Ponmerania.) Jahrésb. d. Kommis, z. wiss. Unters, d. deutseh. Neere in Kiel. 1. Jg. f. 1871. (Berlin.)

1893. Über die Thiere der Schleswig-Holsteinischen Austernbanke. Sitzber. Akar. Wiss. Berlin 1893.

Molander, A., 1914. Two new species of Decapods. K. Sv. Vet.-Akad., Ark. f. zool., Bd. 9. (Stockholm.)

MURDocu, J., 1885. Marine Invertebrates (exelusive of Mollusks). Rep. of the intern. Polar Exped. to P. Barrow, Mlaska. (Washington.)

N.ANSEx, F., 1906. Northern Waters. (R. Amundsens Oceanogr. Observ. in the Aretic Seas in 1901.) Vidensk. selsk. skrifter 1906. I. Math.-naturv. Kl., N:o 3. (Kristiania.)

FordGatri, 0. 1905. Hydrographical and biologieal Investigations in Yorwegian Fiords. Bergens Iluseums Skrifter.

- -, 1912. Faunistiske og biologiske iakttagelser ved den Biologiske station i Bergen. K. Norske Vidensk. selsk. Skritter 1911. N:0 6. (Trondhjem.)

Nonvax, 1. II., 1862. On the Crustacea... obtained ... off the Shetland Isles in 1861. Rep. Brit. Ass. f. 1861.

- - 186\%. Rep. of the Comm.... Explor.... Hebrides. I. II. On the Crustacea etc. Ibid. f. 1866.

1869. Shetland Final Dredging Report. Part. II. On the Crustacea, Tunicata, Polyzoa, Echinodermata, Aetinozoa, Hydrozoa, and Porifera. Ibid. f. 1868.

-, 1877. Crustacea otc of the "Valorous" Cruiso. Proc. R. Soc. Isondon. Yol. 25.

, 1894. I IIonth on the Trondhjem Fiord. Ann. Mag. Nat. Hist. (6) Vol. 13.

-, 1902. Notes on the Natural History of East Finmark. Ibid. (7) Vol. 10

. 1907. Notes on the Crustacea of the Channel Islands. Ibid. (7) Vol. 20

1909. The Celtic Province: its extent and its marine Fama. Trans. Ifertfordshire Nat. Hist. Soc. Vol. 14, I', 1 .

Fonst, A. II., \& Brapr, G. S., 1911. The Crustacea of Northumberland and Durhan. Trans. Neweastle Nat. Hist. Soc. Vol. 3., Pt. 2.

NonMaN, A. M., \& ScotT, Tri., 1906. The Crustacea of Devon and Cornwall. (London.)

OHLIN, 1., 1895. Bidrag till kännedomen om malakostrakfaunan i Baffin Bay och Smith Sound. Acta Univ. Lund. Bd. 31 (K. Fysiogr. sällsk. handI, X. F. Bd. 6).

- 1901. Arctic Crustacea collected during the swedish arctic expeditions 1898, 1899 and 1900. II. Decapoda, scizopoda. Bihang t. K. Sv. Vet.-Akad. Handl. Bd. 27, afd. IV. (Stockholm.)

ÖRsted, A. S., 1844. De Regionibus Marinis. (IFamix.)

, 1845. Fortegnelse over Dyr, samlede i Christianiafjord red Dröbak. Nat. Tidsskr. (2) Bd. 1, $1844-45$.

Ortann, A., 1890. Die Decapoden-Krebse des Strassburger Mruseums, I. Zool. Jahrb. Abt. f. Syst. Bd. 5 (f. 1891).

-, 1896. Grundzüge der marinen Tiergeographie. (Jena.)

, 1901. Crustacea and Pycnogonida coll. dur. the Princeton Exped. to Forth Greenland. Proc. Acad. Nat. Sci. Philadelphia 1901.

-, 1901 a. Crustacea, 2: Malacostraea. Bronns Klass. U. Ordn. d. Tierr. Bd. 5, II. Abt. 
PACKAlid, A. S., 1863. A List of Animals dredged near Caribou Island etc. The Canad. Natur. and Geolog. Vol. 8. 1866 (-69). Observations on the glacial Phenomena of Labrador and Maine, with a View of tho recent invertrate Fauna of Labrador. Mem. Boston Soe. Nat. Hist. Vol. I, 1860-69.

1876. Preliminary Report on a series of Dredgings... in the Gulf of Maine (1873). U. S. Comm. Fish and Fisheries P. III. Rep. of the Comm. f. 1873-7t. (Washington.)

PeArCer, F. G., 1885. Investigations on the Movements and Food of the Herring with additions to the marine Fauna of the Shetland Isles. Proc. I. Phys. Soc. Vol. 8. (Edinburgh.)

- 1902. Notes on the marine deposits of the Firth of Forth. Trans. Nat. Hist. Soc. Glasgow (N. S) Vol. 6.

Petcir, T., 1903. Marine zoology at Filey. Naturalist 1903.

Petersen, C. G., \& Levinsen, J., 1900. Travlinger i Skagerak og det nordlige Kattegat i 1897 og 1898. Beretn. f. d. Dansko Biol. Stat. 9. (Fiskeriber. f. 1898-99.) (Köbenhavn.)

Pfeffer, G., 1886. Mollusken, Krebse und Lchinodermen ron Cumberland-Sund. Jahrb. Hamburg. Wiss. Anst. 3. Jg.

- - 1890. Die Fauna der Insel Jeretik, Port Wladinir, an der Murmankiiste. I. Ibid. 7. Jg. (1889)

Puiprs, C. J., 1774. Voyage towards the North Pole 1773. Appendix. (London.)

Pocock, IR., 1889. Report of a deap-sea trawling cruise off the S. Wr. coast of Ireland. Crustacea. Ann. Mag. Nat. Hist. (6) Vol. 4.

Raskiv, W., 1907. Crustacea thoracostraca. Contr. to the Nat. Ilist. of Lambay. Irish Nat. Vol. 16. (Dublin.)

RAtibun, MARY, 1904. Decapol Crustaceans of the northwest coast of North America. IIarriman Alaska Expedition. (New York.)

- - 1905. List of the Crustacea. (Fauna of New England.) Occas. Pap. of the Boston Soc. Nat. Hist. Yol. 7.

REDEKE, H. C., 1906. Orerzicht der Uithomsten van visscherijwarnemingen met het S. S. "Wodan, 190203. Jaarb. Rijksinst. Onderzoek d. Zeo. (IIelder.)

Riciters, F., 188t. Beitrag zur Kenntnis der Crustaceenfauna des Behringsmeeres. Abh. d. Senckenb. naturf. Ges. 1884. (Frankfurt a. M.)

Römer, F., \& Scinaduiñ, F., 1900. Einleitung. Fauna aretica. Bd. 1.

Ross, J. C., 1826. ZoologJ. In: Journ. of a third royage f. the dise. of a north-west Pass. (I'arry). Appendix. 1828. Zoology. In: Narr. of an attempt to reach the North Pole 1827 und the Comm. of W. E. Parry. (Parry's fourth voy.) Appendix.

--, 1835. Marine invertebrate animals. Appendix to the Narr. of a sec. voy. in search of a north-west Pass. By Sir Jomn Ross. (London.)

RuIJs, M., 1887. Zool. Bijdr. t. d. Kennis d. Karazec. (Nederl. Pool. Exped. 1882-83.) 1. Inleiding en alge. meene mededeelingen. Bijdr. t. d. Dierk. 14. Afler. (Amsterdam.)

SAbine, E., 1824. Marine Invertebrate Animals. P'ARry's royage f. the discor. of a north-west Pass. 1819 - 20. (PArry's first royage.) Suppl. to tho Appendix.

SARs, G. O., 1869. Undersögelser orer Christianiafjordens Dybvandsfauna. Nyt Magaz. f. Naturrid. Bd. 16. (Christiania.)

1872. Undersögelser over Hardangerfjordens Fauna. I. Crustacea. Vidensk.-Selsk. Forh. f. 1871. (Christiania.)

1879. Nogle Bemærkninger om den marine Faunas Character veả Norges nordlige Krster. Tromsö Mus. Aarsh. 2.

1883. Oversigt af Norges Crustacecr, I. Tidensk. Selsk. Forhandl. f. 1882.

(1885-) 1886. Crustacea. Den norske Nordhars.Exped. (The norw. North-Atlantic Lxped.) 1876-1878. Bd. 6 (I, 1885; II [List of species], 1886). (Kristiania.)

1890. Bidrag til Kundskaben om Decapodernes Forvandlinger. III. Fam. Crangonida. Areh. f. Mathem. og Naturvid. 1890. (Kristiania.)

1899. Necount of the postembryonal development of l'andalus borealis. IRep. Norw. Fish. and Mar. Invest. Fol. I (1900). (Kristiania.)

1900. Crustacea. The Norw. North Polar Exped, 1893-1896. Sci. Res, Vol. I. (Kiristiania.)

1909. Crustacea. Rep. of the see. Norw. Aret. Exped. in the Fram 1898-1902. N:o 18. (Kristiania.)

SARS, M., 1858. Ovorsigt over do i den norsk-aretiske Region forekommende Krebsdyr. Vidensk.-Selsk. Forhandl. f. 1858 .

- - 1861. Beretning on en i Sommeren 1859 foretagen zoologisk Reise red histen af Romsdals Amt. Nyt Magaz. f. Naturvid. Bd. 11.

1861 a. Bemarkninger orer Crangonineme etc. Vidensk.-Selsk. Forh. f. 1861.

1866. Om arktiske Dyreformer i Cliristianiafjorden. Ibid. f. 1865.

Scmud, J., 1904. Fiskeriundersögelser vell Island og Feröerne i sommeren 1903. Skrifter utg. af liommiss. f. Harundersög. N:o l. (Köbenhavn.)

Scumit, J., 1904. Monographio de l'îlo Anticosti. (1'aris, Diss.)

K. Sr. Vet. Akad. Hand1. Mand 54, N:0 7, 
Scort, Tri, 1888. A revised list of the Crinstacea of the Wirth of Forth. 6. Amn. liep. of the Fish. Board $\mathrm{f}$. Scot. land. P. 3. (zitiert nach st.tPPERs). (Edinburuh.)

1897. The marino Fishes and Invertebrates of Loch Fye. Ibid. 15. Rep. 1'. 3.

$18: 7$ a. lieport on a collection of Marine I) rederings... made on the West Coast of Scotland. J'roc. R. Phys. Soc. lidinburgh. Yol. 13 (1894-97).

1899. Report on the Marine and Freshater Crustacea from Franz Josef Land, coll. ly Mr. Bruce. Journ. Liun. Soc, Zool. Yol. 27. (London.)

Surru, S. I., 1879. 'The stalkeyed Crustaceans af the Atlantic Coast of North Anerica north of Cape Cod. 'Trans. Comnect. Acael. Arts Ści. Vol. 5.

, 1880. Notes sur les Crustaces recueillis... aux lles de Vancouver et de la lieine-Charlotte. Commiss. géol. du Canada, liapp. d. opér. do 1878-79. (Anch in: Rep). Geol. and Nat. Ilist. Surr. of Canada 1878-79.)

- 1882. Report on the Crustacea. (Rep. on the Res. of Dredg. und. the Superv. of 1. Agassiz, on the Last Coast of the United States 1880, by the ... Blake».) Bull. II Ls. Comp. Zool. Vol. 10. (Cambridge, Jass.) 1884. List of the Crustacea dredged on the coast of Labrador hy the Exped.... of W. A. Stearns, in 1882. I'roc. U. S. Nat. M[us. Vol. 6 (f. 1883). (Washington.)

$188+$ a. Review of the Marine Crustacea of Labrador. Ibid.

- $188+\mathrm{h}, 1887$. Report on the Decapod Crustacea of the Albatross Drerlgings off the cast const of the Lnited states in 1883. U. S. Comm. of F'ish and Fisher., Rep. of the Commiss. f. $1882,1885$.

SuItI, S. I., \& IIALGer, 0., 1874. Report on the Dredgings in the Region of St. Georges Banks, in 1872. Trans. Connect. Acat. Arts Sei. Vol. 3.

Sowenzy, J., 1806. The British Miscellany. (London.)

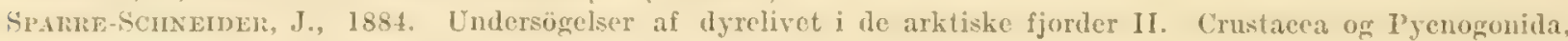
indsamlede i Kivanangsforden 1881. 'l'romsö Mus. Aarsh. 7.

STAPERS, L., 1911. Crustacés Malacostracés. Due d'Orléans, Camp. aret. do 1907. (Bruxelles.)

Stembixg, Tri, 1900. Aretic Crustacea: Bruce Collection. Ann. Mag. Nat. Ilist. (7) Vol. 5.

STHPIExSEx, K., 1910. Revideret lortegnelse over Danmarks marine Arter af Decapoda. Vid. Meldel. Naturh. Foren. Köbenhavn. Bal. 61 f. 1909.

1910 a. Skjoldkrebs (Storkrebs 1.). Danmarks Fauma. (Köbenharn.)

--, 1912. Report on the Malacostraca, Pyenogonida and some Entomostraca, coll. by the Danmark Exped. to North-east Greculand. (Danmark-liksp. 1906-1908. Bd.5, X:o 11). Meddel. om Grönl. 45. (Köben. harn.)

_- 1912 a. Report on the Malacostraca coll. by the "Tjalfe»-Expedition. Vid. Meddel. Naturh. Foren. Köben. linvn. Bd. 64 .

1913. Grönlands Krebsdyr og Pycnogonider (Conspectus (Crustaceorum et l'yenogonielorum Groenlandix). Meddel. cm Grönl. 13d. 22. (Köhenhavn.)

1913 a. Aceount of the Crustacea and Pyenogonida, collected by Dr. V. Nordmann 1911 in West Greenland. Ibid. Bd. 51. - In den Ühersichten der geographisehen Verbreitung weist osterIexsex 1913, sowohl auf diese wie auf die folgende Arheit hin; durch cin Verschen - weil dic letztere in die Literaturverzeichnis der ersteren aufgenommen ist - wurde die Arheit 191:3 a nieht hesonders erwihnt.

Strkm, A., 1895. Die Insel Solowetzk im Weissen Meero ete. Sitzungsh. Naturf. Gez. Dorpat. Bd. 10.

strmpsox, W., 185t. Synopsis of the marine Invertebrata of Gr. Manan. Smiths. Contrib. Pol. 6. (Washington.)

-, 1859-60. Notes on North American Crustacea. I, II. Ann. Lye. Nat. Hist. New York. Tol. 7. 1860. l'rodromus descriptionis animalium evertebratorum, quao in Expedit. ad Ocean. Jacif. ecpt... observavit et descripsit WV. Stimpson. Proc. Acad. Nat. Scienc. Philadelphia 1860.

-, 1863. Synopsis of the Marine Invertebrata collected by the late Aretic Expedition, under Dr. I. J. Hares. Thicl. 1863.

1871. Notes on North American Crustacen, in the Museum of the Smithson. Inst. N:0 3. Ann. Ixc. Nat. IIist. of New lork. Vol. 10 (1874).

1907. Report on the Crustacea (Brachyura and Anomura), coll. by the North Pacific Explor. Exped. 18531856. (Herausg. von M. Rathhun). Smiths. Instit. Washington, Misc. Collect. Vol. 49.

STom, Y., 1878. Jeretning om Selskabets zoologisko Samling i Aaret 1877. K. Norske Vidensk.-Selsk. Skrifter. Bd. 8 (187t-77). (Trondhjem.)

1880, 1881. Bidrag til Kundskab on Trondhjemsfjortens Fauna. II, III. Ibid. 1879, 1880.

1901. Oversigt orer 'Tronthjemsfjordens fauna. Trondhjems biol. Stat. Medd. Stationsanl. arbeidskommité 1901. ('Trykt som M. S.) (Trondhjem.)

Stuxberg, A., 188\%. Evertebratfaunan i Sibiriens ishaf. Tega-Exped. retensk. iakttag. Bd. 1. (Stockholm.)

- 1886. Faunan pai och kring Noraja Semlja. Ibid. Bd. 5.

Scenstia Hydrografisk-73iologisha hommissionen, 1910. Iydrographisehe Beolachtungen der sehwed. Exped. n. Spitzbergen 1908. (Zool. Erg. d. Exp. 1903., 'T. I.) K. Sv. Vet.-Akad. IIandI. Bd. 45. (Stocliholm.)

'Tríz, HJ., 1907. Om utvecklingen af Sveriges zoologiska hafsstation Kristineberg och on djurlifret i angransande haf och fjordar. Is. Srenska Tet.-Akad., Ark. f. Zool. Bd. 4 (Stockholm.) 
Trionpsox, W., 1842. The Crustacea of Irelaud. Ann. Mag. Nat. Hist. Fol. 10.

, 1844. Report on the Fama of Ireland. Rep. Brit. Ass. f. 1813.

, 1856. The natural History of Ireland. Vol. IV. (London.)

Tryos, F., 1903. Iakttagelser om fisk och decapoder m. m. frin skagerak och Katteratt 1901-02. Srenska Hydr. Biol. Kommiss. Skrifter I.

VAniörfex, E., 1897. Die Fauna und Flora Grönlands. Grönl.-Exped. d. Ges. f. Erdk. z. Berlin 1891-1893. 13d. II. 1 .

Vereile, A. E., 1873, 1874. Results of recent Dredging Expeditions on the Coast of New England. Amer. Journ. Sei. Arts. (3) Vol. $5-7$.

_- \& Ratnbun, R., 1880. List of Marine Invertebrata from the New Lngland coast. I'roc. U. S. Nat. Mus. Vol. 2 (1879). (Washington.)

Wagner, N., 1885. Die Wirlsellosen des Weissen Meeres. (Leipzig.)

WALKer, A. 0., 1886, 1889, 1892. Fanna of Liverpool Bay. 1, 2, 3. Rep. (Rep. of Podophth, u. a.). 1886 anch in:- Proc. Lit. Phil. Soc. Liverp. Vol. 40, App.; 1889, 1892 anch in: Proc. 'Trans. Liverp. Biol, Soc. Tol. 3.

WaLker, D., 1860. Notes on the aretic Zoology of the last aretic Jixpedition. Journ. 1R. Dublin Soc. Vol. 3.

Winteares, J. F., 187. Notes on a Deep-sea Dredging-Expedition round the Island of Anticosti in the Gulf of St. Lawrence, Am. NIag. Nat. Hist. (4) Vol. 10.

187t. On recent Deep-Sea Dredg. Oper, in the Gulf of St. Lawrence. Amer. Joum. Sei. Arts. (3) Vol. 7. 1901. Catalogue of Marino Invertchrata of castern Canada. Gcol. Surr. Canada. (Ottawa.)

Wolleber, 1., 1900. Decapoda collected during the fishing Investigations dir. by Dr. Iljort in 1897-98. Lep. on Norw. Fish. and Marine Invest. Vol. 1. (Kristiania.)

1908. Remarks on Decapod Crustaceans of the North Atlantic and the Norwegian Fjords. Bergens Mus. Aarb. 1908. 


\section{Inhalt.}

Vormurt

\section{Spezieller Teil:}

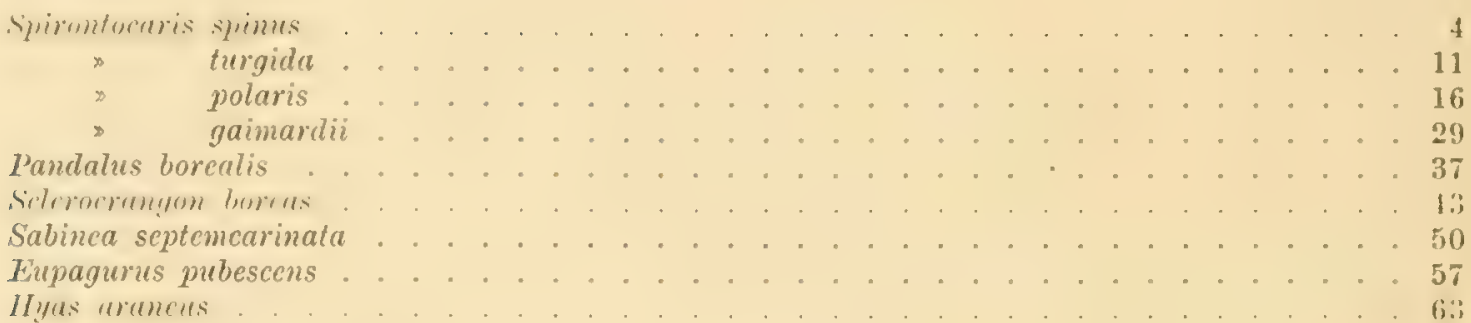

\section{Allemeiner 'Teil:}

Die Verbreitung der Decapoden im Eisfjord.

Artanzahl und Häufigkeit . . . . . . . . . . . . . . . . . . . . . . . . 68

Einwirliung der Bodenbeschaffenheit . . . . . . . . . 68

Einwirkung der Tiefe: Bathymetrische Verbreitung . . . . . . . . . . . . . . . . 70

Einwirkung der Wassertemperatur . . . . . . . . . . . . . . . . . 71

Übrige Falitoren ..... . 73

Zusammenwirken der Falktoren Bodenbeschaffenheit, Tiefe und Wassertemperatur: das Terbreitungsbild Are Derapoten im Fisfiont

Allgemeine Verbreitung ler Jecapoden der arkischen Region.

Ilistorischer Überblick . . . . . . . . . . . . . . . . . . 76

Die nord-sidliche Vorbreitung der Nordmeernrten . . . . . . . . . . . . . .

Arktische Arten

Hocharlische Arten . . . . . . . . . . . . . . . . . . . . . . . . .

Panarktische Arten . . . . . . . . . . . . . . . . . . . . . . . . . . . 80

Arktisch-boreale Arten . . . 82

Panarktisch-boreale Arten. Herkunft derselben . . . . . . . . . . . . . . . . . . 82

Niederarktisch boreale Arten. Herkunft derselben . . . . . . . . . . . . . . . 84

Boreale Gäste in der arktischen legion . . . . . . . . . . . . . . . . . . . 89

Zusammenfassende Übersicht der nord-südlichen Verbreitung . . . . . . . . . . . . . . . 90

bie ost-westlicho Verbreitung . . . . . . . . . . . . . . . . . . . . 11

Zirkumpolare Arten . . . . . . . . . . . . . . . . . . . . . . . . . .

Atlantische Arten . . . . . . . . . . . . . . . . . . . . . 92

Westliche Arten . - 93

Pazifische Arten . . . . . . . . . . . . . . . . . . . . . 94

Die Ursachen der verschiedenen Verbreitung . . . . . . . . . . . . . . . . . 94

Jie Verbreitung der Jecapoden im Spitzbergengebiet . . . . . . . . . . . . . . 97

Anmerkungen zu den Fundortsverzcichnissen .................... J00

Literaturverzeichnis . . . . . . . . . . . . . . 101

Tryckt den 9 december 1916 

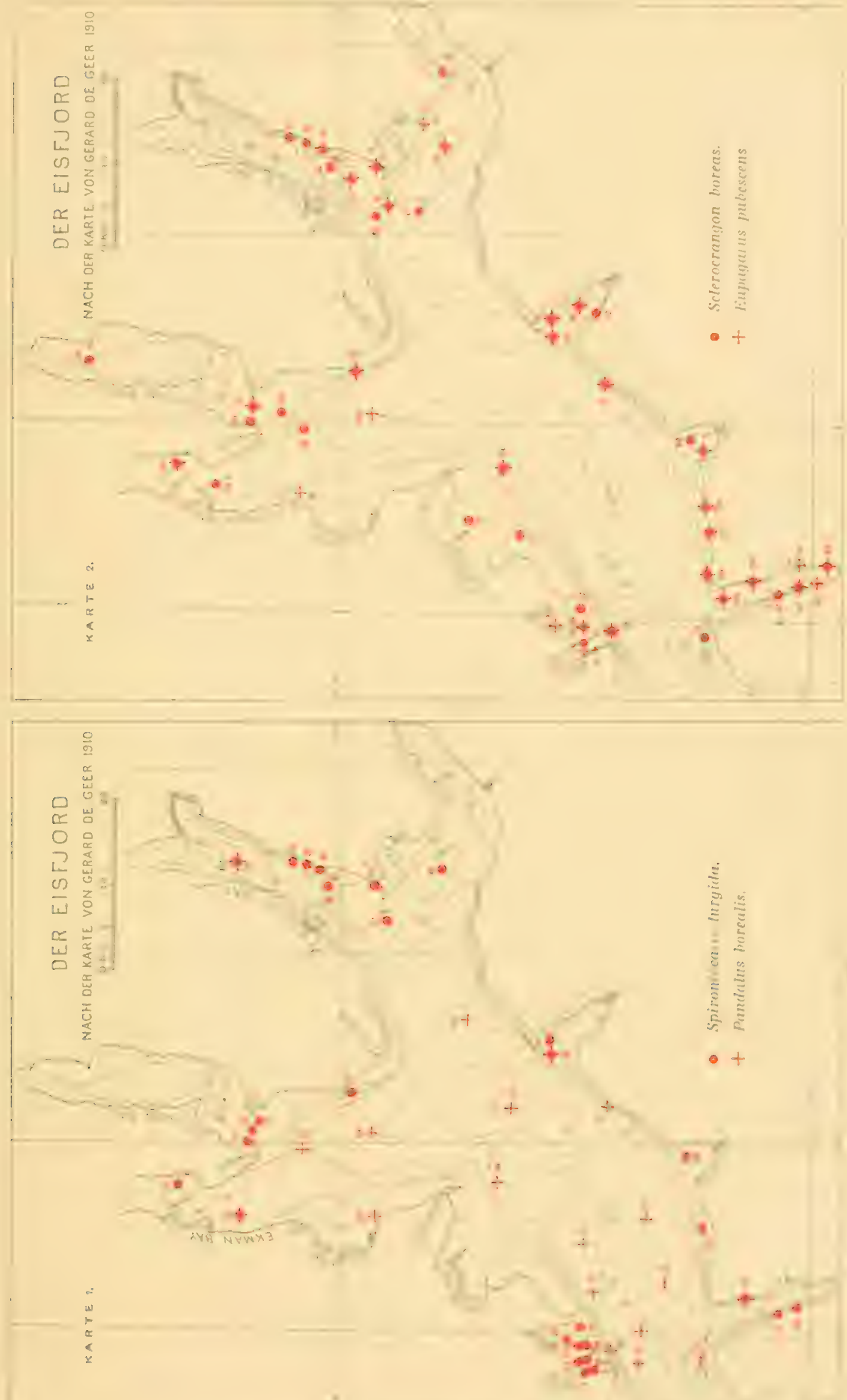


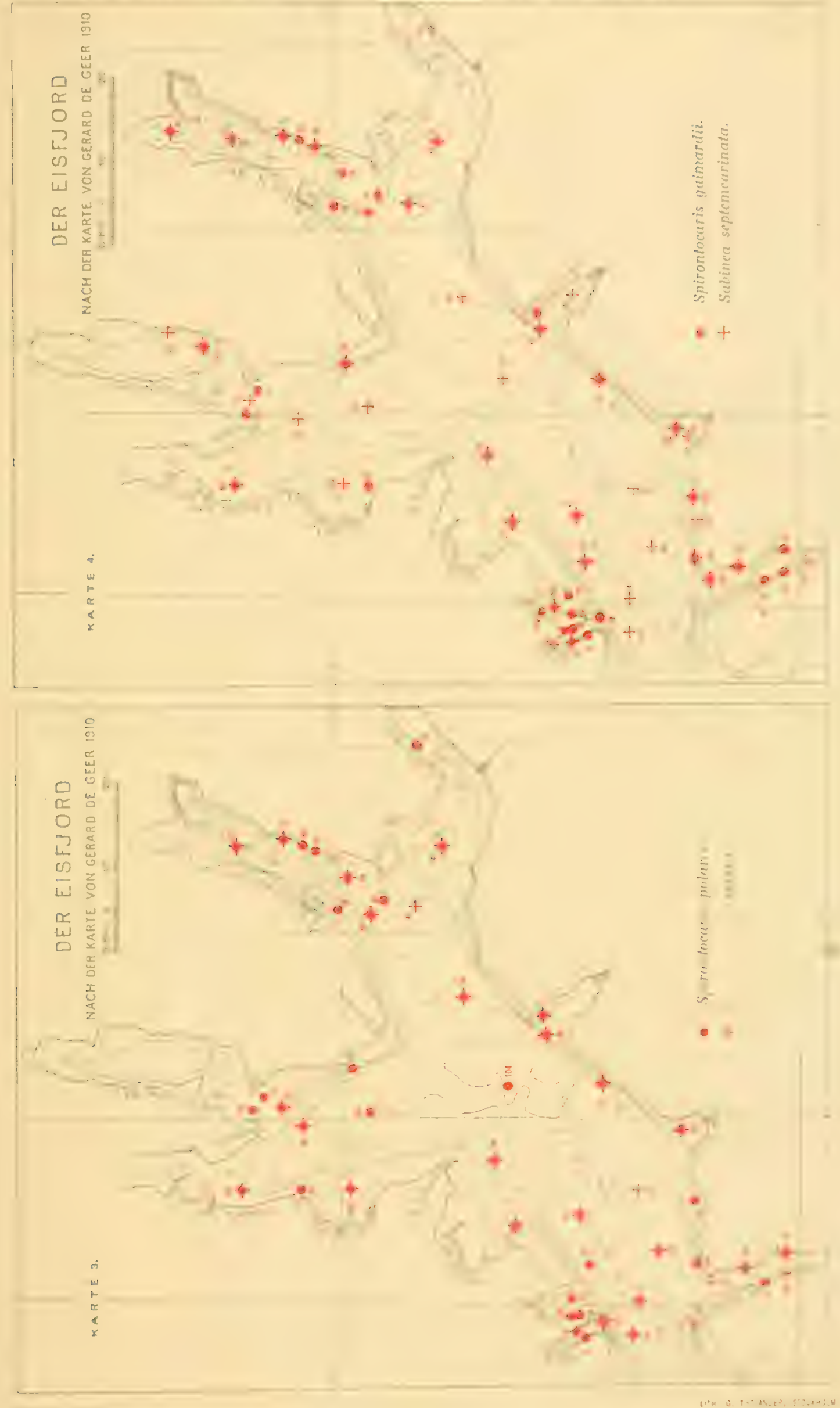


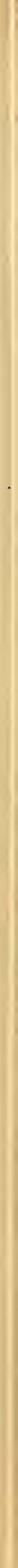





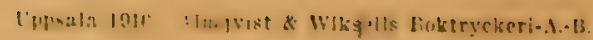





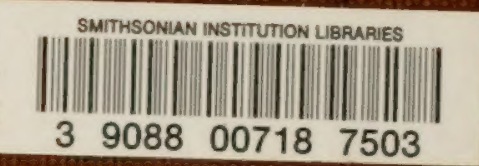

